LA-13603-MS

Approved for public release: distribution is unlimited.
Background Radioactivity in River and

Reservoir Sediments near Los Alamos, New Mexico

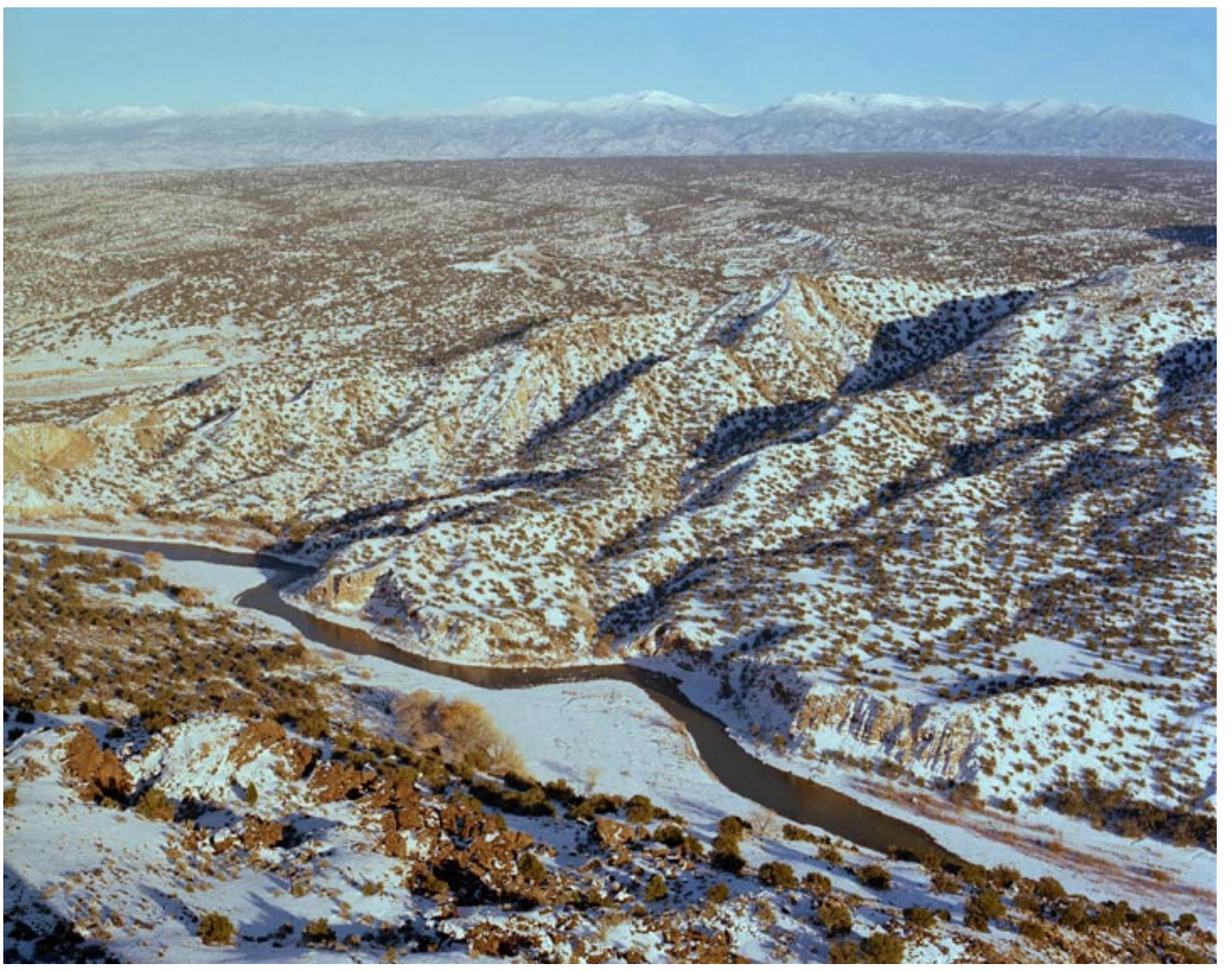

\section{Los Alamos}

NATIONAL LABORATORY

Los Alamos National Laboratory is operated by the University of California for the United States Department of Energy under contract W-7405-ENG-36. 


\section{Edited by Martha Lee DeLanoy and Faith Harp, Group IM-1 Photocomposition by Kathy E. Valdez, Group IM-1}

Cover: Snow-covered view of the Rio Grande looking east toward the Sangre de Cristo Mountains from Overlook Park in White Rock, New Mexico.

An Affirmative Action/Equal Opportunity Employer

This report was prepared as an account of work sponsored by an agency of the United States Government. Neither The Regents of the University of California, the United States Government nor any agency thereof, nor any of their employees, makes any warranty, express or implied, or assumes any legal liability or responsibility for the accuracy, completeness, or usefulness of any information, apparatus, product, or process disclosed, or represents that its use would not infringe privately owned rights. Reference herein to any specific commercial product, process, or service by trade name, trademark, manufacturer, or otherwise, does not necessarily constitute or imply its endorsement, recommendation, or favoring by The Regents of the University of California, the United States Government, or any agency thereof. The views and opinions of authors expressed herein do not necessarily state or reflect those of The Regents of the University of California, the United States Government, or any agency thereof. Los Alamos National Laboratory strongly supports academic freedom and a researcher's right to publish; as an institution, however, the Laboratory does not endorse the viewpoint of a publication or guarantee its technical correctness. 
Background Radioactivity in River and

Reservoir Sediments near Los Alamos, New Mexico

Stephen G. McLin

Dale W. Lyons 



\section{CONTENTS}

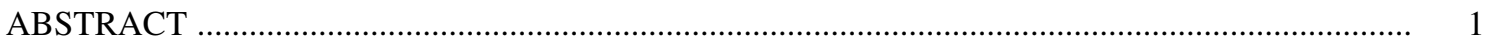

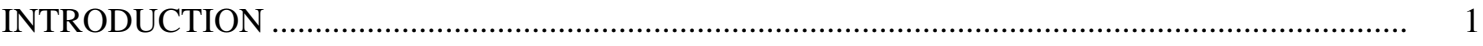

SAMPLE COLLECTION PROCEDURES ………................................................................. 5

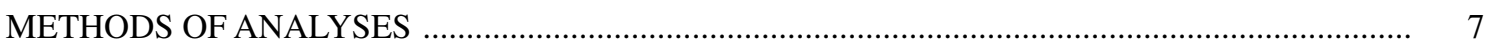

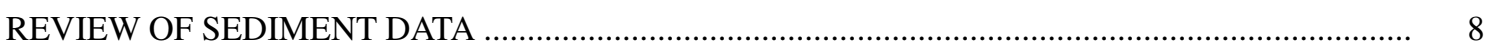

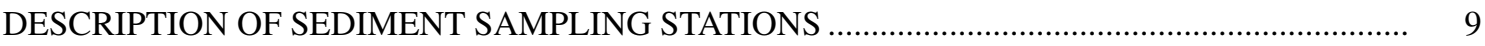

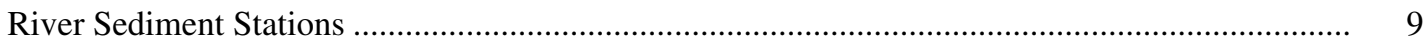

Reservoir Sediment Stations ............................................................................................... 11

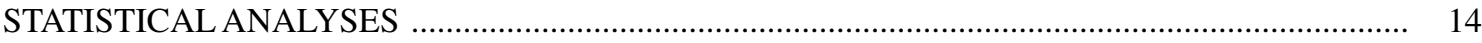

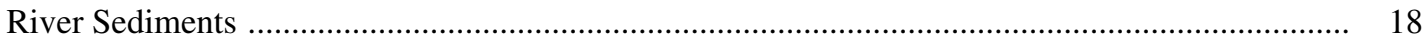

Reservoir Sediments ......................................................................................................... 19

Comparison of River and Reservoir Sediments .................................................................... 19

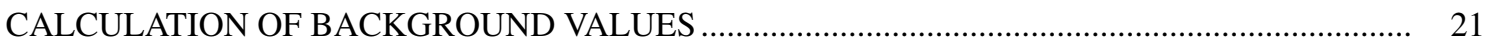

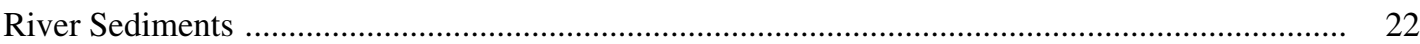

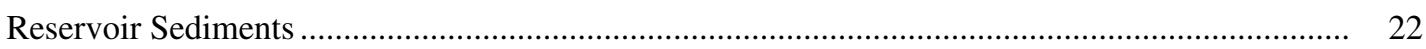

Comparisons of Background Values with Other Published Results .............................................. 22

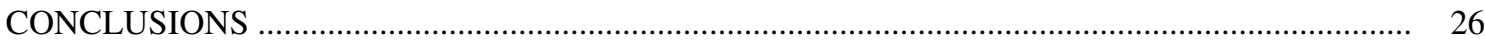

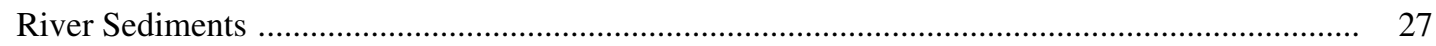

Reservoir Sediments ......................................................................................................... 28

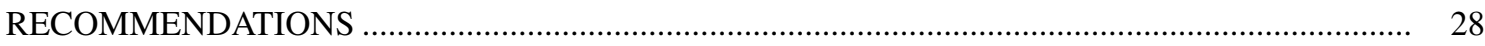

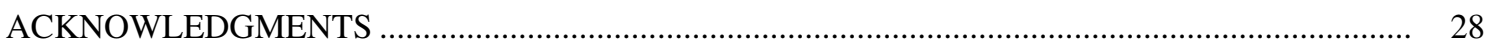

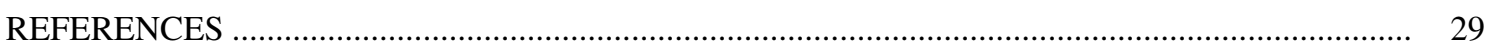

APPENDIX A.

RADIOCHEMICAL ANALYSES FOR RIVER SEDIMENTS _................................................. 33

APPENDIX B.

RADIOCHEMICAL ANALYSES FOR RESERVOIR SEDIMENTS ........................................... 47

APPENDIX C.

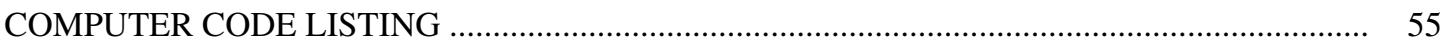

APPENDIX D.

PROBABILITY PLOTS FOR GROUP I RIVER SEDIMENTS …….......................................... 57

APPENDIX E.

PROBABILITY PLOTS FOR GROUP I RESERVOIR SEDIMENTS …………........................... 79 


\section{FIGURES}

1. General drainage basin map of the northern Rio Grande. ..................................................... 2

2. Sediment sampling locations in the vicinity of Los Alamos National Laboratory. ....................... 3

D-1. Probability plot for tritium in Group I river sediments............................................................. 58

D-2. Normalized plots for tritium in Group I river sediments. .................................................... 59

D-3. Probability plot for strontium-90 in Group I river sediments. ..................................................... 60

D-4. Normalized plots for strontium-90 in Group I river sediments. ................................................ 61

D-5. Probability plot for cesium-137 in Group I river sediments. .................................................... 62

D-6. Normalized plots for cesium-137 in Group I river sediments. ................................................. 63

D-7. Probability plot for total uranium in Group I river sediments. .................................................... 64

D-8. Normalized plots for total uranium in Group I river sediments. ................................................ 65

D-9. Probability plot for plutonium-238 in Group I river sediments. ................................................ 66

D-10. Normalized plots for plutonium-238 in Group I river sediments. ............................................... 67

D-11. Probability plot for plutonium-239,-240 in Group I river sediments. .......................................... 68

D-12. Normalized plots for plutonium-239,-240 in Group I river sediments. ....................................... 69

D-13. Probability plot for americium-241 in Group I river sediments. ................................................ 70

D-14. Normalized plots for americium-241 in Group I river sediments. .............................................. 71

D-15. Probability plot for gross alpha in Group I river sediments. ..................................................... 72

D-16. Normalized plots for gross alpha in Group I river sediments. .................................................. 73

D-17. Probability plot for gross beta in Group I river sediments. ......................................................... 74

D-18. Normalized plots for gross beta in Group I river sediments. ....................................................... 75

D-19. Probability plot for gross gamma in Group I river sediments. .................................................. 76

D-20. Normalized plots for gross gamma in Group I river sediments................................................ 77

E-1. Probability plot for tritium in Group I reservoir sediments. .................................................... 80

E-2. Normalized plots for tritium in Group I reservoir sediments. .................................................. 81

E-3. Probability plot for strontium-90 in Group I reservoir sediments. ............................................ 82

E-4. Normalized plots for strontium-90 in Group I reservoir sediments. ........................................ 83

E-5. Probability plot for cesium-137 in Group I reservoir sediments. ................................................. 84

E-6. Normalized plots for cesium-137 in Group I reservoir sediments. .............................................. 85

E-7. Probability plot for total uranium in Group I reservoir sediments. ......................................... 86

E-8. Normalized plots for total uranium in Group I reservoir sediments. ........................................... 87

E-9. Probability plot for plutonium-238 in Group I reservoir sediments. ............................................ 88

E-10. Normalized plots for plutonium-238 in Group I reservoir sediments. ........................................ 89

E-11. Probability plot for plutonium-239,-240 in Group I reservoir sediments. .................................. 90

E-12. Normalized plots for plutonium-239,-240 in Group I reservoir sediments. ................................. 91

E-13. Probability plot for americium-241 in Group I reservoir sediments. ......................................... 92

E-14. Normalized plots for americium-241 in Group I reservoir sediments. ...................................... 93

E-15. Probability plot for gross alpha in Group I reservoir sediments. ................................................ 94 
E-16. Normalized plots for gross alpha in Group I reservoir sediments.

E-17. Probability plot for gross beta in Group I reservoir sediments.

E-18. Normalized plots for gross beta in Group I reservoir sediments. ....................................... 97

E-19. Probability plot for gross gamma in Group I reservoir sediments..................................... 98

E-20. Normalized plots for gross gamma in Group I reservoir sediments. ...................................... 99

\section{TABLES}

1. Analytical Laboratory Limits of Detection for Individual Analytes.

Established SAL Values Are Shown for Comparison.......................................................

2. Criteria Used for Data Elimination. ................................................................................. 8

3. Total Number of Sediment Samples Reviewed by ESH-18. ...................................... 10

4. Mean Daily Discharge at Selected USGS Gages. See Web Site http://h2o.usgs.gov. ............... 12

5. Summary of Important Reservoir Sedimentation Characteristics. See National Inventory of Dams Database and Web Site for Additional Information (http://npdp.stanford.edu).

6. Computed Lilliefors Statistic to Test for Normal, Lognormal, and Square-Root Distributions at Individual Sampling Stations.

7. Computed Lilliefors Statistic to Test for Normal, Lognormal, and Square-Root Distributions at Grouped Sampling Stations.

8. Statistical Comparison of Group I River and Group 1 Reservoir Sediments.

9. Statistical Summary for Group I River Sediment Background Stations. The Sampling Period Runs from 1974 through 1997.

10. Statistical Summary from Group I Reservoir Sediment Background Stations. The Sampling Period Runs from 1979 through 1997....................................................... 24

11. Comparison of Reported Background Radioactivity in Sediments...................................... 25

12. Recommended Upper Limit of Background (BGUL) Radioactivity in Sediments near Los Alamos.

A-1. Sediment Station: Rio Chama at Chamita .................................................................... 34

A-2. Statistical Summary for Rio Chama at Chamita .................................................................. 34

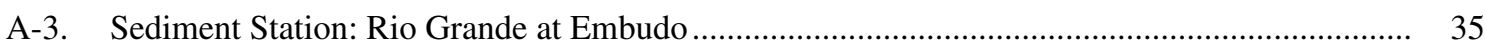

A-4. Statistical Summary for Rio Grande at Embudo ........................................................

A-5. Sediment Station: Rio Grande at Otowi Bridge ........................................................... 36

A-6. Statistical Summary for Rio Grande at Otowi Bridge ....................................................... 36

A-7. Sediment Station: Rio Grande below Sandia Canyon ..................................................... 37

A-8. Statistical Summary for Rio Grande below Sandia Canyon ............................................ 37

A-9. Sediment Station: Rio Grande below Pajarito Canyon ........................................................ 38

A-10. Statistical Summary for Rio Grande below Pajarito Canyon .............................................. 38

A-11. Sediment Station: Rio Grande below Ancho Canyon ..................................................... 39

A-12. Statistical Summary for Rio Grande below Ancho Canyon ................................................. 39

A-13. Sediment Station: Rio Grande below Frijoles Canyon ........................................................

A-14. Statistical Summary for Rio Grande below Frijoles Canyon .............................................. 40 
A-15. Sediment Station: Rio Grande below Cochiti Reservoir Spillway .....

A-16. Statistical Summary for Rio Grande below Cochiti Reservoir Spillway

A-17. Sediment Station: Rio Grande at Bernalillo

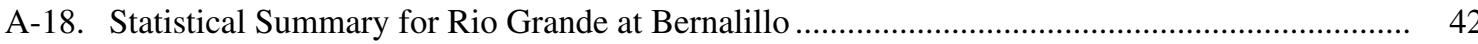

A-19. Sediment Station: Jemez River near Jemez Pueblo .............................................................

A-20. Statistical Summary for Jemez River near Jemez Pueblo ......................................................

A-21. Summary Statistics: All Regional River Sediment Stations (Combined Group I and Group II Stations)

A-22. Summary Statistics: Group I River Sediment Stations (Chamita, Embudo, Cochiti Spillway, Bernalillo, and Jemez)

A-23. Summary Statistics: Group II River Sediment Stations (Otowi, Sandia, Pajarito, Ancho, and Frijoles)

A-24. Summary Statistics: Group I vs. Group II at 95\% Significance Level - Student's t-Test with Independent Samples and Unequal Variance

A-25. Summary Statistics: Group I vs. Group II at 95\% Significance Level - Student's t-Test with Independent Samples and Equal Variance

A-26. Summary Statistics: Group I vs. Group II at 95\% Significance Level Kruskal-Wallis Test with Independent Samples

B-1. Rio Chama Drainage Basin: Abiquiu Reservoir ........................................................

B-2. Statistical Summary for Abiquiu Reservoir ..................................................................

B-3. Rio Chama Drainage Basin: El Vado Reservoir ..............................................................

B-4. Statistical Summary for El Vado Reservoir ............................................................ 49

B-5. Rio Chama Drainage Basin: Heron Reservoir ..........................................................

B-6. Statistical Summary for Heron Reservoir ............................................................... 50

B-7. Rio Grande Drainage Basin: Cochiti Reservoir .............................................................. 51

B-8. Statistical Summary for Cochiti Reservoir ................................................................ 52

B-9. Rio Grande Drainage Basin: Rio Grande Reservoir ...................................................... 52

B-10. Statistical Summary for Rio Grande Reservoir ............................................................ 52

B-11. Summary Statistics: All Reservoir Sediment Stations (Abiquiu, El Vado, Heron, Cochiti, and Rio Grande) ................................................... 53

B-12. Summary Statistics: Group I Reservoir Sediment Stations (Abiquiu, El Vado, Heron, and Rio Grande) ....................................................................... 53

B-13. Summary Statistics: Group II Reservoir Sediment Stations (Cochiti) ................................. 53

B-14. Summary Statistics: Group I vs. Group II at 95\% Significance Level - Student's t-Test with Independent Samples and Unequal Variance ........... 54

B-15. Summary Statistics: Group I vs. Group II at 95\% Significance Level - Student's t-Test with Independent Samples and Equal Variance .............. 54

B-16. Summary Statistics: Group I vs. Group II at $95 \%$ Significance Level - Kruskal-Wallis Test with Independent Samples 


\title{
BACKGROUND RADIOACTIVITY IN RIVER AND RESERVOIR SEDIMENTS NEAR LOS ALAMOS, NEW MEXICO
}

by

\author{
Stephen G. McLin and Dale W. Lyons
}

\begin{abstract}
As part of its continuing Environmental Surveillance Program, regional river and lake-bottom sediments have been collected annually by Los Alamos National Laboratory (the Laboratory) since 1974 and 1979, respectively. These background samples are collected from three drainage basins at ten different river stations and five reservoirs located throughout northern New Mexico and southern Colorado. Radiochemical analyses for these sediments include tritium, strontium-90, cesium-137, total uranium, plutonium-238, plutonium-239,-240, americium-241, gross alpha, gross beta, and gross gamma radioactivity. Detection-limit radioactivity originates as worldwide fallout from aboveground nuclear weapons testing and satellite reentry into Earth's atmosphere. Spatial and temporal variations in individual analyte levels originate from atmospheric point-source introductions and natural rate differences in airborne deposition and soil erosion. Background radioactivity values on sediments reflect this variability, and grouped river and reservoir sediment samples show a range of statistical distributions that appear to be analyte dependent. Traditionally, both river and reservoir analyte data were blended together to establish background levels. In this report, however, we group background sediment data according to two criteria. These include sediment source (either river or reservoir sediments) and station location relative to the Laboratory (either upstream or downstream). These grouped data are statistically evaluated through 1997 , and background radioactivity values are established for individual analytes in upstream river and reservoir sediments. This information may be used to establish the existence and areal extent of trace-level environmental contamination resulting from historical Laboratory research activities since the early 1940s.
\end{abstract}

\section{INTRODUCTION}

Sediment transport associated with surface water runoff is a significant mechanism for contaminant migration in the environment. Contaminants originating from airborne deposition, effluent discharges, or unplanned releases can become attached to soils or sediments by absorption, adsorption, or ion exchange. As part of its ongoing Environmental Surveillance Program, Los Alamos National Laboratory (the Laboratory) has been monitoring radiochemicals in river and reservoir sediments since 1974 and 1979, respectively. River sediments are collected annually from stream channels within and adjacent to the Laboratory, including those with either perennial or ephemeral flows. Reservoir sediments are also collected annually from regional lakes located upstream and downstream of Los Alamos. An essential part of this surveillance program is the establishment of background radioactivity values for important radiochemicals. 
In this report, estimates for background levels are refined from previous studies for strontium-90, cesium-137, total uranium, plutonium-238, and plutonium-239,-240. In addition, new background values are established for tritium, americium-241, gross alpha, gross beta, and gross gamma radioactivity in sediments.

Ten background locations for river samples are included in the environmental surveillance network. In addition, five regional reservoirs serve as background monitoring stations. Locations of these background sediment-sampling stations are shown in Figs. 1 and 2. Traditionally, individual analyte values from all of these background stations have been blended together, and composite background values for sediments were established for each analyte. In this report, however, we separate sediments according to a river or reservoir source. In addition, we also separate individual sampling stations into upstream and downstream locations relative to the Laboratory. These groupings result in five upstream and five downstream river stations, and four upstream and one downstream reservoir stations. These grouping are further described below. Grouped statistical analyses are made for individual analytes to establish potential differences in grouped mean radionuclide values. Then upper limits for background radionuclide levels in river and reservoir sediments are established for individual analytes. Finally, as a convenience to the reader, comparisons to other background levels for sediment data collected at different locations are made.

Background radioactivity values in sediments are often below conventional analytical detection limits. Analytical uncertainties for these samples often exceed reported radioactivity values. These sediment samples contain trace amounts of radioactivity from worldwide fallout associated with aboveground nuclear weapons testing, satellite reentry and burn-up in the earth's atmosphere, and erosion from natural sources (e.g., uranium). Here trace amounts of radioactivity are defined to be less than or slightly above detection limits for individual isotopes. Typical detection limits and computed screening action levels (SALs) are listed in Table 1. SAL values are screening levels below a level of human health risk; they are discussed

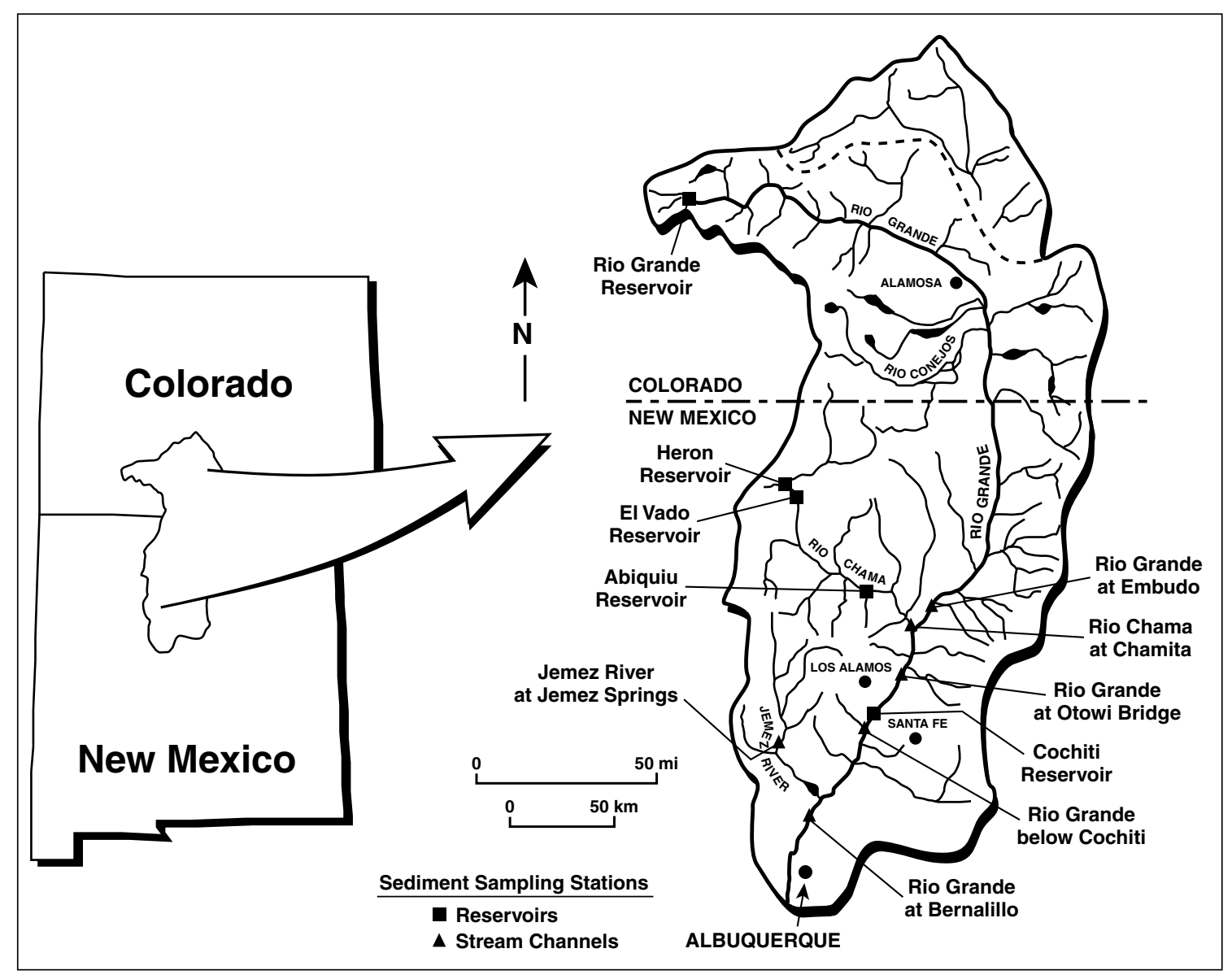

Fig. 1. General drainage basin map of the northern Rio Grande. 


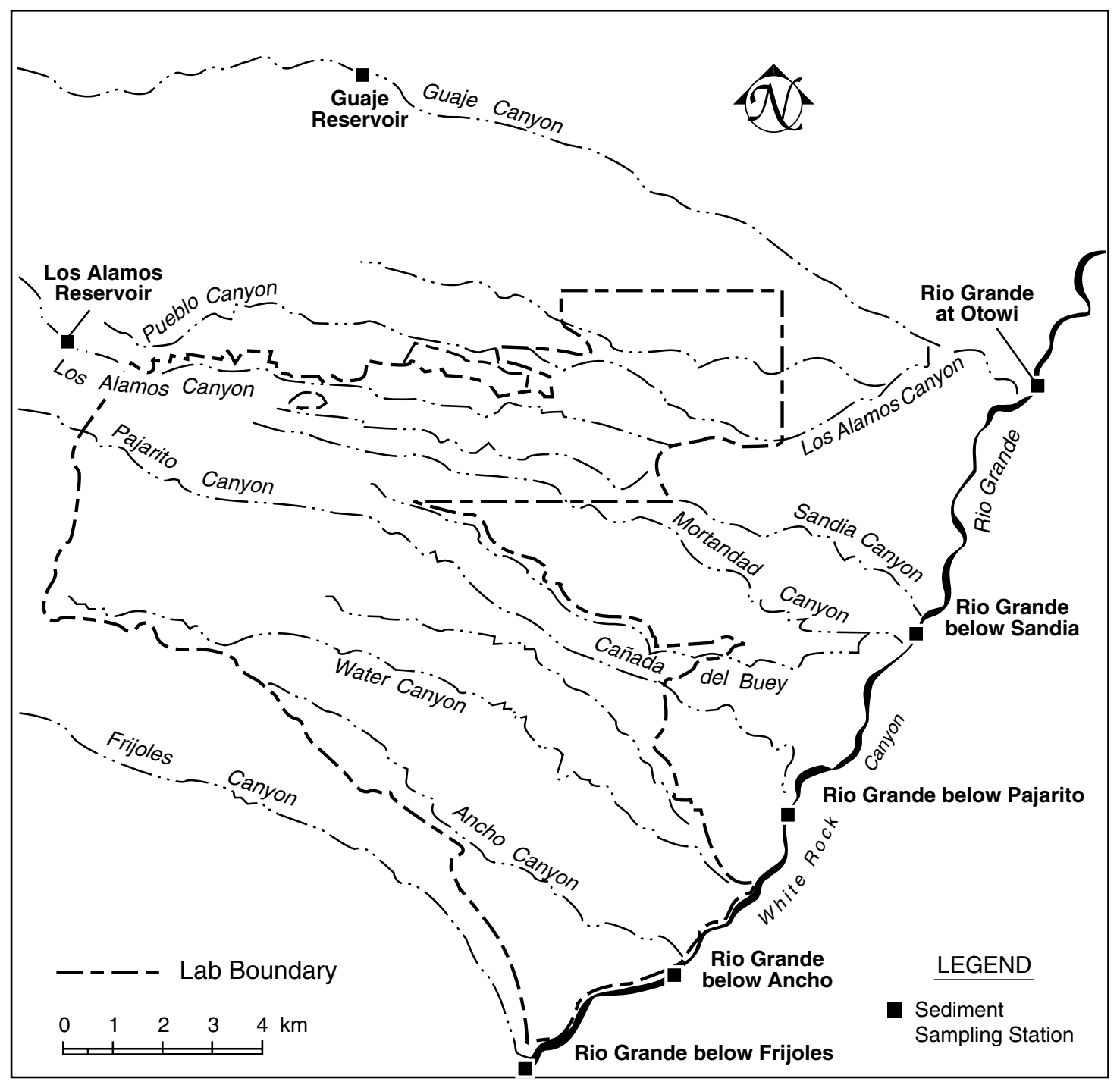

Fig. 2. Sediment sampling locations in the vicinity of Los Alamos National Laboratory.

below. The statistical distribution, range, and upper limit for background radioactivity in these sediments (i.e., combined fallout and natural sources) provide an essential base line for comparison of regional and on-site sediment data that have been potentially impacted by Laboratory operations.

There are no federal or state regulatory standards for contaminants on soils or sediments that can be used for direct comparisons with surveillance data. Instead, contaminant amounts in sediments may be interpreted in terms of toxicity or radiological dose to humans, assuming the contaminated particles are either ingested or inhaled. The data can also be compared with radioactivity values attributable to worldwide fallout or natural background radioactivity established here. SALs are also used by the Laboratory's Environmental Restoration (ER) Project to identify contaminants at levels of concern. SAL values are derived from toxicity values and exposure parameters using data from the US Environmental Protection Agency (US EPA, 1994). Finally, the total effective dose equivalent (TEDE) to Los Alamos area residents is listed in the annual environmental surveillance reports for important radiochemical values examined in this report. (Environmental Assessments and Resource Evaluation Group, 1996; Environmental Protection Group, 1990 and 1992-1995; Environmental Studies Group, 1975-1977; Environmental Surveillance and Compliance Programs, 1997; Environmental Surveillance Group, 1978-1989; Environmental Surveillance Program, 1996 and 1998). 
Table 1. Analytical Laboratory Limits of Detection for Individual Analytes. Established SAL Values Are Shown for Comparison.

\begin{tabular}{|c|c|c|c|c|}
\hline Parameter & $\begin{array}{c}\text { River } \\
\text { Sediments }^{\text {a }}\end{array}$ & $\begin{array}{c}\text { Reservoir } \\
\text { Sediments }^{\text {a }}\end{array}$ & $\begin{array}{c}\text { Reservoir } \\
\text { Sediments }^{\text {a }}\end{array}$ & $\mathbf{S A L}^{\mathbf{b}}$ \\
\hline Laboratory Sample Size (1974-97) & $10 \mathrm{~g}$ & --- & --- & --- \\
\hline Laboratory Sample Size (1979-97) & --- & $10 \mathrm{~g}$ & $1,000 \mathrm{~g}$ & --- \\
\hline Tritium & $0.7 \mathrm{nCi} / 1$ & $0.7 \mathrm{nCi} / 1$ & --- & $20 \mathrm{nCi} / 1$ \\
\hline${ }^{90} \mathrm{Sr}(1974-96)^{\mathrm{c}}$ & $0.05 \mathrm{pCi} / \mathrm{g}$ & $0.05 \mathrm{pCi} / \mathrm{g}$ & --- & --- \\
\hline${ }^{90} \mathrm{Sr}(1997)^{\mathrm{c}}$ & $1.00 \mathrm{pCi} / \mathrm{g}$ & $1.00 \mathrm{pCi} / \mathrm{g}$ & --- & $5.7 \mathrm{pCi} / \mathrm{g}$ \\
\hline${ }^{137} \mathrm{Cs}$ & $0.05 \mathrm{pCi} / \mathrm{g}$ & $0.05 \mathrm{pCi} / \mathrm{g}$ & --- & $5.3 \mathrm{pCi} / \mathrm{g}$ \\
\hline Total Uranium (1974-76) & $1.0 \mathrm{mg} / \mathrm{kg}$ & $1.0 \mathrm{mg} / \mathrm{kg}$ & --- & --- \\
\hline Total Uranium (1977-93) & $0.10 \mathrm{mg} / \mathrm{kg}$ & $0.10 \mathrm{mg} / \mathrm{kg}$ & --- & --- \\
\hline Total Uranium (1994-97) & $0.25 \mathrm{mg} / \mathrm{kg}$ & $0.25 \mathrm{mg} / \mathrm{kg}$ & --- & $93 \mathrm{mg} / \mathrm{kg}$ \\
\hline${ }^{238} \mathrm{Pu}$ & $0.005 \mathrm{pCi} / \mathrm{g}$ & $0.005 \mathrm{pCi} / \mathrm{g}$ & $0.1 \mathrm{fCi} / \mathrm{g}$ & $49 \mathrm{pCi} / \mathrm{g}$ \\
\hline${ }^{239,240} \mathrm{Pu}$ & $0.005 \mathrm{pCi} / \mathrm{g}$ & $0.005 \mathrm{pCi} / \mathrm{g}$ & $0.1 \mathrm{fCi} / \mathrm{g}$ & $44 \mathrm{pCi} / \mathrm{g}$ \\
\hline${ }^{241} \mathrm{Am}$ & $0.005 \mathrm{pCi} / \mathrm{g}$ & $0.005 \mathrm{pCi} / \mathrm{g}$ & --- & $39 \mathrm{pCi} / \mathrm{g}$ \\
\hline Gross Alpha & $1.5 \mathrm{pCi} / \mathrm{g}$ & $1.5 \mathrm{pCi} / \mathrm{g}$ & --- & --- \\
\hline Gross Beta & $1.5 \mathrm{pCi} / \mathrm{g}$ & $1.5 \mathrm{pCi} / \mathrm{g}$ & --- & --- \\
\hline Gross Gamma & $0.8 \mathrm{pCi} / \mathrm{g}$ & $0.8 \mathrm{pCi} / \mathrm{g}$ & --- & --- \\
\hline \multicolumn{5}{|c|}{$\begin{array}{l}\text { a Detection limit as computed by analytical laboratory. See ESP (1998) Table 5-10, p. } 170 . \\
\text { b SAL = screening action level for ER Project, calculated using RESRAD (v. 5.95), Nov. } 2000 \text {; values } \\
\text { are periodically revised. See the ER Radiological Dose Assessment section for the discussion about the } \\
\text { RESRAD model. } \\
\text { Source: ER Project (2001), Analysis and Assessment Focus Area. } \\
\text { c After 1997, the sample size for strontium-90 was reduced to } 2 \text { g because of waste disposal restrictions. }\end{array}$} \\
\hline
\end{tabular}

Sediments in portions of Pueblo, Los Alamos, and Mortandad Canyons have been affected to varying degrees by contaminated releases from the Laboratory. Sediments in these canyons have radioactivity values that are significantly higher than values attributable to worldwide fallout or natural background sources (Graf, 1993 and 1996; Stoker et al., 1981). The presence of contaminated sediments in Pueblo, Los Alamos, and Mortandad Canyons is historically well documented in annual environmental surveillance reports. Some of the Los Alamos Canyon sediments have moved off-site into the Rio Grande. In Mortandad Canyon, the bulk of contaminated sediments have not moved off-site because three sediment traps have prevented sediments from moving toward the eastern Laboratory boundary (Gallaher et al., 1997 and 1999). The remainder of drainages that cross Laboratory lands typically show background radioactivity; however, there are occasional exceptions as seen in the annual environmental surveillance reports.

Historical data from regional sediment stations that are used in this study are reported in the annual environmental surveillance reports. Beginning in 1992, these reports also contain measurements for trace metals, organic compounds, and high-explosive (HE) residuals in sediments, in addition to annual radiochemical data. Historical data from reservoir sediments (Purtymun et al., 1990) are also utilized in the present investigation. In addition, this study incorporates river sediment data from previous reports (Purtymun et al., 1980 and 1987) for the periods 1974-1977 and 1974-1986, respectively. These previous reports combined river sediment and soil analyses data from regional and perimeter sampling stations. However, the present report only focuses on radiochemical analyses of sediments from regional river and reservoir sediment stations. Analyses for fish and soils data are reported elsewhere (Fresquez et al., 1994 and 1996). The regional sediment sampling stations depicted in Figs. 1 and 2 surround the Los Alamos area. Data from these stations provide natural and worldwide fallout information so that regional background values for important radionuclides can be estimated. All of these background data are summarized in Appendices $\mathrm{A}$ and $\mathrm{B}$ of this report. In addition, electronic versions of the data are contained on the 3.5-in. floppy disk attached to the inside back cover of this report. These files are in Microsoft Excel format (PC Windows 
version 5.0). Appendix $C$ contains the computer program used to generate the grouped probability plots for each analyte listed in this report. Appendix D contains the probability plots for grouped river sediment samples, and Appendix E contains the probability plots for grouped reservoir sediment samples.

Worldwide atmospheric nuclear weapons tests were conducted in the 1950s and 1960s by numerous countries (Aarkrog, 1991). Between 1945 and 1963, approximately 360 atmospheric tests were conducted. In 1963, most atmospheric testing ended with the joint US-USSR-UK test ban treaty. However, between 1964 and 1980 approximately 63 atmospheric tests were conducted by China, France, and others. These combined sources have distributed anthropogenetic tritium, strontium-90, cesium-137, plutonium-238, plutonium-239,-240, and americium-241 into the atmosphere, which has resulted in fallout (Norris et al., 1994; Holleman et al., 1987). In 1979, the Three Mile Island Power Station in Pennsylvania released small amounts of radiation into the atmosphere, primarily in the form of radioactive noble gases. In addition, the April 1986 catastrophic failure of Unit 4 at the Chernobyl Nuclear Power Plant in Ukraine released radioactive gases, aerosols, and finely fragmented nuclear fuel particles into the upper atmosphere (Kirhner and Noack, 1988). Finally, between 1964 and 1996, approximately ten nuclear-powered satellites and spacecraft instruments have reentered the earth's atmosphere and burned up (e.g., see http://home.acadia. net/cbm/index.html). Some of these are the SNAP-9A, a US navigational satellite that released 16,200 Ci of plutonium-238 over the Indian Ocean in 1964 (Krey, 1967); the recording seismograph aboard the Apollo 13 lunar module in 1970; the Soviet Kosmos 954 satellite over northwestern Canada in 1978 (Tracy et al., 1984); the Soviet Kosmos 1402 satellite, which released an unknown inventory over the south Atlantic Ocean in 1983; and the Russian Mars space probe, which released 3,200 Ci of plutonium-238 over the Pacific Ocean near Chile in 1996.

A small amount of tritium is also naturally produced by cosmic-ray interaction with the upper atmosphere and is commingled with worldwide fallout. In 1980, it was estimated that about $60 \%$ of the existing plutonium-238 inventory on soils and sediments originated from atmospheric nuclear weapons testing, while the remaining $40 \%$ came from burn-up of satellite power sources upon reentry into the earth's atmosphere (Perkins and Thomas, 1980). Some trace amounts of uranium in sediments can also be attributed to nuclear weapons testing or burn-up of satellite power sources upon atmospheric reentry; however, nearly all uranium in the environment originates from naturally occurring uranium minerals found in the earth's crust. Most of the remaining radiochemical sources examined in this report are anthropogenetic, and are commonly associated with atmospheric weapons testing.

All of the anthropogenetic sources of radionuclide contaminants described above are nonuniformly distributed in time and space. In addition, the point sources described above represent a wide range of radionuclide mass inputs into the global atmosphere. Most of these point inputs cannot be sufficiently quantified. In other words, at the time of introduction into the environment, individual radionuclide mass inputs from individual atmospheric tests or incineration of individual satellites are not completely known. Hence, no correction for radioactive decay was made to any data contained in this report.

\section{SAMPLE COLLECTION PROCEDURES}

Radionuclides naturally tend to bind more readily to clay and silt-sized particles than to sandy portions of a sediment sample. This phenomenon is generally related to strong van der Waals forces, the higher cation exchange capacity, total organic carbon content, and higher surface area of clay and silt particles relative to coarser materials. These finer-grained soil particles, along with any attached radionuclides, are subject to sheet erosion and subsequent overland transport into streams and rivers. Main river channels in the Los Alamos area generally consist of nonhomogeneous mixtures of gravel, sand, silt, and clay. Moisture contents in these channel sediments vary over a wide range because of highly variable stream flows. Finegrained sediments generally occur as dune build-up behind large cobbles and boulders in or along the channel banks. These finer-sized sediments are preferentially collected for analyses because they are more likely to contain detectable radioactivity values. Field sieving of collected samples is not performed because of the possibility of sample cross-contamination. Field sieving is further complicated by variations in moisture contents between sample grain sizes. Instead, sediments are sieved prior to radiochemical analyses under controlled conditions during laboratory sample preparation; the process is discussed below. 
Sediment samples were analyzed for tritium, strontium-90, cesium-137, total uranium, plutonium-238, plutonium-239,-240, americium-241, gross alpha, gross beta, and gross gamma radioactivity. A brief description of sample preparation and analyses is presented here. Detailed descriptions of these procedures are found elsewhere (Gautier and Gladney, 1993; Environmental Surveillance Program, 1996). Table 1 briefly summarizes the typical limits of detection for individual analytes used in this report.

Annual river sediment samples are collected along the banks of the main channels of the Rio Grande, Rio Chama, and Jemez River. Samples from the channel beds of intermittently flowing streams are collected across the main channel to a depth of about $5 \mathrm{~cm}$. Prior to 1994, individual river sediment samples were approximately $250 \mathrm{~g}$. However, laboratory aliquots of only $10 \mathrm{~g}$ were randomly selected from these samples for actual radionuclide analyses. After 1994, these river samples were reduced to approximately $100 \mathrm{~g}$, while the laboratory aliquots for all analytes remained at $10 \mathrm{~g}$. In 1997 the strontium-90 aliquots were further reduced to $2 \mathrm{~g}$ because of laboratory waste disposal requirements.

Reservoir sediments are collected annually from a boat using an Eckman dredge that collects a sample of sediments from a square area approximately $15 \mathrm{~cm}(6 \mathrm{in}$.) on a side and to a depth of approximately $5 \mathrm{~cm}$ ( 2 in.). Sufficient sediments are collected at each station so that the analyzed samples are at least $1 \mathrm{~kg}$. These larger reservoir samples yield lower detection limits for plutonium analyses. Detection limits for other radionuclides remain unchanged from those of river sediment samples because sample aliquots from both river and reservoir samples are identical. These reservoir sediment samples are representative of annual sediment inflows into the reservoirs (and annual radionuclide inventory influxes) because they are collected from the top of the bottom sediment surface. Furthermore, reservoir sediment stations are approximately located over stream channels that existed prior to reservoir construction. These sampling locations were selected because sedimentation rates tend to be a maximum here. Based on US Army Corps of Engineers' sonic bottom profiles, sedimentation rates in large New Mexico reservoirs typically range from about 10 to $60 \mathrm{~cm} / \mathrm{yr}$. The sampled reservoirs discussed in this report typically show a sedimentation rate exceeding $25-30 \mathrm{~cm} / \mathrm{yr}$ in the channel portions of the reservoir. However, the US Geological Survey recently reported an average sedimentation rate of only $6.6 \mathrm{~cm} / \mathrm{yr}$ at one of its sampling sites in Cochiti Reservoir (Wilson and van Metre, 2000).

For the purposes of this study, the dredge sediment sampling technique is preferred over core sampling because cores typically penetrate through multiple sediment layers representing several years of combined sediment inflows into a reservoir. One significant implication of dredge versus core sampling is estimating annual and total radionuclide inventories in individual reservoirs. For a given reservoir, the annual series of radioactivity values tabulated here from dredged samples can be used to estimate both the annual and total radionuclide inventories in that reservoir if the annual volumetric influx of sediments can be estimated. The US Army Corps of Engineers or US Bureau of Reclamation typically perform bottom-profile surveys every few years to measure these sedimentation rates. Radioactivity values on sediments from cored samples typically represent vertical averages over an unknown depositional time interval. Hence, these data can only be used to estimate total inventory accumulations up to the time of core recovery. Consequently, important information describing the spatial and temporal variability between annual inflow events and the radionuclide inventory in sediments may be smeared out. Thus, significant information may be lost with the core sampling technique; this information can be used to describe the dynamic inflow-outflow relationship between annual water and sediment influx. However, the core technique can be very useful if reservoir sediments are infrequently sampled. In addition, at least three time-deposition markers can be used with core samples to establish a general chronology (i.e., sediment surface, sediment bottom, and the cesiun-137 peak associated with atmospheric weapons testing). Both core and dredge samples have uncertainty to their depositional histories, and neither technique fills all needs. Core techniques are applicable to infrequently sampled reservoirs, while dredge sampling can have significantly lower detection limits. 


\section{METHODS OF ANALYSES}

Prior to oven-drying at about $70^{\circ} \mathrm{C}\left(160^{\circ} \mathrm{F}\right)$, a portion of the sediment sample (river or reservoir) was gently heated and distilled moisture was collected. Captured water that was distilled from the sediment sample was then mixed with a counting cocktail and analyzed for tritium by liquid scintillation counting. The remaining sediments in the sample were then oven-dried, sieved through a $1.7-\mathrm{mm}$ screen, and thoroughly blended. This sieving removes cobble-sized particles and plant debris but does not separate sand from silt- and clay-sized particles. For each river sediment sample, a separate 10-g aliquot was taken for each analysis, while the entire 1-kg sediment sample was used for the reservoir analysis.

Prior to 1997, samples for strontium-90 analyses were completely dissolved in nitric acid, then extracted with bis(2-ethylhexyl) hydrogen phosphate to remove yttrium-90 daughters and interfering radionuclides. Stable yttrium was then added as a chemical tracer. After allowing the yttrium-90 daughters to equilibrate with strontium-90, the sample was then extracted, the yttrium purified, and the sample was beta-counted on a gas-proportional counter. This method assumes $100 \%$ tracer recovery. In 1997, the sample preparation procedure was modified so that sample tracer recoveries could be measured. This modified procedure passes the acidified sample through an Empore strontium radiation disk to quantitatively extract the strontium. The disk is then counted for beta radioactivity using liquid scintillation counting.

Samples for cesium-137 were counted directly with a Ge-Li detector coupled with a multichannel analyzer. Aliquots (10 g for river sediments and $1 \mathrm{~kg}$ for lake-bottom sediments) for plutonium-238 and plutonium-239,-240 analyses were spiked with plutonium-242 tracers and completely dissolved in nitric and hydrofluoric acid. Plutonium was isolated by anion exchange and electroplated onto stainless steel disks. The disks were then counted on an alpha spectrometer.

Total uranium analyses from 1974 through 1976 were performed using a fluorometric method. Total uranium analyses from 1976 through 1992 were performed by irradiating the samples with epithermal neutrons, followed by counting on a Ge-Li gamma-ray spectrometer. Beginning in 1993, the kinetic phosphorescence analysis (KPA) of uranium was employed. These changes in analytical methods for total uranium resulted in changes in the limits of detection from 1974 through 1997 (see Table 1). These detection limit changes have not affected our analyses because reported total uranium concentration levels in sediments have always been greater than any of these detection limit values.

Americium-241 analyses from 1976 to 1997 were performed using radiochemistry and alpha spectrometry. Prior to 1997 , samples were initially screened with gamma-ray spectrometry; however, that step was eliminated. Now samples are only subjected to chemical separation and alpha spectrometry.

Measurements of radioactivity in samples require that analytical or instrumental background values be subtracted from recorded values to obtain net radioactivity values in the samples. Thus, sample radioactivity values are sometimes obtained that are less than the typical detection limit of the analytical technique. This situation is due to numerous factors, including electrical noise in the instrument counting procedure for samples with little or no radioactivity. Consequently, individual measurements for a given radionuclide can result in values of positive or negative numbers. Although a negative number does not represent a physical reality, a valid long-term average of many measurements can be obtained only if the very small positive and negative values are included in the population calculations (Gilbert, 1975). Hence, any originally reported negative values from the laboratory are retained for the evaluation of background radioactivity. Small positive values that are below the reported detection limits (Table 1) are also retained for the same reason.

For this report, all radionuclide data for river and reservoir sediments collected from 1974 through 1997 were obtained from original field sampling log books and their related laboratory analytical report sheets in lieu of downloading information directly from the Laboratory's electronic database. This complete manual archival search was conducted after several discrepancies in radionuclide values were discovered in a routine comparison of randomly selected data values from several different published sources. These occasional discrepancies between data sources seem to occur before about 1990. Data sources for this comparison included the annual environmental surveillance reports, the Purtymun et al. (1987) report, and the Laboratory Information Management System (LIMS) electronic database. The reader should be aware that all radionuclide values used in this study for river and reservoir sediments have been thoroughly verified with original laboratory data sources. Furthermore, the data in this report may not always be identical to historical data reported elsewhere because of occasional typographical errors in these earlier sources. No changes have been made to published historical data however. In conclusion, the data contained in this report represent the most accurate information available and should be used in lieu of historically published data. 


\section{REVIEW OF SEDIMENT DATA}

A detailed review of the tabulated data contained in Appendix A (river sediments) and Appendix B (reservoir sediments) reveals some important departures from anticipated results for individual sample values. These data are highlighted in the appendices because they qualify as statistical outliers or because laboratory procedures for sample preparation were not followed. These highlighted data were not used in this statistical analysis to determine the upper limit of background radioactivity values for river and reservoir sediments. Table 2 summarizes the criteria used to review and eliminate data from statistical evaluation.

Table 2. Criteria Used for Data Elimination.

\begin{tabular}{|c|c|c|c|c|}
\hline Number & Analyte & Year & Station(s) & $\begin{array}{l}\text { Criteria for Data Elimination } \\
\text { (see text for details) }\end{array}$ \\
\hline \multicolumn{5}{|c|}{$\begin{array}{l}\text { Group A Errors: } \\
\text { systematic laborat }\end{array}$} \\
\hline 1 & ${ }^{90} \mathrm{Sr}$ & $1997^{\mathrm{a}}$ & All & Change in laboratory analytical procedure. \\
\hline 2 & ${ }^{90} \mathrm{Sr}$ & $1996^{\mathrm{a}}$ & Cochiti Reservoir & Change in laboratory analytical procedure. \\
\hline 3 & ${ }^{238} \mathrm{Pu}$ & 1995 & All Reservoir & $\begin{array}{l}\text { Error in laboratory sample preparation } \\
\text { (used } 10-\mathrm{g} \text { rather than } 1-\mathrm{kg} \text { sample). }\end{array}$ \\
\hline 4 & ${ }^{239}, 240 \mathrm{Pu}$ & 1995 & All Reservoir & $\begin{array}{l}\text { Error in laboratory sample preparation } \\
\text { (used } 10-\mathrm{g} \text { rather than } 1-\mathrm{kg} \text { sample). }\end{array}$ \\
\hline 5 & ${ }^{241} \mathrm{Am}$ & $1992^{b}$ & All River & $\begin{array}{l}\text { Unknown systematic lab error; } \\
\text { large positive values and uncertainties reported. }\end{array}$ \\
\hline 6 & ${ }^{241} \mathrm{Am}$ & 1992 & All Reservoir & $\begin{array}{l}\text { Unknown systematic lab error; } \\
\text { large negative values and uncertainties reported. }\end{array}$ \\
\hline 7 & ${ }^{241} \mathrm{Am}$ & 1987 & All River & $\begin{array}{l}\text { Unknown systematic lab error; } \\
\text { large negative values and uncertainties reported. }\end{array}$ \\
\hline 8 & ${ }^{241} \mathrm{Am}$ & 1978 & All River & $\begin{array}{l}\text { Unknown systematic lab error; } \\
\text { large negative values and uncertainties reported. }\end{array}$ \\
\hline 9 & ${ }^{90} \mathrm{Sr}$ & 1976 & All River & $\begin{array}{l}\text { Unknown systematic lab error; } \\
\text { large positive values and uncertainties reported. }\end{array}$ \\
\hline 10 & ${ }^{3} \mathrm{H}$ & $<1987$ & All & $\begin{array}{l}\text { Atmospheric releases before } 1987 \text {; } \\
\text { possible sample and lab contamination. }\end{array}$ \\
\hline
\end{tabular}

Group B Errors:

apparent reporting errors at individual sample stations.

\begin{tabular}{|c|c|c|c|c|}
\hline 11 & ${ }^{3} \mathrm{H}$ & 1997 & $\begin{array}{l}\text { Heron Reservoir } \\
\quad(\text { middle })^{\mathrm{c}}\end{array}$ & Outlier; reported value greater than $(x+5 s) .^{d}$ \\
\hline 12 & ${ }^{3} \mathrm{H}$ & 1996 & Frijoles & Outlier; reported value greater than $(x+5 s){ }^{d}$ \\
\hline 13 & ${ }^{90} \mathrm{Sr}$ & 1995 & Chamita & Outlier; reported value greater than $(x+5 s){ }^{d}$ \\
\hline 14 & ${ }^{137} \mathrm{Cs}$ & 1994 & Otowi & Outlier; reported value greater than $(x+5 s) .{ }^{d}$ \\
\hline 15 & ${ }^{90} \mathrm{Sr}$ & 1994 & Ancho & Outlier; reported value greater than $(x+5 s) .{ }^{d}$ \\
\hline 16 & ${ }^{90} \mathrm{Sr}$ & 1994 & Chamita & Outlier; reported value greater than $(x+5 s) .{ }^{d}$ \\
\hline 17 & Total U & 1993 & Frijoles & Outlier; reported value greater than $(x+5 s){ }^{d}$ \\
\hline 18 & ${ }^{90} \mathrm{Sr}$ & 1989 & $\begin{array}{l}\text { Abiquiu Reservoir } \\
{\text { (middle })^{\mathrm{c}}}^{\text {(mir }}\end{array}$ & Outlier; reported value greater than $(x+5 s) .{ }^{d}$ \\
\hline 19 & ${ }^{239,240} \mathrm{Pu}$ & 1982 & Pajarito & Outlier; reported value greater than $(x+5 s) .{ }^{d}$ \\
\hline 20 & ${ }^{90} \mathrm{Sr}$ & 1981 & Bernalillo & Outlier; reported value greater than $(x+5 s){ }^{d}$ \\
\hline 21 & ${ }^{90} \mathrm{Sr}$ & 1979 & Embudo & Outlier; reported value greater than $(x+5 s){ }^{d}$ \\
\hline
\end{tabular}

a All samples collected after September 1996 are affected.

b Samples collected between September and December 1992 are not affected.

c See the discussion about the station locations in the Reservoir Sediments subsection of the Results section.

${ }^{\mathrm{d}}$ Value exceeds analyte mean (x) plus five times analyte standard deviation (s), or ( $\left.\mathrm{x}+5 \mathrm{~s}\right)$; see Appendix A or B. 
Elimination of questionable data tends to lower the respective means and standard deviations of individual radionuclides. The majority of the suspect data eliminated from analyses included tritium (pre-1987 samples), strontium-90 (especially 1976 and 1997 data), plutonium (only 1995 data), and americium-241 (especially 1987 and 1992 data). Sometimes, all suspect data at all sediment stations were eliminated from statistical analyses for a particular analyte in a given year. In other cases, only individual analytes were eliminated because questions involved only individual batch samples for that analyte. For example, prior to the mid 1980s, unreliable tritium measurements were reported (Rogers, 1998, pp. 8 and 9) because of possible airborne releases at the Laboratory that resulted in cross-contamination of samples during analyses. Hence, all tritium samples collected prior to 1987 were eliminated from this study. In addition, the sample preparation procedure for strontium-90 analysis was modified in October 1996. This change has resulted in changes in all reported strontium-90 values made after this date. Hence, some 1996 and all 1997 strontium90 data were not used in the statistical analyses reported here. In 1995, errors in sample preparation procedures for reservoir plutonium analyses were made. Hence, all 1995 reservoir samples for plutonium were eliminated from statistical analyses, while 1995 river sediment analyses for plutonium were retained. Similar comments can be made for 1987 and 1992 americium-241 batch samples. Hence, only certain americium-241 sample results were eliminated from statistical analyses depending on sample submission dates. Less than 4\% of all historical river sediment analyses performed between 1974 and 1997 were dropped from the statistical analyses. Less than $6 \%$ of the reservoir sediment samples collected between 1979 and 1997 were eliminated. Table 3 gives a comprehensive breakdown of sediment samples that were reviewed to ensure that laboratory quality assurance and quality control (QA/QC) guidelines were satisfied. Finally, all data are contained in Appendices A and B (odd-numbered Tables A-1 through A-19 and B-1 through B-9), including those data that were eliminated from statistical analyses reported in this study. Inclusion of all data was done for historical completeness. Data that do not meet minimum laboratory QA/QC guidelines (i.e., the highlighted values in Appendices A and B) should not be used in future studies.

As mentioned above, the sample preparation procedure for strontium-90 analysis was modified in October 1996. This change affected the 1996 samples collected from Cochiti Reservoir, and all 1997 sediment samples. In addition, the change has resulted in higher detected values and uncertainties for all strontium-90 analyses because of improvements in laboratory tracer recoveries. Previously, tracer recovery values for strontium-90 analyses were assumed to be $100 \%$. Hence, pre-1997 strontium-90 values (and the 1996 strontium-90 values from Cochiti Reservoir samples) may be artificially low relative to post-1997 data. In the future, all strontium-90 values need to be segregated into pre-1997 and post-1997 groups because of possible nonstationarity in the computed means and standard deviations. For this report, however, all strontium-90 sediment data collected in 1997 were simply eliminated from statistical analyses. After four to five annual sediment samples have been collected and new post-1997 strontium-90 values are available, then a new background radioactivity value for strontium-90 should be computed using only post-1997 data. The upper limit of this post-1997 strontium-90 mean should be compared with only post-1997 strontium-90 data. Likewise, the pre-1997 background value reported here for strontium-90 in sediments should only be compared with pre-1997 data. Finally, a potential future increase in mean strontium-90 values computed from post-1997 data should not be interpreted as an upward trend in background radioactivity (or an upward trend in values from on-site sediment stations). Instead, these changes simply reflect the change in laboratory analytical procedures for this analyte.

\section{DESCRIPTION OF SEDIMENT SAMPLING STATIONS}

\section{River Sediment Stations}

Ten river sediment stations have historically been included as background sampling locations for the Laboratory's environmental surveillance network. These stations include the Rio Chama at Chamita, the Rio Grande at Embudo, the Rio Grande at Otowi Bridge, the Rio Grande below Sandia Canyon, the Rio Grande below Pajarito Canyon, the Rio Grande below Ancho Canyon, the Rio Grande below Frijoles Canyon, the Rio Grande below Cochiti Reservoir Spillway, the Rio Grande at Bernalillo, and the Jemez River near Jemez Pueblo (Figs. 1 and 2). Historical river sediment data from these stations are tabulated in Appendix A (odd numbered Tables A-1 through A-19), including statistical summaries for each station (even numbered Tables A-2 through A-20). 
Table 3. Total Number of Sediment Samples Reviewed by ESH-18.

\begin{tabular}{|c|c|c|c|c|c|c|c|c|c|c|c|c|c|c|c|}
\hline \multirow{2}{*}{$\begin{array}{c}\text { Group I } \\
\text { River } \\
\text { Stations } \\
\end{array}$} & \multicolumn{3}{|c|}{$\underline{\text { Rio Chama at Chamita }}$} & \multicolumn{3}{|c|}{$\underline{\text { Rio Grande at Embudo }}$} & \multicolumn{3}{|c|}{$\begin{array}{l}\text { Rio Grande below } \\
\text { Cochiti Spillway }\end{array}$} & \multicolumn{3}{|c|}{$\begin{array}{r}\text { Rio Grande } \\
\text { at Bernalillo }\end{array}$} & \multicolumn{3}{|c|}{ Jemez River } \\
\hline & Pass & Fail & Total & Pass & Fail & Total & Pass & Fail & Total & Pass & Fail & Total & Pass & Fail & Total \\
\hline${ }^{3} \mathrm{H}$ & 6 & 2 & 8 & 6 & 3 & 9 & 1 & 1 & 2 & 6 & 2 & 8 & 5 & 2 & 7 \\
\hline${ }^{90} \mathrm{Sr}$ & 10 & 4 & 14 & 11 & 3 & 14 & 3 & 1 & 4 & 10 & 3 & 13 & 9 & 1 & 10 \\
\hline${ }^{137} \mathrm{Cs}$ & 23 & 0 & 23 & 24 & 0 & 24 & 6 & 0 & 6 & 23 & 0 & 23 & 23 & 0 & 23 \\
\hline Total U & 19 & 0 & 19 & 20 & 0 & 20 & 5 & 0 & 5 & 20 & 0 & 20 & 19 & 0 & 19 \\
\hline${ }^{238} \mathrm{Pu}$ & 26 & 0 & 26 & 27 & 0 & 27 & 8 & 0 & 8 & 24 & 0 & 24 & 26 & 0 & 26 \\
\hline${ }^{239,240} \mathrm{Pu}$ & 26 & 0 & 26 & 27 & 0 & 27 & 8 & 0 & 8 & 24 & 0 & 24 & 26 & 0 & 26 \\
\hline${ }^{241} \mathrm{Am}$ & 6 & 2 & 8 & 4 & 2 & 6 & 1 & 0 & 1 & 6 & 2 & 8 & 5 & 1 & 6 \\
\hline G. Alpha & 15 & 0 & 15 & 15 & 0 & 15 & 6 & 0 & 6 & 16 & 0 & 16 & 16 & 0 & 16 \\
\hline G. Beta & 15 & 0 & 15 & 15 & 0 & 15 & 6 & 0 & 6 & 16 & 0 & 16 & 16 & 0 & 16 \\
\hline G. Gamma & 20 & 0 & 20 & 19 & 0 & 19 & 4 & 0 & 4 & 19 & 0 & 19 & 19 & 0 & 19 \\
\hline Totals & 166 & 8 & 174 & 168 & 8 & 176 & 48 & 2 & 50 & 164 & 7 & 171 & 164 & 4 & 168 \\
\hline \multirow{2}{*}{$\begin{array}{c}\text { Group II } \\
\text { River } \\
\text { Stations } \\
\end{array}$} & \multicolumn{3}{|c|}{ Rio Grande at Otowi } & \multicolumn{3}{|c|}{$\begin{array}{c}\text { Rio Grande } \\
\text { below Sandia }\end{array}$} & \multicolumn{3}{|c|}{$\begin{array}{c}\text { Rio Grande } \\
\text { below Pajarito }\end{array}$} & \multicolumn{3}{|c|}{$\begin{array}{c}\text { Rio Grande } \\
\text { below Ancho }\end{array}$} & \multicolumn{3}{|c|}{$\begin{array}{c}\text { Rio Grande } \\
\text { below Frijoles }\end{array}$} \\
\hline & Pass & Fail & Total & Pass & Fail & Total & Pass & Fail & Total & Pass & Fail & Total & Pass & Fail & Total \\
\hline${ }^{3} \mathrm{H}$ & 6 & 2 & 8 & 6 & 0 & 6 & 6 & 0 & 6 & 5 & 0 & 5 & 7 & 3 & 10 \\
\hline${ }^{90} \mathrm{Sr}$ & 12 & 1 & 13 & 4 & 0 & 4 & 4 & 0 & 4 & 4 & 1 & 5 & 9 & 2 & 11 \\
\hline${ }^{137} \mathrm{Cs}$ & 24 & 1 & 25 & 16 & 0 & 16 & 16 & 0 & 16 & 15 & 0 & 15 & 20 & 0 & 20 \\
\hline Total U & 20 & 0 & 20 & 14 & 0 & 14 & 13 & 0 & 13 & 12 & 0 & 12 & 16 & 1 & 17 \\
\hline${ }^{238} \mathrm{Pu}$ & 27 & 0 & 27 & 17 & 0 & 17 & 16 & 0 & 16 & 17 & 0 & 17 & 23 & 0 & 23 \\
\hline $239,240 \mathrm{Pu}$ & 27 & 0 & 27 & 17 & 0 & 17 & 15 & 1 & 16 & 17 & 0 & 17 & 23 & 0 & 23 \\
\hline${ }^{241} \mathrm{Am}$ & 5 & 3 & 8 & 2 & 0 & 2 & 2 & 0 & 2 & 2 & 0 & 2 & 5 & 0 & 5 \\
\hline G. Alpha & 16 & 0 & 16 & 7 & 0 & 7 & 7 & 0 & 7 & 7 & 0 & 7 & 14 & 0 & 14 \\
\hline G. Beta & 16 & 0 & 16 & 7 & 0 & 7 & 7 & 0 & 7 & 7 & 0 & 7 & 13 & 0 & 13 \\
\hline G. Gamma & 20 & 0 & 20 & 6 & 0 & 6 & 6 & 0 & 6 & 6 & 0 & 6 & 15 & 0 & 15 \\
\hline Totals & 173 & 7 & 180 & 96 & 0 & 96 & 92 & 1 & 93 & 92 & 1 & 93 & 145 & 6 & 151 \\
\hline \multirow{2}{*}{$\begin{array}{c}\text { Reservoir } \\
\text { Stations } \\
\end{array}$} & \multicolumn{3}{|c|}{ Abiquiu Reservoir } & \multicolumn{3}{|c|}{ El Vado Reservoir } & \multicolumn{3}{|c|}{ Heron Reservoir } & \multicolumn{3}{|c|}{ Rio Grande Reservoir } & \multicolumn{3}{|c|}{ Cochiti Reservoir } \\
\hline & Pass & Fail & Total & Pass & Fail & Total & Pass & Fail & Total & Pass & Fail & Total & Pass & Fail & Total \\
\hline${ }^{3} \mathrm{H}$ & 23 & 0 & 23 & 6 & 0 & 6 & 8 & 1 & 9 & 6 & 0 & 6 & 24 & 0 & 24 \\
\hline${ }^{90} \mathrm{Sr}$ & 29 & 3 & 32 & 6 & 3 & 9 & 9 & 3 & 12 & 3 & 3 & 6 & 24 & 6 & 30 \\
\hline${ }^{137} \mathrm{Cs}$ & 32 & 0 & 32 & 12 & 0 & 12 & 15 & 0 & 15 & 9 & 0 & 9 & 33 & 0 & 33 \\
\hline Total U & 32 & 0 & 32 & 12 & 0 & 12 & 15 & 0 & 15 & 9 & 0 & 9 & 30 & 0 & 30 \\
\hline${ }^{238} \mathrm{Pu}$ & 40 & 3 & 43 & 15 & 3 & 18 & 18 & 3 & 21 & 10 & 3 & 13 & 45 & 3 & 48 \\
\hline${ }^{239,240} \mathrm{Pu}$ & 40 & 3 & 43 & 15 & 3 & 18 & 18 & 3 & 21 & 10 & 3 & 13 & 45 & 3 & 48 \\
\hline${ }^{241} \mathrm{Am}$ & 17 & 3 & 20 & 9 & 0 & 9 & 12 & 0 & 12 & 9 & 0 & 9 & 21 & 3 & 24 \\
\hline G. Alpha & 17 & 0 & 17 & 9 & 0 & 9 & 12 & 0 & 12 & 9 & 0 & 9 & 18 & 0 & 18 \\
\hline G. Beta & 17 & 0 & 17 & 9 & 0 & 9 & 12 & 0 & 12 & 9 & 0 & 9 & 18 & 0 & 18 \\
\hline G. Gamma & 26 & 0 & 26 & 9 & 0 & 9 & 12 & 0 & 12 & 9 & 0 & 9 & 24 & 0 & 24 \\
\hline Totals & 273 & 12 & 285 & 102 & 9 & 111 & 131 & 10 & 141 & 83 & 9 & 92 & 282 & 15 & 297 \\
\hline
\end{tabular}


The Chamita sampling station lies approximately $30 \mathrm{~km}$ (19 mi) downstream from Abiquiu Reservoir on the Rio Chama (Fig. 1). Samples were collected from the site from 1974 through 1997. The Embudo sampling station lies on the Rio Grande approximately $20 \mathrm{~km}$ (12 mi) upstream from the confluence with the Rio Chama. Samples were collected at the site from 1974 through 1997. The Otowi sediment sampling station is located on the Rio Grande approximately $20 \mathrm{~km}(12 \mathrm{mi})$ south of the confluence with the Rio Chama (Fig. 2). Samples were collected from 1974 through 1997. The Sandia sampling station is located on the Rio Grande just below the confluence with Sandia Canyon and is approximately $6 \mathrm{~km}$ (4 mi) downstream from the Otowi site. Samples have been collected from 1978 through 1994. The Pajarito sampling station is located on the Rio Grande just below the confluence with Pajarito Canyon, and is approximately $4 \mathrm{~km}$ (2.5 mi) downstream from the Sandia sampling site. Samples were collected at Pajarito from 1978 through 1994. The Ancho sampling station is located on the Rio Grande just below the confluence with Ancho Canyon, and is approximately $4 \mathrm{~km}(2.5 \mathrm{mi})$ downstream from the Pajarito sampling site. Samples were collected at Ancho from 1978 through 1994. The Frijoles sampling station is located on the Rio Grande just below the confluence with Frijoles Canyon, and is approximately $4 \mathrm{~km}$ (2.5 mi) downstream from the Ancho sampling site. Samples were collected from 1976 through 1997. The Cochiti Spillway sampling station is located on the Rio Grande immediately downstream from the dam at Cochiti Reservoir. Samples were collected from 1974 to 1979 and in 1995. The Bernalillo sediment-sampling site is located on the Rio Grande approximately $20 \mathrm{~km}$ (12 mi) downstream from the confluence with the Jemez River. Samples were collected from 1974 through 1997. The Jemez River sediment sampling site is located on the Jemez River approximately $50 \mathrm{~km}$ (31 mi) upstream from the confluence with Rio Grande (Fig. 1). Samples were collected from 1974 through 1997. Mean daily discharge characteristics from US Geological Survey (USGS) stream gaging stations that are located near these sampling stations are summarized in Table 4.

\section{Reservoir Sediment Stations}

It is well known that reservoir construction on a river dramatically alters channel flow velocity and sediment transport capacity (Glymph, 1973). Channel sediment loads are derived from upstream sheet and bank erosion. Sediment deposition in reservoirs depends on several factors. These include (1) the grain-size distribution of the sediment, (2) stream-channel and reservoir inflow-outflow relationships, (3) water temperature gradients, (4) geometric shape of the reservoir, and (5) reservoir operation. Generally, there is a sudden decrease in river velocity and turbulence upon entering a reservoir. This causes entrained and suspended particles to be deposited on the reservoir bottom. Initially, the larger particles are deposited first, while the finer sediments are transported farther downstream in the reservoir before deposition. Due to the generally high cation exchange capacity and surface area of finer particles, one might expect to find higher radioactivity levels on sediments in the lower reaches of a reservoir. Furthermore, one might also expect to find higher radioactivity in reservoir sediments compared with river sediments because of the finer grainsize distributions in reservoirs. Actual sediment transport behavior is more complex, however, and spatial and temporal variability in the data are common. Factors influencing this variability include changes in sediment transport rates, surficial geology, grain size, background radioisotope deposition, and sedimentsampling location relative to the reservoir boundary or former stream channels.

Sediment samples from five regional reservoirs have historically been included as background sampling locations for the Laboratory's environmental surveillance network. These reservoir sampling stations are located in northern New Mexico at Abiquiu, El Vado, and Heron Reservoirs on the Rio Chama, in southern Colorado at the Rio Grande Reservoir on the Rio Grande, and in northern New Mexico at Cochiti Reservoir on the Rio Grande (Fig. 1). Each reservoir actually has three sample collection stations (i.e., an upper, middle, and lower station). Historical reservoir sediment data from these stations are tabulated in Appendix B (odd numbered Tables B-1 through B-9), including statistical summaries for each reservoir (even numbered Tables B-2 through B-10).

Sediments are collected annually from all five reservoirs during the late summer or early fall. Table 5 summarizes some characteristic reservoir information that may influence sedimentation rates. Heron Reservoir is located on Willow Creek just above its confluence with the Rio Chama about $16 \mathrm{~km}(10 \mathrm{mi})$ west of Tierra Amarilla. It is used for storage of San Juan Chama Project water by the US Bureau of Reclamation. Portions of these waters are captured west of the continental divide in the San Juan Mountains of southern Colorado. Captured waters are then conveyed into the Heron watershed by an aqueduct as part of the 
Table 4. Mean Daily Discharge at Selected USGS Gages. See Web Site http://h2o.usgs.gov.

\begin{tabular}{|c|c|c|c|c|c|}
\hline USGS Gage Name & USGS Gage Number & Drainage Area $\left(\mathbf{m i}^{\mathbf{2}}\right)$ & Elevation $(\mathbf{f t})^{\mathbf{a}}$ & Mean Daily Flow (cfs) & Std Dev $(\mathrm{cfs})^{b}$ \\
\hline Rio Chama near Chamita & 8290000 & 3144 & 5654 & 543 & 743 \\
\hline Rio Grande near Embudo & 8279500 & 10400 & 5789 & 913 & 1184 \\
\hline Rio Grande at Otowi Bridge & 8313000 & 14300 & 5488 & 1500 & 1826 \\
\hline Rio Grande below Cochiti Dam & 8317400 & 14900 & 5226 & 1444 & 1479 \\
\hline Rio Grande at Bernalillo & 8329500 & 17300 & 5031 & 1076 & 1599 \\
\hline Jemez River near Jemez Pueblo & 8324000 & 470 & 5622 & 79 & 147 \\
\hline
\end{tabular}


Table 5. Summary of Important Reservoir Sedimentation Characteristics. See National Inventory of Dams Database and Web Site for Additional Information (http://npdp.stanford.edu).

\begin{tabular}{lccccc}
\hline & Heron & El Vado & Abiquiu & Rio Grande & Cochiti \\
\hline Year Constructed & 1971 & 1935 & 1963 & 1890 & 1975 \\
National ID Number & NM-00122 & NM-00127 & NM-00001 & CO-00805 & NM-00404 \\
River System & Rio Chama & Rio Chama & Rio Chama & Rio Grande & Rio Grande \\
North Latitude & $36^{\circ} 39^{\prime} 58^{\prime \prime}$ & $36^{\circ} 35^{\prime} 30^{\prime \prime}$ & $36^{\circ} 14^{\prime} 24^{\prime \prime}$ & $37^{\circ} 43^{\prime} 18^{\prime \prime}$ & $35^{\circ} 37^{\prime} 30^{\prime \prime}$ \\
West Longitude & $106^{\circ} 42^{\prime} 36^{\prime \prime}$ & $106^{\circ} 43^{\prime} 48^{\prime \prime}$ & $106^{\circ} 25^{\prime} 48^{\prime \prime}$ & $107^{\circ} 16^{\prime} 00^{\prime \prime}$ & $1^{\circ} 6^{\circ} 20^{\prime} 00^{\prime \prime}$ \\
Spillway Elevation (ft above stream) & 254 & 175 & 319 & 111 & 246 \\
Drainage Area (mi $\left.{ }^{2}\right)$ & $193^{\mathrm{a}}$ & 868 & 2,146 & 169 & 14,635 \\
Maximum Storage Volume (ac-ft) & 429,646 & 219,580 & $1,369,000$ & 73,800 & 722,000 \\
Normal Storage Volume (ac-ft) & 401,317 & 186,250 & 170,000 & 52,192 & 50,130 \\
Dam Type & Earth Fill & Earth Fill & Earth Fill & Earth Fill & Earth Fill \\
& & & & & \\
\hline
\end{tabular}

a Includes drainage areas for San Juan Chama Project diversion waters. 
diversion project. Sediment samples were collected in 1982, 1984, 1985, and 1994 through 1997. El Vado Reservoir, located on the Rio Chama near El Vado, was completed in 1935. This reservoir is located immediately downstream from Heron Reservoir. Sediment samples were collected in 1982, 1984, 1985, and 1995 through 1997. Abiquiu Reservoir, which is on the Rio Chama downstream from El Vado Reservoir, was completed in 1963. Reservoir sediments were sampled in 1982 and 1984 through 1997. Rio Grande Reservoir, which is the farthest upstream of the reservoirs in this study, was completed in 1890. It is one of the oldest reservoirs in the Rio Grande drainage basin. The reservoir's drainage area is located in the San Juan Mountains east of the continental divide on lands surrounding the reservoir. The elevation of the reservoir exceeds $9,400 \mathrm{ft}$ (2,870 m). The reservoir's upper, middle, and lower reaches were sampled in 1982, 1986, and 1995 through 1997.

Cochiti Reservoir, southeast of Los Alamos, is one of the newest reservoirs along the Rio Grande. The drainage area encompasses portions of southern Colorado and northern New Mexico. This reservoir collects water and sediment from both the Rio Grande and Rio Chama. It is the only reservoir used in this study that is located downstream of the Laboratory boundary. In 1979 and in 1982, sediments from the reservoir were sampled at seven locations from the inlet downstream to the dam (Purtymun et al., 1990; Gallaher et al., 1999). For our purposes, only sites 1, 4, and 7 from those original studies are reported here because these sites correspond to our upper, middle, and lower sediment-sampling stations for the reservoir. For the remaining years (1984 through 1997), Cochiti Reservoir was only sampled at the upper, middle, and lower sediment stations.

\section{STATISTICAL ANALYSES}

Sediment samples from the ten river stations and five reservoirs represented by the data in this report have been historically viewed as Laboratory background stations. These data have always been blended together, and background radioactivities have been computed from these blended data. This raises a fundamentally important question: Does this practice of blending data influence the background radioactivity values for individual analytes? In order to answer this question, the river and reservoir sediments were initially segregated. Then the river and reservoir groups were further subdivided into upstream (Group I) and downstream (Group II) stations. Here the upstream and downstream designation refers to the station location relative to surface drainage of Laboratory lands (Figs. 1 and 2). Hence, the Group I (River) stations consist of sediment samples from the Rio Chama at Chamita, the Rio Grande at Embudo, the Rio Grande below Cochiti Spillway, the Rio Grande at Bernalillo, and the Jemez River. All of the Group I (River) stations are located upstream of the Laboratory except the stations on the Rio Grande below Cochiti Spillway and at Bernalillo. However, both of these stations are located below Cochiti Reservoir (where sediments tend to become trapped). The Group II (River) stations consist of sediment samples from the Rio Grande at Otowi, the Rio Grande below the Sandia Canyon confluence, the Rio Grande below the Pajarito Canyon confluence, the Rio Grande below the Ancho Canyon confluence, and the Rio Grande below the Frijoles Canyon confluence. All of these Group II (River) stations are located downstream of the Laboratory and upstream of Cochiti Reservoir.

Using similar reasoning, sediment samples from reservoirs were segregated into upstream and downstream groups. The upstream Group I (Reservoirs) included sediment samples from Abiquiu, El Vado, and Heron Reservoirs on the Rio Chama of northern New Mexico, and Rio Grande Reservoir on the Rio Grande in southern Colorado. The downstream Group II (Reservoirs) only included sediment samples from Cochiti Reservoir on the Rio Grande.

The primary rationale for this segregation was to test the hypothesis that background radioactivity values from river and reservoir samples are fundamentally different since the grain-size distributions and total organic carbon between river and reservoir samples are different. In addition, the location of one-half of the river sediment stations in close proximity to one another and the Laboratory (i.e., the Group II River stations) may unduly influence the resulting background radioactivity for individual analytes. This situation is somewhat analogous to having five samples from a single Rio Grande station. Finally, portions of the drainage basins for Group I (River) stations do not cross Laboratory lands. However, portions of the drainage basins for Group II (River) stations include Laboratory lands or are near enough that airborne deposition from Laboratory stack and fugitive dust emissions may be of concern (i.e., Frijoles Canyon). 
Initially, general descriptive statistical parameters for all individual and grouped river and reservoir sediment station data were computed. These parameters include the mean (x), median $(\mathrm{m})$, standard deviation (s), range (i.e., the maximum and minimum values), and upper limits for background expressed as $(x+2 s)$ and (BGUL). Definitions for these background expressions are defined below. These values, along with all observed data, are listed in Appendices A and B (the statistical analyses are in the even-numbered Tables A-2 through A-20 and Tables A-21, A-22, and A-23 and in the even-numbered Tables B-2 through B-10 and Tables B-11, B-12, and B-13). Second, the nonparametric Lilliefors goodness-of-fit test (Conover, 1998, pp. 442-447; Lilliefors, 1967, 1969) was applied for each analyte at each station. This test is designed to detect departures from the hypothesized normal distribution, including normally distributed data after a logarithmic or square-root transformation. These transformations are described in Appendix C. Finally, the Lilliefors test was also applied to grouped river and reservoir data. It should be noted that most parametric statistical tests that compare mean radioactivity from different groups are based on the assumption of a normal distribution, whereas nonparametric statistical tests avoid this limitation. Hence, it is important to evaluate individual analyte distributions so that the appropriate statistical test for differences in mean radioactivity can be applied.

The Lilliefors test evaluates the hypothesis that an analyte sample group has a normal distribution (or a normal distribution after a logarithmic or square-root transformation) with an unspecified mean and variance against the alternative that the sample group does not have a normal distribution. This test compares the empirical distribution of the sample group with a normal distribution having the same mean and variance as the sample group. It is similar to the Kolmogoro-Smirnov test, but it adjusts for the fact that the parameters of the normal distribution are estimated from the sample group rather than specified in advance.

Goodness-of-fit statistics from the Lilliefors tests are listed in Table 6 for individual sampling stations for both river and reservoir sediments. According to the Lilliefors statistic at the $95 \%$ significance level, $53.3 \%$ of all analytes from river sediments and $76.0 \%$ of all analytes from reservoir sediments are normally distributed, or are normally distributed after a logarithmic or square-root transformation. This is an important observation. Similar results were previously reported for cesium in soils by Pinder and Smith (1975). However, when the grouped samples are tested, a very different picture emerges. The goodness-of-fit statistics for the grouped river and reservoir data are listed in Table 7. According to the grouped Lilliefors statistic at the $95 \%$ significance level, only $12.2 \%$ of all analytes from river sediments and $38.9 \%$ of all analytes from reservoir sediments are normally distributed or are normally distributed after a logarithmic or square-root transformation. The differences between the individual station (Table 6) and grouped station (Table 7) Lilliefors test results are most likely related to the larger number of samples in the grouped data.

These goodness-of-fit tests suggest that nonparametric statistical tests should be used when comparing mean analyte radioactivity from different groupings. Purtymun et al. (1987) had previously used the nonparametric Kruskal-Wallis test (Conover, 1998, pp. 288-297; Gilbert, 1987, pp. 247-252) for similar analyses; this test was also used here. However, these Lilliefors test results also demonstrate that robust parametric statistical tests may still be used in comparing mean radiochemical information since many of these data are normally distributed (or very nearly normally distributed), as seen in Appendix C. The student's t-test was used for these comparisons since it is the parametric equivalent of the Kruskal-Wallis test.

The univariate statistical tests described above were employed to determine if significant differences in mean radioactivity existed at the various regional river and reservoir sediment stations described above. Each grouped analyte included in the study (i.e., tritium, strontium-90, cesium-137, total uranium, plutonium-238, plutonium-239,-240, americium-241, gross alpha, gross beta, and gross gamma radioactivity) was analyzed separately by comparing the means from Group I to the corresponding means from Group II. Comparisons of individual analyte radioactivity from individual sampling stations were not made because the limited number of samples available for analyses greatly reduces the power of any statistical conclusions. Ideally, the Group I and Group II comparisons should use the parametric student's t-test for both independent and dependent samples. These tests assume a normal distribution within the sample population (or a normal distribution after a logarithmic or square-root transformation). This technique is quite robust, and even large departures from normality often do not significantly alter conclusions. Second, the nonparametric KruskalWallis test for independent samples was used for these same groupings. This test does not require assumptions about the distribution, although distribution shapes are assumed similar. Interpretations of these two tests are similar. Hence, comparisons between grouped stations can be made to see whether differences in mean radioactivity are statistically significant or may be attributed to chance. The student's t-test is more 


\begin{tabular}{|c|c|c|c|c|c|c|c|c|c|c|c|c|c|c|c|c|c|c|c|c|c|c|c|c|c|c|}
\hline \multicolumn{27}{|c|}{ illiefors test for goodness-of-fit at 95\% significance level. Reject the null hypothesis (normal distribution) if Normal, Log, or Sqrt values > Table value. } \\
\hline River & \multicolumn{5}{|c|}{ Rio Chama at Chamita } & \multicolumn{5}{|c|}{ Rio Grande at Embudo } & \multicolumn{5}{|c|}{ Rio Grande at Cochiti Spillway } & \multicolumn{5}{|c|}{ Rio Grande at Bernalillo } & \multicolumn{5}{|c|}{ Jemez River } & \multirow[b]{2}{*}{ Totals } \\
\hline Stations & Samples & Normal & Log & Sqrt & Table & Samples & Normal & Log & Sqrt & Table & Samples & Normal & $\log$ & Sqrt & Table & Samples & Normal & Log & Sqrt & Table & Samples & Normal & Log & Sqrt & Table & \\
\hline${ }^{3} \mathbf{H}$ & 6 & 0.245 & 0.350 & 0.263 & 0.319 & 6 & 0.202 & 0.173 & 0.157 & 0.319 & 1 & & & & & 6 & 0.246 & 0.146 & 0.200 & 0.319 & 5 & 0.251 & 0.272 & 0.220 & 0.337 & 11 \\
\hline${ }^{90} \mathbf{S r}$ & 10 & 0.270 & 0.204 & 0.245 & 0.258 & 11 & 0.325 & 0.217 & 0.281 & 0.249 & 3 & & & & & 10 & 0.283 & 0.177 & 0.238 & 0.258 & 9 & 0.172 & 0.263 & 0.179 & 0.271 & 8 \\
\hline 形 ${ }^{137 s}$ & 23 & 0.172 & 0.243 & 0.125 & 0.180 & 24 & 0.234 & 0.183 & 0.172 & 0.176 & 6 & 0.360 & 0.184 & 0.266 & 0.319 & 23 & 0.094 & 0.245 & 0.155 & 0.180 & 23 & 0.178 & 0.091 & 0.120 & 0.180 & 10 \\
\hline Total U & 19 & 0.150 & 0.116 & 0.125 & 0.195 & 20 & 0.132 & 0.149 & 0.131 & 0.190 & 5 & 0.215 & 0.209 & 0.213 & 0.337 & 20 & 0.139 & 0.136 & 0.118 & 0.190 & 19 & 0.113 & 0.134 & 0.103 & 0.195 & 15 \\
\hline ${ }^{238} \mathbf{P u}$ & 26 & 0.245 & 0.396 & 0.220 & 0.171 & 27 & 0.340 & 0.358 & 0.261 & 0.168 & 8 & 0.344 & 0.483 & 0.422 & 0.285 & 24 & 0.209 & 0.373 & 0.259 & 0.176 & 26 & 0.326 & 0.316 & 0.266 & 0.171 & 0 \\
\hline${ }^{233,244} \mathrm{Pu}$ & 26 & 0.230 & 0.384 & 0.226 & 0.171 & 27 & 0.280 & 0.434 & 0.351 & 0.168 & 8 & 0.313 & 0.471 & 0.396 & 0.285 & 24 & 0.290 & 0.279 & 0.235 & 0.176 & 26 & 0.236 & 0.285 & 0.187 & 0.171 & 0 \\
\hline${ }^{241} \mathrm{Am}$ & 6 & 0.461 & 0.256 & 0.395 & 0.319 & 4 & 0.283 & 0.396 & 0.272 & 0.381 & 1 & & & & & 6 & 0.359 & 0.474 & 0.425 & 0.319 & 5 & 0.273 & 0.404 & 0.242 & 0.337 & 5 \\
\hline G. Alpha & 15 & 0.232 & 0.170 & 0.180 & 0.220 & 15 & 0.158 & 0.153 & 0.157 & 0.220 & 6 & 0.362 & 0.336 & 0.349 & 0.319 & 16 & 0.326 & 0.314 & 0.320 & 0.213 & 16 & 0.141 & 0.144 & 0.143 & 0.213 & 8 \\
\hline G. Beta & 15 & 0.219 & 0.161 & 0.188 & 0.220 & 15 & 0.162 & 0.142 & 0.153 & 0.200 & 6 & 0.451 & 0.442 & 0.447 & 0.319 & 16 & 0.288 & 0.285 & 0.287 & 0.213 & 16 & 0.307 & 0.277 & 0.292 & 0.213 & 6 \\
\hline G. Gamma & 20 & 0.237 & 0.202 & 0.217 & 0.190 & 19 & 0.119 & 0.147 & 0.125 & 0.195 & 4 & 0.285 & 0.288 & 0.288 & 0.381 & 19 & 0.130 & 0.095 & 0.106 & 0.195 & 19 & 0.085 & 0.108 & 0.095 & 0.195 & 12 \\
\hline Total: & & 4 & 5 & 6 & & & 6 & 6 & 7 & & & 2 & 3 & 3 & & & 4 & 4 & 5 & & & 7 & 6 & 7 & & 75 \\
\hline River & \multicolumn{5}{|c|}{ Rio Grande at Otowi } & \multicolumn{5}{|c|}{ Rio Grande below Sandia } & \multicolumn{5}{|c|}{ Rio Grande below Pajarito } & \multicolumn{5}{|c|}{ Rio Grande below Ancho } & \multicolumn{5}{|c|}{ Rio Grande below Frijoles } & \\
\hline Stations & Samples & Normal & $\log$ & Sqrt & Table & Samples & Normal & $\log$ & Sqrt & Table & Samples & Normal & Log & Sqrt & Table & Samples & Normal & Log & Sqrt & Table & Samples & Normal & $\log$ & Sqrt & Table & Totals \\
\hline${ }^{3} \mathbf{H}$ & 6 & 0.212 & 0.376 & 0.291 & 0.319 & 6 & 0.207 & 0.235 & 0.226 & 0.319 & 6 & 0.341 & 0.379 & 0.341 & 0.319 & 5 & 0.167 & 0.172 & 0.168 & 0.337 & 7 & 0.266 & 0.305 & 0.259 & 0.300 & 10 \\
\hline${ }^{90} \mathrm{Sr}$ & 12 & 0.335 & 0.263 & 0.305 & 0.242 & 4 & 0.307 & 0.304 & 0.306 & 0.381 & 4 & 0.391 & 0.276 & 0.315 & 0.381 & 4 & 0.414 & 0.396 & 0.406 & 0.381 & 9 & 0.256 & 0.180 & 0.219 & 0.271 & 8 \\
\hline 初 $\mathrm{Cs}$ & 24 & 0.210 & 0.198 & 0.155 & 0.176 & 16 & 0.147 & 0.235 & 0.140 & 0.213 & 16 & 0.154 & 0.197 & 0.087 & 0.213 & 15 & 0.143 & 0.155 & 0.106 & 0.220 & 20 & 0.143 & 0.334 & 0.225 & 0.190 & 10 \\
\hline Total U & 20 & 0.145 & 0.155 & 0.129 & 0.190 & 14 & 0.400 & 0.317 & 0.360 & 0.227 & 13 & 0.186 & 0.192 & 0.177 & 0.234 & 12 & 0.172 & 0.210 & 0.177 & 0.242 & 16 & 0.083 & 0.159 & 0.117 & 0.213 & 12 \\
\hline${ }^{238} \mathrm{Pu}$ & 27 & 0.214 & 0.334 & 0.200 & 0.168 & 17 & 0.305 & 0.460 & 0.382 & 0.206 & 16 & 0.317 & 0.339 & 0.254 & 0.213 & 17 & 0.215 & 0.315 & 0.173 & 0.206 & 23 & 0.203 & 0.432 & 0.288 & 0.180 & 1 \\
\hline${ }^{233,244} \mathrm{Pu}$ & 27 & 0.347 & 0.208 & 0.294 & 0.168 & 17 & 0.302 & 0.450 & 0.379 & 0.206 & 15 & 0.177 & 0.327 & 0.123 & 0.220 & 17 & 0.253 & 0.270 & 0.178 & 0.206 & 23 & 0.413 & 0.338 & 0.293 & 0.180 & 3 \\
\hline${ }^{241} \mathrm{Am}$ & 5 & 0.277 & 0.394 & 0.290 & 0.337 & 2 & & & & & 2 & & & & & 2 & & & & & 5 & 0.241 & 0.335 & 0.153 & 0.337 & 5 \\
\hline G. Alpha & 16 & 0.203 & 0.138 & 0.168 & 0.213 & 7 & 0.310 & 0.248 & 0.278 & 0.300 & 7 & 0.178 & 0.168 & 0.171 & 0.300 & 7 & 0.142 & 0.161 & 0.151 & 0.300 & 14 & 0.141 & 0.174 & 0.142 & 0.227 & 14 \\
\hline G. Beta & 16 & 0.167 & 0.127 & 0.148 & 0.213 & 7 & 0.305 & 0.251 & 0.267 & 0.300 & 7 & 0.330 & 0.299 & 0.315 & 0.300 & 7 & 0.236 & 0.246 & 0.241 & 0.300 & 13 & 0.205 & 0.225 & 0.216 & 0.234 & 12 \\
\hline G. Gamma & 20 & 0.154 & 0.199 & 0.177 & 0.190 & 6 & 0.253 & 0.349 & 0.301 & 0.319 & 6 & 0.346 & 0.363 & 0.356 & 0.319 & 6 & 0.167 & 0.194 & 0.182 & 0.319 & 15 & 0.190 & 0.146 & 0.157 & 0.220 & 10 \\
\hline Total: & & 6 & 3 & 7 & & & 4 & 4 & 6 & & & 4 & 5 & 5 & & & 6 & 6 & 8 & & & 8 & 6 & 7 & & 85 \\
\hline Reservoir & \multicolumn{5}{|c|}{ Abiquiu Reservoir } & \multicolumn{5}{|c|}{ El Vado Reservoir } & \multicolumn{5}{|c|}{ Heron Reservoir } & \multicolumn{5}{|c|}{ Rio Grande Reservoir } & & Cochit & i Reserv & voir & & \\
\hline Stations & Samples & Normal & $\log$ & Sqrt & Table & Samples & Normal & $\log$ & Sqrt & Table & Samples & Normal & Log & Sqrt & Table & Samples & Normal & Log & Sqrt & Table & Samples & Normal & $\log$ & Sqrt & Table & Totals \\
\hline${ }^{3} \mathrm{H}$ & 23 & 0.145 & 0.205 & 0.133 & 0.180 & 6 & 0.185 & 0.234 & 0.184 & 0.319 & 8 & 0.266 & 0.228 & 0.216 & 0.285 & 6 & 0.189 & 0.312 & 0.239 & 0.319 & 24 & 0.196 & 0.173 & 0.150 & 0.176 & 13 \\
\hline 量 $\mathrm{Sr}$ & 29 & 0.152 & 0.211 & 0.149 & 0.163 & 6 & 0.277 & 0.311 & 0.293 & 0.319 & 9 & 0.320 & 0.218 & 0.258 & 0.271 & 3 & & & & & 24 & 0.269 & 0.147 & 0.211 & 0.176 & 8 \\
\hline${ }^{137} \mathrm{Cs}$ & 32 & 0.201 & 0.151 & 0.138 & 0.157 & 12 & 0.224 & 0.122 & 0.171 & 0.242 & 15 & 0.205 & 0.116 & 0.152 & 0.220 & 9 & 0.148 & 0.220 & 0.171 & 0.271 & 33 & 0.128 & 0.127 & 0.082 & 0.154 & 14 \\
\hline Total U & 32 & 0.120 & 0.130 & 0.121 & 0.157 & 12 & 0.224 & 0.172 & 0.202 & 0.242 & 15 & 0.208 & 0.116 & 0.161 & 0.220 & 9 & 0.230 & 0.245 & 0.242 & 0.271 & 30 & 0.133 & 0.182 & 0.149 & 0.162 & 14 \\
\hline${ }^{238} \mathrm{Pu}$ & 40 & 0.218 & 0.132 & 0.155 & 0.140 & 15 & 0.219 & 0.189 & 0.149 & 0.220 & 18 & 0.293 & 0.194 & 0.253 & 0.200 & 10 & 0.352 & 0.254 & 0.300 & 0.258 & 45 & 0.300 & 0.231 & 0.263 & 0.132 & 6 \\
\hline${ }^{23,240} \mathbf{P u}$ & 40 & 0.080 & 0.093 & 0.087 & 0.140 & 15 & 0.176 & 0.216 & 0.196 & 0.220 & 18 & 0.191 & 0.167 & 0.179 & 0.200 & 10 & 0.226 & 0.207 & 0.216 & 0.258 & 45 & 0.240 & 0.223 & 0.231 & 0.132 & 12 \\
\hline${ }^{241} \mathrm{Am}$ & 17 & 0.209 & 0.430 & 0.303 & 0.206 & 9 & 0.166 & 0.352 & 0.208 & 0.271 & 12 & 0.118 & 0.293 & 0.169 & 0.242 & 9 & 0.174 & 0.411 & 0.218 & 0.271 & 21 & 0.240 & 0.170 & 0.150 & 0.187 & 8 \\
\hline G. Alpha & 17 & 0.187 & 0.181 & 0.185 & 0.206 & 9 & 0.1 & 0.154 & 0.154 & 0.271 & 12 & 0.193 & 0.183 & 0.186 & 0.242 & 9 & 0.262 & 0.240 & 0.251 & 0.271 & 18 & 0.324 & 0.307 & 0.316 & 0.200 & 12 \\
\hline G. Beta & 17 & 0.159 & 0.213 & 0.185 & 0.206 & 9 & 0.165 & 0.163 & 0.165 & 0.271 & 12 & 0.215 & 0.152 & 0.184 & 0.242 & 9 & 0.140 & 0.171 & 0.143 & 0.271 & 18 & 0.133 & 0.120 & 0.114 & 0.200 & 14 \\
\hline G. Gamma & 26 & 0.086 & 0.140 & 0.112 & 0.171 & 9 & 0.253 & 0.196 & 0.200 & 0.271 & 12 & 0.188 & 0.252 & 0.224 & 0.242 & 9 & 0.232 & 0.251 & 0.229 & 0.271 & 24 & 0.142 & 0.203 & 0.174 & 0.176 & 13 \\
\hline Total: & & 7 & 6 & 8 & & & 10 & 9 & 10 & & & 8 & 8 & 9 & & & 8 & 8 & 8 & & & 4 & 5 & 6 & & 114 \\
\hline
\end{tabular}


Table 7. Computed Lilliefors Statistic to Test for Normal, Lognormal, and Square-Root Distributions at Grouped Sampling Stations.

\begin{tabular}{|c|c|c|c|c|c|c|c|c|c|c|c|c|c|c|c|c|}
\hline \multicolumn{17}{|c|}{ Lilliefors test for goodness-of-fit at $95 \%$ significance level. Reject the null hypothesis (normal distribution) if Normal, Log, or Sqrt values > Table value. } \\
\hline River & \multicolumn{5}{|c|}{ Combined River Sediments } & \multicolumn{5}{|c|}{ Group I River Sediments } & \multicolumn{5}{|c|}{ Group II River Sediments } & \multirow[b]{2}{*}{ Total } \\
\hline Analytes & Samples & Normal & $\log$ & Sqrt & Table & Samples & Normal & $\log$ & Sqrt & Table & Samples & Normal & Log & Sqrt & Table & \\
\hline${ }^{3} \mathbf{H}$ & 54 & 0.141 & 0.192 & 0.130 & 0.121 & 24 & 0.223 & 0.152 & 0.181 & 0.176 & 30 & 0.161 & 0.237 & 0.175 & 0.161 & 1 \\
\hline${ }^{90} \mathrm{Sr}$ & 76 & 0.307 & 0.196 & 0.256 & 0.102 & 43 & 0.250 & 0.133 & 0.187 & 0.135 & 33 & 0.344 & 0.259 & 0.307 & 0.154 & 1 \\
\hline${ }^{137} \mathrm{Cs}$ & 190 & 0.128 & 0.121 & 0.085 & 0.064 & 99 & 0.146 & 0.073 & 0.098 & 0.089 & 91 & 0.113 & 0.186 & 0.114 & 0.093 & 1 \\
\hline Total U & 158 & 0.096 & 0.089 & 0.078 & 0.071 & 83 & 0.098 & 0.095 & 0.078 & 0.097 & 75 & 0.136 & 0.121 & 0.114 & 0.102 & 2 \\
\hline${ }^{238} \mathrm{Pu}$ & 211 & 0.237 & 0.368 & 0.257 & 0.061 & 111 & 0.241 & 0.432 & 0.301 & 0.084 & 100 & 0.237 & 0.327 & 0.230 & 0.089 & 0 \\
\hline${ }^{239,240} \mathrm{Pu}$ & 210 & 0.294 & 0.358 & 0.267 & 0.061 & 111 & 0.230 & 0.424 & 0.290 & 0.084 & 99 & 0.310 & 0.287 & 0.279 & 0.089 & 0 \\
\hline${ }^{241} \mathrm{Am}$ & 38 & 0.410 & 0.327 & 0.313 & 0.144 & 22 & 0.459 & 0.312 & 0.363 & 0.183 & 16 & 0.212 & 0.395 & 0.229 & 0.213 & 1 \\
\hline G. Alpha & 119 & 0.234 & 0.199 & 0.217 & 0.081 & 68 & 0.221 & 0.201 & 0.209 & 0.107 & 51 & 0.137 & 0.109 & 0.110 & 0.124 & 2 \\
\hline G. Beta & 118 & 0.212 & 0.163 & 0.188 & 0.082 & 68 & 0.231 & 0.215 & 0.223 & 0.107 & 50 & 0.220 & 0.165 & 0.192 & 0.125 & 0 \\
\hline G. Gamma & 134 & 0.135 & 0.109 & 0.120 & 0.077 & 81 & 0.112 & 0.077 & 0.094 & 0.098 & 53 & 0.159 & 0.217 & 0.186 & 0.122 & 2 \\
\hline Total: & & 0 & 0 & 0 & & & 0 & 5 & 2 & & & 1 & 1 & 1 & & 10 \\
\hline Reservoir & \multicolumn{5}{|c|}{ Combined Reservoir Sediments } & \multicolumn{5}{|c|}{ Group I Reservoir Sediments } & \multicolumn{5}{|c|}{ Group II Reservoir Sediments } & \\
\hline Analytes & Samples & Normal & Log & Sqrt & Table & Samples & Normal & $\log$ & Sqrt & Table & Samples & Normal & $\log$ & Sqrt & Table & Total \\
\hline${ }^{3} \mathbf{H}$ & 67 & 0.173 & 0.147 & 0.127 & 0.108 & 43 & 0.165 & 0.160 & 0.120 & 0.135 & 24 & 0.196 & 0.173 & 0.150 & 0.176 & 3 \\
\hline${ }^{90} \mathrm{Sr}$ & 71 & 0.195 & 0.128 & 0.154 & 0.105 & 47 & 0.175 & 0.216 & 0.152 & 0.129 & 24 & 0.269 & 0.147 & 0.211 & 0.176 & 1 \\
\hline${ }^{137} \mathrm{Cs}$ & 101 & 0.120 & 0.070 & 0.065 & 0.088 & 68 & 0.146 & 0.076 & 0.087 & 0.107 & 33 & 0.128 & 0.127 & 0.082 & 0.154 & 7 \\
\hline Total U & 98 & 0.055 & 0.117 & 0.083 & 0.090 & 68 & 0.057 & 0.109 & 0.070 & 0.107 & 30 & 0.133 & 0.182 & 0.149 & 0.161 & 6 \\
\hline${ }^{238} \mathrm{Pu}$ & 128 & 0.292 & 0.199 & 0.242 & 0.078 & 83 & 0.191 & 0.099 & 0.148 & 0.097 & 45 & 0.300 & 0.231 & 0.263 & 0.132 & 0 \\
\hline${ }^{239,240} \mathrm{Pu}$ & 128 & 0.221 & 0.193 & 0.207 & 0.078 & 83 & 0.137 & 0.108 & 0.123 & 0.097 & 45 & 0.240 & 0.223 & 0.231 & 0.132 & 0 \\
\hline${ }^{241} \mathbf{A m}$ & 68 & 0.233 & 0.165 & 0.148 & 0.107 & 47 & 0.106 & 0.261 & 0.108 & 0.129 & 21 & 0.240 & 0.170 & 0.150 & 0.187 & 4 \\
\hline G. Alpha & 65 & 0.234 & 0.210 & 0.222 & 0.110 & 47 & 0.119 & 0.099 & 0.100 & 0.129 & 18 & 0.324 & 0.307 & 0.316 & 0.200 & 3 \\
\hline G. Beta & 65 & 0.124 & 0.081 & 0.103 & 0.110 & 47 & 0.113 & 0.100 & 0.093 & 0.129 & 18 & 0.133 & 0.120 & 0.114 & 0.200 & 8 \\
\hline G. Gamma & 80 & 0.096 & 0.135 & 0.106 & 0.099 & 56 & 0.125 & 0.195 & 0.158 & 0.118 & 24 & 0.142 & 0.203 & 0.174 & 0.176 & 3 \\
\hline Total: & & 2 & 2 & 3 & & & 4 & 3 & 6 & & & 4 & 5 & 6 & & 35 \\
\hline
\end{tabular}


robust than its nonparametric counterpart. However, the Kruskal-Wallis test is generally regarded by many statisticians as more useful since questions about the distribution may be avoided (the assumption about shape is usually relaxed since most environmental data are right-skewed). In short, the Kruskal-Wallis test is both parameter and distribution free, but the results are not as robust. Results from these tests are summarized below.

\section{River Sediments}

When the mean radiochemical data for individual analytes in the Group I (River) sediments were compared with the corresponding means from the Group II (River) sediments, several differences were detected as seen in Table A-24. According to the student's t-test for independent samples with unequal variance at the $95 \%$ significance level, Group II sediments have a higher mean concentration for total uranium ( 2.44 versus $2.09 \mathrm{mg} / \mathrm{kg}$ ). However, Group I sediments have higher mean radioactivity for gross alpha ( 4.9 versus $3.3 \mathrm{pCi} / \mathrm{g}$ ). There were no significant differences between Group I and Group II mean sediment radioactivity for tritium $(0.08$ versus $0.03 \mathrm{nCi} / \mathrm{l})$, strontium- $90(0.17$ versus $0.28 \mathrm{pCi} / \mathrm{g})$, cesium-137 (0.15 versus $0.14 \mathrm{pCi} / \mathrm{g})$, plutonium-238 (0.001 versus $0.001 \mathrm{pCi} / \mathrm{g})$, plutonium-239,-240 $(0.003$ versus $0.005 \mathrm{pCi} / \mathrm{g})$, americium -241 ( 0.006 versus $0.003 \mathrm{pCi} / \mathrm{g})$, gross beta ( 4.0 versus $3.6 \mathrm{pCi} / \mathrm{g})$, and gross gamma (2.6 versus $2.3 \mathrm{pCi} / \mathrm{g}$ ) radioactivity.

A significant difference in mean total uranium concentration on soils and sediments is related to differences in areal geology (Longmire et al., 1996; Fresquez et al., 1996). A significantly larger mean gross alpha level for Group I (River) sediments compared with Group II (River) samples is difficult to explain since most of the alpha emitters (e.g., uranium and plutonium isotopes) are slightly higher for Group II (River). The only exception to this observation is americium, which has a somewhat higher mean radioactivity for Group I (River) sediments.

Nearly identical results were obtained when the more robust student's t-test for independent samples with equal variance was applied (Table A-25). Theoretically, this test is more robust than the first test because it has more degrees of freedom; however, individual analyte variances from each group are not always equal. Differences between the tests that result from the assumption of equal or unequal variances are small and probably result from the large number of samples in the analysis. The student's t-test for dependent (paired) samples was not run because the pairing requirement dramatically reduced the total number of samples available for analysis.

It is important to point out the t-statistic results for plutonium-239,-240 listed in Tables A-24 and A-25. Both of the t-test results indicate that the mean radioactivity for plutonium-239,-240 are not significantly different between Group I (River) and Group II (River) samples. However, if these tests are repeated at the $90 \%$ significance level, then the mean radioactivity for plutonium-239,-240 is higher for Group II (River) sediments than for Group I (River) sediments. This is an important observation that is confirmed below.

According to the nonparametric Kruskal-Wallis test for independent samples at the $95 \%$ significance level (Table A-26), Group II (River) sediments have a higher mean radioactivity for total uranium and plutonium-239,-240 compared with Group I (River) means. No other differences in mean radioactivity were detected, including a difference for the gross alpha level as in the t-tests presented above. These results suggest that the student's t-test for gross alpha may yield a false positive (i.e., that no mean difference for gross alpha actually exists). As mentioned above, significant differences in total uranium concentrations on soils and sediments are related to differences in areal geology (Longmier et al., 1996; Fresquez et al., 1996). Differences in plutonium-239,-240 radioactivity between Group I (River) and Group II (River) sediments are most likely related to both fallout sources and historical Laboratory releases into the environment (Stoker et al., 1981; Graf, 1996). Hence, the rationale for segregating river samples into separate upstream and downstream groups appears justified. In other words, only sediment samples from the Group I (River) stations should be used to establish background since Group II (River) samples may be receiving trace levels of plutonium-239,-240 from both fallout and Laboratory sources.

Despite the statistical test results presented above, it is apparent that the difference in mean plutonium239,-240 radioactivity levels between Group I (River) and Group II (River) sediments is very small. Furthermore, this difference $(0.002 \mathrm{pCi} / \mathrm{g})$ is less than the detection limit $(0.005 \mathrm{pCi} / \mathrm{g})$ for 
plutonium-239,-240. Hence, there is some added uncertainty in this difference. In other words, these mean differences may be attributable to the laboratory detection limit for plutonium. However, it is still prudent to use only sediment samples from Group I (River) stations to establish background radioactivity levels.

\section{Reservoir Sediments}

When the mean radiochemical data for the Group I (Reservoir) sediments were compared with the corresponding means from the Group II (Reservoir) sediments, several differences were detected as seen in Table B-14. According to the student's t-test for independent samples with unequal variance at the $95 \%$ significance level, Group II (Reservoir) sediments have a higher mean radioactivity for plutonium-238 ( 1.5 versus $0.4 \mathrm{fCi} / \mathrm{g})$, plutonium-239,-240 (19.1 versus $7.7 \mathrm{fCi} / \mathrm{g})$, and americium-241 (0.011 versus $0.004 \mathrm{pCi} / \mathrm{g}$ ). There were no significant differences between Group I (Reservoir) and Group II (Reservoir) mean sediment radioactivity for tritium $(0.04$ versus $0.02 \mathrm{nCi} / \mathrm{l})$, strontium- $90(0.21$ versus $0.32 \mathrm{pCi} / \mathrm{g})$, cesium-137 (0.31 versus $0.38 \mathrm{pCi} / \mathrm{g})$, total uranium (3.01 versus $3.27 \mathrm{mg} / \mathrm{kg})$, gross alpha $(8.0$ versus $13.6 \mathrm{pCi} / \mathrm{g})$, gross beta $(5.5$ versus $6.7 \mathrm{pCi} / \mathrm{g})$, and gross gamma $(2.3$ versus $2.8 \mathrm{pCi} / \mathrm{g}$ ) radioactivity.

Nearly identical results were obtained when the more robust student's t-test for independent samples with equal variance was applied (Table B-15). According to the student's t-test for independent samples with equal variance at the 95\% significance level, Group II (Reservoir) sediments have a higher mean radioactivity for plutonium-238, plutonium-239,-240, americium-241, and gross alpha radioactivity. There were no other significant differences between Group I (Reservoir) and Group II (Reservoir) sediments. The student's t-test for dependent (paired) samples was not run because the pairing requirement dramatically reduced the total number of samples available for analysis.

According to the nonparametric Kruskal-Wallis test for independent samples at the 95\% significance level (Table B-16), Group II (Reservoir) sediments have a higher mean radioactivity than Group I (Reservoir) sediments for cesium-137, plutonium-238, plutonium-239,-240, americium-241, and gross gamma. No other differences in mean radioactivity were detected. All of these differences are most likely related to both fallout sources and historical Laboratory releases into the environment (Stoker et al., 1981; Graf, 1996). Hence, the rationale for segregating reservoir samples into separate upstream and downstream groups appears justified.

\section{Comparison of River and Reservoir Sediments}

When the mean radiochemical data for the Group I (River) sediments were compared with the corresponding means from the Group I (Reservoir) sediments, several differences were detected as seen in Table 8. According to the student's t-test for independent samples with unequal variance at the $95 \%$ significance level (Table 8a), Group I (Reservoir) sediments have a higher mean radioactivity for cesium-137 (0.31 versus $0.15 \mathrm{pCi} / \mathrm{g}$ ), total uranium (3.01 versus $2.09 \mathrm{mg} / \mathrm{kg}$ ), plutonium-239,-240 ( 7.74 versus $3.05 \mathrm{fCi} / \mathrm{g})$, gross alpha $(8.0$ versus $4.9 \mathrm{pCi} / \mathrm{g})$, and gross beta $(5.5$ versus $4.0 \mathrm{pCi} / \mathrm{g})$ radioactivity. However, Group I (River) sediments have higher mean radioactivity for plutonium-238 (1.07 versus $0.40 \mathrm{fCi} / \mathrm{g}$ ). There were no significant differences between Group I (River) and Group I (Reservoir) mean sediment radioactivity for tritium $(0.09$ versus $0.04 \mathrm{nCi} / 1)$, strontium- $90(0.17$ versus $0.21 \mathrm{pCi} / \mathrm{g}$ ), americium-241 ( 0.006 versus $0.004 \mathrm{pCi} / \mathrm{g}$ ), and gross gamma (2.6 versus $2.3 \mathrm{pCi} / \mathrm{g}$ ) radioactivity.

The significant difference in mean plutonium levels between Group I (River) and Group I (Reservoir) samples may be related to the laboratory detection limits for plutonium $(5.0$ and $0.1 \mathrm{fCi} / \mathrm{g}$ for river and reservoir sediments, respectively). Significantly higher mean radioactivity for cesium-137, total uranium, plutonium-239,-240, gross alpha, and gross beta radioactivity were also found in Group I (Reservoir) sediments compared with Group I (River) sediments. This situation is probably related to the smaller grain size distributions and to the higher organic contents that are typically found in reservoir sediments.

Nearly identical results were obtained when the more robust student's t-test for independent samples with equal variance was applied (Table $8 b$ ). Note, however, that plutonium-238 is no longer significantly different between the two groups. Theoretically, this test is more robust than the first test because it has more degrees of freedom; however, individual analyte variances from each group are not always equal. The student's t-test for dependent (paired) samples was not run because the pairing requirement dramatically reduced the total number of samples available for analysis. 
According to the nonparametric Kruskal-Wallis test for independent samples at the $95 \%$ significance level (Table 8c), similar results were obtained compared with the t-test results presented above. However, there are some differences. Thus, Group I (Reservoir) sediments have a higher mean radioactivity for stronium-90, cesium-137, total uranium, plutonium-239,-240, gross alpha, and gross beta radioactivity compared with Group I (River) means. In addition, Group I (River) sediments had higher mean radioactivity for americium-241. No other differences in mean radioactivity were detected, including any differences for tritium, plutonium-238, and gross gamma radioactivity.

These tests confirm the hypothesis that background river and reservoir sediment samples are significantly different from one another. Hence, different background levels for individual radionuclides should be computed for river and reservoir data. In fact, according to our analyses, background reservoir sediments have significantly higher levels for stronium-90, cesium-137, total uranium, plutonium-239,-240, gross alpha, and gross beta radioactivity than do background river sediments. This is an important new finding. These differences are probably due to the smaller grain size distributions and to the higher organic contents that are typically found in reservoir sediments.

\section{CALCULATION OF BACKGROUND VALUES}

For the purposes of this study, the background radioactivity for a particular analyte can be defined in several different ways. First, it can be approximated by the mean radioactivity computed from the observed annual series for that radionuclide. Furthermore, the upper limit of this background level can be defined as this mean radioactivity plus two times the standard deviation as found from the annual series. This definition requires either a normal distribution for the observed data or a data transformation that results in a normal distribution. This transformation requirement may be computationally inconvenient, or the assumptions for normality may not always be completely satisfied. Hence, a second approximation for background radioactivity can be defined as the observed median (or the 0.50 quantile from an unknown distribution). The upper limit of this second background value is defined as the radioactivity corresponding to the 0.95 quantile (Gilbert, 1987). This second definition does not require any particular statistical distribution. Historically, the first definition has been used by the Laboratory to define the upper limit for radioisotope background values. The second definition was adopted in this study because radionuclide distributions do not always fit a normal, lognormal, or square-root transformed pattern (or other appropriate data transformation). Both definitions are used in this study to report background values for specific radionuclides in sediments. Obviously, other definitions could have been used to define the upper limit of background values for radiochemicals on sediments. However, there are no recommended standards or published guidelines that specify how to compute these background values. Our definitions are simply based on accepted measures of central tendency and the extent of natural variability in typical environmental data sets. In addition, our definitions have the advantage of computational ease.

It should be noted that at routine surveillance program detection limits and observed fallout levels for a given radionuclide in river and reservoir sediments, one cannot routinely distinguish between levels from Laboratory-derived radionuclide sources and naturally occurring fallout. In this study, we simply assume that if a particular radioactivity value from a river or reservoir sediment sample exceeds the computed upper limit background value, then it probably came from a Laboratory effluent source. If the observed radioactivity is less than the computed upper limit for background, we likewise assume that it originated from fallout (or natural sources in the case of total uranium or tritium). This assumption is realistic because it is based on statistical theory developed for normally distributed data where the first definition for background is used (i.e., mean plus two times standard deviation). It is also realistic when the nonparametric definition for background is employed (i.e., 0.95 quantile). At the $95 \%$ significance level, both of these theoretical approaches predict that approximately one observed background radionuclide value in twenty may exceed the computed upper limit for background and still be from fallout. Radioactivity values in background sediments from individual stations near Los Alamos tend to have a normal, lognormal, or square-roottransformed distribution. However, significant departures from normality are apparent in much of the grouped data as seen in Table 7 and Appendix C. As previously discussed, these differences are probably due to the larger number of samples in the grouped data. Finally, the two alternative definitions for the upper limit for background radioactivity are conservative in that they probably underestimate the actual upper limit. 


\section{River Sediments}

Historical river sediment data from the river stations are tabulated in Appendix A (odd numbered Tables A-1 through A-19), including statistical summaries for each river station (even numbered Tables A-2 through A-20). Table 9 summarizes background statistical information from radiochemical analyses for Group I river sediments (i.e., from Chamita, Embudo, Cochiti Spillway, Bernalillo, and Jemez stations). Data from Otowi, Sandia, Pajarito, Ancho, and Frijoles stations were not included in this background summary because of the possibility of upstream contaminant releases from Laboratory lands.

\section{Reservoir Sediments}

Historical reservoir sediment data from the reservoir stations are tabulated in Appendix B (odd numbered Tables B-1 through B-9), including statistical summaries for each reservoir (even numbered Tables B-2 through B-10). Table 10 summarizes statistical information from the radionuclide analyses for Group I (Reservoir) sediments, including an upper limit for background radioactivity of each analyte.

\section{Comparisons of Background Values with Other Published Results}

The upper limit for background values for river and reservoir sediments are compared with other published background values in Table 11. In making such comparisons, however, we must be aware of important differences in the definition for background values. In this report, the upper limit for background radioactivity was defined two different ways. In the first definition, it was set equal to the sample mean $(\mathrm{x})$ plus two times the sample standard deviation (s) (or $x+2 s$ in Table 11). In the second definition, it was set equal to the 0.95 (two-tail) quantile (or BGUL in Table 11). However, the Laboratory's ER Project refers to the upper limit of background as the upper tolerance limit (or ER UTL in Table 11). Furthermore, the ER Project actually defines their UTL four different ways using the 95\% confidence level for each approach (Ryti et al., 1998, pp. 3 and 4). Three of these methods are based on the normal distribution (i.e., for normally distributed data with no data transformation, for normally distributed data after a lognormal transformation, and for normally distributed data after a square-root data transformation). One disadvantage associated with the data transformations used for the ER background values is that any negative radionuclide values may be automatically truncated from the calculations, since these negative transformations are not defined (i.e., log transformation) or are imaginary (i.e., the square-root transformation). Hence, the reported upper limits for background may be unintentionally skewed toward the higher values. The fourth ER approach is based on nonparametic order statistics and quantiles; it is computationally very similar to our BGUL. The ER definition, however, sets the upper limit of background equal to the upper $95 \%$ confidence interval (two-tail) on the 0.95 quantile. Finally, the upper limit for background radioactivity has been historically defined (Purtymun et al., 1987) as the mean plus two times the standard deviation (or Historical BG in Table 11).

All of the background definitions used in Table 11 will theoretically yield similar results if the data are normally distributed and if there are a large number of samples. In practice, however, these data comparisons tend to produce noticeable differences in background values for a number of reasons: (1) a different number of samples are used to compute background values; (2) different laboratories analyzed different samples collected from different locations and at different times; and (3) different limits of quantification were specified for individual analytes over time or between different analytical laboratories. Finally, it should be noted that even if all of these factors were the same, then individual analyte ER UTL values would still be slightly higher than our BGUL values because of differences in the definitions for these terms.

Except for tritium, total uranium, and plutonium, all of the values in Table 11 are quite close to one another. In general, the ER values resemble our river BGUL sediment values since the ER samples were collected from canyon bottoms within and near the Laboratory boundary. Tritium analyses for the ER samples have a somewhat lower detection limit (i.e., $0.500 \mathrm{nCi} / \mathrm{l}$ ) compared with the Environmental Surveillance Program detection limit (i.e., $0.700 \mathrm{nCi} / \mathrm{l})$. By comparison, tritium in rainwater near Pajarito Plateau varies between 0.020 and $0.450 \mathrm{nCi} / 1$ (Adams et al., 1995), while tritium in shallow groundwater varies between 0.016 and $0.065 \mathrm{nCi} / 1$ (Blake et al., 1995). These observations suggest that a lower detection limit for tritium could be used to identify surface recharge to the regional aquifer below Pajarito Plateau. Such a program is currently being used by the Laboratory (Rogers, 1998); however, these sample analyses are 
Table 9. Statistical Summary for Group I River Sediment Background Stations. ${ }^{\text {a }}$ The Sampling Period Runs from 1974 through 1997.

\begin{tabular}{|c|c|c|c|c|c|c|c|c|c|}
\hline Analyte & Units & $\operatorname{Mean}(\mathbf{x})$ & Median (m) & $\operatorname{Std} \operatorname{Dev}(s)^{b}$ & No. of Samples & Min Value ${ }^{c}$ & Max Value ${ }^{d}$ & BG $(x+2 s)^{e}$ & BGUL $^{f}$ \\
\hline${ }^{3} \mathrm{H}$ & $\mathrm{nCi} / 1$ & 0.09 & 0.00 & 0.41 & 24 & -0.60 & 1.00 & 0.91 & 1.00 \\
\hline${ }^{90} \mathrm{Sr}$ & $\mathrm{pCi} / \mathrm{g}$ & 0.19 & 0.10 & 0.28 & 44 & -0.15 & 1.20 & 0.76 & 1.02 \\
\hline${ }^{137} \mathrm{Cs}$ & $\mathrm{pCi} / \mathrm{g}$ & 0.15 & 0.12 & 0.14 & 99 & -0.08 & 0.82 & 0.43 & 0.56 \\
\hline Total U & $\mathrm{mg} / \mathrm{kg}$ & 2.09 & 1.90 & 0.95 & 83 & 0.30 & 5.80 & 4.00 & 4.49 \\
\hline${ }^{238} \mathrm{Pu}$ & $\mathrm{pCi} / \mathrm{g}$ & 0.001 & 0.001 & 0.003 & 111 & -0.02 & 0.011 & 0.007 & 0.009 \\
\hline $239,240 \mathrm{Pu}$ & $\mathrm{pCi} / \mathrm{g}$ & 0.003 & 0.002 & 0.005 & 111 & -0.03 & 0.032 & 0.013 & 0.013 \\
\hline${ }^{241} \mathrm{Am}$ & $\mathrm{pCi} / \mathrm{g}$ & 0.006 & 0.003 & 0.017 & 22 & -0.003 & 0.080 & 0.039 & 0.076 \\
\hline Gross Alpha & $\mathrm{pCi} / \mathrm{g}$ & 4.9 & 3.0 & 4.8 & 68 & 0.5 & 25.0 & 14.5 & 15.7 \\
\hline Gross Beta & $\mathrm{pCi} / \mathrm{g}$ & 4.0 & 2.9 & 3.9 & 68 & 0.5 & 19.0 & 11.7 & 17.6 \\
\hline Gross Gamma & $\mathrm{pCi} / \mathrm{g}$ & 2.6 & 2.2 & 2.7 & 81 & -4.1 & 11.0 & 8.1 & 8.8 \\
\hline
\end{tabular}

a Group I stations include Rio Chama at Chamita; Rio Grande at Embudo, Cochiti Spillway, and Bernalillo; and Jemez River.

b Standard deviation.

${ }^{c}$ Minimum value.

d Maximum value.

e Upper limit for background $(x+2 s)=$ mean plus two times standard deviation (historical comparisons); computed values subject to round-off error.

${ }^{\mathrm{f}}$ Upper limit for background $=0.95$ (two-tail) quantile (recommended). 
Table 10. Statistical Summary from Group I Reservoir Sediment Background Stations. ${ }^{a}$

The Sampling Period Runs from 1979 through 1997.

\begin{tabular}{|c|c|c|c|c|c|c|c|c|c|}
\hline Analyte & Units & Mean (x) & Median (m) & Std Dev $(s)^{b}$ & No. of Samples & Min Value ${ }^{c}$ & Max Value & $\mathrm{BG}(\mathrm{x}+2 \mathrm{~s})^{\mathrm{e}}$ & BGUL $^{f}$ \\
\hline${ }^{3} \mathrm{H}$ & $\mathrm{nCi} / 1$ & 0.04 & 0.00 & 0.25 & 43 & -0.50 & 0.60 & 0.53 & 0.54 \\
\hline${ }^{90} \mathrm{Sr}$ & $\mathrm{pCi} / \mathrm{g}$ & 0.21 & 0.20 & 0.21 & 47 & -0.21 & 0.80 & 0.63 & 1.19 \\
\hline${ }^{137} \mathrm{Cs}$ & $\mathrm{pCi} / \mathrm{g}$ & 0.31 & 0.25 & 0.22 & 68 & 0.00 & 1.10 & 0.75 & 0.98 \\
\hline Total U & $\mathrm{mg} / \mathrm{kg}$ & 3.01 & 3.04 & 0.75 & 68 & 1.32 & 5.30 & 4.50 & 4.58 \\
\hline${ }^{238} \mathrm{Pu}$ & $\mathrm{fCi} / \mathrm{g}$ & 0.4 & 0.3 & 0.3 & 83 & 0.0 & 2.2 & 1.1 & 1.2 \\
\hline${ }^{239,240} \mathrm{Pu}$ & $\mathrm{fCi} / \mathrm{g}$ & 7.7 & 6.7 & 6.0 & 83 & 0.2 & 38.8 & 19.8 & 20.1 \\
\hline${ }^{241} \mathrm{Am}$ & $\mathrm{pCi} / \mathrm{g}$ & 0.004 & 0.004 & 0.003 & 47 & -0.001 & 0.011 & 0.009 & 0.010 \\
\hline Gross Alpha & $\mathrm{pCi} / \mathrm{g}$ & 8.0 & 7.6 & 3.3 & 47 & 2.0 & 16.4 & 14.6 & 15.9 \\
\hline Gross Beta & $\mathrm{pCi} / \mathrm{g}$ & 5.5 & 5.5 & 2.1 & 47 & 0.9 & 11.7 & 9.7 & 9.7 \\
\hline Gross Gamma & $\mathrm{pCi} / \mathrm{g}$ & 2.3 & 2.4 & 1.0 & 56 & -1.3 & 4.8 & 4.4 & 3.6 \\
\hline
\end{tabular}

${ }^{a}$ Group I stations include the upper, middle, and lower stations in Abiquiu, El Vado, and Heron Reservoirs on the Rio Chama and Rio Grande Reservoir on the Rio Grande.

b Standard deviation.

c Minimum value.

d Maximum value.

e Upper limit for background $(x+2 s)=$ mean plus two times standard deviation (historical comparisons); computed values subject to round-off error.

${ }^{\mathrm{f}}$ Upper limit for background $=0.95$ (two-tail) quantile (recommended). 


\section{Table 11. Comparison of Reported Background Radioactivity in Sediments.}

\begin{tabular}{|c|c|c|c|c|c|c|c|c|}
\hline Analyte & Units & River BG $(x+2 s)^{a}$ & River BGUL ${ }^{b}$ & Reservoir BG $(x+2 s)^{a}$ & Reservoir BGUL ${ }^{\mathbf{b}}$ & ER UTL ${ }^{c}$ & ER UTL $^{d}$ & Historical $\mathbf{B G}^{\mathbf{e}}$ \\
\hline${ }^{3} \mathrm{H}$ & $\mathrm{nCi} / \mathrm{l}$ & 0.91 & 1.00 & 0.53 & 0.54 & --- & 0.77 & --- \\
\hline${ }^{90} \mathrm{Sr}$ & $\mathrm{pCi} / \mathrm{g}$ & 0.76 & 1.02 & 0.93 & 1.19 & 1.04 & 1.31 & 0.87 \\
\hline${ }^{137} \mathrm{Cs}$ & $\mathrm{pCi} / \mathrm{g}$ & 0.43 & 0.56 & 0.75 & 0.98 & 0.90 & 1.65 & 0.44 \\
\hline Total U & $\mathrm{mg} / \mathrm{kg}$ & 4.00 & 4.49 & 4.50 & 4.58 & 6.99 & --- & 4.40 \\
\hline${ }^{238} \mathrm{Pu}$ & $\mathrm{fCi} / \mathrm{g}$ & 7.0 & 8.7 & 1.1 & 1.2 & 6.0 & 23.0 & 6.0 \\
\hline $239,240 \mathrm{Pu}$ & $\mathrm{fCi} / \mathrm{g}$ & 13.0 & 13.0 & 19.8 & 20.1 & 68.0 & 54.0 & 23.0 \\
\hline${ }^{241} \mathrm{Am}$ & $\mathrm{pCi} / \mathrm{g}$ & 0.039 & 0.076 & 0.009 & 0.010 & 0.040 & 0.013 & --- \\
\hline Gross Alpha & $\mathrm{pCi} / \mathrm{g}$ & 14.5 & 15.7 & 14.6 & 15.9 & --- & --- & --- \\
\hline Gross Beta & $\mathrm{pCi} / \mathrm{g}$ & 11.7 & 17.6 & 9.7 & 9.7 & --- & --- & --- \\
\hline Gross Gamma & $\mathrm{pCi} / \mathrm{g}$ & 8.1 & 8.8 & 4.4 & 3.6 & --- & --- & --- \\
\hline \multicolumn{9}{|c|}{$\begin{array}{l}\text { a Upper limit for background }(\mathrm{x}+2 \mathrm{~s})=\text { mean plus two tim } \\
\text { b Upper limit for background = 0.95 (two-tail) quantile (r } \\
\text { c ER Project's upper tolerance limit (Ryti et al., 1998). } \\
\text { d ER Project's upper tolerance limit (Campbell, 1998). } \\
\text { e Historical background (Purtymun et al., 1987). }\end{array}$} \\
\hline
\end{tabular}


expensive and are generally not used for all samples in the Environmental Surveillance Program. In addition, the current US EPA safe drinking limit for tritium is $20.0 \mathrm{nCi} / 1$. Hence, the current detection limit for tritium is adequate to identify and characterize unplanned releases from the Laboratory.

Uranium values in Table 11 differ because of natural variations in uranium levels contained in erodible earthen materials between sample locations (Gardner et al., 1986). For example, these authors report that natural uranium radioactivity levels in Bandelier Tuff typically vary between about 10 to $20 \mathrm{mg} / \mathrm{kg}$.

The differences for background plutonium values reported in Table 11 are most likely related to differences in analytical laboratory detection limits (or limits of quantification). The ER's limit of quantification for plutonium on soils and sediments is $100 \mathrm{fCi} / \mathrm{g}$. However, the reported detection limit for plutonium in the Environmental Surveillance Program is $5 \mathrm{fCi} / \mathrm{g}$ for river sediments and $0.1 \mathrm{fCi} / \mathrm{g}$ for reservoir sediments. Hence, the reported ER background value for plutonium-238 in Table 11 is between 6 and $23 \mathrm{fCi} / \mathrm{g}$, while it is between 54 and $68 \mathrm{fCi} / \mathrm{g}$ for plutonium-239,-240. In contrast, the reported ESH-18 background values for plutonium-238 in Table 11 are 8.7 and $1.2 \mathrm{fCi} / \mathrm{g}$ for river and reservoir sediments, respectively. For plutonium-239,-240, these values are 13.0 and $20.1 \mathrm{fCi} / \mathrm{g}$ for river and reservoir sediments, respectively. We conclude that while the ER analytical laboratory results for plutonium are perfectly acceptable for their stated purpose, their reported background values are below their limit of quantification (i.e., $100 \mathrm{fCi} / \mathrm{g}$ ). Hence, there is some added uncertainty in their results. Perhaps a better way to state the ER background value for plutonium-238 and plutonium-239,-240 in our Table 11 comparison is that they are equal to or less than 23 and $68 \mathrm{fCi} / \mathrm{g}$, respectively.

\section{CONCLUSIONS}

The computed upper limits for background values of tritium, stronium-90, cesium-137, total uranium, plutonium-238, plutonium-239,-240, americium-241, gross alpha, gross beta, and gross gamma radioactivity on river and reservoir sediments near Los Alamos, New Mexico, are summarized in Table 12. These values are based on regional sediment samples collected in five river stations and four reservoirs. Annual sediment samples were collected from river stations between 1974 and 1997. Annual sediment samples were collected from four regional reservoirs between 1979 and 1997. The upper limit for background radioactivity was assumed to equal the 0.95 quantile (two-tail) for each radionuclide. Current detection limits are also shown for comparison in Table 12. It is interesting to note that whenever the ratio of background value to detection limit falls below about 3 for a particular analyte, problems in data interpretation occasionally develop. As seen in Table 12, this ratio is less than 3 for tritium, stronium-90, plutonium-238 (river samples only), plutonium-239,-240 (river samples only), and americium-241 (reservoir samples only).

Ten stations from three different watersheds and 15 stations from five regional reservoirs have traditionally been sampled for background radioactivity on sediments in the environmental surveillance network. Historically, individual analyte values from all of these background stations have been blended together, and composite background values for sediments were established for each analyte. In this report, we separated sediments according to a river or reservoir source. In addition, we also separated individual sampling stations into upstream and downstream locations relative to the Laboratory. These new groupings resulted in five upstream and five downstream river stations, and four upstream and one downstream reservoir stations. Statistical analyses confirmed that background river and reservoir sediment samples are significantly different from one another. Furthermore, a difference between upstream and downstream station locations was also shown to be statistically significant. Hence, different background levels for individual radionuclides were computed for these different sediment sources and locations. This is an important new finding.

Suspect data have been omitted from the statistical analysis contained in this report. These data are highlighted in Appendices A and B because they have elevated uncertainties relative to other historical data or because they do not meet laboratory $\mathrm{QA} / \mathrm{QC}$ specifications. Less than $4 \%$ of all river sediment analyses and $6 \%$ of all reservoir sediment analyses were eliminated from the historical database. These highlighted data were not used in the statistical analyses to determine the upper limit of background radioactivity for river and reservoir sediments. Elimination of these data lowers the respective means and standard deviations of individual radionuclides. All data are contained in Appendices A and B, however, including those data that were eliminated in this study. Inclusion of all data was done for historical completeness. It is recommended 
Table 12. Recommended Upper Limit of Background (BGUL) Radioactivity in Sediments near Los Alamos.

\begin{tabular}{|c|c|c|c|c|}
\hline Analyte & River BGUL ${ }^{\mathbf{a}}$ & $\begin{array}{c}\text { River } \\
\text { Detection Limit }^{b}\end{array}$ & Reservoir BGUL $^{\mathrm{c}}$ & $\begin{array}{c}\text { Reservoir } \\
\text { Detection Limit }^{b}\end{array}$ \\
\hline${ }^{3} \mathrm{H}$ & $0.91 \mathrm{nCi} / 1$ & $0.70 \mathrm{nCi} / 1$ & $0.54 \mathrm{nCi} / 1$ & $0.70 \mathrm{nCi} / 1$ \\
\hline${ }^{90} \mathrm{Sr}$ & $1.02 \mathrm{pCi} / \mathrm{g}$ & $1.00 \mathrm{pCi} / \mathrm{g}$ & $1.19 \mathrm{pCi} / \mathrm{g}$ & $1.00 \mathrm{pCi} / \mathrm{g}$ \\
\hline${ }^{137} \mathrm{Cs}$ & $0.56 \mathrm{pCi} / \mathrm{g}$ & $0.05 \mathrm{pCi} / \mathrm{g}$ & $0.98 \mathrm{pCi} / \mathrm{g}$ & $0.05 \mathrm{pCi} / \mathrm{g}$ \\
\hline Total U & $4.49 \mathrm{mg} / \mathrm{kg}$ & $0.25 \mathrm{mg} / \mathrm{kg}$ & $4.58 \mathrm{mg} / \mathrm{kg}$ & $0.25 \mathrm{mg} / \mathrm{kg}$ \\
\hline${ }^{238} \mathrm{Pu}$ & $8.7 \mathrm{fCi} / \mathrm{g}$ & $5.0 \mathrm{fCi} / \mathrm{g}$ & $1.2 \mathrm{fCi} / \mathrm{g}$ & $0.1 \mathrm{fCi} / \mathrm{g}$ \\
\hline $239,240 \mathrm{Pu}$ & $13.0 \mathrm{fCi} / \mathrm{g}$ & $5.0 \mathrm{fCi} / \mathrm{g}$ & $20.1 \mathrm{fCi} / \mathrm{g}$ & $0.1 \mathrm{fCi} / \mathrm{g}$ \\
\hline${ }^{241} \mathrm{Am}$ & $0.076 \mathrm{pCi} / \mathrm{g}$ & $0.005 \mathrm{pCi} / \mathrm{g}$ & $0.010 \mathrm{pCi} / \mathrm{g}$ & $0.005 \mathrm{pCi} / \mathrm{g}$ \\
\hline Gross Alpha & $15.7 \mathrm{pCi} / \mathrm{g}$ & $1.5 \mathrm{pCi} / \mathrm{g}$ & $15.9 \mathrm{pCi} / \mathrm{g}$ & $1.5 \mathrm{pCi} / \mathrm{g}$ \\
\hline Gross Beta & $17.6 \mathrm{pCi} / \mathrm{g}$ & $1.5 \mathrm{pCi} / \mathrm{g}$ & $9.7 \mathrm{pCi} / \mathrm{g}$ & $1.5 \mathrm{pCi} / \mathrm{g}$ \\
\hline Gross Gamma & $8.8 \mathrm{pCi} / \mathrm{g}$ & $0.8 \mathrm{pCi} / \mathrm{g}$ & $3.6 \mathrm{pCi} / \mathrm{g}$ & $0.8 \mathrm{pCi} / \mathrm{g}$ \\
\hline
\end{tabular}

${ }^{a}$ Upper limit for background for river sediments $=0.95$ quantile (two-tail).

${ }^{\mathrm{b}}$ Laboratory limit of detection for sediments (see Table 1).

${ }^{\mathrm{c}}$ Upper limit for background for reservoir sediments $=0.95$ quantile (two-tail).

that data not meeting minimum laboratory QA/QC guidelines (i.e., the highlighted values in Appendices A and B) be omitted from all future studies.

The sample preparation procedure for strontium-90 analysis was modified in October 1996. The only 1996 sediment samples collected after this date came from Cochiti Reservoir. This change has resulted in higher detectable levels and uncertainties for all strontium- 90 analyses because of improvements in laboratory tracer recoveries. Previously, tracer recovery levels for strontium- 90 analyses were assumed to be $100 \%$. Hence, pre-1997 strontium-90 values may be artificially low relative to post-1997 data. In the future, all strontium-90 values need to be segregated into pre-1997 and post-1997 groups because of possible nonstationarity in the computed means and standard deviations. For this report, however, all strontium-90 sediment data collected in 1997 were simply eliminated from statistical analyses. After four to five annual sediment samples have been collected and new strontium- 90 values are available, then a new background radioactivity for strontium- 90 should be computed using only post-1997 data. This post-1997 strontium-90 mean should be compared with only post-1997 strontium-90 data. Likewise, the pre-1997 background value report here for strontium-90 in sediments should only be compared with pre-1997 data. Finally, a potential increase in mean strontium-90 values computed from post-1997 data will not indicate an upward trend in background radioactivity (or an upward trend in values from on-site sediment stations). Instead, these changes simply reflect the change in laboratory analytical procedures for this analyte.

\section{River Sediments}

Group II (River) sediments (i.e., from Rio Grande at Otowi Bridge, Rio Grande below Sandia, Rio Grande below Pajarito, Rio Grande below Ancho, and Rio Grande below Frijoles) have a statistically significant higher mean radioactivity for total uranium and plutonium-239,-240 compared with the corresponding means from Group I (River) sediments (i.e., from Rio Chama at Chamita, Rio Grande at Embudo, Rio Grande below the Cochiti Spillway, Rio Grande at Bernalillo, and the Jemez River). A higher mean radioactivity for total uranium in Group II (River) sediments simply means there is a natural uranium source in the volcanic rocks on Pajarito Plateau that are subject to erosion. The higher mean radioactivity for plutonium-239,-240 in Group II (River) sediments is probably related to natural fallout and historical Laboratory discharges. These Laboratory discharges were directed into Acid Canyon (a tributary to Pueblo Canyon) and DP Canyon (a tributary to Los Alamos Canyon). 


\section{Reservoir Sediments}

Group II (Reservoir) sediments (i.e., from Cochiti Reservoir downstream of the Laboratory) have a statistically significant higher mean radioactivity for cesium-137, plutonium-238, plutonium-239,-240, americium-241, and gross gamma radioactivity compared with the corresponding means from Group I (Reservoir) sediments (i.e., from Abiquiu, El Vado, and Heron Reservoirs on the Rio Chama and the Rio Grande Reservoir on the Rio Grande). These differences are most likely due to natural fallout and to Laboratory releases into Acid Canyon (a tributary to Pueblo Canyon) and DP Canyon (a tributary to Los Alamos Canyon).

\section{RECOMMENDATIONS}

The following recommendations are made with regard to sediment monitoring under the Laboratory's Environmental Surveillance Program.

1. The upper limits for background radioactivity values listed in Table 12 for river and reservoir sediments are recommended for general use when comparing radionuclide values obtained from sediments collected at other locations near the Laboratory. The background stations used to compute these values should be maintained. The river stations include the Rio Chama at Chamita, the Rio Grande at Embudo, the Rio Grande below the Cochiti Reservoir spillway, the Rio Grande at Bernalillo, and the Jemez River near the Jemez Pueblo. The reservoir stations include the upper, middle, and lower sediment sampling stations in Heron, El Vado, and Abiquiu Reservoirs on the Rio Chama and the Rio Grande Reservoir on the Rio Grande.

2. Annual sediment sampling from the Group II (River) and Group II (Reservoir) stations should also be continued. However, these analyses should not be included in future background calculations.

3. Consideration should be given to increasing the river sediment sample size to $1 \mathrm{~kg}$ if this is practical. A larger sample would lower the river sediment detection limit from $5.0 \mathrm{fCi} / \mathrm{g}$ to $0.1 \mathrm{fCi} / \mathrm{g}$ for plutonium238 and plutonium-239,-240.

4. The sediment station located on the Rio Grande below the Cochiti Reservoir Spillway should be moved approximately $1-2 \mathrm{~km}$ farther downstream so that finer-grained sediments can be routinely collected at this station. The fast-moving waters immediately below the spillway are not conducive to sampling finegrained sediments. Hence, historical records may not be representative of radiochemicals and sediments leaving Cochiti Reservoir.

5. In general, tritium analyses for sediments in this study have a relatively high laboratory detection limit compared with natural tritium levels typically found in the environment near Los Alamos. However, these analyses are still very useful because they are intended to monitor and document unplanned releases from the Laboratory.

\section{ACKNOWLEDGMENTS}

This work was supported by the Environmental Surveillance Program within the Water Quality and Hydrology Group (ESH-18) at Los Alamos National Laboratory. All of the sediment sampling stations listed in this report were originally located by William D. Purtymun, who started the soil and sediment monitoring program in the early 1970s. We also appreciate the helpful review comments and discussion provided by Ken Mullen, Bruce Gallaher, Larry Pratt, William Turney, and John Nyhan. 


\section{REFERENCES}

Aarkrog, A., "Source Terms and Inventories of Anthropogenic Radionuclides," Nordic Society for Radiation Protection, Risø National Laboratory, Roskilde, Denmark, report DK-4000 (1991).

Adams, A. I., F. Goff, and D. Counce, "Chemical and Isotopic Variations of Precipitation in the Los Alamos Region, New Mexico,” Los Alamos National Laboratory report LA-12895-MS (February 1995), 35 pp.

Blake, W. D., F. Goff, A. I. Adams, and D. Counce, "Environmental Geochemistry for Surface and Subsurface Waters in the Pajarito Plateau and Outlying Area, New Mexico," Los Alamos National Laboratory report LA-12912-MS (May 1995).

Campbell, K., "Baseline Data for Fallout Radionuclides at LANL," Los Alamos National Laboratory document LA-UR-98-0958 (April 23, 1998), 30 pp.

Conover, W. J., Practical Nonparametric Statistics (John Wiley and Sons, Inc., New York, 1998), 584 pp.

Environmental Assessments and Resource Evaluation Group, "Environmental Surveillance at Los Alamos during 1994,” Los Alamos National Laboratory report LA-13047-ENV (July 1996).

Environmental Protection Agency, "40 CFR Parts 141 and 142, National Primary Drinking Water Regulations, Radionuclides, Proposed Rule," Federal Register, Vol. 50, No. 138, pp. 33050-33127 (July 18, 1991).

Environmental Protection Group, “Environmental Surveillance at Los Alamos During 1989," Los Alamos National Laboratory report LA-12000-ENV (December 1990).

Environmental Protection Group, "Environmental Surveillance at Los Alamos during 1990," Los Alamos National Laboratory report LA-12271-MS (March 1992).

Environmental Protection Group, "Environmental Surveillance at Los Alamos during 1991," Los Alamos National Laboratory report LA-12572-ENV (August 1993).

Environmental Protection Group, "Environmental Surveillance at Los Alamos during 1992," Los Alamos National Laboratory report LA-12764-ENV (July 1994).

Environmental Protection Group, "Environmental Surveillance at Los Alamos during 1993," Los Alamos National Laboratory report LA-12973-ENV (October 1995).

Environmental Restoration Project, "Derivation and Use of Radionuclide Screening Action Levels," Los Alamos National Laboratory document LA-UR-01-990 (March 2001).

Environmental Studies Group, "Environmental Surveillance at Los Alamos During 1974," Los Alamos Scientific Laboratory report LA-5977-PR (May 1975).

Environmental Studies Group, "Environmental Surveillance at Los Alamos During 1975," Los Alamos Scientific Laboratory report LA-6321-MS (April 1976).

Environmental Studies Group, "Environmental Surveillance at Los Alamos During 1976," Los Alamos Scientific Laboratory report LA-6801-MS (April 1977).

Environmental Surveillance and Compliance Programs, "Environmental Surveillance and Compliance at Los Alamos during 1996," Los Alamos National Laboratory report LA-13343-ENV (September 1997). 
Environmental Surveillance Group, "Environmental Surveillance at Los Alamos During 1977," Los Alamos Scientific Laboratory report LA-7263-MS (April 1978).

Environmental Surveillance Group, "Environmental Surveillance at Los Alamos During 1978," Los Alamos Scientific Laboratory report LA-7800-ENV (April 1979).

Environmental Surveillance Group, “Environmental Surveillance at Los Alamos During 1979,” Los Alamos Scientific Laboratory report LA-8200-ENV (April 1980).

Environmental Surveillance Group, "Environmental Surveillance at Los Alamos During 1980," Los Alamos National Laboratory report LA-8810-ENV (April 1981).

Environmental Surveillance Group, "Environmental Surveillance at Los Alamos During 1981," Los Alamos National Laboratory report LA-9349-ENV (April 1982).

Environmental Surveillance Group, "Environmental Surveillance at Los Alamos During 1982," Los Alamos National Laboratory report LA-9762-ENV (April 1983).

Environmental Surveillance Group, "Environmental Surveillance at Los Alamos During 1983," Los Alamos National Laboratory report LA-10100-ENV (April 1984).

Environmental Surveillance Group, "Environmental Surveillance at Los Alamos During 1984," Los Alamos National Laboratory report LA-10421-ENV (April 1985).

Environmental Surveillance Group, "Environmental Surveillance at Los Alamos During 1985," Los Alamos National Laboratory report LA-10721-ENV (April 1986).

Environmental Surveillance Group, "Environmental Surveillance at Los Alamos During 1986," Los Alamos National Laboratory report LA-10992-ENV (April 1987).

Environmental Surveillance Group, "Environmental Surveillance at Los Alamos During 1987," Los Alamos National Laboratory report LA-11306-ENV (May 1988).

Environmental Surveillance Group, "Environmental Surveillance at Los Alamos During 1988," Los Alamos National Laboratory report LA-11628-ENV (June 1989).

Environmental Surveillance Program, "Environmental Surveillance at Los Alamos during 1995," Los Alamos National Laboratory report LA-13210-ENV (October 1996).

Environmental Surveillance Program, "Environmental Surveillance at Los Alamos during 1997," Los Alamos National Laboratory report LA-13487-ENV (September 1998).

Fresquez, P. R., D. R. Armstrong, and J. G. Salazar, "Radionuclide Concentration in Game and Nongame Fish Upstream and Downstream of Los Alamos National Laboratory: 1981 to 1993," Los Alamos National Laboratory report LA-12818-MS (August 1994).

Fresquez, P. R., M. A. Mullen, J. K. Ferenbaugh, and R. A. Perona, "Radionuclides and Radioactivity in Soils Within and Around Los Alamos National Laboratory, 1974 Through 1994: Concentrations, Trends, and Dose Comparisons," Los Alamos National Laboratory report LA-13149-MS (April 1996), 42 pp.

Gardner, J. N., F. Goff, S. Garcia, and R. C. Hagan, "Stratigraphic Relations and Lithologic Variations in the Jemez Volcanic Field, New Mexico," Journal of Geophysical Research 91 (B2), 1763-1778 (1986). 
Gallaher, B. M., D. W. Efurd, D. J. Rokop, T. M. Benjamin, and A. K. Stoker, "Survey of Plutonium and Uranium Atom Ratios and Activity Levels in Mortandad Canyon," Los Alamos National Laboratory report LA-13379-MS (October 1997).

Gallaher, B. M., D. W. Efurd, D. J. Rokop, and T. M. Benjamin, "Plutonium and Uranium Atom Ratios and Activity Levels in Cochiti Lake Bottom Sediments Provided by Pueblo de Cochiti," Los Alamos National Laboratory report LA-13605-MS (May 1999).

Gautier, M. A., and E. S. Gladney, Eds., "Health and Environmental Chemistry: Analytical Techniques, Data Management, and Quality Assurance," Los Alamos National Laboratory report LA-10300-M, Volumes 1-4 and their revisions (1993).

Gilbert, R. O., "Recommendations Concerning the Computation and Reporting of Counting Statistics for the Nevada AppliedEcology Group,"Battelle Pacific NorthwestLaboratories reportBNWL-B-368(September 1975).

Gilbert, R. O., Statistical Methods for Environmental Pollution Monitoring (Van Nostrand Reinhold, New York, 1987), 320 pp.

Glymph, L. M., "Summary: Sedimentation in Reservoirs," in Man-Made Lakes, Their Problems and Environmental Effects, edited by W. C. Ackermann, G. F. White, E. B. Worthington, and J. L. Ivens, American Geophysical Union, Geophysical Monograph 17, Washington, D.C. (1973), pp. 342-348.

Graf, W. L., "Geomorphology of Plutonium in the Northern Rio Grande," Los Alamos National Laboratory document LA-UR-93-1963 (1993).

Graf, W. L., "Transport and Deposition of Plutonium-Contaminated Sediments by Fluvial Processes, Los Alamos Canyon, New Mexico," Geological Society of America Bulletin 108 (10), 1342-1355 (1996).

Holleman, J. W., P. A. Quiggins, B. D. Chilton, M. S. Uziel, H. A. Pfuderer, and J. A. Longmire, "Worldwide Fallout of Plutonium from Nuclear Weapons Tests," Oak Ridge National Laboratory report ORNL-6315 (1987), $288 \mathrm{pp}$.

Kirhner, G., and C. Noack, "Core History and Nuclide Inventory of Chernobyl Core at the Time of Accident," Nuclear Safety 29 (1), 1-5 (1988).

Krey, P. W., “Atmospheric Burn-up of a Plutonium-238 Generator,” Science 158, 769-771 (1967).

Lilliefors, H. W., "On the Kolmogorov-Smirnov Test for Normality with Mean and Variance Unknown," Journal of the American Statistical Association 62, 399-402 (1967).

Lilliefors, H. W., Correction to the paper, "On the Kolmogorov-Smirnov Test for Normality with Mean and Variance Unknown,” Journal of the American Statistical Association 64, 1702 (1969).

Longmire, P. A., S. L. Reneau, P. M. Watt, L. D. McFadden, J. N. Gardner, C. J. Duffy, and R. T. Ryti, "Natural Background Geochemistry, Geomorphology, and Pedogenesis of Selected Soil Profiles and Bandelier Tuff, Los Alamos, New Mexico,” Los Alamos National Laboratory report LA-12913-MS (1996), $176 \mathrm{pp}$.

Norris, R. S., A. S. Burrows, and R. W. Fieldhouse, Nuclear Weapons Databook, Vol. 5, British, French, and Chinese Nuclear Weapons (Westview Press, Boulder, Colorado, 1994).

Perkins, R. W., and C. W. Thomas, "Worldwide Fallout," in "Transuranic Elements in the Environment," W. C. Hanson, Ed., US Dept. of Energy report DOE/TIC-22800 (1980), pp. 53-82. 
Pinder, J. E., and M. H. Smith, "Frequency Distributions of Radiocesium Concentrations in Soil and Biota," in "Mineral Cycling in Southeastern Ecosystems," F. G. Howell, J. B. Gentry, and M. H. Smith, Eds., US Energy Research and Development Administration report CONF-740513 (1975), pp. 107-125.

Purtymun, W. D., R. J. Peters, and A. K. Stoker, "Radioactivity in Soils and Sediments in and Adjacent to the Los Alamos Area, 1974-77," Los Alamos Scientific Laboratory report LA-8234-MS (February 1980).

Purtymun, W. D., R. J. Peters, T. E. Buhl, M. N. Maes, and F. H. Brown, "Background Concentrations of Radionuclides in Soil and River Sediments in Northern New Mexico, 1974-1986," Los Alamos National Laboratory report LA-11134-MS (November 1987).

Purtymun, W. D., R. J. Peters, and M. N. Maes, "Plutonium Deposition and Distribution from Worldwide Fallout in Northern New Mexico and Southern Colorado," Los Alamos National Laboratory report LA-11794-MS (July 1990).

Rogers, D. B., "Impact of Tritium Disposal on Surface Water and Groundwater at Los Alamos National Laboratory thorough 1997," Los Alamos National Laboratory report LA-13465-SR (July 1998), 31 pp.

Ryti, R. T., P. A. Longmire, D. E. Broxton, S. L. Reneau, and E. V. McDonald, "Inorganic and Radionuclide Background Data for Soils, Canyon Sediments, and Bandelier Tuff at Los Alamos National Laboratory," Los Alamos National Laboratory document LA-UR-98-4847 (1998).

Stoker, A. K., A. J. Ahlquist, W. R. Hansen, and A. D. Talley, "Formerly Utilized MED/AEC Sites Remedial Action Program," Los Alamos National Laboratory report LA-8890-ENV (May 1981), 252 pp.

Tracy, B. L., F. A. Prantl, and J. M. Quinn, "Health Impacts of Radioactive Debris from the Satellite Kosmos 954," Health Physics 47, 225-233 (1984).

US Environmental Protection Agency, "Federal Radiation Protection Guidance for Exposure of the General Public," Notice in Federal Register, December 23, 1994.

Wilson, J.T., and P.C. van Metre, "Deposition and Chemistry of Bottom Sediments in Cochiti Lake, NorthCentral New Mexico,” US Geological Survey, Water Resources Investigations report 99-4258 (2000), 31 pp. 
APPENDIX A

RADIOCHEMICAL ANALYSES FOR RIVER SEDIMENTS 
Appendix A. Radiochemical Analyses of River Sediments

\begin{tabular}{|c|c|c|c|c|c|c|c|c|c|c|c|c|c|c|c|c|c|c|c|c|}
\hline \multicolumn{21}{|c|}{ Table A-1. Sediment Station: Rio Chama at Chamita } \\
\hline \multirow{3}{*}{$\begin{array}{c}\text { Sample } \\
\text { Date }\end{array}$} & \multirow{3}{*}{$\begin{array}{c}{ }^{3} \mathrm{H} \\
(\mathrm{nCi} / \mathrm{I}) \\
\end{array}$} & \multirow{3}{*}{$\pm \mathbf{u}$} & \multirow{3}{*}{$\begin{array}{c}{ }^{90} \mathrm{Sr} \\
(\mathrm{pCi} / \mathrm{g})\end{array}$} & \multirow{3}{*}{$\pm \mathbf{u}$} & \multirow{3}{*}{$\begin{array}{c}{ }^{137} \mathrm{Cs} \\
(\mathrm{pCi} / \mathrm{g})\end{array}$} & \multirow{3}{*}{$\pm \mathbf{u}$} & \multirow{3}{*}{$\begin{array}{l}\text { Total U } \\
(\mathbf{m g} / \mathbf{k g})\end{array}$} & \multirow{3}{*}{$\pm \mathbf{u}$} & \multirow{3}{*}{$\begin{array}{c}{ }^{238} \mathrm{Pu} \\
(\mathrm{pCi} / \mathbf{g})\end{array}$} & \multirow{3}{*}{$\pm \mathbf{u}$} & \multirow{3}{*}{$\begin{array}{l}{ }^{23,2,240} \mathrm{Pu} \\
(\mathrm{pCi} / \mathrm{g})\end{array}$} & \multirow{3}{*}{$\pm \mathbf{u}$} & \multirow{3}{*}{$\begin{array}{l}{ }^{241} \mathrm{Am} \\
(\mathrm{pCi} / \mathrm{g})\end{array}$} & \multirow{3}{*}{$\pm \mathbf{u}$} & Gross & & Gross & & Gross & \\
\hline & & & & & & & & & & & & & & & Alpha & $\pm \mathbf{u}$ & Beta & $\pm \mathbf{u}$ & Gamma & $\pm \mathbf{u}$ \\
\hline & & & & & & & & & & & & & & & $(\mathrm{pCi} / \mathrm{g})$ & & $(\mathrm{pCi} / \mathrm{g})$ & & $(\mathrm{pCi} / \mathrm{g})$ & \\
\hline 07/01/74 & & & & & 0.08 & 0.12 & & & 0.000 & 0.001 & 0.001 & 0.001 & & & & & & & & \\
\hline 05/01/75 & & & & & & & & & 0.002 & 0.002 & 0.001 & 0.001 & & & & & & & & \\
\hline $09 / 01 / 75$ & & & & & & & & & -0.001 & 0.001 & 0.001 & 0.001 & & & & & & & & \\
\hline 03/29/76 & 1.3 & 0.3 & 1.14 & 1.06 & 0.19 & 0.02 & 1.00 & 0.20 & 0.000 & 0.001 & 0.002 & 0.001 & & & 9.0 & 2.0 & 5.2 & 0.6 & & \\
\hline $10 / 04 / 76$ & & & 0.01 & 0.07 & & & & & 0.000 & 0.001 & 0.000 & 0.001 & & & 0.5 & 0.4 & 1.1 & 0.3 & & \\
\hline 03/07/77 & & & -0.02 & 0.06 & 0.08 & 0.03 & 0.90 & 0.70 & 0.001 & 0.002 & 0.001 & 0.002 & & & 1.6 & 0.5 & 1.6 & 0.3 & & \\
\hline $10 / 18 / 77$ & 3.5 & 0.4 & 0.00 & 0.10 & 0.06 & 0.03 & 1.50 & 0.50 & 0.000 & 0.001 & 0.001 & 0.001 & & & 0.9 & 0.4 & 1.7 & 0.4 & 0.4 & 0.1 \\
\hline 03/06/78 & & & & & 0.00 & 0.03 & & & 0.000 & 0.001 & -0.002 & 0.002 & & & 2.4 & 0.6 & 2.8 & 0.5 & 1.3 & 0.1 \\
\hline $03 / 08 / 79$ & & & -0.15 & 0.09 & 0.05 & 0.06 & 1.10 & 0.10 & 0.000 & 0.000 & 0.000 & 0.001 & & & 1.0 & 0.4 & 1.5 & 0.4 & -0.1 & 0.1 \\
\hline $02 / 26 / 80$ & & & 0.82 & 0.11 & 0.18 & 0.03 & 1.50 & 0.20 & 0.000 & 0.001 & 0.003 & 0.002 & 0.080 & 0.030 & 3.0 & 0.7 & 2.9 & 0.5 & 1.8 & 0.1 \\
\hline $03 / 26 / 81$ & & & -0.10 & 0.09 & 0.15 & 0.12 & & & 0.000 & 0.000 & 0.003 & 0.001 & & & 3.5 & 1.0 & 5.7 & 0.7 & 1.3 & 0.1 \\
\hline 03/01/82 & & & & & & & & & & & & & & & & & & & & \\
\hline $02 / 01 / 83$ & & & & & 0.10 & 0.03 & 1.90 & 0.10 & 0.004 & 0.001 & 0.011 & 0.002 & & & & & & & 2.2 & 0.1 \\
\hline $02 / 22 / 84$ & & & & & 0.27 & 0.15 & 1.30 & 0.10 & 0.000 & 0.001 & 0.000 & 0.002 & & & & & & & 2.0 & 0.1 \\
\hline $04 / 29 / 85$ & & & & & 0.22 & 0.07 & 1.90 & 0.20 & 0.001 & 0.001 & 0.003 & 0.001 & & & & & & & 1.6 & 0.2 \\
\hline $02 / 01 / 86$ & & & & & 0.23 & 0.09 & 2.30 & 0.20 & -0.001 & 0.001 & 0.001 & 0.001 & & & & & & & 1.9 & 0.2 \\
\hline 03/01/87 & & & & & 0.00 & 0.06 & 1.90 & 0.20 & -0.001 & 0.001 & -0.002 & 0.001 & -0.160 & 0.081 & & & & & -3.5 & 0.4 \\
\hline 03/28/88 & & & & & 0.16 & 0.08 & 1.30 & 0.20 & 0.000 & 0.001 & 0.002 & 0.001 & & & & & & & -1.1 & 0.3 \\
\hline $03 / 27 / 89$ & & & & & 0.20 & 0.12 & 1.80 & 0.20 & 0.002 & 0.001 & 0.004 & 0.001 & & & & & & & 1.2 & 0.3 \\
\hline $04 / 02 / 90$ & & & & & 0.13 & 0.09 & & & 0.000 & 0.000 & 0.001 & 0.001 & & & & & & & 4.0 & 0.3 \\
\hline 03/01/91 & & & 0.10 & 0.20 & 0.10 & 0.10 & 2.60 & 0.30 & 0.001 & 0.001 & 0.002 & 0.001 & & & 3.0 & 1.0 & 3.0 & 0.0 & 1.0 & 0.0 \\
\hline 03/01/92 & -0.3 & 0.3 & 0.20 & 0.20 & 0.00 & 0.10 & 2.90 & 0.30 & 0.009 & 0.008 & 0.003 & 0.006 & 0.139 & 0.077 & 6.0 & 1.0 & 3.0 & 1.0 & 9.0 & 1.0 \\
\hline $10 / 22 / 93$ & -0.1 & 0.3 & 0.00 & 0.20 & 0.10 & 0.00 & 1.90 & 0.10 & 0.003 & 0.030 & 0.006 & 0.020 & 0.002 & 0.030 & 2.0 & 1.0 & 2.0 & 0.0 & -1.0 & 0.0 \\
\hline 07/24/94 & 0.2 & 0.3 & -1.70 & 1.90 & 0.08 & 0.03 & 1.00 & 0.30 & 0.004 & 0.030 & 0.003 & 0.020 & 0.004 & 0.030 & 3.0 & 1.0 & 3.0 & 0.0 & 2.0 & 0.0 \\
\hline $03 / 23 / 95$ & 0.0 & 0.4 & 10.80 & 0.70 & 0.05 & 0.02 & 0.61 & 0.10 & 0.004 & 0.001 & 0.002 & 0.001 & 0.003 & 0.001 & 0.8 & 0.2 & 0.5 & 0.1 & 1.4 & 0.2 \\
\hline 05/09/96 & 0.0 & 1.0 & 0.70 & 0.30 & 0.06 & 0.02 & 1.35 & 0.14 & 0.001 & 0.001 & 0.002 & 0.001 & 0.002 & 0.001 & 1.5 & 0.4 & 1.4 & 0.2 & 2.8 & 0.3 \\
\hline 05/12/97 & 0.0 & 0.8 & -0.09 & 1.03 & 0.09 & 0.01 & 1.71 & 0.17 & 0.002 & 0.001 & 0.003 & 0.001 & 0.006 & 0.001 & 1.0 & 9.5 & 1.3 & 0.8 & 2.2 & 0.2 \\
\hline Table A-2. Sta & tistical Sum & nary for & r Rio Chan & a at $\mathrm{Ch}$ & amita & & & & & & & & & & & & & & & \\
\hline & & & & & & & & & & & & & & & Gross & & Gross & & Gross & \\
\hline & ${ }^{3} \mathbf{H}$ & $\pm \mathbf{u}$ & ${ }^{90} \mathrm{Sr}$ & $\pm \mathbf{u}$ & ${ }^{137} \mathrm{Cs}$ & $\pm \mathbf{u}$ & Total U & $\pm \mathbf{u}$ & ${ }^{238} \mathbf{P u}$ & $\pm \mathbf{u}$ & ${ }^{239,240} \mathrm{Pu}$ & $\pm \mathbf{u}$ & ${ }^{241} \mathrm{Am}$ & $\pm \mathbf{u}$ & Alpha & $\pm \mathbf{u}$ & Beta & $\pm \mathbf{u}$ & Gamma & $\pm \mathbf{u}$ \\
\hline Statistic & $(\mathrm{nCi} / \mathrm{l})$ & & $(\mathrm{pCi} / \mathrm{g})$ & & $(\mathrm{pCi} / \mathrm{g})$ & & $(\mathrm{mg} / \mathrm{kg})$ & & $(\mathrm{pCi} / \mathrm{g})$ & & $(\mathrm{pCi} / \mathrm{g})$ & & $(\mathrm{pCi} / \mathrm{g})$ & & $(\mathrm{pCi} / \mathrm{g})$ & & $(\mathrm{pCi} / \mathrm{g})$ & & $(\mathrm{pCi} / \mathrm{g})$ & \\
\hline Mean (x) & 0.0 & 0.5 & 0.16 & 0.14 & 0.11 & 0.06 & 1.60 & 0.23 & 0.001 & 0.003 & 0.002 & 0.003 & 0.016 & 0.016 & 2.6 & 1.3 & 2.4 & 0.4 & 1.5 & 0.2 \\
\hline Median (m) & 0.0 & 0.4 & 0.01 & 0.11 & 0.10 & 0.06 & 1.50 & 0.20 & 0.000 & 0.001 & 0.002 & 0.001 & 0.004 & 0.016 & 2.0 & 0.7 & 2.0 & 0.4 & 1.5 & 0.1 \\
\hline Std Dev (s) & 0.2 & 0.3 & 0.33 & 0.08 & 0.08 & 0.04 & 0.59 & 0.15 & 0.002 & 0.008 & 0.003 & 0.005 & 0.031 & 0.016 & 2.3 & 2.3 & 1.5 & 0.3 & 2.4 & 0.2 \\
\hline Count & 6 & 6 & 10 & 10 & 23 & 23 & 19 & 19 & 26 & 26 & 26 & 26 & 6 & 6 & 15 & 15 & 15 & 15 & 20 & 20 \\
\hline Min & -0.3 & 0.3 & -0.15 & 0.06 & 0.00 & 0.00 & 0.61 & 0.10 & -0.001 & 0.000 & -0.002 & 0.001 & 0.002 & 0.001 & 0.5 & 0.2 & 0.5 & 0.0 & -3.5 & 0.0 \\
\hline Max & 0.2 & 1.0 & 0.82 & 0.30 & 0.27 & 0.15 & 2.90 & 0.70 & 0.009 & 0.030 & 0.011 & 0.020 & 0.080 & 0.030 & 9.0 & 9.5 & 5.7 & 1.0 & 9.0 & 1.0 \\
\hline$(x+2 s)$ & 0.3 & & 0.82 & & 0.26 & & 2.79 & & 0.006 & & 0.007 & & 0.079 & & 7.2 & & 5.4 & & 6.3 & \\
\hline$(x+5 s)$ & 0.8 & & 1.82 & & 0.49 & & 4.56 & & 0.012 & & 0.015 & & 0.173 & & 14.0 & & 9.7 & & 13.4 & \\
\hline BGUL (95\%) & 3.5 & & 0.82 & & 0.27 & & 2.90 & & 0.008 & & 0.010 & & 0.080 & & 9.0 & & 5.7 & & 9.0 & \\
\hline Notes: & ) Individu & high & llighted ana & v & is have ele & d un & tainities & & meet labora & QA/C & QC control & ific & ss. Data al & ot incl & Ided in stat & tical sul & nmary. & & & \\
\hline & & & & & & & & & & & & & & & & & & & & \\
\hline
\end{tabular}


Appendix A. Radiochemical Analyses of River Sediments

\begin{tabular}{|c|c|c|c|c|c|c|c|c|c|c|c|c|c|c|c|c|c|c|c|c|}
\hline \multicolumn{21}{|c|}{ Table A-3. Sediment Station: Rio Grande at Embudo } \\
\hline & & & & & & & & & & & & & & & Gross & & Gross & & Gross & \\
\hline Sample & ${ }^{3} \mathbf{H}$ & $\pm \mathbf{u}$ & ${ }^{90} \mathrm{Sr}$ & $\pm \mathbf{u}$ & ${ }^{137} \mathrm{Cs}$ & $\pm \mathbf{u}$ & Total U & $\pm \mathbf{u}$ & ${ }^{238} \mathbf{P u}$ & $\pm \mathbf{u}$ & ${ }^{239,240} \mathbf{P u}$ & $\pm \mathbf{u}$ & ${ }^{241} \mathrm{Am}$ & $\pm \mathbf{u}$ & Alpha & $\pm \mathbf{u}$ & Beta & $\pm \mathbf{u}$ & Gamma & $\pm \mathbf{u}$ \\
\hline Date & $(\mathrm{nCi} / \mathrm{l})$ & & $(\mathrm{pCi} / \mathrm{g})$ & & $(\mathrm{pCi} / \mathrm{g})$ & & $(\mathrm{mg} / \mathrm{kg})$ & & $(\mathrm{pCi} / \mathrm{g})$ & & $(\mathrm{pCi} / \mathrm{g})$ & & $(\mathrm{pCi} / \mathrm{g})$ & & $(\mathrm{pCi} / \mathrm{g})$ & & $(\mathrm{pCi} / \mathrm{g})$ & & $(\mathrm{pCi} / \mathrm{g})$ & \\
\hline 07/01/74 & & & & & 0.56 & 0.13 & & & 0.001 & 0.002 & 0.005 & 0.002 & & & & & & & & \\
\hline 05/01/75 & & & & & & & & & -0.001 & 0.001 & 0.002 & 0.002 & & & & & & & & \\
\hline $09 / 01 / 75$ & & & & & & & & & 0.000 & 0.001 & 0.011 & 0.002 & & & & & & & & \\
\hline 03/29/76 & 0.2 & 0.3 & 1.05 & 1.70 & 0.14 & 0.02 & 0.30 & 0.30 & 0.000 & 0.001 & 0.002 & 0.001 & & & 4.2 & 1.1 & 3.5 & 0.5 & & \\
\hline $10 / 04 / 76$ & & & 0.02 & 0.03 & & & & & 0.001 & 0.002 & 0.002 & 0.001 & & & 0.7 & 0.5 & 1.6 & 0.4 & & \\
\hline 03/07/77 & & & -0.04 & 0.06 & 0.14 & 0.02 & 2.70 & 1.20 & 0.001 & 0.002 & 0.002 & 0.004 & & & 1.7 & 0.5 & 1.2 & 0.3 & & \\
\hline 10/18/77 & 2.6 & 0.4 & 0.77 & 0.11 & 0.09 & 0.03 & 1.70 & 0.60 & -0.001 & 0.001 & 0.001 & 0.001 & & & 1.5 & 0.5 & 1.4 & 0.4 & 0.5 & 0.1 \\
\hline 03/06/78 & & & & & 0.26 & 0.08 & & & -0.002 & 0.001 & -0.006 & 0.002 & & & 1.9 & 0.5 & 1.7 & 0.4 & 2.9 & 0.1 \\
\hline $03 / 07 / 79$ & & & -7.00 & 0.07 & 0.24 & 0.10 & 3.00 & 0.30 & 0.000 & 0.000 & 0.002 & 0.000 & & & 4.7 & 1.2 & 3.6 & 0.6 & 2.9 & 0.1 \\
\hline $02 / 26 / 80$ & & & 0.10 & 0.04 & 0.34 & 0.09 & 4.60 & 0.50 & -0.002 & 0.002 & 0.004 & 0.002 & & & 4.7 & 1.1 & 5.5 & 0.7 & 5.8 & 0.1 \\
\hline 03/16/81 & & & 0.06 & 0.06 & 0.14 & 0.05 & & & 0.000 & 0.000 & 0.003 & 0.001 & & & 8.0 & 1.9 & 7.2 & 0.9 & 3.4 & 0.1 \\
\hline 03/01/82 & & & & & 0.23 & 0.04 & 1.90 & 0.20 & 0.000 & 0.000 & 0.002 & 0.001 & & & 3.8 & 1.8 & 4.6 & 1.2 & & \\
\hline $02 / 01 / 83$ & & & & & 0.12 & 0.22 & 2.30 & 0.10 & 0.004 & 0.001 & 0.003 & 0.001 & & & & & & & 2.4 & 0.1 \\
\hline $02 / 22 / 84$ & 2.7 & 0.8 & & & 0.27 & 0.15 & 2.70 & 0.20 & 0.001 & 0.004 & 0.002 & 0.004 & & & & & & & 4.6 & 0.6 \\
\hline $04 / 29 / 85$ & & & & & 0.09 & 0.07 & 2.90 & 0.70 & 0.000 & 0.001 & 0.004 & 0.003 & & & & & & & 2.8 & 0.4 \\
\hline $02 / 01 / 86$ & & & & & 0.05 & 0.06 & 2.80 & 0.30 & 0.000 & 0.002 & 0.003 & 0.001 & & & & & & & 3.1 & 0.4 \\
\hline 03/01/87 & & & & & 0.00 & 0.06 & 1.90 & 0.20 & 0.000 & 0.001 & 0.000 & 0.001 & -0.146 & 0.177 & & & & & -4.1 & 0.7 \\
\hline 03/28/88 & & & & & 0.10 & 0.09 & 1.00 & 0.20 & 0.006 & 0.002 & 0.002 & 0.001 & & & & & & & -0.8 & 0.5 \\
\hline $03 / 27 / 89$ & & & & & 0.16 & 0.63 & 2.00 & 0.20 & 0.005 & 0.001 & 0.002 & 0.001 & & & & & & & 0.5 & 0.4 \\
\hline $04 / 02 / 90$ & & & & & 0.12 & 0.08 & & & 0.001 & 0.001 & 0.002 & 0.001 & & & & & & & 4.5 & 0.6 \\
\hline 03/01/91 & & & 0.10 & 0.20 & 0.10 & 0.10 & 2.60 & 0.30 & 0.011 & 0.002 & 0.004 & 0.001 & & & 2.0 & 1.0 & 3.0 & 0.0 & 0.0 & 0.0 \\
\hline 03/01/92 & -0.3 & 0.3 & 0.10 & 0.20 & 0.10 & 0.10 & 2.90 & 0.30 & 0.005 & 0.003 & 0.004 & 0.002 & 0.086 & 0.074 & 6.0 & 1.0 & 2.0 & 1.0 & 8.0 & 1.0 \\
\hline $10 / 23 / 93$ & 0.1 & 0.3 & 0.00 & 0.20 & 0.10 & 0.00 & 1.20 & 0.10 & 0.005 & 0.030 & 0.006 & 0.020 & 0.001 & 0.030 & 3.0 & 1.0 & 3.0 & 0.0 & 0.0 & 0.0 \\
\hline $07 / 25 / 94$ & 0.3 & 0.3 & 0.20 & 0.20 & 0.15 & 0.05 & 1.20 & 0.40 & 0.001 & 0.030 & 0.004 & 0.020 & & & & & & & & \\
\hline 03/23/95 & 0.0 & 0.3 & 0.10 & 0.20 & 0.05 & 0.02 & 1.39 & 0.26 & 0.001 & 0.001 & 0.002 & 0.001 & 0.003 & 0.003 & 1.9 & 0.5 & 1.6 & 0.2 & 1.8 & 0.3 \\
\hline 05/09/96 & 1.0 & 1.0 & 0.30 & 0.20 & 0.07 & 0.02 & 2.03 & 0.20 & 0.001 & 0.001 & 0.003 & 0.001 & 0.003 & 0.005 & 3.5 & 1.6 & 2.4 & 0.3 & 1.7 & 0.2 \\
\hline 05/12/97 & -0.3 & 0.7 & 0.67 & 0.59 & 0.11 & 0.02 & 1.86 & 0.19 & 0.001 & 0.001 & 0.003 & 0.001 & 0.002 & 0.001 & 2.8 & 2.1 & 2.1 & 1.0 & 2.4 & 0.2 \\
\hline \multicolumn{21}{|c|}{ Table A-4. Statistical Summary for Rio Grande at Embudo } \\
\hline & & & & & & & & & & & & & & & Gross & & Gross & & Gross & \\
\hline & ${ }^{3} \mathrm{H}$ & $\pm \mathbf{u}$ & ${ }^{90} \mathrm{Sr}$ & $\pm \mathbf{u}$ & ${ }^{137} \mathrm{Cs}$ & $\pm \mathbf{u}$ & Total U & $\pm \mathbf{u}$ & ${ }^{238} \mathbf{P u}$ & $\pm \mathbf{u}$ & ${ }^{239,240} \mathbf{P u}$ & $\pm \mathbf{u}$ & ${ }^{241} \mathrm{Am}$ & $\pm \mathbf{u}$ & Alpha & $\pm \mathbf{u}$ & Beta & $\pm \mathbf{u}$ & Gamma & $\pm \mathbf{u}$ \\
\hline Statistic & $(\mathrm{nCi} / \mathrm{l})$ & & $(\mathrm{pCi} / \mathrm{g})$ & & $(\mathrm{pCi} / \mathrm{g})$ & & $(\mathrm{mg} / \mathrm{kg})$ & & $(\mathrm{pCi} / \mathrm{g})$ & & $(\mathrm{pCi} / \mathrm{g})$ & & $(\mathrm{pCi} / \mathrm{g})$ & & $(\mathrm{pCi} / \mathrm{g})$ & & $(\mathrm{pCi} / \mathrm{g})$ & & $(\mathrm{pCi} / \mathrm{g})$ & \\
\hline Mean (x) & 0.1 & 0.5 & 0.16 & 0.14 & 0.16 & 0.09 & 2.15 & 0.34 & 0.001 & 0.003 & 0.003 & 0.003 & 0.002 & 0.010 & 3.4 & 1.1 & 3.0 & 0.5 & 2.2 & 0.3 \\
\hline Median (m) & 0.1 & 0.3 & 0.10 & 0.20 & 0.12 & 0.07 & 2.02 & 0.28 & 0.001 & 0.001 & 0.002 & 0.001 & 0.003 & 0.004 & 3.0 & 1.0 & 2.4 & 0.4 & 2.4 & 0.2 \\
\hline Std Dev (s) & 0.5 & 0.3 & 0.22 & 0.08 & 0.12 & 0.12 & 0.94 & 0.26 & 0.003 & 0.008 & 0.003 & 0.005 & 0.001 & 0.014 & 1.9 & 0.5 & 1.7 & 0.4 & 2.6 & 0.3 \\
\hline Count & 6 & 6 & 11 & 11 & 24 & 24 & 20 & 20 & 27 & 27 & 27 & 27 & 4 & 4 & 15 & 15 & 15 & 15 & 19 & 19 \\
\hline Min & -0.3 & 0.3 & -0.04 & 0.03 & 0.00 & 0.00 & 0.30 & 0.10 & -0.002 & 0.000 & -0.006 & 0.000 & 0.001 & 0.001 & 0.7 & 0.5 & 1.2 & 0.0 & -4.1 & 0.0 \\
\hline $\operatorname{Max}$ & 1.0 & 1.0 & 0.77 & 0.20 & 0.56 & 0.63 & 4.60 & 1.20 & 0.011 & 0.030 & 0.011 & 0.020 & 0.003 & 0.030 & 8.0 & 2.1 & 7.2 & 1.2 & 8.0 & 1.0 \\
\hline$(x+2 s)$ & 1.1 & & 0.60 & & 0.39 & & 4.02 & & 0.007 & & 0.008 & & 0.004 & & 7.2 & & 6.4 & & 7.5 & \\
\hline$(x+5 s)$ & 2.5 & & 1.28 & & 0.74 & & 6.83 & & 0.016 & & 0.016 & & 0.007 & & 13.1 & & 11.5 & & 15.4 & \\
\hline BGUL (95\%) & 2.7 & & 0.77 & & 0.54 & & 4.60 & & 0.010 & & 0.010 & & 0.003 & & 8.0 & & 7.2 & & 8.0 & \\
\hline \multicolumn{21}{|l|}{ Notes: } \\
\hline
\end{tabular}


Appendix A. Radiochemical Analyses of River Sediments

\begin{tabular}{|c|c|c|c|c|c|c|c|c|c|c|c|c|c|c|c|c|c|c|c|c|}
\hline \multicolumn{21}{|c|}{ Table A-5. Sediment Station: Rio Grande at Otowi Bridge } \\
\hline & & & & & & & & & & & & & & & Gross & & Gross & & Gross & \\
\hline $\begin{array}{c}\text { Sample } \\
\text { Date }\end{array}$ & $\begin{array}{c}{ }^{3} \mathbf{H} \\
\left(\mathrm{nCi}^{2} / \mathbf{)}\right) \\
\end{array}$ & $\pm \mathbf{u}$ & $\begin{array}{c}{ }^{90} \mathrm{Sr} \\
(\mathrm{pCi} / \mathrm{g})\end{array}$ & $\pm \mathbf{u}$ & $\begin{array}{c}{ }^{137} \mathrm{Cs} \\
(\mathrm{pCi} / \mathrm{g})\end{array}$ & $\pm \mathbf{u}$ & $\begin{array}{l}\text { Total U } \\
(\mathbf{m g} / \mathbf{k g})\end{array}$ & $\pm \mathbf{u}$ & $\begin{array}{c}{ }^{238} \mathrm{Pu} \\
(\mathrm{pCi} / \mathrm{g})\end{array}$ & $\pm \mathbf{u}$ & $\begin{array}{l}{ }^{239,240} \mathrm{Pu} \\
(\mathrm{pCi} / \mathrm{g})\end{array}$ & $\pm \mathbf{u}$ & $\begin{array}{r}{ }^{241} \mathrm{Am} \\
(\mathrm{pCi} / \mathrm{g})\end{array}$ & $\pm \mathbf{u}$ & $\begin{array}{c}\text { Alpha } \\
\text { (pCi/g) }\end{array}$ & $\pm \mathbf{u}$ & $\begin{array}{c}\text { Beta } \\
\text { (pCi/g) }\end{array}$ & $\pm \mathbf{u}$ & $\begin{array}{c}\text { Gamma } \\
(\mathrm{pCi} / \mathrm{g})\end{array}$ & $\pm \mathbf{u}$ \\
\hline $07 / 01 / 74$ & & & & & 0.16 & 0.12 & & & 0.000 & 0.000 & 0.032 & 0.008 & & & & & & & & \\
\hline $05 / 01 / 75$ & & & & & & & & & 0.000 & 0.000 & 0.013 & 0.003 & & & & & & & & \\
\hline 09/01/75 & & & & & & & & & -0.002 & 0.001 & 0.013 & 0.011 & & & & & & & & \\
\hline $04 / 29 / 76$ & 1.2 & 0.3 & 1.44 & 1.52 & 0.20 & 0.02 & 0.50 & 0.50 & -0.001 & 0.001 & 0.002 & 0.001 & & & 3.5 & 1.0 & 3.7 & 0.5 & & \\
\hline $10 / 04 / 76$ & & & & & & & & & 0.000 & 0.001 & 0.003 & 0.002 & & & 2.3 & 0.8 & 3.0 & 0.5 & & \\
\hline 03/08/77 & & & 0.08 & 0.04 & 0.06 & 0.03 & 0.50 & 0.70 & 0.003 & 0.003 & 0.005 & 0.003 & & & 1.7 & 0.5 & 2.3 & 0.4 & & \\
\hline $10 / 14 / 77$ & 1.0 & 0.3 & 0.51 & 0.10 & -0.08 & 0.08 & 1.30 & 0.50 & 0.000 & 0.001 & 0.003 & 0.002 & & & 2.3 & 0.6 & 3.4 & 0.5 & 2.1 & 0.1 \\
\hline $03 / 06 / 78$ & & & & & 0.08 & 0.02 & & & 0.000 & 0.001 & 0.000 & 0.001 & -0.040 & 0.040 & 1.4 & 0.4 & 0.9 & 0.3 & 0.1 & 0.1 \\
\hline $03 / 01 / 79$ & & & 0.12 & 0.09 & 0.11 & 0.09 & 2.70 & 0.30 & 0.000 & 0.000 & 0.007 & 0.000 & & & 3.1 & 0.8 & 5.0 & 0.7 & 3.0 & 0.1 \\
\hline $02 / 01 / 80$ & & & 0.42 & 0.08 & 0.09 & 0.05 & 2.60 & 0.20 & 0.001 & 0.001 & 0.000 & 0.001 & -0.003 & 0.003 & 3.3 & 0.8 & 3.2 & 0.5 & 2.8 & 0.1 \\
\hline 03/01/81 & & & 0.15 & 0.06 & 0.14 & 0.05 & & & 0.001 & 0.001 & 0.001 & 0.001 & & & 6.7 & 1.6 & 8.9 & 1.0 & 3.7 & 0.1 \\
\hline 03/01/82 & & & & & 0.16 & 0.03 & 2.40 & 0.20 & 0.002 & 0.001 & 0.065 & 0.005 & & & 1.9 & 1.0 & 2.3 & 0.8 & & \\
\hline $02 / 01 / 83$ & & & & & 0.20 & 0.05 & 3.00 & 0.20 & 0.003 & 0.002 & 0.003 & 0.001 & & & & & & & 3.9 & 0.2 \\
\hline $02 / 01 / 84$ & & & & & 0.18 & 0.15 & 2.70 & 0.10 & -0.001 & 0.004 & 0.002 & 0.004 & & & & & & & 4.3 & 0.6 \\
\hline $03 / 01 / 85$ & & & & & 0.12 & 0.08 & 2.60 & 0.20 & -0.001 & 0.001 & 0.003 & 0.002 & & & & & & & 2.3 & 0.4 \\
\hline $02 / 01 / 86$ & & & & & 0.09 & 0.07 & 4.40 & 0.40 & 0.001 & 0.001 & 0.003 & 0.001 & & & & & & & 5.2 & 0.4 \\
\hline 03/01/87 & & & & & 0.06 & 0.06 & 2.00 & 0.20 & 0.001 & 0.002 & 0.000 & 0.002 & -0.078 & 0.081 & & & & & -5.5 & 0.8 \\
\hline $03 / 01 / 88$ & & & & & 0.09 & 0.07 & 1.10 & 0.20 & 0.000 & 0.001 & 0.002 & 0.001 & & & & & & & -0.4 & 0.5 \\
\hline 03/01/89 & & & & & 0.28 & 0.13 & 1.20 & 0.20 & 0.000 & 0.001 & 0.000 & 0.001 & & & & & & & -0.5 & 0.4 \\
\hline 03/01/90 & & & & & 0.71 & 0.44 & & & 0.002 & 0.001 & 0.003 & 0.001 & & & & & & & 3.1 & 0.5 \\
\hline 03/01/91 & & & 0.20 & 0.20 & 0.10 & 0.10 & 2.40 & 0.20 & 0.002 & 0.001 & 0.002 & 0.001 & & & 3.0 & 1.0 & 2.0 & 0.0 & 1.0 & 0.0 \\
\hline $03 / 01 / 92$ & -0.2 & 0.3 & 0.10 & 0.20 & 0.30 & 0.20 & 1.10 & 0.10 & 0.005 & 0.003 & 0.003 & 0.002 & 0.052 & 0.069 & 1.0 & 0.0 & 1.0 & 0.0 & 5.0 & 1.0 \\
\hline 03/01/93 & 0.1 & 0.3 & & & 0.00 & 0.02 & 0.80 & 0.10 & 0.001 & 0.030 & 0.003 & 0.020 & & & & & & & & \\
\hline 03/01/94 & 0.0 & 0.3 & 0.20 & 0.30 & 7.70 & 0.66 & 1.50 & 0.40 & 0.000 & 0.030 & 0.001 & 0.020 & 0.004 & 0.030 & 2.0 & 0.0 & 1.0 & 0.0 & 0.0 & 0.0 \\
\hline $03 / 23 / 95$ & 0.2 & 0.4 & 0.00 & 0.20 & 0.03 & 0.02 & 1.57 & 0.28 & 0.009 & 0.002 & 0.024 & 0.003 & 0.004 & 0.001 & 1.3 & 0.3 & 1.4 & 0.2 & 1.7 & 0.2 \\
\hline $09 / 15 / 95$ & 0.0 & 0.3 & 0.20 & 0.20 & 0.07 & 0.02 & & & 0.002 & 0.001 & 0.003 & 0.001 & & & 3.0 & 0.6 & 1.4 & 0.2 & 3.0 & 0.3 \\
\hline $05 / 09 / 96$ & 0.0 & 1.0 & -0.10 & 0.20 & 0.05 & 0.02 & 1.10 & 0.11 & & & & & 0.000 & 0.001 & 1.5 & 0.7 & 2.4 & 0.3 & 1.9 & 0.3 \\
\hline $10 / 11 / 96$ & & & 1.80 & 0.08 & 0.09 & 0.03 & 4.17 & 0.42 & 0.001 & 0.000 & 0.004 & 0.001 & 0.003 & 0.001 & 6.3 & 1.9 & 5.0 & 0.6 & 3.0 & 0.4 \\
\hline \multicolumn{21}{|c|}{ Table A-6. Statistical Summary for Rio Grande at Otowi Bridge + A50 } \\
\hline & & & & & & & & & & & & & & & Gross & & Gross & & Gross & \\
\hline & ${ }^{3} \mathbf{H}$ & $\pm \mathbf{u}$ & ${ }^{90} \mathrm{Sr}$ & $\pm \mathbf{u}$ & ${ }^{137} \mathrm{Cs}$ & $\pm \mathbf{u}$ & Total U & $\pm \mathbf{u}$ & ${ }^{238} \mathbf{P u}$ & $\pm \mathbf{u}$ & ${ }^{239,240} \mathrm{Pu}$ & $\pm \mathbf{u}$ & ${ }^{241} \mathrm{Am}$ & $\pm \mathbf{u}$ & Alpha & $\pm \mathbf{u}$ & Beta & $\pm \mathbf{u}$ & Gamma & $\pm \mathbf{u}$ \\
\hline Statistic & $(\mathrm{nCi} / \mathbf{l})$ & & $(\mathrm{pCi} / \mathrm{g})$ & & $(\mathrm{pCi} / \mathrm{g})$ & & $(\mathrm{mg} / \mathrm{kg})$ & & $(\mathrm{pCi} / \mathrm{g})$ & & $(\mathrm{pCi} / \mathrm{g})$ & & $(\mathrm{pCi} / \mathrm{g})$ & & $(\mathrm{pCi} / \mathrm{g})$ & & $(\mathbf{p C i} / \mathrm{g})$ & & $(\mathrm{pCi} / \mathrm{g})$ & \\
\hline Mean (x) & 0.0 & 0.4 & 0.31 & 0.15 & 0.14 & 0.08 & 1.98 & 0.28 & 0.001 & 0.003 & 0.007 & 0.004 & 0.002 & 0.007 & 2.8 & 0.8 & 2.9 & 0.4 & 2.0 & 0.3 \\
\hline Median (m) & 0.0 & 0.3 & 0.18 & 0.15 & 0.10 & 0.06 & 1.79 & 0.20 & 0.001 & 0.001 & 0.003 & 0.002 & 0.003 & 0.001 & 2.3 & 0.8 & 2.4 & 0.5 & 2.6 & 0.3 \\
\hline Std Dev (s) & 0.1 & 0.3 & 0.50 & 0.08 & 0.15 & 0.09 & 1.11 & 0.16 & 0.002 & 0.008 & 0.014 & 0.005 & 0.003 & 0.013 & 1.6 & 0.5 & 2.1 & 0.3 & 2.4 & 0.3 \\
\hline Count & 6 & 6 & 12 & 12 & 24 & 24 & 20 & 20 & 27 & 27 & 27 & 27 & 5 & 5 & 16 & 16 & 16 & 16 & 20 & 20 \\
\hline Min & -0.2 & 0.3 & -0.10 & 0.04 & -0.08 & 0.02 & 0.50 & 0.10 & -0.002 & 0.000 & 0.000 & 0.000 & -0.003 & 0.001 & 1.0 & 0.0 & 0.9 & 0.0 & -5.5 & 0.0 \\
\hline Max & 0.2 & 1.0 & 1.80 & 0.30 & 0.71 & 0.44 & 4.40 & 0.70 & 0.009 & 0.030 & 0.065 & 0.020 & 0.004 & 0.030 & 6.7 & 1.9 & 8.9 & 1.0 & 5.2 & 1.0 \\
\hline$(x+2 s)$ & 0.3 & & 1.30 & & 0.43 & & 4.20 & & 0.005 & & 0.035 & & 0.008 & & 6.1 & & 7.0 & & 6.9 & \\
\hline$(x+5 s)$ & 0.7 & & 2.80 & & 0.88 & & 7.53 & & 0.012 & & 0.076 & & 0.017 & & 11.0 & & 13.2 & & 14.2 & \\
\hline BGUL (95\%) & 1.2 & & 1.80 & & 0.67 & & 4.40 & & 0.008 & & 0.059 & & 0.004 & & 6.7 & & 8.9 & & 5.2 & \\
\hline Notes: & (1) Individ & & ated an & & have ele & & ainities & & et labor & & contro & ific & nns. Data a & & in stat & al & mary. & & & \\
\hline
\end{tabular}


Appendix A. Radiochemical Analyses of River Sediments

\begin{tabular}{|c|c|c|c|c|c|c|c|c|c|c|c|c|c|c|c|c|c|c|c|c|}
\hline \multicolumn{21}{|c|}{ Table A-7. Sediment Station: Rio Grande below Sandia Canyon } \\
\hline & & & & & & & & & & & & & \multirow{3}{*}{$\begin{array}{l}{ }^{241} \mathrm{Am} \\
(\mathrm{pCi} / \mathrm{g})\end{array}$} & \multirow{3}{*}{$\pm \mathbf{u}$} & Gross & \multirow{3}{*}{$\pm \mathbf{u}$} & Gross & \multirow{3}{*}{$\pm \mathbf{u}$} & Gross & \multirow{3}{*}{$\pm \mathbf{u}$} \\
\hline Sample & ${ }^{3} \mathbf{H}$ & $\pm \mathbf{u}$ & ${ }^{9} \mathrm{Sr}$ & $\pm \mathbf{u}$ & ${ }^{137} \mathrm{Cs}$ & $\pm \mathbf{u}$ & Total U & $\pm \mathbf{u}$ & ${ }^{238} \mathbf{P u}$ & $\pm \mathbf{u}$ & ${ }^{239,240} \mathrm{Pu}$ & $\pm \mathbf{u}$ & & & Alpha & & Beta & & Gamma & \\
\hline Date & $(\mathrm{nCi} / \mathrm{l})$ & & $(\mathrm{pCi} / \mathrm{g})$ & & $(\mathrm{pCi} / \mathrm{g})$ & & $(\mathrm{mg} / \mathrm{kg})$ & & $(\mathrm{pCi} / \mathrm{g})$ & & $(\mathrm{pCi} / \mathrm{g})$ & & & & $(\mathrm{pCi} / \mathrm{g})$ & & $(\mathrm{pCi} / \mathrm{g})$ & & $(\mathrm{pCi} / \mathrm{g})$ & \\
\hline $09 / 01 / 78$ & & & & & 0.13 & 0.03 & & & -0.005 & 0.008 & -0.013 & 0.008 & & & & & & & & \\
\hline $11 / 01 / 79$ & & & -0.01 & 0.07 & 0.17 & 0.03 & 2.20 & 0.20 & 0.000 & 0.000 & 0.004 & 0.000 & & & 3.3 & 0.8 & 3.6 & 0.5 & 3.1 & 0.1 \\
\hline $10 / 01 / 80$ & & & & & 0.40 & 0.05 & 3.20 & 0.30 & 0.000 & 0.001 & 0.007 & 0.002 & & & 3.9 & 0.9 & 3.2 & 0.3 & 3.7 & 0.1 \\
\hline 10/01/81 & & & & & 0.21 & 0.03 & & & 0.000 & 0.000 & 0.002 & 0.001 & & & 3.5 & 0.9 & 6.9 & 0.8 & & \\
\hline 09/01/82 & & & & & 0.19 & 0.03 & 2.40 & 0.20 & 0.001 & 0.000 & 0.011 & 0.001 & & & & & & & & \\
\hline 09/01/83 & & & & & 0.15 & 0.05 & 2.80 & 0.50 & 0.001 & 0.003 & 0.006 & 0.003 & & & & & & & & \\
\hline 09/01/84 & & & & & 0.09 & 0.06 & 2.90 & 0.20 & -0.006 & 0.007 & -0.002 & 0.008 & & & & & & & & \\
\hline 09/01/85 & & & & & 0.23 & 0.08 & 3.30 & 0.20 & 0.000 & 0.001 & 0.002 & 0.001 & & & & & & & & \\
\hline $10 / 01 / 86$ & & & & & 0.17 & 0.07 & 2.90 & 0.30 & 0.000 & 0.001 & 0.003 & 0.001 & & & & & & & & \\
\hline 09/01/87 & 0.7 & 0.4 & & & 0.38 & 0.11 & 8.50 & 0.90 & 0.001 & 0.002 & 0.002 & 0.001 & & & & & & & & \\
\hline 09/01/88 & & & & & & & & & 0.002 & 0.001 & 0.003 & 0.001 & & & & & & & & \\
\hline 09/01/89 & & & & & -0.02 & 0.09 & 3.20 & 0.30 & 0.001 & 0.001 & 0.003 & 0.001 & & & & & & & & \\
\hline 09/01/90 & 0.3 & 0.3 & & & 0.24 & 0.10 & 2.70 & 0.30 & 0.000 & 0.001 & 0.003 & 0.001 & & & & & & & & \\
\hline 09/01/91 & -0.6 & 0.3 & 0.20 & 0.20 & 0.20 & 0.10 & 3.20 & 0.30 & 0.001 & 0.001 & 0.004 & 0.001 & & & 3.0 & 1.0 & 3.0 & 0.0 & 2.0 & 0.0 \\
\hline 09/01/92 & 0.3 & 0.3 & 0.20 & 0.20 & 0.10 & 0.10 & 2.10 & 0.10 & 0.000 & 0.003 & 0.003 & 0.002 & 0.001 & 0.003 & 2.0 & 1.0 & 3.0 & 0.0 & 3.0 & 1.0 \\
\hline $09 / 01 / 93$ & -0.1 & 0.3 & & & 0.10 & 0.00 & 2.50 & 0.30 & 0.007 & 0.030 & 0.003 & 0.020 & & & 7.0 & 2.0 & 4.0 & 1.0 & 3.0 & 0.0 \\
\hline 09/01/94 & -0.4 & 0.3 & 0.00 & 0.30 & 0.03 & 0.01 & 2.70 & 0.40 & 0.008 & 0.002 & 0.011 & 0.002 & 0.003 & 0.001 & 4.0 & 1.0 & 3.0 & 0.0 & 4.0 & 1.0 \\
\hline \multirow{2}{*}{\multicolumn{21}{|c|}{$\begin{array}{l}09 / 13 / 95 \\
03 / 11 / 96\end{array}$}} \\
\hline & & & & & & & & & & & & & & & & & & & & \\
\hline \multicolumn{21}{|c|}{ Table A-8. Statistical Summary for Rio Grande below Sandia Canyon } \\
\hline & & & & & & & & & & & \multirow{3}{*}{$\begin{array}{l}{ }^{23,240} \mathbf{P u} \\
(\mathrm{pCi} / \mathrm{g})\end{array}$} & & \multirow{3}{*}{$\begin{array}{l}{ }^{241} \mathrm{Am} \\
(\mathrm{pCi} / \mathrm{g})\end{array}$} & & Gross & \multirow{3}{*}{$\pm \mathbf{u}$} & Gross & & Gross & \multirow{3}{*}{$\pm \mathbf{u}$} \\
\hline & ${ }^{3} \mathbf{H}$ & $\pm \mathbf{u}$ & ${ }^{9} \mathrm{Sr}$ & $\pm \mathbf{u}$ & ${ }^{137} \mathrm{Cs}$ & $\pm \mathbf{u}$ & Total U & $\pm \mathbf{u}$ & ${ }^{238} \mathbf{P u}$ & $\pm \mathbf{u}$ & & $\pm \mathbf{u}$ & & $\pm \mathbf{u}$ & Alpha & & Beta & $\pm \mathbf{u}$ & Gamma & \\
\hline Statistic & $(\mathrm{nCi} / \mathbf{l})$ & & $(\mathrm{pCi} / \mathrm{g})$ & & $(\mathrm{pCi} / \mathrm{g})$ & & $(\mathrm{mg} / \mathrm{kg})$ & & $(\mathrm{pCi} / \mathrm{g})$ & & & & & & $(\mathrm{pCi} / \mathrm{g})$ & & $(\mathrm{pCi} / \mathrm{g})$ & & $(\mathrm{pCi} / \mathrm{g})$ & \\
\hline Mean (x) & 0.0 & 0.3 & 0.10 & 0.19 & 0.17 & 0.06 & 3.19 & 0.32 & 0.001 & 0.004 & 0.003 & 0.003 & 0.002 & 0.002 & 3.8 & 1.1 & 3.8 & 0.4 & 3.1 & 0.4 \\
\hline Median (m) & 0.1 & 0.3 & 0.10 & 0.20 & 0.17 & 0.06 & 2.85 & 0.30 & 0.000 & 0.001 & 0.003 & 0.001 & 0.002 & 0.002 & 3.5 & 1.0 & 3.2 & 0.3 & 3.1 & 0.1 \\
\hline Std Dev (s) & 0.5 & 0.0 & 0.12 & 0.10 & 0.11 & 0.04 & 1.58 & 0.19 & 0.003 & 0.007 & 0.005 & 0.005 & 0.001 & 0.001 & 1.6 & 0.4 & 1.4 & 0.4 & 0.7 & 0.5 \\
\hline Count & 6 & 6 & 4 & 4 & 16 & 16 & 14 & 14 & 17 & 17 & 17 & 17 & 2 & 2 & 7 & 7 & 7 & 7 & 6 & 6 \\
\hline Min & -0.6 & 0.3 & -0.01 & 0.07 & -0.02 & 0.00 & 2.10 & 0.10 & -0.006 & 0.000 & -0.013 & 0.000 & 0.001 & 0.001 & 2.0 & 0.8 & 3.0 & 0.0 & 2.0 & 0.0 \\
\hline Max & 0.7 & 0.4 & 0.20 & 0.30 & 0.40 & 0.11 & 8.50 & 0.90 & 0.008 & 0.030 & 0.011 & 0.020 & 0.003 & 0.003 & 7.0 & 2.0 & 6.9 & 1.0 & 4.0 & 1.0 \\
\hline$(x+2 s)$ & 1.0 & & 0.33 & & 0.39 & & 6.34 & & 0.007 & & 0.014 & & 0.005 & & 6.9 & & 6.6 & & 4.5 & \\
\hline$(x+5 s)$ & 2.5 & & 0.69 & & 0.72 & & 11.06 & & 0.017 & & 0.029 & & 0.009 & & 11.6 & & 10.9 & & 6.6 & \\
\hline BGUL (95\%) & 0.7 & & 0.20 & & 0.40 & & 8.50 & & 0.008 & & 0.011 & & 0.003 & & 7.0 & & 6.9 & & 4.0 & \\
\hline Notes: & $\begin{array}{l}\text { (1) Individu } \\
\text { (2) Radioac }\end{array}$ & hig & ted an & & $\begin{array}{l}\text { have ele } \\
\text { hown as }\end{array}$ & d un & $\begin{array}{l}\text { ainities } \\
\text { ard devi }\end{array}$ & no & pt for ${ }^{3} \mathrm{H}$ & $\begin{array}{l}\text { y QA } \\
\text { iich i }\end{array}$ & $\begin{array}{l}\text { C control } \\
\text { standard }\end{array}$ & cific & nns. Data a & ot ir & led in stat & & nary. & & & \\
\hline
\end{tabular}


Appendix A. Radiochemical Analyses of River Sediments

\begin{tabular}{|c|c|c|c|c|c|c|c|c|c|c|c|c|c|c|c|c|c|c|c|c|}
\hline \multicolumn{21}{|c|}{ Table A-9. Sediment Station: Rio Grande below Pajarito Canyon } \\
\hline & & & & & & & & & & & \multirow{3}{*}{$\begin{array}{l}{ }^{239,240} \mathrm{Pu} \\
(\mathrm{pCi} / \mathrm{g})\end{array}$} & \multirow{3}{*}{$\pm \mathbf{u}$} & \multirow{3}{*}{$\begin{array}{l}{ }^{241} \mathbf{A m} \\
(\mathrm{pCi} / \mathrm{g})\end{array}$} & \multirow{3}{*}{$\pm \mathbf{u}$} & \multirow{3}{*}{$\begin{array}{l}\text { Gross } \\
\text { Alpha } \\
\text { (pCi/g) }\end{array}$} & \multirow{3}{*}{$\pm \mathbf{u}$} & \multirow{3}{*}{$\begin{array}{c}\text { Beta } \\
(\mathrm{pCi} / \mathrm{g}) \\
\end{array}$} & \multirow{3}{*}{$\pm \mathbf{u}$} & \multirow{3}{*}{$\begin{array}{c}\text { Gross } \\
\text { Gamma } \\
(\mathrm{pCi} / \mathrm{g})\end{array}$} & \multirow{3}{*}{$\pm \mathbf{u}$} \\
\hline Sample & ${ }^{3} \mathbf{H}$ & $\pm \mathbf{u}$ & ${ }^{90} \mathrm{Sr}$ & $\pm \mathbf{u}$ & ${ }^{137} \mathrm{Cs}$ & $\pm \mathbf{u}$ & Total U & $\pm \mathbf{u}$ & ${ }^{238} \mathrm{Pu}$ & $\pm \mathbf{u}$ & & & & & & & & & & \\
\hline Date & $(\mathrm{nCi} / \mathrm{l})$ & & $(\mathrm{pCi} / \mathrm{g})$ & & $(\mathrm{pCi} / \mathrm{g})$ & & $(\mathrm{mg} / \mathrm{kg})$ & & $(\mathrm{pCi} / \mathrm{g})$ & & & & & & & & & & & \\
\hline $09 / 19 / 78$ & & & & & 0.07 & 0.03 & & & -0.005 & 0.008 & 0.009 & 0.007 & & & & & & & & \\
\hline $11 / 01 / 79$ & & & 0.01 & 0.11 & 0.08 & 0.05 & 1.90 & 0.20 & 0.000 & 0.000 & 0.001 & 0.000 & & & 2.5 & 0.7 & 2.5 & 0.4 & 1.5 & 0.1 \\
\hline 10/01/80 & & & & & 0.05 & 0.04 & 2.90 & 0.30 & 0.001 & 0.001 & 0.003 & 0.001 & & & 7.1 & 1.6 & 9.6 & 1.0 & 2.8 & 0.1 \\
\hline $10 / 01 / 81$ & & & & & 0.16 & 0.04 & & & 0.000 & 0.000 & 0.004 & 0.001 & & & 3.4 & 0.9 & 7.6 & 0.9 & & \\
\hline 09/01/82 & & & & & 0.08 & 0.06 & & & 0.023 & 0.015 & 0.210 & 0.130 & & & & & & & & \\
\hline 09/01/83 & & & & & 0.13 & 0.03 & 2.80 & 0.50 & -0.002 & 0.002 & 0.001 & 0.003 & & & & & & & & \\
\hline $09 / 01 / 84$ & & & & & 0.09 & 0.06 & 4.90 & 0.30 & -0.002 & 0.002 & 0.000 & 0.001 & & & & & & & & \\
\hline 09/01/85 & & & & & 0.14 & 0.08 & 1.00 & 0.10 & 0.001 & 0.001 & 0.000 & 0.001 & & & & & & & & \\
\hline $10 / 01 / 86$ & & & & & 0.20 & 0.10 & 3.00 & 0.30 & 0.002 & 0.002 & 0.002 & 0.002 & & & & & & & & \\
\hline 09/01/87 & 0.2 & 0.4 & & & 0.12 & 0.10 & 3.40 & 0.40 & 0.000 & 0.007 & 0.007 & 0.002 & & & & & & & & \\
\hline \multicolumn{21}{|l|}{ 09/01/88 } \\
\hline 09/01/89 & & & & & 0.15 & 0.06 & 3.20 & 0.30 & 0.000 & 0.001 & 0.006 & 0.001 & & & & & & & & \\
\hline 09/01/90 & 0.3 & 0.3 & & & 0.22 & 0.10 & 3.30 & 0.30 & 0.001 & 0.001 & 0.003 & 0.001 & & & & & & & & \\
\hline 09/01/91 & -0.5 & 0.3 & 0.10 & 0.20 & 0.20 & 0.10 & 2.60 & 0.30 & 0.008 & 0.001 & 0.001 & 0.001 & & & 2.0 & 1.0 & 2.0 & 0.0 & 1.0 & 0.0 \\
\hline 09/01/92 & 0.2 & 0.3 & 0.00 & 0.20 & 0.10 & 0.10 & 1.60 & 0.00 & 0.005 & 0.003 & 0.004 & 0.002 & 0.001 & 0.003 & 2.0 & 1.0 & 4.0 & 1.0 & 3.0 & 1.0 \\
\hline $09 / 01 / 93$ & 0.2 & 0.3 & & & 0.10 & 0.00 & 3.30 & 0.40 & 0.001 & 0.030 & 0.003 & 0.020 & & & 5.0 & 1.0 & 4.0 & 0.0 & 3.0 & 0.0 \\
\hline $09 / 01 / 94$ & 0.0 & 0.3 & 0.00 & 0.40 & 0.05 & 0.02 & 2.30 & 0.30 & 0.000 & 0.001 & 0.002 & 0.001 & 0.004 & 0.001 & 4.0 & 1.0 & 4.0 & 1.0 & 3.0 & 0.0 \\
\hline \multirow{2}{*}{\multicolumn{21}{|c|}{$\begin{array}{l}09 / 13 / 95 \\
05 / 09 / 96\end{array}$}} \\
\hline & & & & & & & & & & & & & & & & & & & & \\
\hline \multicolumn{21}{|c|}{ Table A-10. Statistical Summary for Rio Grande below Pajarito Canyon } \\
\hline & & & & & & & & & & & & & & & Gross & & Gross & & Gross & \\
\hline & ${ }^{3} \mathbf{H}$ & $\pm \mathbf{u}$ & ${ }^{90} \mathrm{Sr}$ & $\pm \mathbf{u}$ & ${ }^{137} \mathrm{Cs}$ & $\pm \mathbf{u}$ & Total U & $\pm \mathbf{u}$ & ${ }^{238} \mathrm{Pu}$ & $\pm \mathbf{u}$ & ${ }^{239,240} \mathrm{Pu}$ & $\pm \mathbf{u}$ & ${ }^{241} \mathbf{A m}$ & $\pm \mathbf{u}$ & Alpha & $\pm \mathbf{u}$ & Beta & $\pm \mathbf{u}$ & Gamma & $\pm \mathbf{u}$ \\
\hline Statistic & $(\mathrm{nCi} / \mathbf{l})$ & & $(\mathrm{pCi} / \mathrm{g})$ & & $(\mathrm{pCi} / \mathrm{g})$ & & $(\mathrm{mg} / \mathbf{k g})$ & & $(\mathrm{pCi} / \mathrm{g})$ & & $(\mathrm{pCi} / \mathrm{g})$ & & $(\mathrm{pCi} / \mathrm{g})$ & & $(\mathrm{pCi} / \mathrm{g})$ & & $(\mathrm{pCi} / \mathrm{g})$ & & $(\mathrm{pCi} / \mathrm{g})$ & \\
\hline Mean (x) & 0.1 & 0.3 & 0.03 & 0.23 & 0.12 & 0.06 & 2.78 & 0.28 & 0.002 & 0.005 & 0.003 & 0.003 & 0.003 & 0.002 & 3.7 & 1.0 & 4.8 & 0.6 & 2.4 & 0.2 \\
\hline Median (m) & 0.2 & 0.3 & 0.01 & 0.20 & 0.11 & 0.06 & 2.90 & 0.30 & 0.001 & 0.002 & 0.003 & 0.001 & 0.003 & 0.002 & 3.4 & 1.0 & 4.0 & 0.9 & 2.9 & 0.1 \\
\hline Std Dev (s) & 0.3 & 0.0 & 0.05 & 0.12 & 0.05 & 0.03 & 0.97 & 0.13 & 0.006 & 0.008 & 0.003 & 0.005 & 0.002 & 0.001 & 1.9 & 0.3 & 2.8 & 0.5 & 0.9 & 0.4 \\
\hline Count & 6 & 6 & 4 & 4 & 16 & 16 & 13 & 13 & 16 & 16 & 15 & 15 & 2 & 2 & 7 & 7 & 7 & 7 & 6 & 6 \\
\hline Min & -0.5 & 0.3 & 0.00 & 0.11 & 0.05 & 0.00 & 1.00 & 0.00 & -0.005 & 0.000 & 0.000 & 0.000 & 0.001 & 0.001 & 2.0 & 0.7 & 2.0 & 0.0 & 1.0 & 0.0 \\
\hline Max & 0.3 & 0.4 & 0.10 & 0.40 & 0.22 & 0.10 & 4.90 & 0.50 & 0.023 & 0.030 & 0.009 & 0.020 & 0.004 & 0.003 & 7.1 & 1.6 & 9.6 & 1.0 & 3.0 & 1.0 \\
\hline$(x+2 s)$ & 0.7 & & 0.12 & & 0.23 & & 4.72 & & 0.015 & & 0.008 & & 0.007 & & 7.4 & & 10.3 & & 4.2 & \\
\hline$(x+5 s)$ & 1.5 & & 0.27 & & 0.39 & & 7.63 & & 0.033 & & 0.016 & & 0.013 & & 13.0 & & 18.6 & & 6.9 & \\
\hline BGUL (95\%) & 0.3 & & 0.10 & & 0.22 & & 4.90 & & 0.023 & & 0.009 & & 0.004 & & 7.1 & & 9.6 & & 3.0 & \\
\hline Notes: & $\begin{array}{l}\text { (1) Individu } \\
\text { (2) Radioac }\end{array}$ & & We anc & & 年 & & mintes & & 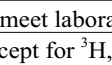 & & andarc & 1116 & 1S. Data & & In sta & & mary. & & & \\
\hline
\end{tabular}


Appendix A. Radiochemical Analyses of River Sediments

\begin{tabular}{|c|c|c|c|c|c|c|c|c|c|c|c|c|c|c|c|c|c|c|c|c|}
\hline \multicolumn{21}{|c|}{ Table A-11. Sediment Station: Rio Grande below Ancho Canyon } \\
\hline & & & & & & & & & & & & & & & Gross & & Gross & & Gross & \\
\hline Sample & ${ }^{3} \mathbf{H}$ & $\pm \mathbf{u}$ & ${ }^{90} \mathrm{Sr}$ & $\pm \mathbf{u}$ & ${ }^{137} \mathrm{Cs}$ & $\pm \mathbf{u}$ & Total U & $\pm \mathbf{u}$ & ${ }^{238} \mathrm{Pu}$ & $\pm \mathbf{u}$ & ${ }^{239,240} \mathbf{P u}$ & $\pm \mathbf{u}$ & ${ }^{241} \mathrm{Am}$ & $\pm \mathbf{u}$ & Alpha & $\pm \mathbf{u}$ & Beta & $\pm \mathbf{u}$ & Gamma & $\pm \mathbf{u}$ \\
\hline Date & $(\mathrm{nCi} / \mathrm{l})$ & & $(\mathrm{pCi} / \mathrm{g})$ & & $(\mathrm{pCi} / \mathrm{g})$ & & $(\mathrm{mg} / \mathbf{k g})$ & & $(\mathrm{pCi} / \mathrm{g})$ & & $(\mathrm{pCi} / \mathrm{g})$ & & $(\mathrm{pCi} / \mathrm{g})$ & & $(\mathrm{pCi} / \mathrm{g})$ & & $(\mathrm{pCi} / \mathrm{g})$ & & $(\mathrm{pCi} / \mathrm{g})$ & \\
\hline $09 / 20 / 78$ & & & & & 0.13 & 0.03 & & & -0.006 & 0.013 & -0.003 & 0.010 & & & & & & & & \\
\hline 11/01/79 & & & 2.50 & 0.20 & 0.32 & 0.05 & 2.90 & 0.20 & 0.001 & 0.000 & 0.009 & 0.000 & & & 5.3 & 1.3 & 6.6 & 0.8 & 4.3 & 0.1 \\
\hline 10/01/80 & & & & & 0.30 & 0.04 & 2.70 & 0.20 & -0.001 & 0.002 & 0.003 & 0.002 & & & 7.8 & 1.7 & 11.1 & 1.2 & 2.9 & 0.1 \\
\hline 10/01/81 & & & & & 0.23 & 0.07 & & & 0.001 & 0.001 & 0.002 & 0.001 & & & 5.5 & 0.4 & 8.5 & 1.0 & & \\
\hline 09/01/82 & & & 0.13 & 0.05 & 0.16 & 0.04 & & & 0.000 & 0.000 & 0.015 & 0.001 & & & & & & & & \\
\hline 09/01/83 & & & & & 0.04 & 0.06 & 1.60 & 0.50 & -0.003 & 0.004 & -0.001 & 0.002 & & & & & & & & \\
\hline 09/01/84 & & & & & 0.26 & 0.05 & 1.70 & 0.10 & -0.001 & 0.006 & -0.004 & 0.004 & & & & & & & & \\
\hline $09 / 01 / 85$ & & & & & 0.53 & 0.13 & 1.40 & 0.10 & -0.002 & 0.002 & 0.019 & 0.004 & & & & & & & & \\
\hline $10 / 01 / 86$ & & & & & 0.13 & 0.07 & 2.50 & 0.30 & -0.002 & 0.001 & 0.002 & 0.002 & & & & & & & & \\
\hline 09/01/87 & & & & & & & & & 0.000 & 0.001 & 0.000 & 0.001 & & & & & & & & \\
\hline 09/01/88 & & & & & & & & & -0.004 & 0.001 & 0.004 & 0.001 & & & & & & & & \\
\hline 09/01/89 & & & & & -0.01 & 0.06 & 2.50 & 0.20 & 0.000 & 0.001 & 0.005 & 0.001 & & & & & & & & \\
\hline 09/01/90 & 0.3 & 0.3 & & & 0.22 & 0.08 & 2.50 & 0.30 & 0.000 & 0.001 & 0.004 & 0.001 & & & & & & & & \\
\hline 09/01/91 & -0.6 & 0.3 & 0.20 & 0.20 & 0.30 & 0.10 & 2.20 & 0.20 & 0.002 & 0.001 & 0.001 & 0.001 & & & 2.0 & 1.0 & 2.0 & 0.0 & 1.0 & 0.0 \\
\hline $09 / 01 / 92$ & 0.6 & 0.3 & 0.00 & 0.20 & 0.10 & 0.10 & 1.20 & 0.00 & 0.002 & 0.003 & 0.002 & 0.002 & 0.002 & 0.003 & 2.0 & 1.0 & 2.0 & 0.0 & 0.0 & 1.0 \\
\hline $09 / 01 / 93$ & -0.1 & 0.3 & & & 0.10 & 0.00 & 2.00 & 0.20 & 0.013 & 0.030 & 0.003 & 0.020 & & & 3.0 & 1.0 & 2.0 & 0.0 & 2.0 & 0.0 \\
\hline 09/01/94 & -0.4 & 0.3 & 0.00 & 4.10 & 0.08 & 0.03 & 2.30 & 0.50 & 0.004 & 0.001 & 0.004 & 0.001 & 0.004 & 0.001 & 4.0 & 1.0 & 4.0 & 1.0 & 3.0 & 0.0 \\
\hline \multirow{2}{*}{\multicolumn{21}{|c|}{$\begin{array}{l}09 / 13 / 95 \\
05 / 09 / 96\end{array}$}} \\
\hline & & & & & & & & & & & & & & & & & & & & \\
\hline \multicolumn{21}{|c|}{ Table A-12. Statistical Summary for Rio Grande below Ancho Canyon } \\
\hline & & & & & & & & & & & & & & & Gross & & Gross & & Gross & \\
\hline & ${ }^{3} \mathbf{H}$ & $\pm \mathbf{u}$ & ${ }^{90} \mathrm{Sr}$ & $\pm \mathbf{u}$ & ${ }^{137} \mathrm{Cs}$ & $\pm \mathbf{u}$ & Total U & $\pm \mathbf{u}$ & ${ }^{238} \mathbf{P u}$ & $\pm \mathbf{u}$ & ${ }^{239,240} \mathrm{Pu}$ & $\pm \mathbf{u}$ & ${ }^{241} \mathrm{Am}$ & $\pm \mathbf{u}$ & Alpha & $\pm \mathbf{u}$ & Beta & $\pm \mathbf{u}$ & Gamma & $\pm \mathbf{u}$ \\
\hline Statistic & $(\mathrm{nCi} / \mathrm{l})$ & & $(\mathrm{pCi} / \mathrm{g})$ & & $(\mathrm{pCi} / \mathrm{g})$ & & $(\mathrm{mg} / \mathbf{k g})$ & & $(\mathrm{pCi} / \mathrm{g})$ & & $(\mathrm{pCi} / \mathrm{g})$ & & $(\mathrm{pCi} / \mathrm{g})$ & & $(\mathrm{pCi} / \mathrm{g})$ & & $(\mathrm{pCi} / \mathrm{g})$ & & $(\mathrm{pCi} / \mathrm{g})$ & \\
\hline Mean (x) & 0.0 & 0.3 & 0.71 & 0.16 & 0.19 & 0.06 & 2.13 & 0.23 & 0.000 & 0.004 & 0.004 & 0.003 & 0.003 & 0.002 & 4.2 & 1.1 & 5.2 & 0.6 & 2.2 & 0.2 \\
\hline Median (m) & -0.1 & 0.3 & 0.17 & 0.20 & 0.16 & 0.06 & 2.25 & 0.20 & 0.000 & 0.001 & 0.003 & 0.001 & 0.003 & 0.002 & 4.0 & 1.0 & 4.0 & 0.8 & 2.4 & 0.1 \\
\hline Std Dev (s) & 0.5 & 0.0 & 1.20 & 0.08 & 0.14 & 0.03 & 0.54 & 0.15 & 0.004 & 0.007 & 0.006 & 0.005 & 0.001 & 0.001 & 2.1 & 0.4 & 3.6 & 0.5 & 1.5 & 0.4 \\
\hline Count & 5 & 5 & 4 & 4 & 15 & 15 & 12 & 12 & 17 & 17 & 17 & 17 & 2 & 2 & 7 & 7 & 7 & 7 & 6 & 6 \\
\hline Min & -0.6 & 0.3 & 0.00 & 0.05 & -0.01 & 0.00 & 1.20 & 0.00 & -0.006 & 0.000 & -0.004 & 0.000 & 0.002 & 0.001 & 2.0 & 0.4 & 2.0 & 0.0 & 0.0 & 0.0 \\
\hline Max & 0.6 & 0.3 & 2.50 & 0.20 & 0.53 & 0.13 & 2.90 & 0.50 & 0.013 & 0.030 & 0.019 & 0.020 & 0.004 & 0.003 & 7.8 & 1.7 & 11.1 & 1.2 & 4.3 & 1.0 \\
\hline$(x+2 s)$ & 0.9 & & 3.10 & & 0.47 & & 3.21 & & 0.008 & & 0.016 & & 0.006 & & 8.5 & & 12.5 & & 5.3 & \\
\hline$(x+5 s)$ & 2.4 & & 6.70 & & 0.88 & & 4.84 & & 0.021 & & 0.033 & & 0.010 & & 14.8 & & 23.4 & & 9.9 & \\
\hline BGUL (95\%) & 0.6 & & 2.50 & & 0.53 & & 2.90 & & 0.013 & & 0.019 & & 0.040 & & 7.8 & & 11.1 & & 4.3 & \\
\hline \multicolumn{21}{|l|}{ Notes: } \\
\hline
\end{tabular}


Appendix A. Radiochemical Analyses of River Sediments

\begin{tabular}{|c|c|c|c|c|c|c|c|c|c|c|c|c|c|c|c|c|c|c|c|c|}
\hline \multicolumn{21}{|c|}{ Table A-13. Sediment Station: Rio Grande below Frijoles Canyon } \\
\hline & & & & & & & & & & & \multirow{3}{*}{$\begin{array}{l}{ }^{239,240} \mathrm{Pu} \\
(\mathrm{pCi} / \mathrm{g})\end{array}$} & & \multirow{3}{*}{$\begin{array}{c}{ }^{241} \mathrm{Am} \\
(\mathrm{pCi} / \mathrm{g})\end{array}$} & \multirow[b]{2}{*}{$\pm \mathbf{u}$} & Gross & & \multirow{2}{*}{$\begin{array}{c}\text { Gross } \\
\text { Beta }\end{array}$} & \multirow{3}{*}{$\pm \mathbf{u}$} & \multirow{3}{*}{$\begin{array}{c}\text { Gross } \\
\text { Gamma } \\
\text { (pCi/g) }\end{array}$} & \multirow{3}{*}{$\pm \mathbf{u}$} \\
\hline Sample & ${ }^{3} \mathbf{H}$ & $\pm \mathbf{u}$ & ${ }^{90} \mathrm{Sr}$ & $\pm \mathbf{u}$ & ${ }^{137} \mathrm{Cs}$ & $\pm \mathbf{u}$ & \multirow{2}{*}{$\begin{array}{l}\text { Total U } \\
(\mathbf{m g} / \mathbf{k g})\end{array}$} & \multirow[t]{2}{*}{$\pm \mathbf{u}$} & \multirow{2}{*}{$\begin{array}{c}{ }^{238} \mathrm{Pu} \\
(\mathrm{pCi} / \mathrm{g}) \\
\end{array}$} & \multirow[t]{2}{*}{$\pm \mathbf{u}$} & & \multirow{2}{*}{$\pm \mathbf{u}$} & & & \multirow{2}{*}{$\begin{array}{l}\text { Alpha } \\
\text { (pCi/g) }\end{array}$} & \multirow{2}{*}{$\pm \mathbf{u}$} & & & & \\
\hline Date & $(\mathrm{nCi} / \mathrm{l})$ & & $(\mathrm{pCi} / \mathrm{g})$ & & $(\mathrm{pCi} / \mathrm{g})$ & & & & & & & & & & & & $(\mathrm{pCi} / \mathrm{g})$ & & & \\
\hline $03 / 01 / 76$ & 0.8 & 0.3 & 0.99 & 1.00 & 0.16 & 0.03 & 2.40 & 0.40 & 0.000 & 0.001 & 0.002 & 0.001 & & & 3.6 & 0.8 & 3.5 & 0.5 & & \\
\hline $10 / 01 / 76$ & & & 0.10 & 0.03 & & & & & 0.000 & 0.001 & 0.003 & 0.001 & & & 1.4 & 0.5 & 2.9 & 0.4 & & \\
\hline 03/09/77 & & & 0.04 & 0.01 & -0.14 & 0.07 & 1.40 & 0.90 & 0.005 & 0.006 & 0.001 & 0.001 & & & 1.3 & 0.4 & 2.4 & 0.4 & & \\
\hline $10 / \mathbf{1 4} / 77$ & 2.2 & 0.3 & 0.14 & 0.11 & 0.10 & 0.03 & 0.30 & 0.60 & 0.001 & 0.001 & 0.002 & 0.001 & & & 1.8 & 0.5 & 1.1 & 0.4 & 1.7 & 0.1 \\
\hline 09/01/78 & & & & & 0.15 & 0.03 & & & -0.012 & 0.010 & -0.003 & 0.010 & & & & & & & & \\
\hline $11 / 01 / 79$ & & & -0.16 & 0.10 & 0.12 & 0.03 & 2.20 & 0.20 & 0.000 & 0.000 & 0.002 & 0.002 & & & 3.9 & 0.9 & 2.6 & 0.5 & 2.1 & 0.1 \\
\hline $10 / 01 / 80$ & & & & & 0.10 & 0.05 & 2.60 & 0.30 & -0.001 & 0.001 & 0.004 & 0.002 & & & 3.2 & 0.8 & 4.4 & 0.5 & 2.0 & 0.1 \\
\hline $10 / 01 / 81$ & & & & & 0.20 & 0.07 & & & 0.000 & 0.000 & 0.002 & 0.001 & & & 2.1 & 0.1 & 4.1 & 0.6 & & \\
\hline 09/01/82 & & & 0.27 & 0.05 & 0.21 & 0.07 & & & 0.007 & 0.001 & 0.093 & 0.017 & & & 4.1 & 1.0 & & & & \\
\hline 09/01/83 & & & & & 0.09 & 0.02 & 2.00 & 0.50 & -0.003 & 0.003 & 0.003 & 0.003 & & & & & & & 2.2 & 0.1 \\
\hline 09/01/84 & & & & & 0.04 & 0.04 & 1.90 & 0.10 & -0.002 & 0.004 & -0.003 & 0.004 & & & & & & & 1.5 & 0.6 \\
\hline 09/01/85 & & & & & & & & & 0.000 & 0.001 & 0.004 & 0.001 & & & & & & & & \\
\hline 10/01/86 & & & & & 0.28 & 0.10 & 3.60 & 0.40 & 0.001 & 0.001 & 0.009 & 0.003 & & & & & & & 4.7 & 0.6 \\
\hline 09/01/87 & & & & & & & & & -0.001 & 0.001 & 0.002 & 0.001 & & & & & & & & \\
\hline \multicolumn{21}{|l|}{ 09/01/88 } \\
\hline 09/01/89 & & & & & -0.05 & 0.06 & 3.20 & 0.30 & 0.000 & 0.001 & 0.003 & 0.001 & & & & & & & 2.6 & 0.4 \\
\hline 09/01/90 & 0.4 & 0.3 & & & -0.14 & 0.10 & 3.20 & 0.30 & 0.000 & 0.001 & 0.002 & 0.001 & & & & & & & 2.4 & 0.5 \\
\hline 09/01/91 & -0.5 & 0.3 & 0.00 & 0.20 & 0.20 & 0.10 & 4.10 & 0.40 & 0.002 & 0.001 & 0.004 & 0.001 & & & 3.0 & 1.0 & 4.0 & 0.0 & 2.0 & 0.0 \\
\hline 09/01/92 & 0.2 & 0.3 & 0.00 & 0.20 & 0.20 & 0.10 & 1.70 & 0.00 & 0.003 & 0.003 & 0.009 & 0.002 & 0.002 & 0.003 & 3.0 & 1.0 & 4.0 & 0.0 & 3.0 & 1.0 \\
\hline 09/01/93 & 0.2 & 0.3 & & & 0.10 & 0.00 & 14.00 & 3.50 & 0.011 & 0.030 & 0.007 & 0.020 & & & 6.0 & 1.0 & 4.0 & 0.0 & 3.0 & 0.0 \\
\hline 09/01/94 & 0.3 & 0.3 & 0.40 & 0.30 & 0.04 & & 1.90 & 0.30 & 0.005 & 0.001 & 0.005 & 0.001 & 0.008 & 0.003 & 2.0 & 1.0 & 1.0 & 0.0 & 3.0 & 0.0 \\
\hline 09/13/95 & -0.1 & 0.3 & & & 0.03 & 0.01 & 1.11 & 0.11 & 0.002 & 0.001 & 0.004 & 0.001 & 0.001 & 0.001 & 1.0 & 0.2 & 0.5 & 0.1 & 2.0 & 0.3 \\
\hline $10 / 09 / 96$ & 8.0 & 49.0 & 1.40 & 0.50 & 0.09 & 0.03 & 2.89 & 0.29 & 0.000 & 0.000 & 0.003 & 0.001 & 0.003 & 0.001 & & & & & 3.0 & 0.4 \\
\hline $09 / 30 / 97$ & -0.1 & 0.7 & 0.73 & 1.26 & 0.06 & 0.01 & 2.61 & 0.26 & 0.001 & 0.001 & 0.002 & 0.001 & 0.004 & 0.001 & 3.8 & 2.0 & 3.6 & 0.9 & 2.1 & 0.2 \\
\hline \multicolumn{21}{|c|}{ Table A-14. Statistical Summary for Rio Grande below Frijoles Canyon } \\
\hline & & & & & & & & & & & & & & & Gross & & Gross & & Gross & \\
\hline & ${ }^{3} \mathbf{H}$ & $\pm \mathbf{u}$ & ${ }^{90} \mathrm{Sr}$ & $\pm \mathbf{u}$ & ${ }^{137} \mathrm{Cs}$ & $\pm \mathbf{u}$ & Total U & $\pm \mathbf{u}$ & ${ }^{238} \mathrm{Pu}$ & $\pm \mathbf{u}$ & ${ }^{239,240} \mathrm{Pu}$ & $\pm \mathbf{u}$ & ${ }^{241} \mathbf{A m}$ & $\pm \mathbf{u}$ & Alpha & $\pm \mathbf{u}$ & Beta & $\pm \mathbf{u}$ & Gamma & $\pm \mathbf{u}$ \\
\hline Statistic & $(\mathrm{nCi} / \mathrm{l})$ & & $(\mathrm{pCi} / \mathrm{g})$ & & $(\mathrm{pCi} / \mathrm{g})$ & & $(\mathrm{mg} / \mathrm{kg})$ & & $(\mathrm{pCi} / \mathrm{g})$ & & $(\mathrm{pCi} / \mathrm{g})$ & & $(\mathrm{pCi} / \mathrm{g})$ & & $(\mathrm{pCi} / \mathrm{g})$ & & $(\mathrm{pCi} / \mathrm{g})$ & & $(\mathrm{pCi} / \mathrm{g})$ & \\
\hline Mean (x) & 0.1 & 0.4 & 0.24 & 0.17 & 0.09 & 0.05 & 2.32 & 0.34 & 0.001 & 0.003 & 0.007 & 0.003 & 0.004 & 0.002 & 2.9 & 0.8 & 2.9 & 0.3 & 2.5 & 0.3 \\
\hline Median (m) & 0.2 & 0.3 & 0.10 & 0.11 & 0.10 & 0.04 & 2.30 & 0.30 & 0.000 & 0.001 & 0.003 & 0.001 & 0.003 & 0.001 & 3.0 & 0.9 & 3.5 & 0.4 & 2.2 & 0.2 \\
\hline Std Dev (s) & 0.3 & 0.2 & 0.46 & 0.16 & 0.11 & 0.03 & 0.97 & 0.21 & 0.004 & 0.006 & 0.019 & 0.005 & 0.003 & 0.001 & 1.4 & 0.5 & 1.3 & 0.3 & 0.8 & 0.3 \\
\hline Count & 7 & 7 & 9 & 9 & 20 & 19 & 16 & 16 & 23 & 23 & 23 & 23 & 5 & 5 & 14 & 14 & 13 & 13 & 15 & 15 \\
\hline Min & -0.5 & 0.3 & -0.16 & 0.01 & -0.14 & 0.00 & 0.30 & 0.00 & -0.012 & 0.000 & -0.003 & 0.001 & 0.001 & 0.001 & 1.0 & 0.1 & 0.5 & 0.0 & 1.5 & 0.0 \\
\hline Max & 0.4 & 0.7 & 1.40 & 0.50 & 0.28 & 0.10 & 4.10 & 0.90 & 0.011 & 0.030 & 0.093 & 0.020 & 0.008 & 0.003 & 6.0 & 2.0 & 4.4 & 0.9 & 4.7 & 1.0 \\
\hline$(x+2 s)$ & 0.7 & & 1.17 & & 0.31 & & 4.26 & & 0.009 & & 0.045 & & 0.009 & & 5.6 & & 5.6 & & 4.1 & \\
\hline$(x+5 s)$ & 1.6 & & 2.56 & & 0.64 & & 7.17 & & 0.022 & & 0.102 & & 0.017 & & 9.7 & & 9.6 & & 6.4 & \\
\hline BGUL (95\%) & 2.2 & & 1.40 & & 0.28 & & 4.10 & & 0.011 & & 0.087 & & 0.008 & & 6.0 & & 4.4 & & 4.7 & \\
\hline Notes: & ) Individ & & shted ana & & have ele & & tainities & & meet labor & & QC control & cificat & ions. Data a & ot in & ed in sta & sal s & Imary. & & & \\
\hline & Kadioac & & uncer & & nown & & ard dev & & t for ${ }^{3} \mathrm{H}$ & $\mathrm{ch}$ is & tandard & tion & & & & & & & & \\
\hline
\end{tabular}


Appendix A. Radiochemical Analyses of River Sediments

\begin{tabular}{|c|c|c|c|c|c|c|c|c|c|c|c|c|c|c|c|c|c|c|c|c|}
\hline \multicolumn{21}{|c|}{ Table A-15. Sediment Station: Rio Grande below Cochiti Reservoir Spillway } \\
\hline & & & & & & & & & & & & & \multirow{3}{*}{$\begin{array}{c}{ }^{241} \mathrm{Am} \\
(\mathrm{pCi} / \mathrm{g})\end{array}$} & \multirow{3}{*}{$\pm \mathbf{u}$} & Gross & & \multirow{3}{*}{$\begin{array}{c}\text { Gross } \\
\text { Beta } \\
(\mathrm{pCi} / \mathrm{g}) \\
\end{array}$} & \multirow{3}{*}{$\pm \mathbf{u}$} & Gross & \multirow{3}{*}{$\pm \mathbf{u}$} \\
\hline Sample & ${ }^{3} \mathbf{H}$ & $\pm \mathbf{u}$ & ${ }^{90} \mathrm{Sr}$ & $\pm \mathbf{u}$ & ${ }^{137} \mathrm{Cs}$ & $\pm \mathbf{u}$ & Total U & $\pm \mathbf{u}$ & ${ }^{238} \mathrm{Pu}$ & $\pm \mathbf{u}$ & \multirow{2}{*}{$\begin{array}{l}{ }^{239,240} \mathrm{Pu} \\
(\mathrm{pCi} / \mathrm{g})\end{array}$} & $\pm \mathbf{u}$ & & & Alpha & $\pm \mathbf{u}$ & & & \multirow{2}{*}{$\begin{array}{c}\text { Gamma } \\
(\mathrm{pCi} / \mathrm{g})\end{array}$} & \\
\hline Date & $(\mathrm{nCi} / \mathrm{l})$ & & $(\mathrm{pCi} / \mathrm{g})$ & & $(\mathrm{pCi} / \mathrm{g})$ & & $(\mathrm{mg} / \mathbf{k g})$ & & $(\mathrm{pCi} / \mathrm{g})$ & & & & & & $(\mathrm{pCi} / \mathrm{g})$ & & & & & \\
\hline 07/01/74 & & & & & 0.03 & 0.12 & & & 0.000 & 0.000 & 0.000 & 0.000 & & & & & & & & \\
\hline 05/01/75 & & & & & & & 0.80 & 0.10 & 0.000 & 0.000 & -0.001 & 0.001 & & & & & & & & \\
\hline $10 / 06 / 76$ & & & 0.90 & 0.30 & & & & & 0.003 & 0.002 & 0.002 & 0.001 & & & 0.5 & 0.4 & 1.7 & 0.4 & & \\
\hline 03/08/77 & & & 0.03 & 0.01 & 0.05 & 0.02 & 2.70 & 1.00 & -0.020 & 0.030 & -0.030 & 0.020 & & & 3.4 & 0.9 & 2.0 & 0.4 & & \\
\hline 10/19/77 & 3.4 & 0.4 & -0.05 & 0.09 & 0.04 & 0.02 & 1.10 & 0.50 & 0.000 & 0.001 & 0.001 & 0.001 & & & 2.0 & 0.5 & 1.3 & 0.4 & 1.0 & 0.1 \\
\hline $03 / 07 / 78$ & & & & & 0.03 & 0.05 & & & -0.002 & 0.002 & 0.001 & 0.002 & & & 1.5 & 0.4 & 1.5 & 0.4 & 0.9 & 0.1 \\
\hline $11 / 01 / 79$ & & & & & 0.29 & 0.05 & 2.90 & 0.30 & 0.001 & 0.002 & 0.032 & 0.005 & & & 13.0 & 3.0 & 14.0 & 1.6 & 3.1 & 0.1 \\
\hline $03 / 23 / 95$ & 0.2 & 0.3 & 0.30 & 0.20 & 0.08 & 0.03 & 1.81 & 0.25 & 0.007 & 0.001 & 0.008 & 0.001 & 0.001 & 0.001 & 2.0 & 0.5 & 2.3 & 0.3 & 2.2 & 0.3 \\
\hline \multicolumn{21}{|c|}{ Table A-16. Statistical Summary for Rio Grande below Cochiti Reservoir Spillway } \\
\hline & & & & & & & & & & & \multirow{3}{*}{$\begin{array}{l}{ }^{239,240} \mathbf{P u} \\
(\mathrm{pCi} / \mathrm{g}) \\
\end{array}$} & & \multirow{3}{*}{$\begin{array}{l}{ }^{241} \mathbf{A m} \\
(\mathrm{pCi} / \mathrm{g})\end{array}$} & & Gross & & \multirow{3}{*}{$\begin{array}{c}\text { Gross } \\
\text { Beta } \\
(\mathrm{pCi} / \mathrm{g})\end{array}$} & & \multirow{3}{*}{$\begin{array}{c}\text { Gross } \\
\text { Gamma } \\
(\mathrm{pCi} / \mathrm{g})\end{array}$} & \multirow{3}{*}{$\pm \mathbf{u}$} \\
\hline & ${ }^{3} \mathrm{H}$ & $\pm \mathbf{u}$ & ${ }^{90} \mathrm{Sr}$ & $\pm \mathbf{u}$ & ${ }^{137} \mathrm{Cs}$ & $\pm \mathbf{u}$ & Total U & $\pm \mathbf{u}$ & ${ }^{238} \mathbf{P u}$ & $\pm \mathbf{u}$ & & $\pm \mathbf{u}$ & & $\pm \mathbf{u}$ & Alpha & $\pm \mathbf{u}$ & & $\pm \mathbf{u}$ & & \\
\hline Statistic & $(\mathrm{nCi} / \mathrm{l})$ & & $(\mathrm{pCi} / \mathrm{g})$ & & $(\mathrm{pCi} / \mathrm{g})$ & & $(\mathrm{mg} / \mathrm{kg})$ & & $(\mathrm{pCi} / \mathrm{g})$ & & & & & & $(\mathrm{pCi} / \mathrm{g})$ & & & & & \\
\hline Mean (x) & & & 0.09 & 0.10 & 0.09 & 0.05 & 1.86 & 0.43 & -0.001 & 0.005 & 0.002 & 0.004 & 0.001 & 0.001 & 3.7 & 1.0 & 3.8 & 0.6 & 1.8 & 0.2 \\
\hline Median (m) & & & 0.03 & 0.09 & 0.05 & 0.04 & 1.81 & 0.30 & 0.000 & 0.002 & 0.001 & 0.001 & 0.001 & 0.001 & 2.0 & 0.5 & 1.9 & 0.4 & 1.6 & 0.1 \\
\hline Std Dev (s) & & & 0.18 & 0.09 & 0.10 & 0.04 & 0.93 & 0.35 & 0.008 & 0.010 & 0.017 & 0.007 & & & 4.6 & 1.0 & 5.0 & 0.5 & 1.1 & 0.1 \\
\hline Count & 1 & 1 & 3 & 3 & 6 & 6 & 5 & 5 & 8 & 8 & 8 & 8 & 1 & 1 & 6 & 6 & 6 & 6 & 4 & 4 \\
\hline Min & & & -0.05 & 0.01 & 0.03 & 0.02 & 0.80 & 0.10 & -0.020 & 0.000 & -0.030 & 0.000 & 0.001 & 0.001 & 0.5 & 0.4 & 1.3 & 0.3 & 0.9 & 0.1 \\
\hline Max & & & 0.30 & 0.20 & 0.29 & 0.12 & 2.90 & 1.00 & 0.007 & 0.030 & 0.032 & 0.020 & 0.001 & 0.001 & 13.0 & 3.0 & 14.0 & 1.6 & 3.1 & 0.3 \\
\hline$(x+2 s)$ & & & 0.46 & & 0.29 & & 3.73 & & 0.015 & & 0.035 & & 0.001 & & 13.0 & & 13.8 & & 3.9 & \\
\hline$(x+5 s)$ & & & 1.01 & & 0.59 & & 6.53 & & 0.039 & & 0.086 & & 0.001 & & 26.9 & & 28.8 & & 7.1 & \\
\hline BGUL (95\%) & & & 0.30 & & 0.29 & & 2.90 & & 0.007 & & 0.032 & & 0.001 & & 13.0 & & 14.0 & & 3.1 & \\
\hline Notes: & (1) Individu & ally higl & lighted an & yte valuc & ies have ele & ted unc & certainities & do not & meet labora & ry QA/ & $\overline{\mathrm{QC} \text { control }}$ & ecifica & ions. Data a & not ir & ded in stat & stical st & nmary. & & & \\
\hline
\end{tabular}


Appendix A. Radiochemical Analyses of River Sediments

\begin{tabular}{|c|c|c|c|c|c|c|c|c|c|c|c|c|c|c|c|c|c|c|c|c|}
\hline \multicolumn{21}{|c|}{ Table A-17. Sediment Station: Rio Grande at Bernalillo } \\
\hline \multirow{3}{*}{$\begin{array}{c}\text { Sample } \\
\text { Date }\end{array}$} & \multirow{3}{*}{$\begin{array}{c}{ }^{3} \mathrm{H} \\
(\mathrm{nCi} / \mathbf{l})\end{array}$} & \multirow{3}{*}{$\pm \mathbf{u}$} & \multirow{3}{*}{$\begin{array}{c}{ }^{90} \mathrm{Sr} \\
\text { (pCi/g) }\end{array}$} & \multirow{3}{*}{$\pm \mathbf{u}$} & \multirow{3}{*}{$\begin{array}{c}{ }^{137} \mathrm{Cs} \\
(\mathrm{pCi} / \mathrm{g})\end{array}$} & \multirow{3}{*}{$\pm \mathbf{u}$} & \multirow{3}{*}{$\begin{array}{l}\text { Total U } \\
(\mathrm{mg} / \mathrm{kg})\end{array}$} & \multirow{3}{*}{$\pm \mathbf{u}$} & \multirow{3}{*}{$\begin{array}{l}{ }^{238} \mathrm{Pu} \\
(\mathrm{pCi} / \mathrm{g})\end{array}$} & \multirow{3}{*}{$\pm \mathbf{u}$} & \multirow{3}{*}{$\begin{array}{l}{ }^{239,240} \mathrm{Pu} \\
(\mathrm{pCi} / \mathrm{g})\end{array}$} & \multirow{3}{*}{$\pm \mathbf{u}$} & \multirow{3}{*}{$\begin{array}{l}{ }^{241} \mathrm{Am} \\
(\mathrm{pCi} / \mathrm{g})\end{array}$} & \multirow{3}{*}{$\pm \mathbf{u}$} & Gross & & Gross & & Gross & \\
\hline & & & & & & & & & & & & & & & Alpha & $\pm \mathbf{u}$ & Beta & $\pm \mathbf{u}$ & Gamma & $\pm \mathbf{u}$ \\
\hline & & & & & & & & & & & & & & & $(\mathrm{pCi} / \mathrm{g})$ & & $(\mathrm{pCi} / \mathrm{g})$ & & $(\mathrm{pCi} / \mathrm{g})$ & \\
\hline 07/01/74 & & & & & 0.23 & 0.12 & & & 0.000 & 0.000 & 0.004 & 0.001 & & & & & & & & \\
\hline 05/01/75 & & & & & & & & & 0.000 & 0.002 & 0.004 & 0.002 & & & & & & & & \\
\hline $12 / 30 / 75$ & & & & & & & & & & & & & & & & & & & & \\
\hline $04 / 02 / 76$ & 1.0 & 0.3 & 0.80 & 1.00 & 0.23 & 0.02 & 1.70 & 0.40 & 0.000 & 0.001 & 0.005 & 0.001 & & & 1.1 & 0.3 & 6.1 & 0.7 & & \\
\hline 10/05/76 & & & 0.40 & 0.30 & & & & & 0.001 & 0.001 & 0.003 & 0.001 & & & 1.4 & 0.7 & 2.4 & 0.4 & & \\
\hline 03/08/77 & & & 0.05 & 0.02 & 0.18 & 0.04 & 3.40 & 0.80 & -0.004 & 0.002 & 0.013 & 0.007 & & & 14.0 & 4.0 & 5.3 & 0.7 & & \\
\hline $10 / 19 / 77$ & 1.4 & 0.3 & 0.08 & 0.09 & -0.01 & 0.06 & 2.30 & 0.50 & 0.000 & 0.001 & 0.001 & 0.001 & & & 1.5 & 0.5 & 1.5 & 0.4 & 0.8 & 0.1 \\
\hline $03 / 07 / 78$ & & & & & 0.24 & 0.03 & 5.80 & 0.80 & -0.001 & 0.002 & -0.001 & 0.002 & & & 2.4 & 0.7 & 4.9 & 0.7 & 3.2 & 0.1 \\
\hline $03 / 01 / 79$ & & & & & 0.16 & 0.05 & 3.20 & 0.30 & 0.000 & 0.001 & 0.004 & 0.005 & & & 7.3 & 1.7 & 5.8 & 0.8 & 3.3 & 0.1 \\
\hline $02 / 01 / 80$ & & & 0.11 & 0.06 & 0.18 & 0.10 & & & -0.002 & 0.002 & 0.009 & 0.003 & -0.003 & 0.003 & 11.0 & 3.0 & 10.4 & 1.2 & 4.4 & 0.1 \\
\hline 03/01/81 & & & -1.00 & 9.00 & 0.20 & 0.00 & 3.10 & 0.30 & 0.000 & 0.001 & 0.001 & 0.001 & & & 1.4 & 0.5 & 1.4 & 0.4 & 0.2 & 0.1 \\
\hline 03/01/82 & & & & & 0.39 & 0.03 & 3.10 & 0.30 & 0.001 & 0.001 & 0.005 & 0.002 & & & 16.0 & 4.0 & 16.0 & 1.7 & 4.0 & 0.1 \\
\hline $02 / 01 / 83$ & & & & & 0.22 & 0.05 & 2.80 & 0.30 & 0.000 & 0.000 & 0.005 & 0.001 & & & & & & & 4.2 & 0.6 \\
\hline $02 / 01 / 84$ & & & & & 0.25 & 0.15 & 2.70 & 0.10 & -0.001 & 0.004 & 0.004 & 0.004 & & & & & & & & \\
\hline $02 / 01 / 85$ & & & & & & & & & & & & & & & & & & & & \\
\hline $02 / 01 / 86$ & & & & & 0.15 & 0.10 & 2.90 & 0.30 & 0.002 & 0.002 & 0.013 & 0.003 & & & & & & & 3.0 & 0.4 \\
\hline 02/01/87 & & & & & 0.13 & 0.09 & 2.80 & 0.30 & 0.000 & 0.001 & 0.004 & 0.002 & -3.260 & 0.520 & & & & & -1.6 & 0.6 \\
\hline $02 / 01 / 88$ & & & & & 0.10 & 0.09 & 2.60 & 0.30 & 0.008 & 0.002 & 0.003 & 0.002 & & & & & & & 1.7 & 0.5 \\
\hline $02 / 01 / 89$ & & & & & 0.16 & 0.06 & 2.20 & 0.20 & 0.002 & 0.001 & 0.004 & 0.001 & & & & & & & 1.3 & 0.4 \\
\hline $02 / 01 / 90$ & & & & & 0.13 & 0.08 & & & 0.004 & 0.001 & 0.004 & 0.001 & & & & & & & 4.7 & 0.6 \\
\hline 02/01/91 & & & 0.20 & 0.20 & 0.10 & 0.10 & 1.60 & 0.20 & 0.002 & 0.001 & 0.004 & 0.001 & & & 3.0 & 1.0 & 2.0 & 0.0 & 0.0 & 0.0 \\
\hline $02 / 01 / 92$ & -0.2 & 0.3 & 0.10 & 0.20 & 0.10 & 0.20 & 1.80 & 0.20 & 0.001 & 0.003 & 0.002 & 0.002 & 0.131 & 0.074 & 5.0 & 1.0 & 2.0 & 0.0 & 7.0 & 1.0 \\
\hline $02 / 01 / 93$ & 0.0 & 0.3 & 0.40 & 0.30 & 0.10 & 0.00 & 1.50 & 0.10 & 0.001 & 0.030 & 0.005 & 0.020 & 0.002 & 0.030 & 3.0 & 1.0 & 2.0 & 0.0 & 0.0 & 0.0 \\
\hline $02 / 01 / 94$ & -0.1 & 0.3 & 1.20 & 0.20 & 0.04 & & 1.40 & 0.30 & 0.002 & 0.030 & 0.001 & 0.020 & 0.005 & 0.030 & 3.0 & 1.0 & 2.0 & 0.0 & 2.0 & 0.0 \\
\hline $03 / 23 / 95$ & 0.2 & 0.3 & 0.20 & 0.20 & 0.05 & 0.02 & 1.28 & 0.23 & 0.002 & 0.001 & 0.004 & 0.002 & 0.002 & 0.001 & 1.7 & 0.5 & 1.7 & 0.2 & 1.7 & 0.2 \\
\hline $05 / 09 / 96$ & 1.0 & 1.0 & 0.00 & 0.30 & 0.02 & 0.03 & 1.44 & 0.14 & & & & & 0.003 & 0.005 & 2.4 & 0.7 & 1.4 & 0.2 & 1.3 & 0.2 \\
\hline 05/09/97 & -0.3 & 0.2 & -0.57 & 0.84 & 0.01 & & 0.92 & 0.09 & 0.001 & 0.001 & 0.002 & 0.001 & 0.002 & 0.002 & 1.1 & 2.5 & 0.9 & 0.7 & 1.8 & 0.2 \\
\hline Table A-18. St & atistical Sur & Imary $f$ & or Rio Gra & ide at $B$ & ernalillo & & & & & & & & & & & & & & & \\
\hline & & & & & & & & & & & & & & & Gross & & Gross & & Gross & \\
\hline & ${ }^{3} \mathbf{H}$ & $\pm \mathbf{u}$ & ${ }^{90} \mathrm{Sr}$ & $\pm \mathbf{u}$ & ${ }^{137} \mathrm{Cs}$ & $\pm \mathbf{u}$ & Total U & $\pm \mathbf{u}$ & ${ }^{238} \mathrm{Pu}$ & $\pm \mathbf{u}$ & ${ }^{239,240} \mathbf{P u}$ & $\pm \mathbf{u}$ & ${ }^{241} \mathrm{Am}$ & $\pm \mathbf{u}$ & Alpha & $\pm \mathbf{u}$ & Beta & $\pm \mathbf{u}$ & Gamma & $\pm \mathbf{u}$ \\
\hline Statistic & $(\mathrm{nCi} / \mathrm{l})$ & & $(\mathrm{pCi} / \mathrm{g})$ & & $(\mathrm{pCi} / \mathrm{g})$ & & $(\mathrm{mg} / \mathrm{kg})$ & & $(\mathrm{pCi} / \mathrm{g})$ & & $(\mathrm{pCi} / \mathrm{g})$ & & $(\mathrm{pCi} / \mathrm{g})$ & & $(\mathrm{pCi} / \mathrm{g})$ & & $(\mathrm{pCi} / \mathrm{g})$ & & $(\mathrm{pCi} / \mathrm{g})$ & \\
\hline Mean (x) & 0.1 & 0.4 & 0.27 & 0.19 & 0.15 & 0.1 & 2.43 & 0.31 & 0.001 & 0.004 & 0.004 & 0.004 & 0.002 & 0.012 & 4.7 & 1.4 & 4.1 & 0.5 & 2.3 & 0.3 \\
\hline Median (m) & -0.1 & 0.3 & 0.16 & 0.20 & 0.15 & 0.1 & 2.45 & 0.30 & 0.001 & 0.001 & 0.004 & 0.002 & 0.002 & 0.004 & 2.7 & 1.0 & 2.0 & 0.4 & 1.8 & 0.2 \\
\hline Std Dev (s) & 0.5 & 0.3 & 0.35 & 0.10 & 0.09 & 0.1 & 1.09 & 0.20 & 0.002 & 0.008 & 0.003 & 0.005 & 0.003 & 0.014 & 4.8 & 1.2 & 4.1 & 0.5 & 2.1 & 0.3 \\
\hline Count & 6 & 6 & 10 & 10 & 23 & 21 & 20 & 20 & 24 & 24 & 24 & 24 & 6 & 6 & 16 & 16 & 16 & 16 & 19 & 19 \\
\hline Min & -0.3 & 0.2 & 0.00 & 0.02 & -0.01 & 0.0 & 0.92 & 0.09 & -0.004 & 0.000 & -0.001 & 0.001 & -0.003 & 0.001 & 1.1 & 0.3 & 0.9 & 0.0 & -1.6 & 0.0 \\
\hline Max & 1.0 & 1.0 & 1.20 & 0.30 & 0.39 & 0.2 & 5.80 & 0.80 & 0.008 & 0.030 & 0.013 & 0.020 & 0.005 & 0.030 & 16.0 & 4.0 & 16.0 & 1.7 & 7.0 & 1.0 \\
\hline$(x+2 s)$ & 1.1 & & 0.98 & & 0.33 & & 4.61 & & 0.005 & & 0.011 & & 0.007 & & 14.3 & & 12.2 & & 6.4 & \\
\hline$(x+5 s)$ & 2.5 & & 2.04 & & 0.61 & & 7.88 & & 0.012 & & 0.021 & & 0.015 & & 28.8 & & 24.4 & & 12.6 & \\
\hline BGUL (95\%) & 1.4 & & 1.20 & & 0.38 & & 5.80 & & 0.008 & & 0.013 & & 0.005 & & 16.0 & & 16.0 & & 7.0 & \\
\hline Notes: & (1) Individu & lly hig & lighted anal & evalue & es have eley & d unc & certainities & & meet labora & $\mathrm{y}$ QA/C & $\overline{\mathrm{QC} \text { control }}$ & cifica & ons. Data al & ot incl & ded in stat & tical su & hmary. & & & \\
\hline & & & & & & & & & & & & & & & & & & & & \\
\hline
\end{tabular}


Appendix A. Radiochemical Analyses of River Sediments

\begin{tabular}{|c|c|c|c|c|c|c|c|c|c|c|c|c|c|c|c|c|c|c|c|c|}
\hline \\
\hline & & & & & \multicolumn{10}{|c|}{ Table A-19. Sediment Station: Jemez River near Jemez Pueblo } & Gross & & Gross & & Gross & \\
\hline Sample & ${ }^{3} \mathbf{H}$ & $\pm \mathbf{u}$ & ${ }^{90} \mathrm{Sr}$ & $\pm \mathbf{u}$ & ${ }^{137} \mathrm{Cs}$ & $\pm \mathbf{u}$ & Total U & $\pm \mathbf{u}$ & ${ }^{238} \mathbf{P u}$ & $\pm \mathbf{u}$ & ${ }^{239,240} \mathrm{Pu}$ & $\pm \mathbf{u}$ & ${ }^{241} \mathrm{Am}$ & $\pm \mathbf{u}$ & Alpha & $\pm \mathbf{u}$ & Beta & $\pm \mathbf{u}$ & Gamma & $\pm \mathbf{u}$ \\
\hline Date & $(\mathrm{nCi} / \mathrm{l})$ & & $(\mathrm{pCi} / \mathrm{g})$ & & $(\mathrm{pCi} / \mathrm{g})$ & & $(\mathrm{mg} / \mathrm{kg})$ & & $(\mathrm{pCi} / \mathrm{g})$ & & $(\mathrm{pCi} / \mathrm{g})$ & & $(\mathrm{pCi} / \mathrm{g})$ & & $(\mathrm{pCi} / \mathrm{g})$ & & $(\mathrm{pCi} / \mathrm{g})$ & & $(\mathrm{pCi} / \mathrm{g})$ & \\
\hline $07 / 01 / 74$ & & & & & 0.34 & 0.12 & & & 0.000 & 0.001 & 0.002 & 0.001 & & & & & & & & \\
\hline $05 / 01 / 75$ & & & & & & & & & 0.002 & 0.002 & 0.001 & 0.001 & & & & & & & & \\
\hline $09 / 01 / 75$ & & & & & & & & & -0.001 & 0.001 & 0.001 & 0.001 & & & & & & & & \\
\hline $04 / 02 / 76$ & 0.3 & 0.3 & & & 0.13 & 0.02 & 2.00 & 0.40 & 0.001 & 0.001 & 0.002 & 0.001 & & & 10.0 & 2.0 & 5.0 & 0.6 & & \\
\hline $10 / 05 / 76$ & & & & & & & & & 0.000 & 0.001 & 0.008 & 0.002 & & & 9.0 & 2.0 & 6.0 & 0.7 & & \\
\hline 03/08/77 & & & 0.16 & 0.08 & 0.32 & 0.04 & 1.70 & 0.90 & 0.000 & 0.001 & 0.006 & 0.002 & & & 12.0 & 3.6 & 4.7 & 0.6 & & \\
\hline $10 / 19 / 77$ & 3.7 & 0.4 & 0.17 & 0.10 & 0.46 & 0.06 & 2.70 & 0.60 & 0.001 & 0.001 & 0.007 & 0.002 & & & 14.0 & 3.0 & 11.7 & 1.3 & 6.5 & 0.1 \\
\hline $03 / 01 / 78$ & & & & & 0.26 & 0.07 & & & 0.000 & 0.001 & 0.002 & 0.002 & & & 4.6 & 1.1 & 4.6 & 0.6 & 8.6 & 0.2 \\
\hline $03 / 01 / 79$ & & & 0.01 & 0.11 & 0.14 & 0.03 & 2.80 & 0.30 & 0.000 & 0.002 & -0.001 & 0.002 & & & 8.0 & 2.0 & 6.3 & 0.8 & 6.0 & 0.1 \\
\hline $02 / 01 / 80$ & & & & & 0.04 & 0.04 & 2.00 & 0.20 & 0.002 & 0.001 & 0.000 & 0.003 & & & 3.3 & 0.9 & 3.5 & 0.6 & 5.2 & 0.1 \\
\hline 03/01/81 & & & & & 0.82 & 0.08 & & & 0.001 & 0.001 & 0.015 & 0.003 & & & 25.0 & 6.0 & 19.0 & 2.0 & 7.1 & 0.1 \\
\hline $03 / 01 / 82$ & & & & & 0.29 & 0.08 & 3.00 & 0.30 & 0.001 & 0.001 & 0.002 & 0.001 & & & 12.0 & 3.0 & 18.0 & 1.9 & & \\
\hline $02 / 01 / 83$ & & & & & 0.16 & 0.06 & 2.30 & 0.20 & 0.001 & 0.000 & 0.003 & 0.001 & & & & & & & 3.8 & 0.1 \\
\hline 02/01/84 & & & & & 0.53 & 0.15 & 4.10 & 0.20 & 0.005 & 0.005 & 0.010 & 0.005 & & & & & & & 8.4 & 0.6 \\
\hline 03/01/85 & & & & & 0.18 & 0.08 & 0.70 & 0.10 & 0.000 & 0.001 & 0.002 & 0.001 & & & & & & & -0.2 & 0.3 \\
\hline $02 / 01 / 86$ & & & & & 0.00 & 0.05 & 1.90 & 0.20 & 0.001 & 0.001 & 0.002 & 0.001 & & & & & & & 4.1 & 0.5 \\
\hline $02 / 01 / 87$ & & & & & 0.09 & 0.08 & 1.10 & 0.20 & 0.000 & 0.002 & 0.008 & 0.001 & & & & & & & & \\
\hline $02 / 01 / 88$ & & & & & 0.17 & 0.09 & 4.40 & 0.40 & 0.001 & 0.001 & 0.001 & 0.001 & & & & & & & 6.1 & 0.5 \\
\hline $02 / 01 / 89$ & & & & & -0.08 & 0.11 & 2.90 & 0.30 & 0.002 & 0.001 & 0.005 & 0.001 & & & & & & & 2.6 & 0.5 \\
\hline $02 / 01 / 90$ & & & & & 0.64 & 0.41 & & & 0.004 & 0.001 & 0.003 & 0.002 & & & & & & & 5.1 & 0.7 \\
\hline $02 / 01 / 91$ & & & 0.10 & 0.20 & 0.00 & 0.10 & 2.40 & 0.20 & 0.001 & 0.001 & 0.002 & 0.001 & & & 4.0 & 1.0 & 3.0 & 0.0 & 0.0 & 0.0 \\
\hline $02 / 01 / 92$ & & & 0.00 & 0.20 & & & & & 0.009 & 0.003 & 0.004 & 0.002 & 0.160 & 0.084 & 14.0 & 3.0 & 3.0 & 0.0 & 11.0 & 1.0 \\
\hline $02 / 01 / 93$ & 0.1 & 0.3 & 0.10 & 0.20 & 0.20 & 0.00 & 2.10 & 0.10 & 0.008 & 0.030 & 0.005 & 0.020 & 0.003 & 0.030 & 6.0 & 1.0 & 4.0 & 1.0 & 2.0 & 0.0 \\
\hline $02 / 01 / 94$ & -0.6 & 0.3 & 0.00 & 0.40 & 0.07 & & 0.70 & 0.10 & 0.000 & 0.030 & 0.002 & 0.020 & 0.005 & 0.030 & 4.0 & 1.0 & 3.0 & 0.0 & 2.0 & 0.0 \\
\hline 03/23/95 & 0.2 & 0.3 & 0.20 & 0.20 & 0.05 & 0.03 & 1.18 & 0.22 & 0.001 & 0.001 & 0.002 & 0.001 & 0.002 & 0.001 & 1.4 & 0.5 & 1.7 & 0.2 & 3.0 & 0.3 \\
\hline 05/09/96 & 1.0 & 2.0 & 0.40 & 0.20 & 0.08 & 0.03 & 2.61 & 0.26 & & & & & 0.005 & 0.002 & 14.5 & 6.6 & 4.1 & 0.5 & 6.6 & 0.7 \\
\hline $05 / 09 / 97$ & -0.1 & 0.7 & 2.57 & 1.99 & 0.04 & 0.01 & 1.66 & 0.17 & 0.001 & 0.001 & 0.003 & 0.001 & 0.003 & 0.001 & 2.9 & 2.7 & 2.0 & 1.0 & 3.1 & 0.3 \\
\hline
\end{tabular}

Table A-20. Statistical Summary for Jemez River near Jemez Pueblo

\begin{tabular}{|c|c|c|c|c|c|c|c|c|c|c|c|c|c|c|c|c|c|c|c|c|}
\hline & & & & & & & & & & & & & & & Gross & & Gross & & Gross & \\
\hline & ${ }^{3} \mathbf{H}$ & $\pm \mathbf{u}$ & ${ }^{9} \mathrm{Sr}$ & $\pm \mathbf{u}$ & ${ }^{137} \mathrm{Cs}$ & $\pm \mathbf{u}$ & Total U & $\pm \mathbf{u}$ & ${ }^{238} \mathbf{P u}$ & $\pm \mathbf{u}$ & ${ }^{239,240} \mathrm{Pu}$ & $\pm \mathbf{u}$ & ${ }^{241} \mathbf{A m}$ & $\pm \mathbf{u}$ & Alpha & $\pm \mathbf{u}$ & Beta & $\pm \mathbf{u}$ & Gamma & $\pm \mathbf{u}$ \\
\hline Statistic & $(\mathrm{nCi} / \mathrm{l})$ & & $(\mathrm{pCi} / \mathrm{g})$ & & $(\mathrm{pCi} / \mathrm{g})$ & & $(\mathrm{mg} / \mathrm{kg})$ & & $(\mathrm{pCi} / \mathrm{g})$ & & $(\mathrm{pCi} / \mathrm{g})$ & & $(\mathrm{pCi} / \mathrm{g})$ & & $(\mathrm{pCi} / \mathrm{g})$ & & $(\mathrm{pCi} / \mathrm{g})$ & & $(\mathrm{pCi} / \mathrm{g})$ & \\
\hline Mean (x) & 0.1 & 0.7 & 0.13 & 0.19 & 0.21 & 0.08 & 2.22 & 0.28 & 0.002 & 0.004 & 0.004 & 0.003 & 0.004 & 0.013 & 9.0 & 2.5 & 6.2 & 0.7 & 4.8 & 0.3 \\
\hline Median (m) & 0.1 & 0.3 & 0.10 & 0.20 & 0.16 & 0.07 & 2.10 & 0.20 & 0.001 & 0.001 & 0.002 & 0.001 & 0.003 & 0.002 & 8.5 & 2.0 & 4.4 & 0.6 & 5.1 & 0.3 \\
\hline Std Dev (s) & 0.6 & 0.7 & 0.13 & 0.09 & 0.22 & 0.08 & 1.00 & 0.19 & 0.002 & 0.008 & 0.004 & 0.005 & 0.001 & 0.016 & 6.1 & 1.8 & 5.3 & 0.6 & 3.0 & 0.3 \\
\hline Count & 5 & 5 & 9 & 9 & 23 & 22 & 19 & 19 & 26 & 26 & 26 & 26 & 5 & 5 & 16 & 16 & 16 & 16 & 19 & 19 \\
\hline Min & -0.6 & 0.3 & 0.00 & 0.08 & -0.08 & 0.00 & 0.70 & 0.10 & -0.001 & 0.000 & -0.001 & 0.001 & 0.002 & 0.001 & 1.4 & 0.5 & 1.7 & 0.0 & -0.2 & 0.0 \\
\hline Max & 1.0 & 2.0 & 0.40 & 0.40 & 0.82 & 0.41 & 4.40 & 0.90 & 0.009 & 0.030 & 0.015 & 0.020 & 0.005 & 0.030 & 25.0 & 6.6 & 19.0 & 2.0 & 11.0 & 1.0 \\
\hline$(x+2 s)$ & 1.3 & & 0.38 & & 0.66 & & 4.22 & & 0.006 & & 0.011 & & 0.006 & & 21.3 & & 16.9 & & 10.7 & \\
\hline$(x+5 s)$ & 3.0 & & 0.76 & & 1.32 & & 7.21 & & 0.014 & & 0.021 & & 0.010 & & 39.6 & & 32.8 & & 19.6 & \\
\hline BGUL (95\%) & 3.7 & & 0.40 & & 0.81 & & 4.40 & & 0.009 & & 0.014 & & 0.005 & & 25.0 & & 19.0 & & 11.0 & \\
\hline
\end{tabular}

Notes: (1) Individually highlighted analyte values have elevated uncertainities or do not meet laboratory QA/QC control specifications. Data are not included in statistical summary. (2) Radioactive counting uncertainities are shown as $\pm \mathrm{u}\left(1\right.$ standard deviation, except for ${ }^{3} \mathrm{H}$, which is 3 standard deviations). 
Appendix A. Radiochemical Analyses of River Sediments

\begin{tabular}{|c|c|c|c|c|c|c|c|c|c|c|c|c|c|c|c|c|c|c|c|c|}
\hline \multicolumn{21}{|c|}{ Table A-21. Summary Statistics: All Regional River Sediment Stations (Combined Group I and Group II Stations) } \\
\hline & & & & & & & & & & & & & & & Gross & & Gross & & Gross & \\
\hline & ${ }^{3} \mathbf{H}$ & $\pm \mathbf{u}$ & ${ }^{90} \mathrm{Sr}$ & $\pm \mathbf{u}$ & ${ }^{137} \mathrm{Cs}$ & $\pm \mathbf{u}$ & Total U & $\pm \mathbf{u}$ & ${ }^{238} \mathrm{Pu}$ & $\pm \mathbf{u}$ & ${ }^{239,240} \mathrm{Pu}$ & $\pm \mathbf{u}$ & ${ }^{241} \mathrm{Am}$ & $\pm \mathbf{u}$ & Alpha & $\pm \mathbf{u}$ & Beta & $\pm \mathbf{u}$ & Gamma & $\pm \mathbf{u}$ \\
\hline Statistic & $(\mathrm{nCi} / \mathrm{l})$ & & $(\mathrm{pCi} / \mathrm{g})$ & & $(\mathrm{pCi} / \mathrm{g})$ & & $(\mathrm{mg} / \mathrm{kg})$ & & $(\mathrm{pCi} / \mathrm{g})$ & & $(\mathrm{pCi} / \mathrm{g})$ & & $(\mathrm{pCi} / \mathrm{g})$ & & $(\mathrm{pCi} / \mathrm{g})$ & & $(\mathrm{pCi} / \mathrm{g})$ & & $(\mathrm{pCi} / \mathrm{g})$ & \\
\hline Mean (x) & 0.1 & 0.4 & 0.22 & 0.16 & 0.15 & 0.07 & 2.26 & 0.29 & 0.001 & 0.004 & 0.004 & 0.003 & 0.005 & 0.009 & 4.2 & 1.3 & 3.8 & 0.5 & 2.5 & 0.3 \\
\hline Median (m) & 0.0 & 0.3 & 0.10 & 0.20 & 0.12 & 0.06 & 2.25 & 0.27 & 0.000 & 0.001 & 0.003 & 0.001 & 0.003 & 0.003 & 3.0 & 1.0 & 3.0 & 0.4 & 2.4 & 0.2 \\
\hline Std Dev (s) & 0.4 & 0.3 & 0.42 & 0.10 & 0.13 & 0.07 & 1.07 & 0.19 & 0.004 & 0.008 & 0.009 & 0.005 & 0.013 & 0.012 & 3.9 & 1.3 & 3.3 & 0.4 & 2.4 & 0.3 \\
\hline Count & 54 & 54 & 76 & 76 & 190 & 186 & 158 & 158 & 211 & 211 & 210 & 210 & 38 & 38 & 119 & 119 & 118 & 118 & 134 & 134 \\
\hline Min & -0.6 & 0.2 & -0.16 & 0.01 & -0.14 & 0.00 & 0.30 & 0.00 & -0.020 & 0.000 & -0.030 & 0.000 & -0.003 & 0.001 & 0.5 & 0.0 & 0.5 & 0.0 & -5.5 & 0.0 \\
\hline Max & 1.0 & 2.0 & 2.50 & 0.50 & 0.82 & 0.63 & 8.50 & 1.20 & 0.023 & 0.030 & 0.093 & 0.020 & 0.080 & 0.030 & 25.0 & 9.5 & 19.0 & 2.0 & 11.0 & 1.0 \\
\hline$(x+2 s)$ & 0.8 & & 1.06 & & 0.41 & & 4.39 & & 0.008 & & 0.022 & & 0.030 & & 11.9 & & 10.4 & & 7.2 & \\
\hline$(x+5 s)$ & 1.9 & & 2.31 & & 0.80 & & 7.59 & & 0.019 & & 0.050 & & 0.068 & & 23.5 & & 20.3 & & 14.3 & \\
\hline BGUL (95\%) & 1.0 & & 1.64 & & 0.53 & & 4.51 & & 0.009 & & 0.020 & & 0.048 & & 14.3 & & 15.1 & & 8.4 & \\
\hline \multicolumn{21}{|c|}{ Table A-22. Summary Statistics: Group I River Sediment Stations (Chamita, Embudo, Cochiti Spillway, Bernalillo, and Jemez) } \\
\hline & & & & & & & & & & & & & & & Gross & & Gross & & Gross & \\
\hline & ${ }^{3} \mathbf{H}$ & $\pm \mathbf{u}$ & ${ }^{90} \mathrm{Sr}$ & $\pm \mathbf{u}$ & ${ }^{137} \mathrm{Cs}$ & $\pm \mathbf{u}$ & Total U & $\pm \mathbf{u}$ & ${ }^{238} \mathrm{Pu}$ & $\pm \mathbf{u}$ & ${ }^{239,240} \mathrm{Pu}$ & $\pm \mathbf{u}$ & ${ }^{241} \mathrm{Am}$ & $\pm \mathbf{u}$ & Alpha & $\pm \mathbf{u}$ & Beta & $\pm \mathbf{u}$ & Gamma & $\pm \mathbf{u}$ \\
\hline Statistic & $(\mathrm{nCi} / \mathrm{l})$ & & $(\mathrm{pCi} / \mathrm{g})$ & & $(\mathrm{pCi} / \mathrm{g})$ & & $(\mathrm{mg} / \mathrm{kg})$ & & $(\mathrm{pCi} / \mathrm{g})$ & & $(\mathrm{pCi} / \mathrm{g})$ & & $(\mathrm{pCi} / \mathrm{g})$ & & $(\mathrm{pCi} / \mathrm{g})$ & & $(\mathrm{pCi} / \mathrm{g})$ & & $(\mathrm{pCi} / \mathrm{g})$ & \\
\hline Mean (x) & 0.1 & 0.5 & 0.17 & 0.16 & 0.15 & 0.07 & 2.09 & 0.30 & 0.001 & 0.004 & 0.003 & 0.003 & 0.006 & 0.012 & 4.9 & 1.5 & 4.0 & 0.5 & 2.6 & 0.3 \\
\hline Median (m) & 0.0 & 0.3 & 0.10 & 0.20 & 0.12 & 0.06 & 1.90 & 0.22 & 0.001 & 0.001 & 0.002 & 0.001 & 0.003 & 0.003 & 3.0 & 1.0 & 2.9 & 0.4 & 2.2 & 0.2 \\
\hline Std Dev (s) & 0.4 & 0.4 & 0.27 & 0.09 & 0.14 & 0.08 & 0.95 & 0.21 & 0.003 & 0.008 & 0.005 & 0.005 & 0.017 & 0.014 & 4.8 & 1.6 & 3.9 & 0.5 & 2.7 & 0.3 \\
\hline Count & 24 & 24 & 43 & 43 & 99 & 96 & 83 & 83 & 111 & 111 & 111 & 111 & 22 & 22 & 68 & 68 & 68 & 68 & 81 & 81 \\
\hline Min & -0.6 & 0.2 & -0.15 & 0.01 & -0.08 & 0.00 & 0.30 & 0.09 & -0.020 & 0.000 & -0.030 & 0.000 & -0.003 & 0.001 & 0.5 & 0.2 & 0.5 & 0.0 & -4.1 & 0.0 \\
\hline Max & 1.0 & 2.0 & 1.20 & 0.40 & 0.82 & 0.63 & 5.80 & 1.20 & 0.011 & 0.030 & 0.032 & 0.020 & 0.080 & 0.030 & 25.0 & 9.5 & 19.0 & 2.0 & 11.0 & 1.0 \\
\hline$(x+2 s)$ & 0.9 & & 0.70 & & 0.43 & & 4.00 & & 0.007 & & 0.013 & & 0.039 & & 14.5 & & 11.7 & & 8.1 & \\
\hline$(x+5 s)$ & 2.1 & & 1.50 & & 0.85 & & 6.85 & & 0.017 & & 0.029 & & 0.089 & & 28.8 & & 23.3 & & 16.3 & \\
\hline BGUL (95\%) & 1.0 & & 0.98 & & 0.56 & & 4.49 & & 0.009 & & 0.013 & & 0.076 & & 15.7 & & 17.6 & & 8.8 & \\
\hline \multicolumn{21}{|c|}{ Table A-23. Summary Statistics: Group II River Sediment Stations (Otowi, Sandia, Pajarito, Ancho, and Frijoles) } \\
\hline & & & & & & & & & & & & & & & Gross & & Gross & & Gross & \\
\hline & ${ }^{3} \mathbf{H}$ & $\pm \mathbf{u}$ & ${ }^{90} \mathrm{Sr}$ & $\pm \mathbf{u}$ & ${ }^{137} \mathrm{Cs}$ & $\pm \mathbf{u}$ & Total U & $\underline{ \pm} \mathbf{u}$ & ${ }^{238} \mathrm{Pu}$ & $\pm \mathbf{u}$ & ${ }^{239,240} \mathrm{Pu}$ & $\pm \mathbf{u}$ & ${ }^{241} \mathrm{Am}$ & $\underline{ \pm} \mathbf{u}$ & Alpha & $\pm \mathbf{u}$ & Beta & $\pm \mathbf{u}$ & Gamma & $\pm \mathbf{u}$ \\
\hline Statistic & $(\mathrm{nCi} / \mathrm{l})$ & & $(\mathrm{pCi} / \mathrm{g})$ & & $(\mathrm{pCi} / \mathrm{g})$ & & $(\mathrm{mg} / \mathrm{kg})$ & & (pCi/g) & & $(\mathrm{pCi} / \mathrm{g})$ & & $(\mathrm{pCi} / \mathrm{g})$ & & $(\mathrm{pCi} / \mathrm{g})$ & & (pCi/g) & & $(\mathrm{pCi} / \mathrm{g})$ & \\
\hline Mean (x) & 0.0 & 0.3 & 0.28 & 0.17 & 0.14 & 0.06 & 2.44 & 0.29 & 0.001 & 0.004 & 0.005 & 0.003 & 0.003 & 0.004 & 3.3 & 0.9 & 3.6 & 0.4 & 2.3 & 0.3 \\
\hline Median (m) & 0.1 & 0.3 & 0.12 & 0.20 & 0.12 & 0.06 & 2.50 & 0.30 & 0.000 & 0.001 & 0.003 & 0.001 & 0.003 & 0.001 & 3.0 & 1.0 & 3.2 & 0.4 & 2.8 & 0.1 \\
\hline Std Dev (s) & 0.3 & 0.1 & 0.56 & 0.11 & 0.12 & 0.05 & 1.16 & 0.17 & 0.004 & 0.007 & 0.012 & 0.005 & 0.002 & 0.007 & 1.7 & 0.4 & 2.3 & 0.4 & 1.7 & 0.3 \\
\hline Count & 30 & 30 & 33 & 33 & 91 & 90 & 75 & 75 & 100 & 100 & 99 & 99 & 16 & 16 & 51 & 51 & 50 & 50 & 53 & 53 \\
\hline Min & -0.6 & 0.3 & -0.16 & 0.01 & -0.14 & 0.00 & 0.30 & 0.00 & -0.012 & 0.000 & -0.013 & 0.000 & -0.003 & 0.001 & 1.0 & 0.0 & 0.5 & 0.0 & -5.5 & 0.0 \\
\hline Max & 0.7 & 1.0 & 2.50 & 0.50 & 0.71 & 0.44 & 8.50 & 0.90 & 0.023 & 0.030 & 0.093 & 0.020 & 0.008 & 0.030 & 7.8 & 2.0 & 11.1 & 1.2 & 5.2 & 1.0 \\
\hline$(x+2 s)$ & 0.7 & & 1.39 & & 0.38 & & 4.76 & & 0.009 & & 0.029 & & 0.007 & & 6.7 & & 8.3 & & 5.7 & \\
\hline$(x+5 s)$ & 1.7 & & 3.07 & & 0.75 & & 8.23 & & 0.021 & & 0.066 & & 0.015 & & 11.7 & & 15.2 & & 10.7 & \\
\hline BGUL (95\%) & 0.7 & & 2.27 & & 0.43 & & 4.71 & & 0.011 & & 0.033 & & 0.008 & & 7.3 & & 10.0 & & 5.0 & \\
\hline Notes: & $\begin{array}{l}\text { (1) Group I } \\
\text { (2) BGUL i }\end{array}$ & Kgrol & und values & high & ghted beca & they & are recomn & ded fo & or general c & parison & n studies. & & & & & & & & & \\
\hline
\end{tabular}


Appendix A. Radiochemical Analyses of River Sediments

\begin{tabular}{|c|c|c|c|c|c|c|c|c|c|c|c|c|c|c|c|c|c|c|c|c|}
\hline & & & & & & & & & & & & & & & Gross & & Gross & & Gross & \\
\hline Statistic & $\begin{array}{c}{ }^{3} \mathbf{H} \\
\text { Gp I } \\
\end{array}$ & Gp II & $\begin{array}{l}{ }^{90} \mathrm{Sr} \\
\mathrm{Gp} \mathrm{I} \\
\end{array}$ & Gp II & $\begin{array}{l}{ }^{137} \mathrm{Cs} \\
\mathrm{Gp} \mathrm{I} \\
\end{array}$ & Gp II & $\begin{array}{c}\text { Total U } \\
\text { Gp I } \\
\end{array}$ & Gp II & $\begin{array}{l}{ }^{238} \mathrm{Pu} \\
\mathrm{Gp} \mathrm{I} \\
\end{array}$ & Gp II & $\begin{array}{c}{ }^{239,240} \mathrm{Pu} \\
\mathrm{Gp} \mathrm{I} \\
\end{array}$ & Gp II & $\begin{array}{c}{ }^{241} \mathrm{Am} \\
\mathrm{Gp} \mathrm{I} \\
\end{array}$ & Gp II & $\begin{array}{c}\text { Alpha } \\
\text { Gp I } \\
\end{array}$ & Gp II & $\begin{array}{l}\text { Beta } \\
\text { Gp I } \\
\end{array}$ & Gp II & $\begin{array}{c}\text { Gamma } \\
\text { Gp I } \\
\end{array}$ & Gp II \\
\hline Mean (x) & 0.085 & $\mid 0.029$ & 0.173 & 0.279 & 0.153 & 0.140 & 2.091 & 2.441 & 0.001 & 0.001 & 0.003 & 0.005 & 0.006 & 0.003 & 4.882 & 3.270 & 3.960 & 3.632 & 2.643 & 2.325 \\
\hline Variance $\left(\mathrm{s}^{2}\right)$ & 0.169 & 0.114 & 0.071 & 0.311 & 0.020 & 0.015 & 0.907 & 1.341 & 0.000 & 0.000 & 0.000 & 0.000 & 0.000 & 0.000 & 22.967 & 2.810 & 14.937 & 5.367 & 7.412 & 2.825 \\
\hline Count & 24 & 30 & 43 & 33 & 99 & 91 & 83 & 75 & 111 & 100 & 111 & 99 & 22 & 16 & 68 & 51 & 68 & 50 & 81 & 53 \\
\hline Diff & 0.000 & & 0.000 & & 0.000 & & 0.000 & & 0.000 & & 0.000 & & 0.000 & & 0.000 & & 0.000 & & 0.000 & \\
\hline df & 52 & & 43 & & 187 & & 144 & & 188 & & 130 & & 22 & & 88 & & 112 & & 132 & \\
\hline t-stat & 0.554 & & -1.006 & & 0.676 & & -2.060 & & 0.231 & & -1.697 & & 1.009 & & 2.569 & & 0.574 & & 0.835 & \\
\hline t-table & 0.582 & & 2.017 & & 1.973 & & 1.977 & & 1.973 & & 1.978 & & 2.074 & & 1.987 & & 1.981 & & 1.978 & \\
\hline Significant & No & & No & & No & & Yes & & No & & No & & No & & Yes & & No & & No & \\
\hline
\end{tabular}

Notes: $\quad(1)^{3} \mathrm{H}$ and gross alpha means are higher for Group I, while the total uranium mean is higher for Group II river sediments.

(2) Highlighted values are statistically significant; two-tail value reported for t-table statistic.

Table A-25. Summary Statistics: Group I vs. Group II at 95\% Significance Level - Student's t-Test with Independent Samples and Equal Variance

\begin{tabular}{|c|c|c|c|c|c|c|c|c|c|c|c|c|c|c|c|c|c|c|c|c|}
\hline & & & & & & & & & & & & & & & Gross & & Gross & & Gross & \\
\hline Statistic & $\begin{array}{c}{ }^{3} \mathrm{H} \\
\mathbf{G p ~ I}\end{array}$ & Gp II & $\begin{array}{l}{ }^{90} \mathrm{Sr} \\
\mathrm{Gp} \mathrm{I}\end{array}$ & Gp II & $\begin{array}{l}{ }^{137} \mathrm{Cs} \\
\text { Gp I }\end{array}$ & Gp II & $\begin{array}{c}\text { Total U } \\
\text { Gp I }\end{array}$ & Gp II & $\begin{array}{l}{ }^{238} \mathrm{Pu} \\
\mathrm{Gp} \mathrm{I}\end{array}$ & Gp II & $\begin{array}{c}{ }^{239,240} \mathrm{Pu} \\
\mathrm{Gp} \mathrm{I}\end{array}$ & Gp II & $\begin{array}{c}{ }^{241} \mathrm{Am} \\
\mathrm{Gp} \mathrm{I}\end{array}$ & Gp II & $\begin{array}{c}\text { Alpha } \\
\text { Gp I }\end{array}$ & Gp II & $\begin{array}{l}\text { Beta } \\
\text { Gp I }\end{array}$ & Gp II & $\begin{array}{c}\text { Gamma } \\
\text { Gp I }\end{array}$ & Gp II \\
\hline Mean (x) & 0.085 & 0.029 & 0.173 & 0.279 & 0.153 & 0.140 & 2.091 & 2.441 & 0.001 & 0.001 & 0.003 & 0.005 & 0.006 & 0.003 & 4.882 & 3.270 & 3.960 & 3.632 & 2.643 & 2.325 \\
\hline Variance $\left(\mathrm{s}^{2}\right)$ & 0.169 & 0.114 & 0.071 & 0.311 & 0.020 & 0.015 & 0.907 & 1.341 & 0.000 & 0.000 & 0.000 & 0.000 & 0.000 & 0.000 & 22.967 & 2.810 & 14.937 & 5.367 & 7.412 & 2.825 \\
\hline Count & 24 & 30 & 43 & 33 & 99 & 91 & 83 & 75 & 111 & 100 & 111 & 99 & 22 & 16 & 68 & 51 & 68 & 50 & 81 & 53 \\
\hline Diff & 0.000 & & 0.000 & & 0.000 & & 0.000 & & 0.000 & & 0.000 & & 0.000 & & 0.000 & & 0.000 & & 0.000 & \\
\hline df & 44 & & 74 & & 188 & & 156 & & 209 & & 208 & & 36 & & 117 & & 116 & & 132 & \\
\hline t-stat & 0.542 & & -1.094 & & 0.672 & & -2.081 & & 0.234 & & -1.765 & & 0.863 & & 2.296 & & 0.534 & & 0.760 & \\
\hline t-table & 0.591 & & 1.993 & & 1.973 & & 1.975 & & 1.971 & & 1.977 & & 2.028 & & 1.980 & & 1.981 & & 1.978 & \\
\hline Significant & No & & No & & No & & Yes & & No & & No & & No & & Yes & & No & & No & \\
\hline
\end{tabular}

Notes: $\quad(1)^{3} \mathrm{H}$ and gross alpha means are higher for Group I, while the total uranium mean is higher for Group II river sediments.

(2) Highlighted values are statistically significant; two-tail value reported for t-table statistic.

Table A-26. Summary Statistics: Group I vs. Group II at 95\% Significance Level - Kruskal-Wallis Test with Independent Samples

\begin{tabular}{|c|c|c|c|c|c|c|c|c|c|c|c|c|c|c|c|c|c|c|c|c|}
\hline & & & & & & & & & & & & & & & Gross & & Gross & & Gross & \\
\hline Statistic & $\begin{array}{c}{ }^{3} \mathbf{H} \\
\text { Gp I }\end{array}$ & Gp II & $\begin{array}{l}{ }^{90} \mathrm{Sr} \\
\mathrm{Gp} \mathbf{I}\end{array}$ & Gp II & $\begin{array}{l}{ }^{137} \mathrm{Cs} \\
\text { Gp I }\end{array}$ & Gp II & $\begin{array}{c}\text { Total U } \\
\text { Gp I }\end{array}$ & Gp II & $\begin{array}{l}{ }^{238} \mathrm{Pu} \\
\mathrm{Gp} \mathrm{I}\end{array}$ & Gp II & $\begin{array}{c}{ }^{239,240} \mathrm{Pu} \\
\mathrm{Gp} \mathrm{I}\end{array}$ & Gp II & $\begin{array}{l}{ }^{241} \mathrm{Am} \\
\text { Gp I }\end{array}$ & Gp II & $\begin{array}{c}\text { Alpha } \\
\text { Gp I }\end{array}$ & Gp II & $\begin{array}{l}\text { Beta } \\
\text { Gp I }\end{array}$ & Gp II & $\begin{array}{c}\text { Gamma } \\
\text { Gp I }\end{array}$ & Gp II \\
\hline Mean (x) & 0.085 & 0.029 & 0.173 & \begin{tabular}{c|}
0.279 \\
\end{tabular} & 0.153 & 0.140 & 2.091 & 2.441 & 0.001 & 0.001 & 0.003 & 0.005 & 0.006 & 0.003 & 4.882 & 3.270 & 3.960 & 3.632 & 2.643 & 2.325 \\
\hline Variance $\left(s^{2}\right)$ & 0.169 & 0.114 & 0.071 & 0.311 & 0.020 & 0.015 & 0.907 & 1.341 & 0.000 & 0.000 & 0.000 & 0.000 & 0.000 & 0.000 & 22.967 & 2.810 & 14.937 & 5.367 & 7.412 & 2.825 \\
\hline Count & 24 & 30 & 43 & 33 & 99 & 91 & 83 & 75 & 111 & 100 & 111 & 99 & 22 & 16 & 68 & 51 & 68 & 50 & 81 & 53 \\
\hline Diff & 0.000 & & 0.000 & & 0.000 & & 0.000 & & 0.000 & & 0.000 & & 0.000 & & 0.000 & & 0.000 & & 0.000 & \\
\hline df & 1 & & 1 & & 1 & & 1 & & 1 & & 1 & & 1 & & 1 & & 1 & & 1 & \\
\hline KW-stat & 0.415 & & 0.900 & & 0.156 & & 6.470 & & 1.229 & & 6.121 & & 0.071 & & 0.024 & & 1.198 & & 0.226 & \\
\hline KW-table & 3.840 & & 3.840 & & 3.840 & & 3.840 & & 3.840 & & 3.840 & & 3.840 & & 3.840 & & 3.840 & & 3.840 & \\
\hline Significant & No & & No & & No & & Yes & & No & & Yes & & No & & No & & No & & No & \\
\hline
\end{tabular}

Notes: (1) Total uranium and ${ }^{239,240} \mathrm{Pu}$ means are higher for Group II than for Group I means.

(2) Highlighted values are statistically significant; two-tail value reported for KW-table statistic. 

APPENDIX B

RADIOCHEMICAL ANALYSES FOR RESERVOIR SEDIMENTS 
Appendix B. Radiochemical Analyses of Reservoir Sediments

\begin{tabular}{|c|c|c|c|c|c|c|c|c|c|c|c|c|c|c|c|c|c|c|c|c|c|}
\hline \multirow{3}{*}{$\begin{array}{c}\text { Table B-1. } \\
\text { Year }\end{array}$} & \multicolumn{7}{|c|}{ Rio Chama Drainage Basin: Abiquiu Reservoir } & \multirow{3}{*}{$\begin{array}{l}\text { Total U } \\
(\mathrm{mg} / \mathbf{k g})\end{array}$} & \multirow{3}{*}{$\pm \mathbf{u}$} & \multirow{3}{*}{$\begin{array}{c}{ }^{238} \mathrm{Pu} \\
(\mathrm{fCi} / \mathrm{g})\end{array}$} & \multirow{3}{*}{$\pm \mathbf{u}$} & \multirow{3}{*}{$\begin{array}{c}{ }^{239,240} \mathrm{Pu} \\
(\mathrm{fCi} / \mathrm{g})\end{array}$} & \multirow{3}{*}{$\pm \mathbf{u}$} & \multirow{3}{*}{$\begin{array}{l}{ }^{241} \mathrm{Am} \\
(\mathrm{pCi} / \mathrm{g})\end{array}$} & \multirow{3}{*}{$\pm \mathbf{u}$} & \multicolumn{2}{|l|}{ Gross } & \multirow{3}{*}{$\begin{array}{c}\text { Gross } \\
\text { Beta } \\
\text { (pCi } / \mathbf{g})\end{array}$} & \multicolumn{3}{|c|}{ Gross } \\
\hline & Location & ${ }^{3} \mathbf{H}$ & $\pm \mathbf{u}$ & ${ }^{90} \mathrm{Sr}$ & $\pm \mathbf{u}$ & ${ }^{137} \mathrm{Cs}$ & $\pm \mathbf{u}$ & & & & & & & & & Alpha & $\pm \mathbf{u}$ & & $\pm \mathbf{u}$ & Gamma & $\pm \mathbf{u}$ \\
\hline & & $(\mathrm{nCi} / \mathrm{l})$ & & $(\mathrm{pCi} / \mathrm{g})$ & & $(\mathrm{pCi} / \mathrm{g})$ & & & & & & & & & & $(\mathrm{pCi} / \mathrm{g})$ & & & & $(\mathrm{pCi} / \mathrm{g})$ & \\
\hline \multirow[t]{3}{*}{1982} & Upper & & & & & & & & & 0.4 & 0.3 & 8.0 & 0.6 & & & & & & & & \\
\hline & Middle & & & & & & & & & & & & & & & & & & & & \\
\hline & Lower & & & & & & & & & 0.6 & 0.1 & 11.4 & 0.6 & & & & & & & & \\
\hline 1984 & Upper & & & & & 1.00 & 0.46 & 3.60 & 0.60 & 0.7 & 0.2 & 16.3 & 1.2 & & & & & & & & \\
\hline & Middle & & & & & 0.78 & 0.32 & 3.90 & 0.40 & 0.5 & 0.2 & 11.0 & 1.2 & & & & & & & & \\
\hline & Lower & & & & & 0.89 & 0.42 & 3.10 & 0.60 & 0.9 & 0.2 & 10.7 & 0.8 & & & & & & & & \\
\hline 1985 & Upper & & & & & & & & & 0.3 & 0.2 & 7.8 & 0.8 & & & & & & & & \\
\hline & Middle & & & & & & & & & 1.2 & 0.2 & 9.4 & 0.8 & & & & & & & & \\
\hline & Lower & & & & & & & & & 0.5 & 0.2 & 9.1 & 0.8 & & & & & & & & \\
\hline 1986 & Upper & & & & & & & & & 0.2 & 0.1 & 6.7 & 0.3 & & & & & & & & \\
\hline & Middle & & & & & & & & & 0.3 & 0.1 & 6.3 & 0.2 & & & & & & & & \\
\hline & Lower & & & & & & & & & 0.4 & 0.1 & 9.5 & 0.3 & & & & & & & & \\
\hline 1987 & Upper & & & 0.00 & 0.10 & 0.10 & 0.08 & 3.30 & 0.30 & 0.1 & 0.0 & 0.2 & 0.1 & & & & & & & & \\
\hline & Middle & & & -0.03 & 0.10 & 0.21 & 0.09 & 3.80 & 0.40 & 0.2 & 0.0 & 5.0 & 0.3 & & & & & & & & \\
\hline & Lower & & & -0.10 & 0.20 & 0.35 & 0.11 & 3.60 & 0.40 & 0.2 & 0.0 & 6.0 & 0.3 & & & & & & & & \\
\hline 1988 & Upper & & & -0.21 & 0.08 & & & 2.90 & 0.30 & 0.1 & 0.2 & 4.7 & 0.2 & & & & & & & & \\
\hline & Middle & & & -0.04 & 0.09 & & & 2.90 & 0.30 & 0.4 & 0.1 & 10.0 & 0.4 & & & & & & & & \\
\hline & Lower & & & -0.18 & 0.09 & & & 3.40 & 0.40 & 0.4 & 0.0 & 7.6 & 0.3 & & & & & & & & \\
\hline 1989 & Upper & 0.30 & 0.30 & 0.25 & 0.26 & 0.34 & 0.09 & 3.30 & 0.30 & 0.7 & 0.1 & 4.1 & 0.2 & & & & & & & 2.3 & 0.4 \\
\hline & Middle & -0.10 & 0.30 & 2.10 & 0.35 & 0.24 & 0.13 & 3.40 & 0.30 & 0.3 & 0.1 & 3.6 & 0.1 & & & & & & & 1.3 & 0.4 \\
\hline & Lower & 0.50 & 0.30 & 0.18 & 0.27 & 0.19 & 0.08 & 1.80 & 0.20 & 0.2 & 0.1 & 3.3 & 0.2 & & & & & & & -1.3 & 0.4 \\
\hline 1990 & Upper & 0.00 & 0.20 & 0.10 & 0.20 & 0.23 & 0.09 & 2.40 & 0.20 & 0.1 & 0.1 & 0.8 & 0.1 & 0.002 & 0.001 & & & & & 0.7 & 0.4 \\
\hline & Middle & 0.30 & 0.20 & 0.31 & 0.20 & 0.33 & 0.10 & 2.50 & 0.30 & 0.2 & 0.1 & 3.7 & 0.2 & 0.003 & 0.001 & & & & & 0.9 & 0.4 \\
\hline & Lower & 0.20 & 0.20 & 0.49 & 0.20 & 0.19 & 0.09 & 2.90 & 0.30 & 0.1 & 0.1 & 3.4 & 0.4 & 0.004 & 0.001 & & & & & 1.2 & 0.4 \\
\hline 1991 & Upper & 0.00 & 0.20 & 0.20 & 0.20 & 0.25 & 0.09 & 1.90 & 0.20 & 0.3 & 0.1 & 5.4 & 0.4 & & & & & & & 1.8 & 0.4 \\
\hline & Middle & 0.20 & 0.30 & 0.20 & 0.10 & 0.28 & 0.14 & 2.50 & 0.20 & 0.3 & 0.1 & 6.0 & 0.3 & & & & & & & 3.2 & 0.5 \\
\hline & Lower & 0.10 & 0.30 & 0.20 & 0.20 & 0.49 & 0.12 & 2.20 & 0.20 & 0.4 & 0.4 & 10.2 & 0.4 & & & & & & & 3.0 & 0.5 \\
\hline 1992 & Upper & 0.30 & 0.30 & 0.20 & 0.20 & 0.20 & 0.10 & 2.20 & 0.20 & 0.1 & 0.0 & 1.8 & 0.1 & -0.064 & 0.088 & 10.0 & 2.0 & 4.0 & 0.0 & 1.8 & 0.4 \\
\hline & Middle & 0.60 & 0.30 & 0.10 & 0.20 & 0.10 & 0.10 & 1.60 & 0.20 & 0.1 & 0.0 & 0.2 & 0.0 & -0.038 & 0.068 & 3.0 & 1.0 & 2.0 & 0.0 & 1.0 & 0.4 \\
\hline & Lower & 0.20 & 0.30 & 0.00 & 0.20 & 0.00 & 0.10 & 2.30 & 0.20 & 0.0 & 0.0 & 0.3 & 0.0 & -0.090 & 0.076 & 5.0 & 2.0 & 4.0 & 0.0 & 2.9 & 0.5 \\
\hline 1993 & Upper & -0.20 & 0.30 & 0.50 & 0.40 & 0.38 & 0.09 & & & 0.3 & 0.1 & 8.3 & 0.6 & 0.002 & 0.003 & 6.0 & 1.0 & 5.0 & 1.0 & 3.0 & 1.0 \\
\hline & Middle & 0.00 & 0.30 & 0.40 & 0.20 & 0.35 & 0.10 & & & 0.1 & 0.1 & 2.4 & 0.3 & 0.004 & 0.003 & 4.0 & 1.0 & 3.0 & 0.0 & 0.0 & 1.0 \\
\hline & Lower & 0.10 & 0.30 & 0.50 & 0.20 & 0.20 & 0.09 & & & 0.1 & 0.1 & 4.7 & 0.4 & 0.002 & 0.003 & 13.0 & 3.0 & 3.0 & 0.0 & 3.0 & 1.0 \\
\hline 1994 & Upper & -0.20 & 0.30 & 0.10 & 0.50 & 0.07 & & 3.60 & 0.60 & 0.4 & 0.1 & 0.8 & 0.3 & 0.002 & 0.030 & 4.0 & 1.0 & 4.0 & 1.0 & 2.0 & 0.0 \\
\hline & Middle & -0.10 & 0.30 & 0.00 & 0.20 & 0.07 & & 1.90 & 0.20 & 0.0 & 0.1 & 0.2 & 0.1 & 0.000 & 0.030 & 8.0 & 3.0 & 6.0 & 1.0 & 1.0 & 0.0 \\
\hline & Lower & -0.30 & 0.30 & 0.00 & 0.20 & 0.08 & & 2.40 & 0.50 & 0.1 & 0.1 & 0.4 & 0.1 & -0.001 & 0.030 & 4.0 & 1.0 & 4.0 & 0.0 & 1.0 & 0.0 \\
\hline 1995 & Upper & 0.00 & 0.30 & 0.10 & 0.30 & 0.02 & 0.03 & 2.32 & 0.26 & 36.0 & 3.0 & 3.0 & 1.0 & 0.003 & 0.001 & 2.0 & 1.0 & 4.0 & 0.5 & 2.2 & 0.3 \\
\hline & Middle & 0.00 & 0.30 & 0.10 & 0.40 & 0.44 & 0.06 & 3.30 & 0.40 & 2.0 & 1.0 & 12.0 & 2.0 & 0.002 & 0.001 & 7.0 & 2.0 & 8.0 & 0.9 & 2.0 & 0.3 \\
\hline & Lower & -0.30 & 0.30 & 0.20 & 0.40 & 0.35 & 0.05 & 3.72 & 0.60 & 3.0 & 1.0 & 9.0 & 1.0 & 0.005 & 0.001 & 4.0 & 1.0 & 5.0 & 0.5 & 4.8 & 0.6 \\
\hline 1996 & Upper & & & 0.10 & 0.20 & 0.08 & 0.13 & 1.32 & 0.13 & 1.2 & 0.0 & 1.1 & 0.1 & 0.002 & 0.001 & 2.9 & 0.7 & 0.9 & 0.1 & 1.4 & 0.2 \\
\hline & Middle & & & 0.50 & 0.20 & 0.12 & 0.03 & 2.69 & 0.27 & 0.2 & 0.0 & 3.8 & 0.1 & 0.003 & 0.003 & 7.6 & 3.0 & 4.6 & 0.6 & 1.9 & 0.3 \\
\hline & Lower & & & 0.10 & 0.20 & 0.35 & 0.05 & 2.23 & 0.22 & 0.3 & 0.1 & 9.0 & 0.9 & 0.004 & 0.001 & 9.8 & 3.8 & 5.6 & 0.7 & 2.4 & 0.3 \\
\hline 1997 & Upper & & & & & & & & & & & & & & & & & & & & \\
\hline & Middle & -0.20 & 0.60 & -0.08 & 1.46 & 0.21 & 0.03 & 2.44 & 0.24 & 0.4 & 0.1 & 6.8 & 0.7 & 0.005 & 0.001 & 9.0 & 3.0 & 7.0 & 2.0 & 2.4 & 0.2 \\
\hline & Lower & 0.40 & 0.70 & 0.72 & 0.94 & 0.19 & 0.03 & 3.20 & 0.32 & 0.4 & 0.1 & 5.4 & 0.4 & 0.002 & 0.001 & 8.0 & 2.0 & 6.0 & 1.0 & 0.2 & 0.2 \\
\hline & & & & & & & & & & & & & & & & & & & & & \\
\hline
\end{tabular}


Appendix B. Radiochemical Analyses of Reservoir Sediments

\begin{tabular}{|c|c|c|c|c|c|c|c|c|c|c|c|c|c|c|c|c|c|c|c|c|c|}
\hline Table B-2. & Statistical S & umma & $y$ for & Abiqui & Rese & rvoir & & & & & & & & & & Gross & & Gross & & Gross & \\
\hline & Statistic & ${ }^{3} \mathbf{H}$ & $\pm \mathbf{u}$ & ${ }^{90} \mathrm{Sr}$ & $\pm \mathbf{u}$ & ${ }^{137} \mathrm{Cs}$ & $\pm \mathbf{u}$ & Total U & $\pm \mathbf{u}$ & ${ }^{238} \mathrm{Pu}$ & $\pm \mathbf{u}$ & ${ }^{239,240} \mathrm{Pu}$ & $\pm \mathbf{u}$ & ${ }^{241} \mathbf{A m}$ & $\pm \mathbf{u}$ & Alpha & $\pm \mathbf{u}$ & Beta & $\pm \mathbf{u}$ & Gamma & $\pm \mathbf{u}$ \\
\hline & & $(\mathrm{nCi} / \mathrm{l})$ & & $(\mathrm{pCi} / \mathrm{g})$ & & $(\mathrm{pCi} / \mathrm{g})$ & & $(\mathrm{mg} / \mathrm{kg})$ & & $(\mathrm{fCi} / \mathrm{g})$ & & $(\mathrm{fCi} / \mathrm{g})$ & & $(\mathrm{pCi} / \mathrm{g})$ & & (pCi/g) & & (pCi/g) & & $(\mathrm{pCi} / \mathrm{g})$ & \\
\hline & Mean (x) & 0.08 & 0.31 & 0.15 & 0.22 & 0.28 & 0.12 & 2.77 & 0.32 & 0.3 & 0.1 & 5.6 & 0.4 & 0.003 & 0.007 & 6.3 & 1.9 & 4.5 & 0.5 & 1.8 & 0.4 \\
\hline & Median (m) & 0.00 & 0.30 & 0.10 & 0.20 & 0.22 & 0.09 & 2.80 & 0.30 & 0.3 & 0.1 & 5.4 & 0.3 & 0.002 & 0.001 & 6.0 & 2.0 & 4.0 & 0.5 & 1.9 & 0.4 \\
\hline & Std Dev (s) & 0.25 & 0.11 & 0.20 & 0.10 & 0.23 & 0.10 & 0.70 & 0.14 & 0.3 & 0.1 & 3.9 & 0.3 & 0.002 & 0.011 & 3.1 & 1.0 & 1.8 & 0.6 & 1.2 & 0.3 \\
\hline & Count & 23 & 23 & 29 & 29 & 32 & 29 & 32 & 32 & 40 & 40 & 40 & 40 & 17 & 17 & 17 & 17 & 17 & 17 & 26 & 26 \\
\hline & Min & -0.30 & 0.20 & -0.21 & 0.08 & 0.00 & 0.03 & 1.32 & 0.13 & 0.0 & 0.0 & 0.2 & 0.0 & -0.001 & 0.001 & 2.0 & 0.7 & 0.9 & 0.0 & -1.3 & 0.0 \\
\hline & Max & 0.60 & 0.70 & 0.50 & 0.50 & 1.00 & 0.46 & 3.90 & 0.60 & 1.2 & 0.4 & 16.3 & 1.2 & 0.005 & 0.030 & 13.0 & 3.8 & 8.0 & 2.0 & 4.8 & 1.0 \\
\hline & $(x+2 s)$ & 0.58 & & 0.54 & & 0.75 & & 4.17 & & 0.9 & & 13.4 & & 0.006 & & 12.4 & & 8.0 & & 4.2 & \\
\hline & $(x+5 s)$ & 1.32 & & 1.13 & & 1.46 & & 6.26 & & 1.7 & & 24.9 & & 0.011 & & 21.6 & & 13.3 & & 7.9 & \\
\hline & BGUL (95\%) & 0.59 & & 1.70 & & 0.97 & & 3.87 & & 1.2 & & 13.9 & & 0.005 & & 13.0 & & 8.0 & & 4.6 & \\
\hline
\end{tabular}

Note: (1) Historically background was defined as the mean (x) plus two times standard deviation (s), or simply $(\mathrm{x}+2 \mathrm{~s})$.

(2) BGUL is the upper limit for background and equals the 0.95 quantile (two-tail).

Table B-3. Rio Chama Drainage Basin: El Vado Reservoir

\begin{tabular}{|c|c|c|c|c|c|c|c|c|c|c|c|c|c|c|c|c|c|c|c|c|c|}
\hline Table B-3 & Rio Cham & Drains & ge ва & isin: El & ado & Reservo & & & & & & & & & & Gross & & Gross & & Gross & \\
\hline Year & Location & ${ }^{3} \mathbf{H}$ & $\pm \mathbf{u}$ & ${ }^{90} \mathrm{Sr}$ & $\pm \mathbf{u}$ & ${ }^{137} \mathrm{Cs}$ & $\pm \mathbf{u}$ & Total U & $\pm \mathbf{u}$ & ${ }^{238} \mathrm{Pu}$ & $\pm \mathbf{u}$ & ${ }^{239,240} \mathbf{P u}$ & $\pm \mathbf{u}$ & ${ }^{241} \mathbf{A m}$ & $\pm \mathbf{u}$ & Alpha & $\pm \mathbf{u}$ & Beta & $\pm \mathbf{u}$ & Gamma & $\pm \mathbf{u}$ \\
\hline & & $(\mathrm{nCi} / \mathrm{l})$ & & $(\mathrm{pCi} / \mathrm{g})$ & & $(\mathrm{pCi} / \mathrm{g})$ & & $(\mathrm{mg} / \mathrm{kg})$ & & $(\mathrm{fCi} / \mathrm{g})$ & & $(\mathrm{fCi} / \mathrm{g})$ & & $(\mathrm{pCi} / \mathrm{g})$ & & $(\mathrm{pCi} / \mathrm{g})$ & & & & $(\mathrm{pCi} / \mathrm{g})$ & \\
\hline 1982 & Upper & & & & & & & & & 0.1 & 0.0 & 10.6 & 0.0 & & & & & & & & \\
\hline & Middle & & & & & & & & & 0.1 & 0.0 & 5.2 & 0.0 & & & & & & & & \\
\hline & Lower & & & & & & & & & 0.6 & 0.0 & 12.6 & 0.0 & & & & & & & & \\
\hline 1984 & Upper & & & & & 0.70 & 0.34 & 4.60 & 0.40 & 0.5 & 0.1 & 6.7 & 0.4 & & & & & & & & \\
\hline & Middle & & & & & 0.57 & 0.30 & 3.50 & 0.40 & 0.3 & 0.1 & 6.7 & 0.8 & & & & & & & & \\
\hline & Lower & & & & & 0.35 & 0.23 & 4.20 & 0.40 & 0.4 & 0.1 & 0.5 & 0.3 & & & & & & & & \\
\hline 1985 & Upper & & & & & & & & & 0.4 & 0.2 & 7.4 & 1.2 & & & & & & & & \\
\hline & Middle & & & & & & & & & 0.3 & 0.2 & 7.7 & 0.6 & & & & & & & & \\
\hline & Lower & & & & & & & & & 0.3 & 0.2 & 8.3 & 1.0 & & & & & & & & \\
\hline 1995 & Upper & 0.00 & 0.30 & 0.10 & 0.30 & 0.12 & 0.03 & 2.02 & 0.24 & 1.0 & 1.0 & 6.0 & 1.0 & 0.003 & 0.001 & 5.5 & 1.0 & 3.0 & 0.3 & 2.1 & 0.3 \\
\hline & Middle & -0.20 & 0.30 & 0.10 & 0.20 & 0.13 & 0.03 & 1.88 & 0.21 & 2.0 & 1.0 & 5.0 & 1.0 & 0.000 & 0.001 & 5.0 & 1.0 & 3.0 & 0.3 & 2.2 & 0.3 \\
\hline & Lower & -0.10 & 0.30 & 0.10 & 0.20 & 0.16 & 0.03 & 2.46 & 0.25 & 1.0 & 1.0 & 6.0 & 1.0 & 0.003 & 0.001 & 7.0 & 1.0 & 4.0 & 0.4 & 2.4 & 0.3 \\
\hline 1996 & Upper & & & 0.20 & 0.30 & 0.16 & 0.03 & 2.27 & 0.23 & 0.2 & 0.0 & 5.3 & 0.2 & 0.005 & 0.001 & 9.9 & 4.2 & 5.8 & 0.7 & 2.4 & 0.3 \\
\hline & Middle & & & 0.20 & 0.30 & 0.11 & 0.16 & 2.51 & 0.25 & 0.1 & 0.0 & 2.0 & 0.2 & 0.003 & 0.001 & 5.8 & 2.6 & 4.5 & 0.6 & 2.1 & 0.3 \\
\hline & Lower & & & 0.40 & 0.20 & 0.23 & 0.04 & 2.32 & 0.23 & 0.2 & 0.0 & 6.7 & 0.2 & 0.001 & 0.002 & 11.0 & 5.0 & 8.7 & 1.1 & 2.7 & 0.3 \\
\hline 1997 & Upper & 0.20 & 0.70 & 1.43 & 1.67 & 0.07 & 0.02 & 3.15 & 0.32 & 0.2 & 0.0 & 0.4 & 0.2 & 0.001 & 0.002 & 8.5 & 4.0 & 6.0 & 1.7 & 2.4 & 0.2 \\
\hline & Middle & -0.30 & 0.60 & 1.04 & 0.96 & 0.20 & 0.03 & 3.90 & 0.39 & 0.3 & 0.1 & 6.3 & 0.3 & 0.005 & 0.004 & 7.9 & 2.1 & 7.8 & 1.2 & 2.7 & 0.3 \\
\hline & Lower & 0.00 & 0.70 & 8.43 & 3.66 & 0.30 & 0.04 & 4.11 & 0.41 & 0.8 & 0.1 & 7.4 & 0.4 & 0.004 & 0.001 & 11.8 & 3.0 & 8.3 & 1.2 & 3.4 & 0.3 \\
\hline
\end{tabular}

Note: (1) Individually highlighted analyte values have elevated uncertainties or do not meet laboratory QA/QC specifications. Data are not included in the statistical summaries.

(2) Radioactive counting uncertainties are shown as $\pm \mathrm{u}\left(1\right.$ standard deviation, except for ${ }^{3} \mathrm{H}$, which is 3 standard deviations).

Table B-4. Statistical Summary for EI Vado Reservoir

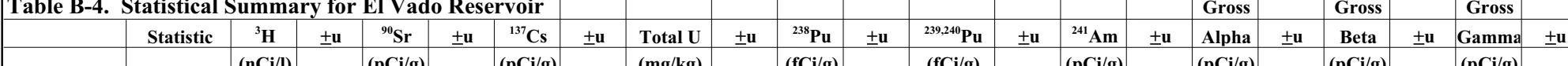

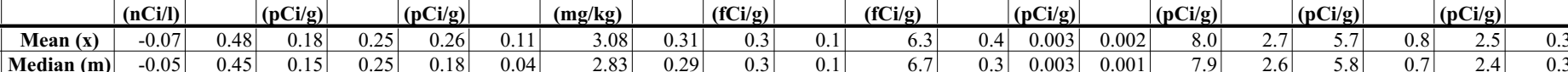
$\begin{array}{lllllllllll}\text { Median (m) } & -0.05 & 0.45 & 0.15 & 0.25 & 0.18 & 0.04 & 2.83 & 0.29 & 0.3 & 0.1\end{array}$ $\begin{array}{lrlllllllll}\text { Std Dev (s) } & 0.18 & 0.20 & 0.12 & 0.05 & 0.20 & 0.12 & 0.95 & 0.08 & 0.2 & 0.1\end{array}$

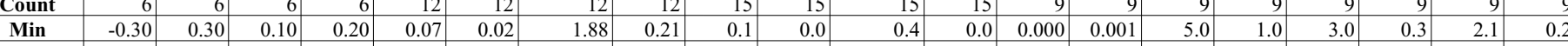

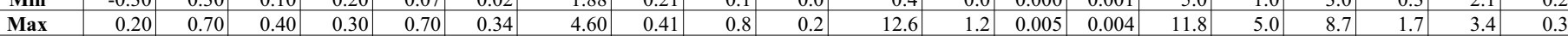

$(\mathbf{x}+\mathbf{2 s}) \quad 0.28$

\begin{tabular}{l|r|r|r|r|}
$(\mathbf{x}+\mathbf{5 s})$ & 0.81 & 0.77 & 1.23 & 7.84 \\
\hline
\end{tabular}

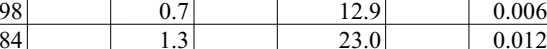

Note: (1) Historically background was defined as the mean (x) plus two times standard deviation (s), or simply ( $\mathrm{x}+2 \mathrm{~s})$ (2) BGUL is the upper limit for background and equals the 0.95 quantile (two-tail). 
Appendix B. Radiochemical Analyses of Reservoir Sediments

\begin{tabular}{|c|c|c|c|c|c|c|c|c|c|c|c|c|c|c|c|c|c|c|c|c|c|}
\hline \multicolumn{8}{|c|}{ Table B-5. Rio Chama Drainage Basin: Heron Reservoir } & \multirow{3}{*}{$\begin{array}{l}\text { Total U } \\
\text { (mg/kg) }\end{array}$} & \multirow{3}{*}{$\pm \mathbf{u}$} & \multirow{3}{*}{ 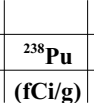 } & \multirow{3}{*}{$\pm \mathbf{u}$} & \multirow{3}{*}{$\begin{array}{c}{ }^{{ }^{239,240} \mathrm{Pu}} \\
(\mathrm{fCi} / \mathrm{g}\end{array}$} & \multirow{3}{*}{$\pm \mathbf{u}$} & \multirow{3}{*}{$\begin{array}{l}{ }^{241} \mathrm{Am} \\
(\mathrm{pCi} / \mathrm{g})\end{array}$} & \multirow{3}{*}{$\pm \mathbf{u}$} & \multicolumn{2}{|l|}{ Gross } & \multirow{3}{*}{$\begin{array}{c}\text { Gross } \\
\text { Beta } \\
(\mathrm{pCi} / \mathrm{g})\end{array}$} & \multicolumn{3}{|c|}{ Gross } \\
\hline Year & Location & ${ }^{3} \mathbf{H}$ & $\pm \mathbf{u}$ & ${ }^{90} \mathrm{Sr}$ & $\pm \mathbf{u}$ & ${ }^{137} \mathrm{Cs}$ & $\pm \mathbf{u}$ & & & & & & & & & Alpha & $\pm \mathbf{u}$ & & $\pm \mathbf{u}$ & Gamma & $\pm \mathbf{u}$ \\
\hline & & $(\mathrm{nCi} / \mathrm{l})$ & & $(\mathrm{pCi} / \mathrm{g})$ & & $(\mathrm{pCi} / \mathrm{g})$ & & & & & & & & & & $(\mathrm{pCi} / \mathrm{g})$ & & & & $(\mathrm{pCi} / \mathrm{g})$ & \\
\hline \multirow[t]{3}{*}{1982} & Upper & & & & & & & & & 0.2 & 0.0 & 7.7 & 0.0 & & & & & & & & \\
\hline & Middle & & & & & & & & & 0.7 & 0.0 & 13.5 & 0.0 & & & & & & & & \\
\hline & Lower & & & & & & & & & 0.9 & 0.0 & 19.9 & 0.0 & & & & & & & & \\
\hline \multirow[t]{3}{*}{1984} & Upper & & & & & 1.10 & 0.48 & 3.80 & 0.80 & 0.8 & 0.1 & 18.1 & 0.9 & & & & & & & & \\
\hline & Middle & & & & & 0.52 & 0.28 & 4.50 & 0.40 & 0.3 & 0.1 & 6.6 & 0.4 & & & & & & & & \\
\hline & Lower & & & & & 0.59 & 0.30 & 5.30 & 0.60 & 0.3 & 0.1 & 3.4 & 0.2 & & & & & & & & \\
\hline \multirow[t]{3}{*}{1985} & Upper & & & & & & & & & 0.7 & 0.2 & 17.4 & 1.2 & & & & & & & & \\
\hline & Middle & & & & & & & & & 0.5 & 0.2 & 11.4 & 1.6 & & & & & & & & \\
\hline & Lower & & & & & & & & & 0.2 & 0.2 & 4.7 & 0.4 & & & & & & & & \\
\hline \multirow[t]{3}{*}{1994} & Upper & -0.20 & 0.30 & 0.10 & 0.20 & 0.14 & 0.06 & 3.20 & 0.30 & 0.2 & 0.1 & 4.9 & 0.2 & 0.002 & 0.030 & 7.0 & 2.0 & 6.0 & 1.0 & 2.0 & 0.0 \\
\hline & Middle & 0.00 & 0.30 & 0.30 & 0.20 & 0.14 & 0.05 & 2.80 & 0.20 & 0.3 & 0.2 & 2.9 & 0.5 & 0.006 & 0.030 & 8.0 & 2.0 & 7.0 & 1.0 & 2.0 & 0.0 \\
\hline & Lower & 0.10 & 0.30 & 0.50 & 0.20 & 0.30 & 0.10 & 3.20 & 0.30 & 0.9 & 0.1 & 7.9 & 0.3 & 0.006 & 0.030 & 8.0 & 2.0 & 6.0 & 1.0 & 2.0 & 0.0 \\
\hline \multirow[t]{3}{*}{1995} & Upper & 0.40 & 0.30 & 0.80 & 0.30 & 0.28 & 0.05 & 3.42 & 0.44 & 3.0 & 1.0 & 12.0 & 2.0 & 0.007 & 0.001 & 10.0 & 2.0 & 5.0 & 0.5 & 3.2 & 0.4 \\
\hline & Middle & 0.50 & 0.30 & 0.30 & 0.20 & 0.29 & 0.05 & 3.46 & 0.35 & 21.0 & 2.0 & 9.0 & 1.0 & 0.003 & 0.001 & 10.0 & 2.0 & 5.0 & 0.7 & 3.3 & 0.4 \\
\hline & Lower & 0.00 & 0.30 & 0.20 & 0.20 & 0.37 & 0.06 & 3.29 & 0.33 & 19.0 & 2.0 & 11.0 & 2.0 & 0.005 & 0.001 & 12.0 & 2.0 & 5.0 & 0.5 & 3.4 & 0.4 \\
\hline \multirow[t]{3}{*}{1996} & Upper & & & 0.30 & 0.20 & 0.47 & 0.06 & 2.57 & 0.26 & 0.3 & 0.1 & 9.3 & 0.9 & 0.008 & 0.002 & 7.5 & 3.4 & 5.7 & 0.7 & 3.0 & 0.4 \\
\hline & Middle & & & 0.20 & 0.30 & 0.18 & 0.27 & 3.06 & 0.31 & 0.1 & 0.1 & 3.8 & 0.2 & 0.004 & 0.003 & 14.0 & 6.0 & 8.8 & 1.1 & 3.0 & 0.3 \\
\hline & Lower & & & 0.20 & 0.30 & 0.53 & 0.06 & 2.73 & 0.27 & 0.5 & 0.1 & 12.7 & 0.4 & 0.009 & 0.002 & 9.7 & 2.7 & 6.4 & 0.8 & 2.4 & 0.3 \\
\hline 1997 & Upper & 0.00 & 0.70 & 0.18 & 1.35 & 0.19 & 0.03 & 3.02 & 0.30 & 0.2 & 0.1 & 6.0 & 0.3 & 0.003 & 0.001 & 6.9 & 2.9 & 6.7 & 0.7 & 2.8 & 0.3 \\
\hline & Middle & -2.70 & 0.60 & 0.93 & 0.95 & 0.19 & 0.03 & 3.24 & 0.32 & 0.2 & 0.1 & 4.2 & 0.3 & 0.005 & 0.001 & 13.7 & 3.8 & 8.6 & 1.7 & 3.5 & 0.4 \\
\hline & Lower & -0.10 & 0.60 & 0.60 & 1.11 & 0.22 & 0.03 & 3.65 & 0.37 & 0.3 & 0.3 & 7.2 & 2.2 & 0.008 & 0.002 & 15.6 & 4.5 & 11.7 & 2.1 & 3.5 & 0.3 \\
\hline Note: & (1) Individua & ally highli & hted an & alyte valu & es have & e elevated & uncertai & inties or do 1 & ot meet & et laborato & $\mathrm{yQA} / \mathrm{C}$ & $2 \mathrm{C}$ specific & ions. $\mathrm{D}$ & Data are $n$ & ot incluc & Ided in the & statistic & cal summ & & & \\
\hline & (2) Radioacti & i & a unc & tainties & chow & on as $\pm \mathrm{u}$ & 1 ctond & d d di & rer & pt for ${ }^{3} \mathrm{H}$, & his is & 3 standard & leviatior & & & & & & & & \\
\hline & & & & & & & & & & & & & & & & & & & & & \\
\hline Table B- & Statistical & Summa & $y$ for & Heron I & Reserv & voir & & & & & & & & & & Gross & & Gross & & Gross & \\
\hline & Statistic & ${ }^{3} \mathrm{H}$ & $\pm \mathbf{u}$ & ${ }^{90} \mathrm{Sr}$ & $\pm \mathbf{u}$ & ${ }^{137} \mathrm{Cs}$ & $\pm \mathbf{u}$ & Total U & $\pm \mathbf{u}$ & ${ }^{238} \mathrm{Pu}$ & $\pm \mathbf{u}$ & ${ }^{239,240} \mathrm{Pu}$ & $\pm \mathbf{u}$ & ${ }^{241} \mathbf{A m}$ & $\pm \mathbf{u}$ & Alpha & $\pm \mathbf{u}$ & Beta & $\pm \mathbf{u}$ & Gamma & $\pm \mathbf{u}$ \\
\hline & & $(\mathrm{nCi} / \mathrm{l})$ & & $(\mathrm{pCi} / \mathrm{g})$ & & $(\mathrm{pCi} / \mathrm{g})$ & & $(\mathrm{mg} / \mathbf{k g})$ & & $(\mathrm{fCi} / \mathrm{g})$ & & $(\mathrm{fCi} / \mathrm{g})$ & & $(\mathrm{pCi} / \mathrm{g})$ & & $(\mathrm{pCi} / \mathrm{g})$ & & $(\mathrm{pCi} / \mathrm{g})$ & & $(\mathrm{pCi} / \mathrm{g})$ & \\
\hline & Mean (x) & 0.09 & 0.39 & 0.32 & 0.23 & 0.37 & 0.13 & 3.42 & 0.37 & 0.4 & 0.1 & 9.0 & 0.6 & 0.006 & 0.009 & 10.2 & 2.9 & 6.8 & 1.0 & 2.8 & 0.3 \\
\hline & Median (m) & 0.00 & 0.30 & 0.30 & 0.20 & 0.29 & 0.06 & 3.24 & 0.32 & 0.3 & 0.1 & 7.5 & 0.4 & 0.006 & 0.002 & 9.9 & 2.4 & 6.2 & 0.9 & 3.0 & 0.3 \\
\hline & Std Dev (s) & 0.24 & 0.16 & 0.21 & 0.05 & 0.25 & 0.14 & 0.70 & 0.15 & 0.3 & 0.1 & 5.4 & 0.6 & 0.002 & 0.013 & 3.0 & 1.3 & 2.0 & 0.5 & 0.6 & 0.2 \\
\hline & Count & 8 & 8 & 9 & 9 & 15 & 15 & 15 & 15 & 18 & 18 & 18 & 18 & 12 & 12 & 12 & 12 & 12 & 12 & 12 & 12 \\
\hline & Min & -0.20 & 0.30 & 0.10 & 0.20 & 0.14 & 0.03 & 2.57 & 0.20 & 0.1 & 0.0 & 2.9 & 0.0 & 0.002 & 0.001 & 6.9 & 2.0 & 5.0 & 0.5 & 2.0 & 0.0 \\
\hline & Max & 0.50 & 0.70 & 0.80 & 0.30 & 1.10 & 0.48 & 5.30 & 0.80 & 0.9 & 0.3 & 19.9 & 2.2 & 0.009 & 0.030 & 15.6 & 6.0 & 11.7 & 2.1 & 3.5 & 0.4 \\
\hline & $(x+2 s)$ & 0.57 & & 0.74 & & 0.87 & & 4.82 & & 0.9 & & 19.7 & & 0.010 & & 16.2 & & 10.8 & & 4.0 & \\
\hline & $(x+5 s)$ & 1.30 & & 1.38 & & 1.63 & & 6.93 & & 1.7 & & 35.7 & & 0.017 & & 25.1 & & 16.8 & & 5.8 & \\
\hline & BGUL (95\%) & 0.50 & & 0.80 & & 1.10 & & 5.30 & & 0.9 & & 19.9 & & 0.009 & & 15.6 & & 11.7 & & 3.5 & \\
\hline Note: & (1) Historica & & & & & 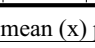 & & 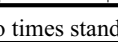 & & ation (s) & & $(x+2 s)$. & & & & & & & & & \\
\hline & (2) BGUL & e uppe & 11 & ackg1 & a a & quals $t$ & 0.9. & $\mathrm{Ie}(\mathrm{tw}$ & alit). & & & & & & & & & & & & \\
\hline
\end{tabular}


Appendix B. Radiochemical Analyses of Reservoir Sediments

\begin{tabular}{|c|c|c|c|c|c|c|c|c|c|c|c|c|c|c|c|c|c|c|c|c|c|}
\hline \multirow{3}{*}{$\begin{array}{c}\text { Table B-7 } \\
\text { Year }\end{array}$} & \multicolumn{7}{|c|}{ Rio Grande Drainage Basin: Cochiti Reservoir } & \multirow{3}{*}{$\begin{array}{l}\text { Total U } \\
(\mathrm{mg} / \mathrm{kg})\end{array}$} & \multirow{3}{*}{$\pm \mathbf{u}$} & \multirow{3}{*}{$\begin{array}{c}{ }^{238} \mathrm{Pu} \\
(\mathrm{fCi} / \mathrm{g})\end{array}$} & \multirow{3}{*}{$\pm \mathbf{u}$} & \multirow{3}{*}{$\begin{array}{r}{ }^{239,240} \mathbf{P u} \\
(\mathrm{fCi} / \mathrm{g})\end{array}$} & \multirow{3}{*}{$\pm \mathbf{u}$} & \multirow{3}{*}{$\begin{array}{c}{ }^{241} \mathrm{Am} \\
(\mathrm{pCi} / \mathrm{g})\end{array}$} & \multirow{3}{*}{$\pm \mathbf{u}$} & \multicolumn{2}{|l|}{ Gross } & \multirow{3}{*}{$\begin{array}{c}\text { Gross } \\
\text { Beta } \\
\text { (pCi/g) }\end{array}$} & & Gross & \\
\hline & Location & ${ }^{3} \mathbf{H}$ & $\pm \mathbf{u}$ & ${ }^{90} \mathrm{Sr}$ & $\pm \mathbf{u}$ & ${ }^{137} \mathrm{Cs}$ & $\pm \mathbf{u}$ & & & & & & & & & Alpha & $\pm \mathbf{u}$ & & $\pm \mathbf{u}$ & Gamma & $\pm \mathbf{u}$ \\
\hline & & $(\mathrm{nCi} / \mathrm{l})$ & & $(\mathrm{pCi} / \mathrm{g})$ & & $(\mathrm{pCi} / \mathrm{g})$ & & & & & & & & & & $(\mathrm{pCi} / \mathrm{g})$ & & & & $(\mathrm{pCi} / \mathrm{g})$ & \\
\hline 1979 & Upper & & & & & & & & & 0.2 & 0.0 & 3.9 & 0.1 & & & & & & & & \\
\hline & Middle & & & & & & & & & 1.4 & 0.2 & 30.3 & 0.7 & & & & & & & & \\
\hline & Lower & & & & & & & & & 1.4 & 0.4 & 24.4 & 1.1 & & & & & & & & \\
\hline 1982 & Upper & & & & & & & & & 0.7 & 0.1 & 15.1 & 0.8 & & & & & & & & \\
\hline & Middle & & & & & & & & & 0.8 & 0.1 & 15.6 & 1.0 & & & & & & & & \\
\hline & Lower & & & & & & & & & 0.8 & 0.1 & 17.1 & 0.8 & & & & & & & & \\
\hline 1984 & Upper & & & & & 0.58 & 0.30 & 4.10 & 0.40 & 0.1 & 0.1 & 14.7 & 0.7 & & & & & & & & \\
\hline & Middle & & & & & 0.90 & 0.30 & 4.60 & 0.40 & 1.0 & 0.1 & 16.7 & 0.8 & & & & & & & & \\
\hline & Lower & & & & & 0.81 & 0.30 & 3.60 & 0.40 & 0.9 & 0.1 & 27.7 & 1.3 & & & & & & & & \\
\hline 1985 & Upper & & & & & & & & & 2.0 & 0.2 & 29.2 & 2.4 & & & & & & & & \\
\hline & Middle & & & & & & & & & 1.2 & 0.2 & 18.9 & 1.4 & & & & & & & & \\
\hline & Lower & & & & & & & & & 1.6 & 0.6 & 24.1 & 7.3 & & & & & & & & \\
\hline 1986 & Upper & & & & & & & & & 1.0 & 0.1 & 16.5 & 0.6 & & & & & & & & \\
\hline & Middle & & & & & & & & & 1.9 & 0.2 & 30.1 & 1.6 & & & & & & & & \\
\hline & Lower & & & & & & & & & 0.9 & 0.1 & 18.2 & 0.7 & & & & & & & & \\
\hline 1987 & Upper & & & 0.07 & 0.05 & 0.26 & 0.11 & 3.80 & 0.40 & 0.0 & 0.0 & 2.6 & 0.1 & & & & & & & & \\
\hline & Middle & & & 0.03 & 0.06 & 0.15 & 0.09 & 3.80 & 0.40 & 1.1 & 0.1 & 29.7 & 1.1 & & & & & & & & \\
\hline & Lower & & & 0.08 & 0.09 & 0.51 & 0.12 & 3.80 & 0.40 & 1.4 & 0.1 & 20.2 & 0.9 & & & & & & & & \\
\hline 1988 & Upper & & & 0.05 & 0.06 & & & 2.80 & 0.30 & 0.7 & 0.1 & 12.4 & 0.5 & & & & & & & & \\
\hline & Middle & & & -0.04 & 0.06 & & & 3.70 & 0.40 & 4.1 & 0.5 & 14.8 & 1.3 & & & & & & & & \\
\hline & Lower & & & -0.07 & 0.06 & & & 3.90 & 0.40 & 0.3 & 0.1 & 9.0 & 0.6 & & & & & & & & \\
\hline 1989 & Upper & 1.00 & 0.30 & 0.11 & 0.37 & 0.43 & 0.10 & 3.20 & 0.30 & 0.7 & 0.1 & 12.9 & 0.5 & 0.041 & 0.001 & & & & & 3.5 & 0.5 \\
\hline & Middle & 0.70 & 0.30 & 0.44 & 0.39 & 0.41 & 0.12 & 4.20 & 0.40 & 5.1 & 0.4 & 133.0 & 7.0 & 0.037 & 0.003 & & & & & 4.0 & 0.5 \\
\hline & Lower & 0.40 & 0.30 & 0.44 & 0.39 & 0.60 & 0.13 & 4.20 & 0.40 & 1.7 & 0.1 & 2.0 & 0.3 & 0.009 & 0.001 & & & & & 3.5 & 0.5 \\
\hline 1990 & Upper & 0.00 & 0.30 & & & 0.29 & 0.12 & 2.90 & 0.30 & 7.0 & 0.1 & 20.9 & 1.1 & 0.020 & 0.004 & & & & & 1.6 & 0.5 \\
\hline & Middle & 0.20 & 0.30 & & & 0.55 & 0.13 & 4.60 & 0.40 & 1.1 & 0.1 & 22.5 & 1.4 & 0.016 & 0.002 & & & & & 3.1 & 0.5 \\
\hline & Lower & 0.00 & 0.30 & & & 0.40 & 0.09 & 3.80 & 0.40 & 1.6 & 0.1 & 9.4 & 0.4 & 0.011 & 0.002 & & & & & 3.0 & 0.5 \\
\hline 1991 & Upper & -0.50 & 0.50 & 0.17 & 0.16 & 0.40 & 0.11 & & & 0.3 & 0.1 & 7.2 & 0.3 & & & & & & & 1.6 & 0.4 \\
\hline & Middle & 0.30 & 0.30 & 0.19 & 0.16 & 0.25 & 0.08 & & & 0.2 & 0.1 & 4.5 & 0.2 & & & & & & & 1.4 & 0.4 \\
\hline & Lower & -0.10 & 0.30 & 0.11 & 0.14 & 0.31 & 0.11 & & & 0.1 & 0.1 & 0.5 & 0.1 & & & & & & & 5.5 & 0.7 \\
\hline 1992 & Upper & -0.40 & 0.30 & 0.20 & 0.20 & 0.10 & 0.10 & 1.20 & 0.10 & 0.1 & 0.1 & 1.2 & 0.1 & -0.069 & 0.081 & 3.0 & 1.0 & 2.0 & 0.0 & 1.2 & 0.4 \\
\hline & Middle & 0.00 & 0.30 & 0.30 & 0.20 & 0.50 & 0.10 & 4.60 & 0.50 & 5.5 & 0.4 & 37.7 & 1.1 & -0.228 & 0.088 & 16.0 & 4.0 & 7.0 & 1.0 & 4.1 & 0.5 \\
\hline & Lower & -0.30 & 0.30 & 0.00 & 0.20 & 0.10 & 0.10 & 1.70 & 0.20 & 0.2 & 0.0 & 1.4 & 0.1 & -0.204 & 0.082 & 4.0 & 1.0 & 2.0 & 0.0 & 0.9 & 0.4 \\
\hline 1993 & Upper & -0.10 & 0.30 & 1.40 & 0.20 & 0.41 & 0.14 & & & 12.0 & 1.0 & 85.0 & 4.0 & 0.014 & 0.003 & 28.0 & 6.0 & 7.0 & 1.0 & & \\
\hline & Middle & -0.20 & 0.30 & 1.30 & 0.30 & 0.34 & 0.13 & & & 0.3 & 0.1 & 4.0 & 0.4 & 0.004 & 0.003 & 8.0 & 2.0 & 3.0 & 0.0 & & \\
\hline & Lower & 0.40 & 0.30 & 1.30 & 0.20 & 0.29 & 0.12 & & & 0.1 & 0.1 & 2.4 & 0.3 & 0.003 & 0.003 & 4.0 & 1.0 & 2.0 & 0.0 & & \\
\hline 1994 & Upper & -0.10 & 0.30 & 0.10 & 0.20 & 0.21 & 0.07 & 1.70 & 0.20 & 0.1 & 0.1 & 2.7 & 0.2 & 0.002 & 0.030 & 4.0 & 1.0 & 4.0 & 1.0 & 1.0 & 0.0 \\
\hline & Middle & -0.10 & 0.30 & 0.30 & 0.20 & 0.32 & 0.10 & 3.20 & 0.40 & 0.6 & 0.1 & 12.8 & 0.4 & 0.008 & 0.030 & 11.0 & 3.0 & 9.0 & 1.0 & 2.0 & 0.0 \\
\hline & Lower & -0.20 & 0.30 & 0.20 & 0.20 & 0.28 & 0.10 & 2.90 & 0.30 & 0.5 & 0.1 & 12.3 & 0.6 & 0.005 & 0.030 & 70.0 & 20.0 & 13.0 & 1.0 & 1.0 & 0.0 \\
\hline 1995 & Upper & -0.20 & 0.40 & 0.30 & 0.30 & 0.09 & 0.03 & 2.29 & 0.27 & 15.0 & 1.9 & 6.5 & 1.2 & 0.002 & 0.001 & 9.0 & 2.0 & 2.0 & 0.3 & 2.3 & 0.3 \\
\hline & Middle & -0.10 & 0.40 & 0.40 & 0.20 & 0.20 & 0.04 & 0.09 & 0.03 & 5.2 & 1.3 & 19.6 & 2.3 & 0.007 & 0.003 & 14.0 & 2.0 & 7.0 & 0.8 & 4.0 & 0.4 \\
\hline & Lower & -0.10 & 0.40 & 0.30 & 0.30 & 0.23 & 0.04 & 2.37 & 0.24 & 2.5 & 0.9 & 11.4 & 1.8 & 0.005 & 0.003 & 10.0 & 2.0 & 6.0 & 0.7 & 3.3 & 0.4 \\
\hline 1996 & Upper & & & 1.60 & 0.60 & 0.43 & 0.04 & 2.86 & 0.29 & 0.9 & 0.1 & 16.6 & 0.5 & 0.007 & 0.002 & 8.1 & 5.1 & 8.1 & 1.0 & 3.4 & 0.4 \\
\hline & Middle & & & 2.70 & 0.70 & 0.70 & 0.06 & 3.13 & 0.31 & 1.2 & 0.1 & 23.8 & 1.0 & 0.009 & 0.002 & 16.1 & 12.9 & 11.4 & 1.5 & 4.4 & 0.5 \\
\hline & Lower & & & 1.30 & 0.50 & 0.34 & 0.03 & 2.10 & 0.21 & 0.6 & 0.1 & 13.8 & 0.9 & 0.006 & 0.001 & 8.1 & 3.0 & 5.5 & 0.6 & 3.0 & 0.4 \\
\hline 1997 & Upper & 0.10 & 0.70 & 0.90 & 0.65 & 0.23 & 0.03 & 3.56 & 0.36 & 0.5 & 0.1 & 8.2 & 0.2 & 0.004 & 0.001 & 4.0 & 3.3 & 4.9 & 2.1 & 2.6 & 0.3 \\
\hline & Middle & -0.20 & 0.70 & 2.69 & 1.95 & 0.40 & 0.04 & 5.05 & 0.51 & 0.7 & 0.1 & 13.4 & 0.4 & 0.006 & 0.002 & 15.0 & 5.7 & 14.0 & 3.3 & 3.5 & 0.4 \\
\hline & Lower & 0.10 & 0.70 & 1.84 & 2.29 & 0.55 & 0.05 & 4.43 & 0.44 & 0.9 & 0.1 & 17.9 & 0.5 & 0.014 & 0.005 & 12.0 & 5.8 & 12.0 & 3.6 & 3.7 & 0.4 \\
\hline Note: & (1) Individu & $11 \mathrm{~g}$ & ded ana & alyte valu & s have & elevate & certal & es or do & meet & orat & $2 \mathrm{~A} / \mathrm{C}$ & specifice & ns. D & Data are & & $d$ in the & atistice & & & & \\
\hline
\end{tabular}


Appendix B. Radiochemical Analyses of Reservoir Sediments

\begin{tabular}{|c|c|c|c|c|c|c|c|c|c|c|c|c|c|c|c|c|c|c|c|c|c|}
\hline \multirow{12}{*}{ Table B-8. } & \multirow{4}{*}{\multicolumn{2}{|c|}{ 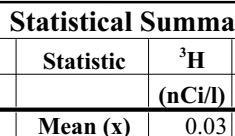 }} & $y$ for & Cochiti & Reser & voir & & \multirow{3}{*}{$\begin{array}{l}\text { Total U } \\
(\mathrm{mg} / \mathbf{k g})\end{array}$} & \multirow{3}{*}{$\pm \mathbf{u}$} & \multirow{3}{*}{$\begin{array}{c}{ }^{238} \mathrm{Pu} \\
\text { (fCi/g) }\end{array}$} & \multirow{3}{*}{$\pm \mathbf{u}$} & \multirow{3}{*}{$\begin{array}{l}{ }^{239,240} \mathrm{Pu} \\
(\mathrm{fCi} / \mathrm{g})\end{array}$} & \multirow{3}{*}{$\pm \mathbf{u}$} & \multirow{3}{*}{$\begin{array}{c}{ }^{{ }^{241}} \mathrm{Am} \\
(\mathrm{pCi} / \mathrm{g})\end{array}$} & \multirow{3}{*}{$\pm \mathbf{u}$} & \multirow{3}{*}{$\begin{array}{c}\text { Gross } \\
\text { Alpha } \\
\text { (pCi/g) }\end{array}$} & \multirow{3}{*}{$\pm \mathbf{u}$} & \multirow{3}{*}{$\begin{array}{c}\text { Gross } \\
\text { Beta } \\
\text { (pCi/g) }\end{array}$} & \multicolumn{3}{|c|}{ Gross } \\
\hline & & & $\pm \mathbf{u}$ & ${ }^{90} \mathrm{Sr}$ & $\pm \mathbf{u}$ & ${ }^{137} \mathrm{Cs}$ & $\pm \mathbf{u}$ & & & & & & & & & & & & $\pm \mathbf{u}$ & Gamma & $\pm \mathbf{u}$ \\
\hline & & & & $(\mathrm{pCi} / \mathrm{g})$ & & $(\mathrm{pCi} / \mathrm{g})$ & & & & & & & & & & & & & & $(\mathrm{pCi} / \mathrm{g})$ & \\
\hline & & & 0.37 & 0.32 & 0.20 & 0.38 & 0.11 & 3.27 & 0.34 & 1.5 & 0.2 & 19.1 & 1.1 & 0.011 & 0.006 & 13.6 & 4.5 & 6.7 & 1.1 & 2.8 & 0.4 \\
\hline & Median (m) & -0.10 & 0.30 & 0.20 & 0.20 & 0.34 & 0.10 & 3.58 & 0.40 & 0.9 & 0.1 & 15.1 & 0.7 & 0.007 & 0.003 & 9.5 & 3.0 & 6.5 & 1.0 & 3.1 & 0.4 \\
\hline & Std Dev (s) & 0.34 & 0.14 & 0.42 & 0.10 & 0.19 & 0.07 & 1.13 & 0.11 & 2.2 & 0.2 & 22.3 & 1.5 & 0.010 & 0.010 & 15.4 & 4.8 & 3.9 & 1.0 & 1.2 & 0.2 \\
\hline & Count & 24 & 24 & 24 & 24 & 33 & 33 & 30 & 30 & 45 & 45 & 45 & 45 & 21 & 21 & 18 & 18 & 18 & 18 & 24 & 24 \\
\hline & Min & -0.50 & 0.30 & -0.07 & 0.05 & 0.09 & 0.03 & 0.09 & 0.03 & 0.0 & 0.0 & 0.5 & 0.1 & 0.002 & 0.001 & 3.0 & 1.0 & 2.0 & 0.0 & 0.9 & 0.0 \\
\hline & Max & 1.00 & 0.70 & 1.40 & 0.39 & 0.90 & 0.30 & 5.05 & 0.51 & 12.0 & 1.0 & 133.0 & 7.3 & 0.041 & 0.030 & 70.0 & 20.0 & 14.0 & 3.6 & 5.5 & 0.7 \\
\hline & $(x+2 s)$ & 0.71 & & 1.15 & & 0.77 & & 5.54 & & 5.8 & & 63.6 & & 0.032 & & 44.3 & & 14.6 & & 5.3 & \\
\hline & $(x+5 s)$ & 1.73 & & 2.40 & & 1.35 & & 8.94 & & 12.3 & & 130.4 & & 0.063 & & 90.4 & & 26.4 & & 9.1 & \\
\hline & BGUL (95\%) & 0.97 & & 1.39 & & 0.87 & & 4.94 & & 8.9 & & 103.0 & & 0.041 & & 70.0 & & 14.0 & & 5.4 & \\
\hline Note: & (1) Historica & lly backg & ound w: & as defined & as the $\mathrm{r}$ & mean $(x)$ & lus two & times stan & ard devi & viation $(\mathrm{s})$ & or simp & $y(x+2 s)$ & & & & & & & & & \\
\hline & (2) BGUL is & the uppe & limit fo & or backgro & und and & equals th & $0.95 q$ & fuantile (tw & -tail). & & & & & & & & & & & & \\
\hline & & & & & & & & & & & & & & & & & & & & & \\
\hline & Rio Grand & e Drain & ge $\mathrm{Ba}$ & asin: Ri & Gran & ide Res & ryoir & & & & & & & & & & & & & & \\
\hline $\begin{array}{l}\text { Year } \\
\text { Yore }\end{array}$ & Location & ${ }^{3} \mathbf{H}$ & + & ${ }^{90} \mathrm{Sr}$ & $+u$ & ${ }^{137} \mathrm{Cs}$ & rvoir & & & & & & & & & Gross & & Gross & & Gross & \\
\hline Year & Location & $(\mathrm{nCi} / \mathrm{l})$ & $\pm \mathbf{u}$ & $\begin{array}{c}\mathrm{Sr} \\
(\mathrm{pCi} / \mathrm{g})\end{array}$ & $\pm \mathbf{u}$ & $\begin{array}{c}{ }^{C} \mathrm{Cs} \\
(\mathrm{pCi} / \mathbf{g})\end{array}$ & $\pm \mathbf{u}$ & $\begin{array}{l}\text { Total U } \\
(\mathbf{m g} / \mathbf{k g})\end{array}$ & $\pm \mathbf{u}$ & $(\mathrm{fCi} / \mathrm{g})$ & $\pm \mathbf{u}$ & $(\mathbf{f C i} / \mathbf{g})$ & $\pm \mathbf{u}$ & $\frac{{ }_{(\mathrm{nCi} / \mathbf{g})}}{\mathrm{in}}$ & $\pm \mathbf{u}$ & $\begin{array}{l}\text { Alpha } \\
\text { (pCi/g) }\end{array}$ & $\pm \mathbf{u}$ & Beta & $\pm \mathbf{u}$ & Gamma & $\pm \mathbf{u}$ \\
\hline 1982 & Upper & & & & & & & & & 0.4 & 0.3 & 8.0 & 0.6 & & & $(p(1 / g)$ & & $(p(1 / g)$ & & $(1001 / \mathrm{g})$ & \\
\hline & Middle & & & & & & & & & & & & & & & & & & & & \\
\hline & Lower & & & & & & & & & 0.6 & 0.1 & 11.4 & 0.6 & & & & & & & & \\
\hline 1986 & Upper & & & & & & & & & 0.1 & 0.1 & 4.9 & 0.4 & & & & & & & & \\
\hline & Middle & & & & & & & & & 0.5 & 0.1 & 9.5 & 0.3 & & & & & & & & \\
\hline & Lower & & & & & & & & & 2.2 & 0.2 & 38.8 & 2.7 & & & & & & & & \\
\hline 1995 & Upper & 0.20 & 0.40 & 0.70 & 0.40 & 0.12 & 0.02 & 2.85 & 0.48 & 1.0 & 1.0 & 8.0 & 1.0 & 0.004 & 0.002 & 5.0 & 1.0 & 3.0 & 0.4 & 2.9 & 0.3 \\
\hline & Middle & 0.30 & 0.40 & 0.40 & 0.50 & 0.26 & 0.04 & 2.82 & 0.28 & 1.0 & 1.0 & 11.0 & 1.0 & 0.004 & 0.001 & 7.0 & 1.0 & 5.0 & 0.5 & 2.8 & 0.3 \\
\hline & Lower & -0.10 & 0.40 & 0.40 & 0.40 & 0.23 & 0.04 & 2.84 & 0.28 & 1.0 & 1.0 & 8.0 & 1.0 & 0.001 & 0.001 & 6.0 & 1.0 & 4.0 & 0.4 & 3.3 & 0.4 \\
\hline 1996 & Upper & & & & & 0.42 & 0.04 & 3.02 & 0.30 & 0.7 & 0.1 & 16.5 & 0.5 & 0.007 & 0.002 & 7.5 & 3.1 & 5.5 & 0.6 & 3.4 & 0.4 \\
\hline & Middle & & & & & 0.33 & 0.03 & 3.08 & 0.31 & 0.6 & 0.1 & 14.4 & 0.4 & 0.007 & 0.002 & 8.5 & 1.5 & 7.1 & 0.8 & 3.3 & 0.4 \\
\hline & Lower & & & & & 0.49 & 0.04 & 2.84 & 0.28 & & & & & 0.007 & 0.002 & 16.4 & 6.1 & 8.5 & 1.0 & 2.9 & 0.3 \\
\hline 1997 & Upper & -0.50 & 0.60 & 0.72 & 1.01 & 0.53 & 0.05 & 3.62 & 0.36 & 0.7 & 0.2 & 20.4 & 3.2 & 0.011 & 0.002 & 9.9 & 2.6 & 7.0 & 1.1 & 3.3 & 0.3 \\
\hline & Middle & -0.20 & 0.70 & 0.70 & 0.71 & 0.51 & 0.06 & 3.51 & 0.35 & 0.8 & 0.1 & 19.6 & 1.0 & 0.009 & 0.002 & 6.9 & 1. & 5.3 & 1.0 & 3.2 & 0.3 \\
\hline & Lower & -0.20 & 0.60 & 0.62 & 0.92 & 0.39 & 0.04 & 3.24 & 0.32 & 0.8 & 0.1 & 17.7 & 0.9 & 0.006 & 0.002 & 7.3 & 1.8 & 5.8 & 1.0 & 3.0 & 0.3 \\
\hline Note: & (1) Individua & lly highli & hted an & halyte valu & s have & elevated & ncertai & inties or do & t meet & aborat & $\mathrm{QA} / \mathrm{Q}$ & 2C specific & ons. D & Data are $n$ & ot inclu & ded in the & statistic & cal summ & ries. & & \\
\hline & (2) Radioacti & ive counti & g unce & rtainties & show & $n$ as \pm u & standa & ard deviatio & , except & pt for ${ }^{3} \mathrm{H}$, & hich is & 3 standard & eviatiol & & & & & & & & \\
\hline Table B-10. & Statistical & Summ & ary for & r Rio G & ande & Reserv & & & & & & & & & & Gross & & Gross & & Gross & \\
\hline & Statistic & ${ }^{3} \mathbf{H}$ & $\pm \mathbf{u}$ & ${ }^{90} \mathrm{Sr}$ & $\pm \mathbf{u}$ & ${ }^{137} \mathrm{Cs}$ & $\pm \mathbf{u}$ & Total U & $\pm \mathbf{u}$ & ${ }^{238} \mathrm{Pu}$ & $\pm \mathbf{u}$ & ${ }^{239,240} \mathrm{Pu}$ & $\pm \mathbf{u}$ & ${ }^{241} \mathbf{A m}$ & $\pm \mathbf{u}$ & Alpha & $\pm \mathbf{u}$ & Beta & $\pm \mathbf{u}$ & Gamma & $\pm \mathbf{u}$ \\
\hline & & $(\mathrm{nCi} / \mathrm{l})$ & & $(\mathrm{pCi} / \mathrm{g})$ & & $(\mathrm{pCi} / \mathrm{g})$ & & $(\mathrm{mg} / \mathrm{kg})$ & & $(\mathrm{fCi} / \mathrm{g})$ & & $(\mathrm{fCi} / \mathrm{g})$ & & $(\mathrm{pCi} / \mathrm{g})$ & & $(\mathrm{pCi} / \mathrm{g})$ & & $(\mathrm{pCi} / \mathrm{g})$ & & $(\mathrm{pCi} / \mathrm{g})$ & \\
\hline & Mean (x) & -0.08 & 0.52 & 0.50 & 0.43 & 0.36 & 0.04 & 3.09 & 0.33 & 0.7 & 0.1 & 16.1 & 1.1 & 0.006 & 0.002 & 8.3 & 2.2 & 5.7 & 0.8 & 3.1 & 0.3 \\
\hline & Median (m) & -0.15 & 0.50 & 0.40 & 0.40 & 0.39 & 0.04 & 3.02 & 0.31 & 0.6 & 0.1 & 15.5 & 0.6 & 0.007 & 0.002 & 7.3 & 1.7 & 5.5 & 0.8 & 3.2 & 0.3 \\
\hline & Std Dev (s) & 0.29 & 0.13 & 0.17 & 0.06 & 0.14 & 0.01 & 0.30 & 0.06 & 0.6 & 0.1 & 9.5 & 1.0 & 0.003 & 0.000 & 3.3 & 1.6 & 1.7 & 0.3 & 0.2 & 0.1 \\
\hline & Count & 6 & 6 & 3 & 3 & 9 & 9 & 9 & 9 & 10 & 10 & 10 & 10 & 9 & 9 & 9 & 9 & 9 & 9 & 9 & 9 \\
\hline & Min & -0.50 & 0.40 & 0.40 & 0.40 & 0.12 & 0.02 & 2.82 & 0.28 & 0.1 & 0.1 & 4.9 & 0.3 & 0.001 & 0.001 & 5.0 & 1.0 & 3.0 & 0.4 & 2.8 & 0.3 \\
\hline & Max & 0.30 & 0.70 & 0.70 & 0.50 & 0.53 & 0.06 & 3.62 & 0.48 & 2.2 & 0.3 & 38.8 & 3.2 & 0.011 & 0.002 & 16.4 & 6.1 & 8.5 & 1.1 & 3.4 & 0.4 \\
\hline & $(x+2 s)$ & 0.50 & & 0.85 & & 0.65 & & 3.70 & & 1.8 & & 35.1 & & 0.012 & & 15.0 & & 9.0 & & 3.6 & \\
\hline & $(x+5 s)$ & 1.38 & & 1.37 & & 1.07 & & 4.61 & & 3.5 & & 63.5 & & 0.021 & & 25.0 & & 14.0 & & 4.2 & \\
\hline & BGUL (95\%) & 0.30 & & 0.70 & & 0.53 & & 3.62 & & 2.2 & & 38.8 & & 0.011 & & 16.4 & & 8.5 & & 3.4 & \\
\hline Note: & (1) Historical & lly backg & & as define & & mean $(x)$ & is two & nes stan & evi & on (s & & $x+2 s)$. & & & & & & & & & \\
\hline & (2) BGUL is & the uppe & limit fo & or backgro & $d$ and & lequals th & $0.95 \mathrm{~g}$ & uantile $(\mathrm{tw}$ & & & & & & & & & & & & & \\
\hline
\end{tabular}


Appendix B. Radiochemical Analyses of Reservoir Sediments

\begin{tabular}{|c|c|c|c|c|c|c|c|c|c|c|c|c|c|c|c|c|c|c|c|c|c|}
\hline \multirow[t]{4}{*}{ Table B-11. } & \multicolumn{3}{|c|}{ Summary Statistics: A } & \multicolumn{12}{|c|}{ Ill Reservoir Sediment Stations (Abiquiu, EI Vado, Heron, Cochiti, and Rio Grande) } & \multirow[b]{2}{*}{ Gross } & & \multirow[b]{2}{*}{ Gross } & \multirow{2}{*}{\multicolumn{2}{|c|}{ Gross }} & \multirow[b]{3}{*}{$\pm \mathbf{u}$} \\
\hline & & & & & & & & \multirow{3}{*}{$\begin{array}{l}\text { Total U } \\
(\mathrm{mg} / \mathbf{k g})\end{array}$} & \multirow{3}{*}{$\pm \mathbf{u}$} & \multirow{3}{*}{$\begin{array}{c}{ }^{238} \mathrm{Pu} \\
(\mathrm{fCi} / \mathrm{g})\end{array}$} & \multirow{3}{*}{$\pm \mathbf{u}$} & \multirow{3}{*}{$\begin{array}{c}{ }^{239,240} \mathbf{P u} \\
(\mathbf{f C i} / \mathbf{g})\end{array}$} & \multirow{3}{*}{$\pm \mathbf{u}$} & \multirow{3}{*}{$\begin{array}{l}\begin{array}{l}{ }^{241} \mathrm{Am} \\
(\mathrm{pCi} / \mathrm{g})\end{array} \\
\end{array}$} & \multirow{3}{*}{$\pm \mathbf{u}$} & & & & & & \\
\hline & Statistic & ${ }^{3} \mathrm{H}$ & $\pm \mathbf{u}$ & ${ }^{90} \mathrm{Sr}$ & $\pm \mathbf{u}$ & ${ }^{137} \mathrm{Cs}$ & $\pm \mathbf{u}$ & & & & & & & & & Alpha & $\pm \mathbf{u}$ & Beta & $\pm \mathbf{u}$ & Gamma & \\
\hline & & $(\mathrm{nCi} / \mathrm{l})$ & & $(\mathrm{pCi} / \mathrm{g})$ & & $(\mathrm{pCi} / \mathrm{g})$ & & & & & & & & & & $(\mathrm{pCi} / \mathrm{g})$ & & $(\mathrm{pCi} / \mathrm{g})$ & & $(\mathrm{pCi} / \mathrm{g})$ & \\
\hline & Mean (x) & 0.03 & 0.38 & 0.25 & 0.22 & 0.33 & 0.11 & 3.09 & 0.33 & 0.8 & 0.1 & 11.7 & 0.7 & 0.006 & 0.006 & 9.6 & 2.9 & 5.8 & $\overline{0.8}$ & 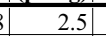 & 0.4 \\
\hline & Median (m) & 0.00 & 0.30 & 0.20 & 0.20 & 0.29 & 0.09 & 3.14 & 0.31 & 0.4 & 0.1 & 8.0 & 0.4 & 0.005 & 0.002 & 8.0 & 2.0 & 5.6 & 0.8 & 2.7 & 0.4 \\
\hline & Std Dev (s) & 0.28 & 0.15 & 0.30 & 0.10 & 0.22 & 0.10 & 0.88 & 0.12 & 1.4 & 0.1 & 15.0 & 1.0 & 0.007 & 0.010 & 8.8 & 2.9 & 2.7 & 0.7 & 1.1 & 0.2 \\
\hline & Count & 67 & 67 & 71 & 71 & 101 & 98 & 98 & 98 & 128 & 128 & 128 & 128 & 68 & 68 & 65 & 65 & 65 & 65 & 80 & 80 \\
\hline & Min & -0.50 & 0.20 & -0.21 & 0.05 & 0.00 & 0.02 & 0.09 & 0.03 & 0.0 & 0.0 & 0.2 & 0.0 & -0.001 & 0.001 & 2.0 & 0.7 & 0.9 & 0.0 & -1.3 & 0.0 \\
\hline & Max & 1.00 & 0.70 & 1.40 & 0.50 & 1.10 & 0.48 & 5.30 & 0.80 & 12.0 & 1.0 & 133.0 & 7.3 & 0.041 & 0.030 & 70.0 & 20.0 & 14.0 & 3.6 & 5.5 & 1.0 \\
\hline & $(x+2 s)$ & 0.60 & 0.68 & 0.84 & 0.43 & 0.76 & 0.30 & 4.86 & 0.57 & 3.6 & 0.4 & 41.7 & 2.7 & 0.020 & 0.025 & 27.1 & 8.7 & 11.3 & 2.2 & 4.7 & 0.7 \\
\hline & $(x+5 s)$ & 1.44 & 1.13 & 1.74 & 0.74 & 1.41 & 0.60 & 7.51 & 0.93 & 7.8 & 0.8 & 86.7 & 5.8 & 0.041 & 0.054 & 53.4 & 17.5 & 19.5 & 4.3 & 8.1 & 1.3 \\
\hline & BGUL (95\%) & 0.68 & & 1.30 & & 0.90 & & 4.62 & & 5.2 & & 38.0 & & 0.034 & & 26.6 & & 12.9 & & 4.6 & \\
\hline Notes: & (1) Historical & lly backgr & ound wa & as defined & as the 1 & mean $(\mathrm{x})$ & lus two & times stan & $\operatorname{ard} \operatorname{dev} i$ & iation $(\mathrm{s})$ & or simp & $\operatorname{ly}(x+2 s)$. & & & & & & & & & \\
\hline & (2) BGUL is & the upper & limit for & r backgro & und and & $\mathrm{d}$ equals $\mathrm{tl}$ & $0.95 \mathrm{q}$ & quantile (tw & -tail). & & & & & & & & & & & & \\
\hline & & & & & & & & & & & & & & & & & & & & & \\
\hline Table B-12. & Summary & Statisti & cs: Gr & roup I I & Reserv & oir Sed & ment & Stations & Abiqu & liu, EI & ado, I & Heron, ar & d Rio & Grande & & & & & & & \\
\hline & & & & & & & & & & & & & & & & Gross & & Gross & & Gross & \\
\hline & Statistic & ${ }^{3} \mathbf{H}$ & $\pm \mathbf{u}$ & ${ }^{90} \mathrm{Sr}$ & $\pm \mathbf{u}$ & ${ }^{137} \mathrm{Cs}$ & $\pm \mathbf{u}$ & Total U & $\pm \mathbf{u}$ & ${ }^{238} \mathrm{Pu}$ & $\pm \mathbf{u}$ & ${ }^{239,240} \mathrm{Pu}$ & $\pm \mathbf{u}$ & ${ }^{241} \mathbf{A m}$ & $\pm \mathbf{u}$ & Alpha & $\pm \mathbf{u}$ & Beta & $\pm \mathbf{u}$ & Gamma & $\pm \mathbf{u}$ \\
\hline & & $(\mathrm{nCi} / \mathrm{l})$ & & $(\mathrm{pCi} / \mathrm{g})$ & & $(\mathrm{pCi} / \mathrm{g})$ & & $(\mathrm{mg} / \mathbf{k g})$ & & $(\mathrm{fCi} / \mathrm{g})$ & & $(\mathrm{fCi} / \mathrm{g})$ & & $(\mathrm{pCi} / \mathrm{g})$ & & $(\mathrm{pCi} / \mathrm{g})$ & & $(\mathrm{pCi} / \mathrm{g})$ & & $(\mathrm{pCi} / \mathrm{g})$ & \\
\hline & Mean (x) & 0.04 & 0.38 & 0.21 & 0.24 & 0.31 & 0.11 & 3.01 & 0.33 & 0.4 & 0.1 & 7.7 & 0.5 & 0.004 & 0.005 & 8.0 & 2.4 & 5.5 & 0.8 & $\begin{array}{ll}32.3 \\
\end{array}$ & 0.3 \\
\hline & Median (m) & 0.00 & 0.30 & 0.20 & 0.20 & 0.25 & 0.06 & 3.04 & 0.30 & 0.3 & 0.1 & 6.7 & 0.3 & 0.004 & 0.002 & 7.6 & 2.0 & 5.5 & 0.7 & 2.4 & 0.3 \\
\hline & Std Dev (s) & 0.25 & 0.16 & 0.21 & 0.10 & 0.22 & 0.11 & 0.75 & 0.12 & 0.3 & 0.1 & 6.0 & 0.6 & 0.003 & 0.010 & 3.3 & 1.3 & 2.1 & 0.5 & 1.0 & 0.2 \\
\hline & Count & 43 & 43 & 47 & 47 & 68 & 65 & 68 & 68 & 83 & 83 & 83 & 83 & 47 & 47 & 47 & 47 & 47 & 47 & 56 & 56 \\
\hline & Min & -0.50 & 0.20 & -0.21 & 0.08 & 0.00 & 0.02 & 1.32 & 0.13 & 0.0 & 0.0 & 0.2 & 0.0 & -0.001 & 0.001 & 2.0 & 0.7 & 0.9 & 0.0 & -1.3 & 0.0 \\
\hline & Max & 0.60 & 0.70 & 0.80 & 0.50 & 1.10 & 0.48 & 5.30 & 0.80 & 2.2 & 0.4 & 38.8 & 3.2 & 0.011 & 0.030 & 16.4 & 6.1 & 11.7 & 2.1 & 4.8 & 1.0 \\
\hline & $(x+2 s)$ & 0.53 & 0.70 & 0.63 & 0.44 & 0.75 & 0.33 & 4.50 & 0.58 & 1.1 & 0.3 & 19.8 & 1.6 & 0.009 & 0.024 & 14.6 & 5.0 & 9.7 & 1.8 & 4.4 & 0.8 \\
\hline & $(x+5 s)$ & 1.27 & 1.17 & 1.26 & 0.74 & 1.42 & 0.66 & 6.73 & 0.95 & 2.0 & 0.5 & 37.8 & 3.3 & 0.017 & 0.053 & 24.4 & 9.1 & 15.8 & 3.3 & 7.6 & 1.4 \\
\hline & BGUL (95\%) & 0.54 & & 0.73 & & 0.98 & & 4.58 & & 1.2 & & 20.1 & & 0.010 & & 15.9 & & 9.7 & & 3.6 & \\
\hline Notes: & (1) Historical & lly backgr & $\overline{\text { ound wa }}$ & as defined & as the 1 & mean (x) & lus two & times stan & ard devi & iation (s) & $\overline{\text { or } \operatorname{simp}}$ & ly $(x+2 s)$ & & & & & & & & & \\
\hline & (2) BGUL is & the upper & limit for & r backgro & und and & $\mathrm{d}$ equals th & $0.95 q$ & fuantile (tw & -tail). & & & & & & & & & & & & \\
\hline & & & & & & & & & & & & & & & & & & & & & \\
\hline Table B-13. & Summary & Statisti & cs: Gr & roup II & Reser & voir Sec & iment & Stations & (Coch & iiti) & & & & & & & & & & & \\
\hline & & & & & & & & & & & & & & & & Gross & & Gross & & Gross & \\
\hline & Statistic & ${ }^{3} \mathbf{H}$ & $\pm \mathbf{u}$ & ${ }^{90} \mathrm{Sr}$ & $\pm \mathbf{u}$ & ${ }^{137} \mathrm{Cs}$ & $\pm \mathbf{u}$ & Total U & $\pm \mathbf{u}$ & ${ }^{238} \mathrm{Pu}$ & $\pm \mathbf{u}$ & ${ }^{239,240} \mathrm{Pu}$ & $\pm \mathbf{u}$ & ${ }^{241} \mathrm{Am}$ & $\pm \mathbf{u}$ & Alpha & $\pm \mathbf{u}$ & Beta & $\pm \mathbf{u}$ & Gamma & $\pm \mathbf{u}$ \\
\hline & & $(\mathrm{nCi} / \mathrm{l})$ & & $(\mathrm{pCi} / \mathrm{g})$ & & $(\mathrm{pCi} / \mathrm{g})$ & & $(\mathrm{mg} / \mathrm{kg})$ & & $(\mathrm{fCi} / \mathrm{g})$ & & $(\mathrm{fCi} / \mathrm{g})$ & & $(\mathrm{pCi} / \mathrm{g})$ & & $(\mathrm{pCi} / \mathrm{g})$ & & $(\mathrm{pCi} / \mathrm{g})$ & & $(\mathrm{pCi} / \mathrm{g})$ & \\
\hline & Mean (x) & 0.03 & 0.37 & 0.32 & 0.20 & 0.38 & 0.11 & 3.27 & 0.34 & 1.5 & 0.2 & 19.1 & 1.1 & 0.011 & 0.006 & 13.6 & 4.5 & 6.7 & 1.1 & 2.8 & 0.4 \\
\hline & Median (m) & -0.10 & 0.30 & 0.20 & 0.20 & 0.34 & 0.10 & 3.58 & 0.40 & 0.9 & 0.1 & 15.1 & 0.7 & 0.007 & 0.003 & 9.5 & 3.0 & 6.5 & 1.0 & 3.1 & 0.4 \\
\hline & Std Dev (s) & 0.34 & 0.14 & 0.42 & 0.10 & 0.19 & 0.07 & 1.13 & 0.11 & 2.2 & 0.2 & 22.3 & 1.5 & 0.010 & 0.010 & 15.4 & 4.8 & 3.9 & 1.0 & 1.2 & 0.2 \\
\hline & Count & 24 & 24 & 24 & 24 & 33 & 33 & 30 & 30 & 45 & 45 & 45 & 45 & 21 & 21 & 18 & 18 & 18 & 18 & 24 & 24 \\
\hline & Min & -0.50 & 0.30 & -0.07 & 0.05 & 0.09 & 0.03 & 0.09 & 0.03 & 0.0 & 0.0 & 0.5 & 0.1 & 0.002 & 0.001 & 3.0 & 1.0 & 2.0 & 0.0 & 0.9 & 0.0 \\
\hline & Max & 1.00 & 0.70 & 1.40 & 0.39 & 0.90 & 0.30 & 5.05 & 0.51 & 12.0 & 1.0 & 133.0 & 7.3 & 0.041 & 0.030 & 70.0 & 20.0 & 14.0 & 3.6 & 5.5 & 0.7 \\
\hline & $(x+2 s)$ & 0.71 & 0.64 & 1.15 & 0.40 & 0.77 & 0.25 & 5.54 & 0.55 & 5.8 & 0.5 & 63.6 & 4.0 & 0.032 & 0.026 & 44.3 & 14.2 & 14.6 & 3.1 & 5.3 & 0.7 \\
\hline & $(x+5 s)$ & 1.73 & 1.05 & 2.40 & 0.72 & 1.35 & 0.46 & 8.94 & 0.88 & 12.3 & 1.0 & 130.4 & 8.5 & 0.063 & 0.056 & 90.4 & 28.6 & 26.4 & 6.2 & 9.1 & 1.2 \\
\hline & BGUL (95\%) & 0.97 & & 1.39 & & 0.87 & & 4.94 & & 8.9 & & 103.0 & & 0.041 & & 70.0 & & 14.0 & & 5.4 & \\
\hline otes: & $\begin{array}{l}\text { (1) Historical } \\
\text { (2) BGUL is }\end{array}$ & $\begin{array}{l}\text { lly backgr } \\
\text { the upper }\end{array}$ & it for & $\begin{array}{l}\text { as definec } \\
\text { or backgro }\end{array}$ & 5 & nean $(\mathrm{x})$ & 95 & 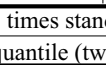 & & & & $(x+2 s)$. & & & & & & & & & \\
\hline
\end{tabular}


Appendix B. Radiochemical Analyses of Reservoir Sediments

\begin{tabular}{|c|c|c|c|c|c|c|c|c|c|c|c|c|c|c|c|c|c|c|c|c|}
\hline \multirow[b]{3}{*}{ Statistic } & \multirow{3}{*}{\begin{tabular}{|c|} 
Summary \\
${ }^{3} \mathbf{H}$ \\
Gp I \\
\end{tabular}} & \multirow{3}{*}{ Gp II } & \multirow{3}{*}{${ }^{90} \mathrm{Sr}$} & \multirow[b]{3}{*}{ Gp II } & \multirow{3}{*}{$\begin{array}{l}{ }^{137} \mathrm{Cs} \\
\mathrm{Gp} \mathrm{I}\end{array}$} & \multirow{2}{*}{\multicolumn{2}{|c|}{ Total U }} & \multirow[b]{3}{*}{ Gp II } & \multirow{3}{*}{$\begin{array}{l}{ }^{238} \mathrm{Pu} \\
\mathrm{Gp} \mathrm{I} \\
\end{array}$} & \multirow{2}{*}{\multicolumn{2}{|c|}{${ }^{239,240} \mathbf{P u}$}} & \multirow[b]{3}{*}{ Gp II } & \multirow{3}{*}{\begin{tabular}{|}
${ }^{241} \mathrm{Am}$ \\
$\mathbf{G p ~ I}$ \\
\end{tabular}} & \multirow[b]{3}{*}{ Gp II } & \multirow{3}{*}{$\begin{array}{c}\text { Gross } \\
\text { Alpha } \\
\text { Gp I } \\
\end{array}$} & \multirow[b]{3}{*}{ Gp II } & \multirow{3}{*}{$\begin{array}{c}\text { Gross } \\
\text { Beta } \\
\text { Gp I } \\
\end{array}$} & \multirow{2}{*}{\multicolumn{3}{|c|}{$\begin{array}{l}\text { Gross } \\
\text { Gamma }\end{array}$}} \\
\hline & & & & & & & & & & & & & & & & & & & & \\
\hline & & & & & & Gp II & Gp I & & & Gp II & Gp I & & & & & & & Gp II & Gp I & Gp II \\
\hline Mean (x) & 0.037 & 0.025 & 0.208 & 0.320 & 0.308 & 0.381 & 3.009 & 3.273 & 0.404 & 1.457 & 7.735 & 19.051 & 0.004 & 0.011 & 8.012 & 13.572 & 5.538 & 6.661 & 2.334 & 2.817 \\
\hline Variance $\left(s^{2}\right)$ & 0.061 & 0.116 & 0.044 & 0.173 & 0.049 & 0.038 & 0.555 & 1.286 & 0.108 & 4.718 & 36.256 & 496.130 & 0.000 & 0.000 & 10.713 & 236.40 & 4.228 & 15.598 & 1.100 & 1.561 \\
\hline Count & 43 & 24 & 47 & 24 & 68 & 33 & 68 & 30 & 83 & 45 & 83 & 45 & 47 & 21 & 47 & 18 & 47 & 18 & 56 & 24 \\
\hline Diff & 0.000 & & 0.000 & & 0.000 & & 0.000 & & 0.000 & & 0.000 & & 0.000 & & 0.000 & & 0.000 & & 0.000 & \\
\hline df & 37 & & 29 & & 71 & & 40 & & 45 & & 48 & & 21 & & 18 & & 21 & & 38 & \\
\hline t-stat & 0.154 & & -1.240 & & -1.676 & & -1.168 & & -3.232 & & -3.342 & & -2.964 & & -1.521 & & -1.148 & & -1.659 & \\
\hline t-table & 2.026 & & 2.045 & & 1.994 & & 2.021 & & 2.014 & & 2.011 & & 2.080 & & 2.101 & & 2.080 & & 2.024 & \\
\hline Significant & No & & No & & No & & No & & Yes & & Yes & & Yes & & No & & No & & No & \\
\hline
\end{tabular}

Notes: $\quad(1)^{238} \mathrm{Pu},{ }^{23,240} \mathrm{Pu}$, and ${ }^{241} \mathrm{Am}$ means are higher for Group II than for Group I reservoir sediments.

(2) Highlighted values are statistically significant; two-tail value reported for t-table statistic.

Table B-15. Summary Statistics: Group I vs. Group II at 95\% Significance Level - Student's t-Test with Independent Samples and Equal Variance

\begin{tabular}{|c|c|c|c|c|c|c|c|c|c|c|c|c|c|c|c|c|c|c|c|c|}
\hline \multirow[b]{3}{*}{ Statistic } & \multirow{3}{*}{$\begin{array}{c}{ }^{3} \mathbf{H} \\
\mathbf{G p} \mathbf{I}\end{array}$} & \multirow[b]{3}{*}{ Gp II } & \multirow{3}{*}{$\begin{array}{l}{ }^{90} \mathrm{Sr} \\
\mathrm{Gp} \mathbf{I}\end{array}$} & \multirow[b]{3}{*}{ Gp II } & \multirow{3}{*}{$\begin{array}{l}{ }^{137} \mathrm{Cs} \\
\text { Gp I } \\
\end{array}$} & \multirow{2}{*}{\multicolumn{3}{|c|}{ Total U }} & \multirow{3}{*}{$\begin{array}{l}{ }^{238} \mathrm{Pu} \\
\mathrm{Gp} \mathbf{I}\end{array}$} & \multirow{2}{*}{\multicolumn{3}{|c|}{${ }^{239,240} \mathrm{Pu}$}} & \multirow{3}{*}{$\begin{array}{r}{ }^{241} \mathrm{Am} \\
\mathbf{G p} \mathbf{I} \\
\end{array}$} & \multirow[b]{3}{*}{ Gp II } & \multirow{3}{*}{$\begin{array}{c}\text { Gross } \\
\text { Alpha } \\
\text { Gp I } \\
\end{array}$} & \multirow[b]{3}{*}{ Gp II } & \multirow{3}{*}{$\begin{array}{c}\text { Gross } \\
\text { Beta } \\
\text { Gp I } \\
\end{array}$} & \multirow{2}{*}{\multicolumn{3}{|c|}{$\begin{array}{c}\text { Gross } \\
\text { Gamma }\end{array}$}} \\
\hline & & & & & & & & & & & & & & & & & & & & \\
\hline & & & & & & Gp II & Gp I & Gp II & & Gp II & Gp I & Gp II & & & & & & Gp II & Gp I & Gp II \\
\hline Mean (x) & 0.037 & 0.025 & 0.208 & 0.320 & 0.308 & 0.381 & 3.009 & 3.273 & 0.404 & 1.457 & 7.735 & 19.051 & 0.004 & 0.011 & 8.012 & 13.572 & 5.538 & 6.661 & 2.334 & 2.817 \\
\hline Variance $\left(\mathrm{s}^{2}\right)$ & 0.061 & 0.116 & 0.044 & 0.173 & 0.049 & 0.038 & 0.555 & 1.286 & 0.108 & 4.718 & 36.256 & 496.130 & 0.000 & 0.000 & 10.713 & 236.40 & 4.228 & 15.598 & 1.100 & 1.561 \\
\hline Count & 43 & 24 & 47 & 24 & 68 & 33 & 68 & 30 & 83 & 45 & 83 & 45 & 47 & 21 & 47 & 18 & 47 & 18 & 56 & 24 \\
\hline Diff & 0.000 & & 0.000 & & 0.000 & & 0.000 & & 0.000 & & 0.000 & & 0.000 & & 0.000 & & 0.000 & & 0.000 & \\
\hline df & 65 & & 69 & & 99 & & 96 & & 126 & & 126 & & 66 & & 63 & & 63 & & 78 & \\
\hline t-stat & 0.169 & & -1.512 & & -1.602 & & -1.367 & & -4.339 & & -4.357 & & -4.247 & & -2.370 & & -1.500 & & -1.780 & \\
\hline t-table & 1.997 & & 1.995 & & 1.984 & & 1.985 & & 1.979 & & 1.979 & & 1.997 & & 1.998 & & 1.998 & & 1.991 & \\
\hline Significant & No & & No & & No & & No & & Yes & & Yes & & Yes & & Yes & & No & & No & \\
\hline
\end{tabular}

\begin{tabular}{ccccccc} 
Significant & No & No & No & No & Yes & Yes \\
\hline Notes: & $(1)^{238} \mathrm{Pu},{ }^{239,240} \mathrm{Pu},{ }^{241} \mathrm{Am}$, and Gross Alpha means are higher for Group II than for Group I reservoir sediments.
\end{tabular}

(1) ${ }^{25} \mathrm{Pu},{ }^{23,24} \mathrm{Pu},{ }^{24} \mathrm{Am}$, and Gross Alpha means are higher for Group II than for Group I reservo
(2) Highlighted values are statistically significant; two-tail value reported for t-table statistic.

Table B-16. Summary Statistics: Group I vs. Group II at 95\% Significance Level - Kruskal-Wallis Test with Independent Samples

\begin{tabular}{|c|c|c|c|c|c|c|c|c|c|c|c|c|c|c|c|c|c|c|c|c|}
\hline \multirow[b]{3}{*}{ Statistic } & \multirow{3}{*}{$\begin{array}{c}{ }^{3} \mathbf{H} \\
\text { Gp I } \\
\end{array}$} & \multirow[b]{3}{*}{ Gp II } & \multirow{3}{*}{$\begin{array}{l}{ }^{90} \mathrm{Sr} \\
\mathrm{Gp} \mathbf{I}\end{array}$} & \multirow[b]{3}{*}{ Gp II } & \multirow{3}{*}{$\begin{array}{l}{ }^{137} \mathrm{Cs} \\
\mathrm{Gp} \mathrm{I} \\
\end{array}$} & \multirow{2}{*}{\multicolumn{3}{|c|}{ Total U }} & \multirow{3}{*}{$\begin{array}{l}{ }^{238} \mathbf{P u} \\
\text { Gp I }\end{array}$} & \multirow[b]{3}{*}{ Gp II } & \multirow{3}{*}{${ }^{239,240} \mathbf{P u}$} & \multirow[b]{3}{*}{ Gp II } & \multirow{3}{*}{$\begin{array}{r}{ }^{241} \mathrm{Am} \\
\mathbf{G p} \mathbf{I} \\
\end{array}$} & \multirow[b]{3}{*}{ Gp II } & \multirow{3}{*}{$\begin{array}{c}\text { Gross } \\
\text { Alpha } \\
\text { Gp I }\end{array}$} & \multirow[b]{3}{*}{ Gp II } & \multirow{3}{*}{$\begin{array}{c}\text { Gross } \\
\text { Beta } \\
\text { Gp I } \\
\end{array}$} & \multirow{2}{*}{\multicolumn{3}{|c|}{$\begin{array}{c}\text { Gross } \\
\text { Gamma }\end{array}$}} \\
\hline & & & & & & & & & & & & & & & & & & & & \\
\hline & & & & & & Gp II & Gp I & Gp II & & & & & & & & & & Gp II & Gp I & Gp II \\
\hline Mean (x) & 0.037 & 0.025 & 0.208 & 0.320 & 0.308 & 0.381 & 3.009 & 3.273 & 0.404 & 1.457 & 7.735 & 19.051 & 0.004 & 0.011 & 8.012 & 13.572 & 5.538 & 6.661 & 2.334 & 2.817 \\
\hline Variance $\left(\mathbf{s}^{2}\right)$ & 0.061 & 0.116 & 0.044 & 0.173 & 0.049 & 0.038 & 0.555 & 1.286 & 0.108 & 4.718 & 36.256 & 496.130 & 0.000 & 0.000 & 10.713 & 236.40 & 4.228 & 15.598 & 1.100 & 1.561 \\
\hline Count & 43 & 24 & 47 & 24 & 68 & 33 & 68 & 30 & 83 & 45 & 83 & 45 & 47 & 21 & 47 & 18 & 47 & 18 & 56 & 24 \\
\hline Diff & 0.000 & & 0.000 & & 0.000 & & 0.000 & & 0.000 & & 0.000 & & 0.000 & & 0.000 & & 0.000 & & 0.000 & \\
\hline df & 1 & & 1 & & 1 & & 1 & & 1 & & 1 & & 1 & & 1 & & 1 & & 1 & \\
\hline KW-stat & 0.003 & & 0.400 & & 5.402 & & 3.803 & & 25.281 & & 20.421 & & 16.338 & & 3.305 & & 0.965 & & 4.063 & \\
\hline KW-table & 3.840 & & 3.840 & & 3.840 & & 3.840 & & 3.840 & & 3.840 & & 3.840 & & 3.840 & & 3.840 & & 3.840 & \\
\hline Significant & No & & No & & Yes & & No & & Yes & & Yes & & Yes & & No & & No & & No & \\
\hline
\end{tabular}

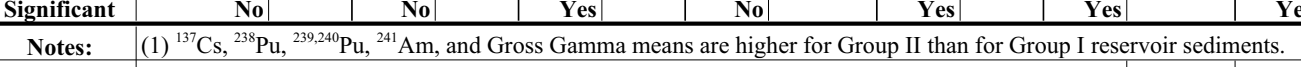

(2) Highlighted values are statistically significant; two-tail value reported for KW-table statistic. 


\section{APPENDIX C \\ COMPUTER CODE LISTING}

This appendix presents the computer code that was used to create and plot the normal, lognormal, and square-root-transformed probability plots of the raw and normalized data that are depicted in Appendices D and $\mathrm{E}$. This program is written in the MATLAB language (version 5.3, release 11). It was executed using the PC version of MATLAB; however, other platform versions are available from the vendor (The MathWorks, Inc., Natick, MA; http://www.mathworks.com). Note that the program instructions are executed in an m-file mode that requires the Statistics toolbox. The user must provide the input data file (Xi), which is a onedimensional matrix containing the analyte radioactivity values in column one.

The input data are normalized within the program using the following procedure. First, the mean value of the input file is subtracted from each value in the file. Then this resultant is divided by the standard deviation of the input values. This procedure yields a new file having a new mean of approximately zero and a new standard deviation of approximately one. This new file is then linearly transformed into all positive values by adding a constant to each new value. This constant equals the sum of the absolute value of the minimum number in the new file and the new standard deviation. This transform was required so that all data values in the original input file would be plotted on the lognormal probability plot. This transformation does not change the general shape of the original data distribution; however, the original data values are changed to dimensionless values in the probability plots. Note that the original data file sometimes contains negative values for certain radionuclides as explained in the report text. Hence, this procedure was required to ensure that all transformed data values would be plotted in the graphs.

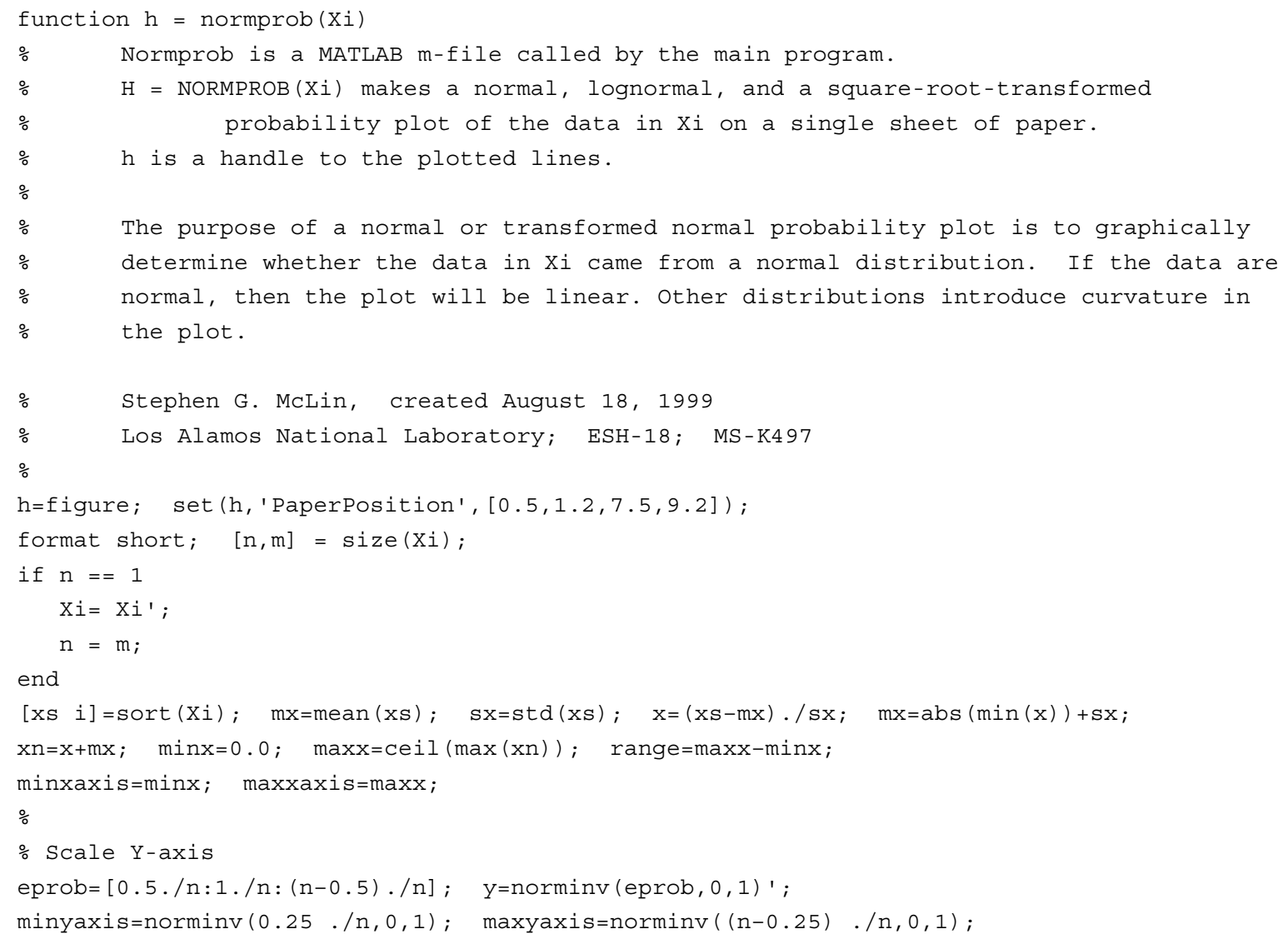




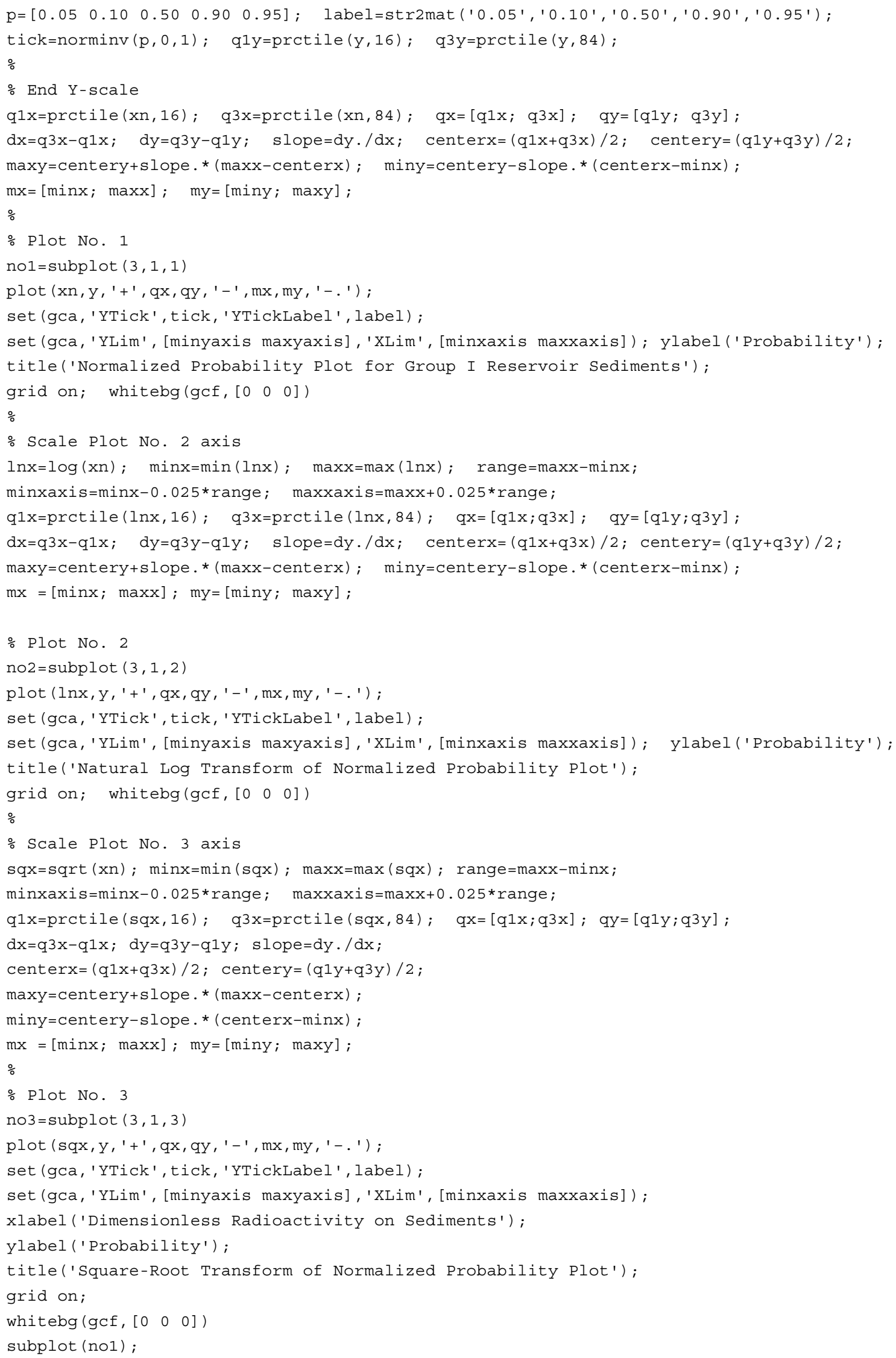


APPENDIX D

PROBABILITY PLOTS FOR GROUP I RIVER SEDIMENTS 
Normal Probability Plot for Group I River Sediments

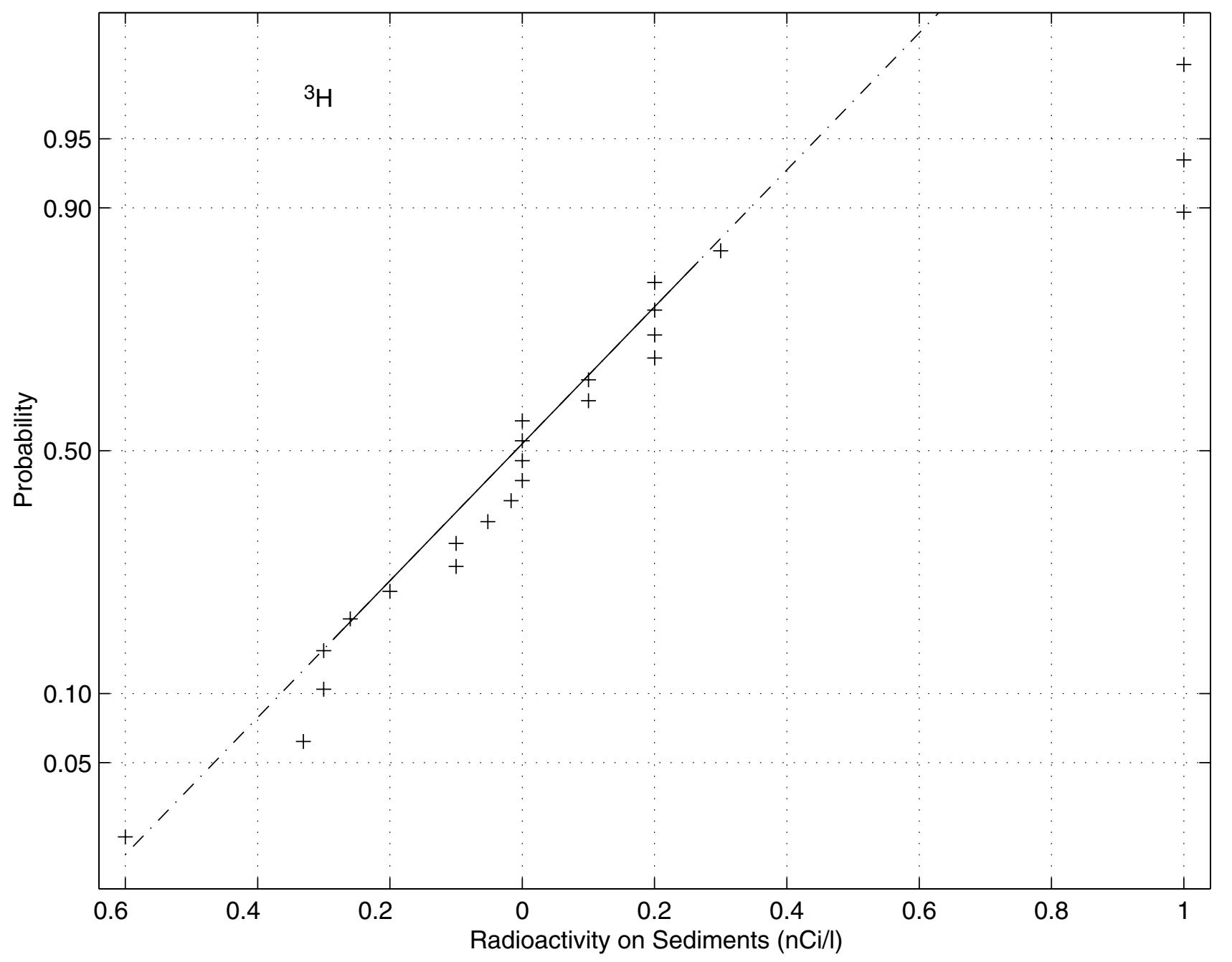

Fig. D-1. Probability plot for tritium in Group I river sediments. 

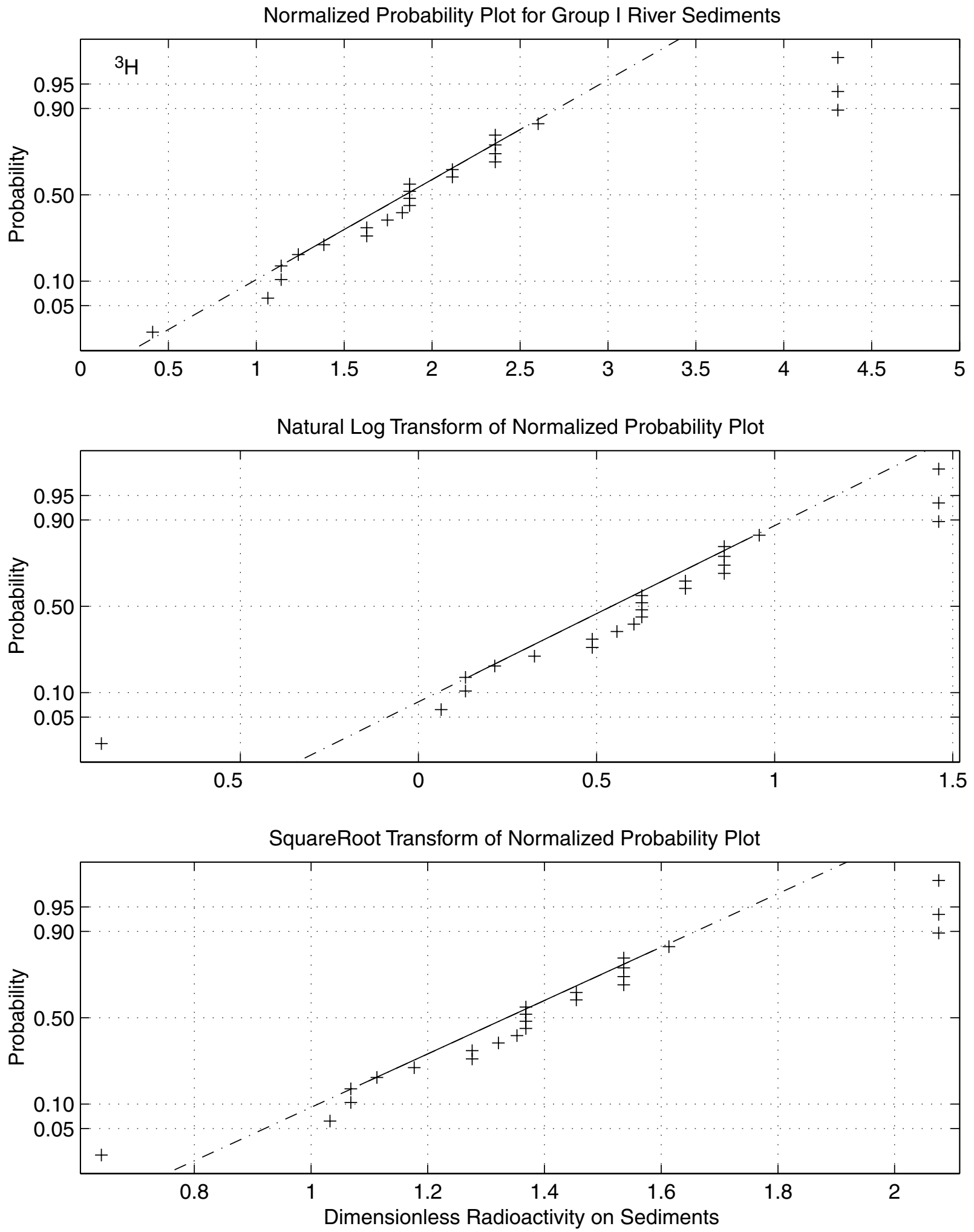

Fig. D-2. Normalized plots for tritium in Group I river sediments. 


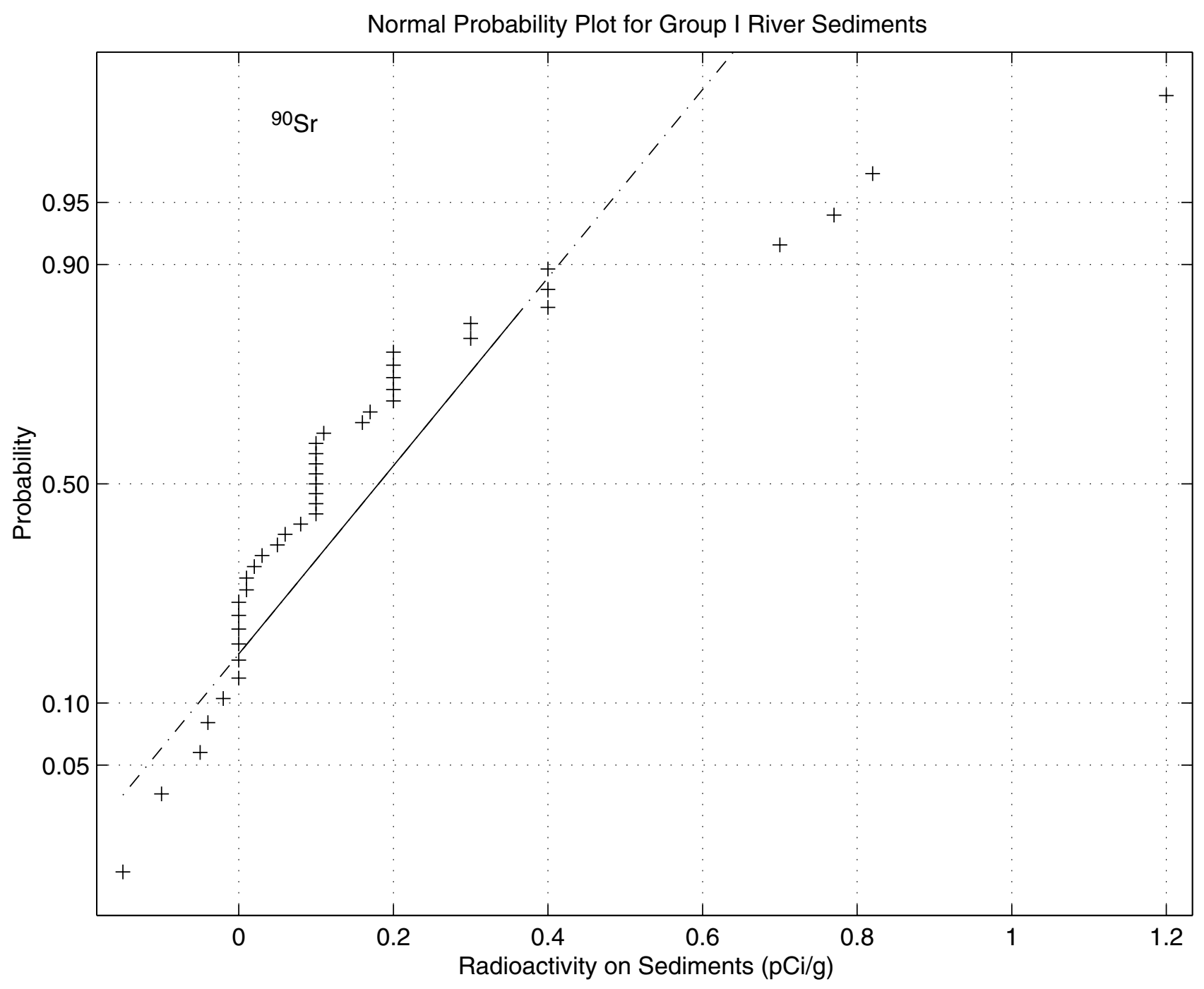

Fig. D-3. Probability plot for strontium-90 in Group I river sediments. 
Normalized Probability Plot for Group I River Sediments

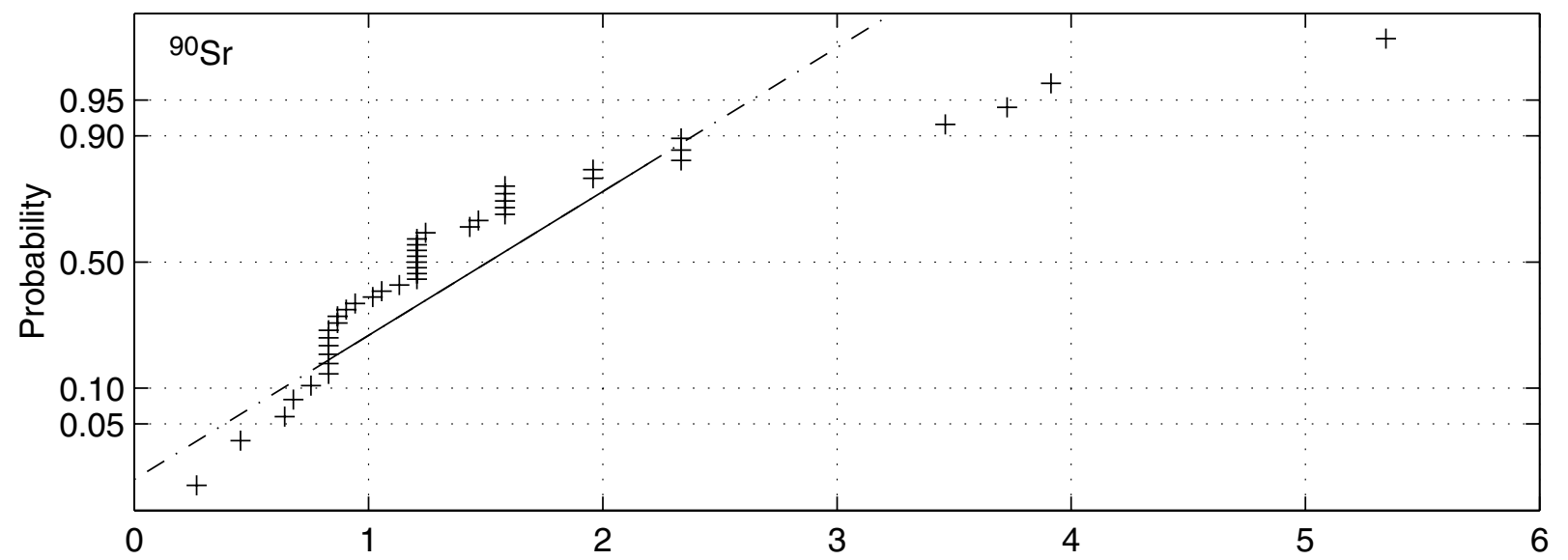

Natural Log Transform of Normalized Probability Plot

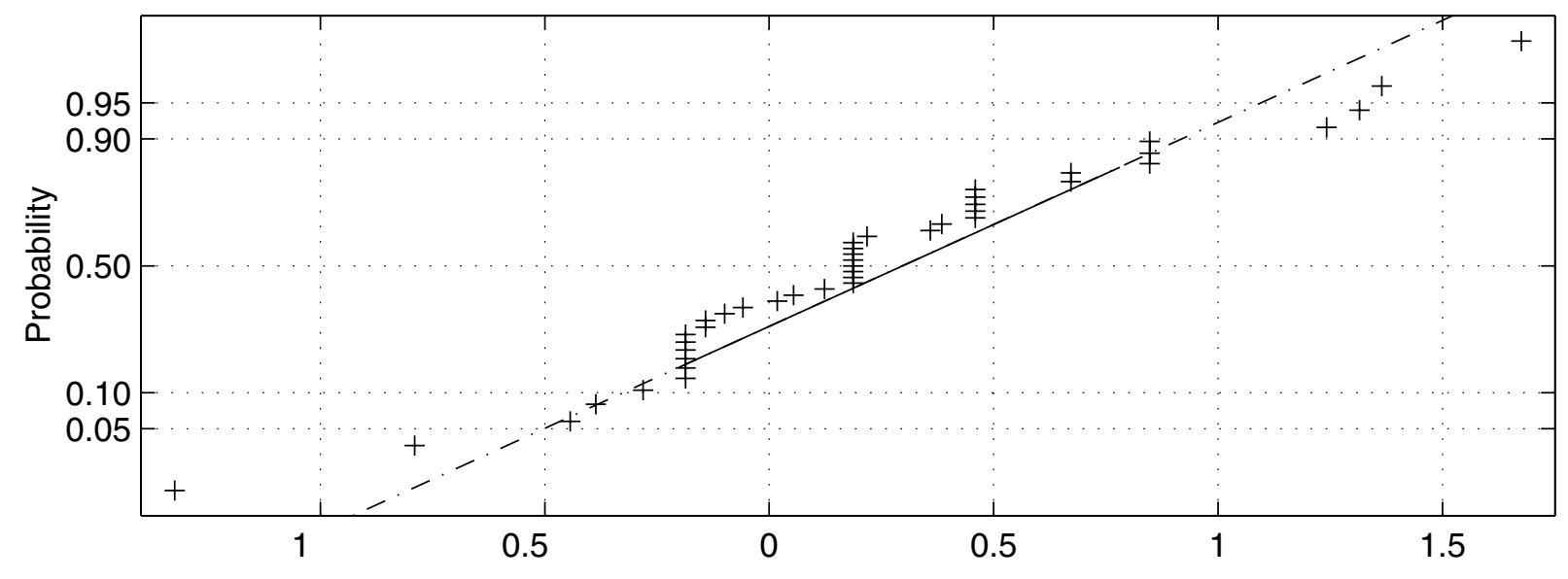

Square-Root Transform of Normalized Probability Plot

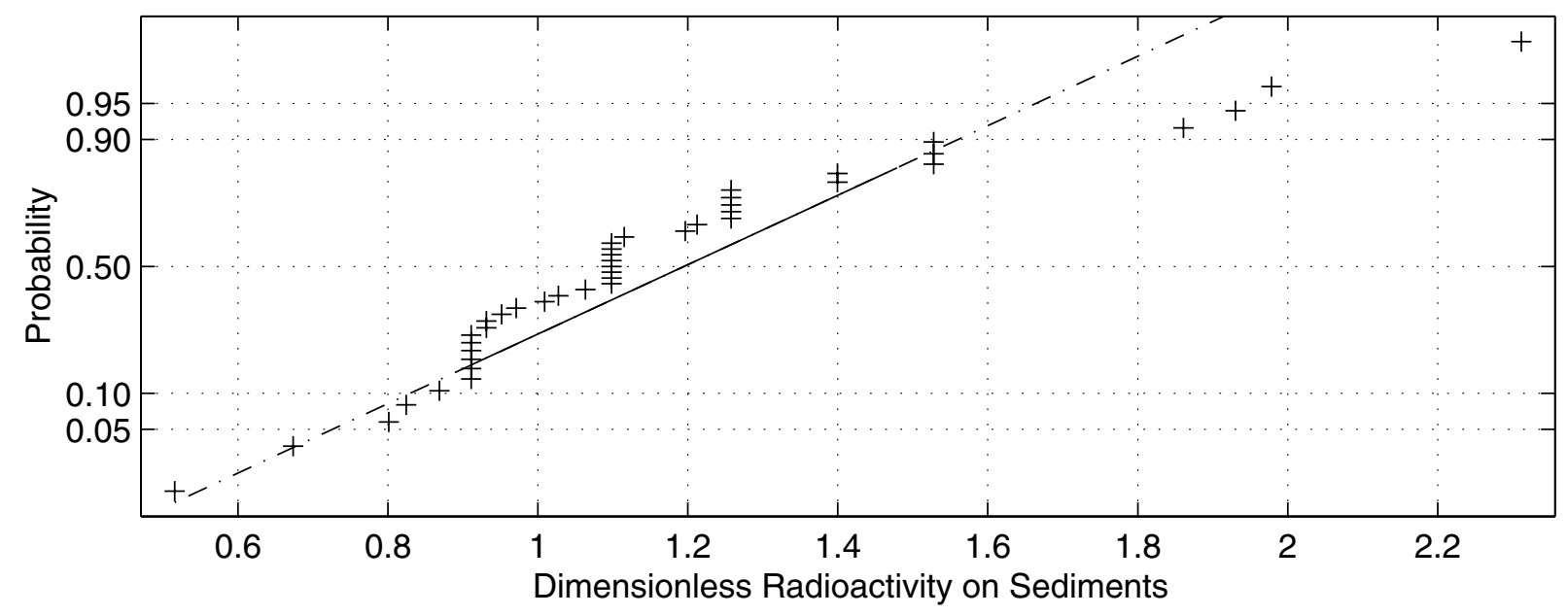

Fig. D-4. Normalized plots for strontium-90 in Group I river sediments. 


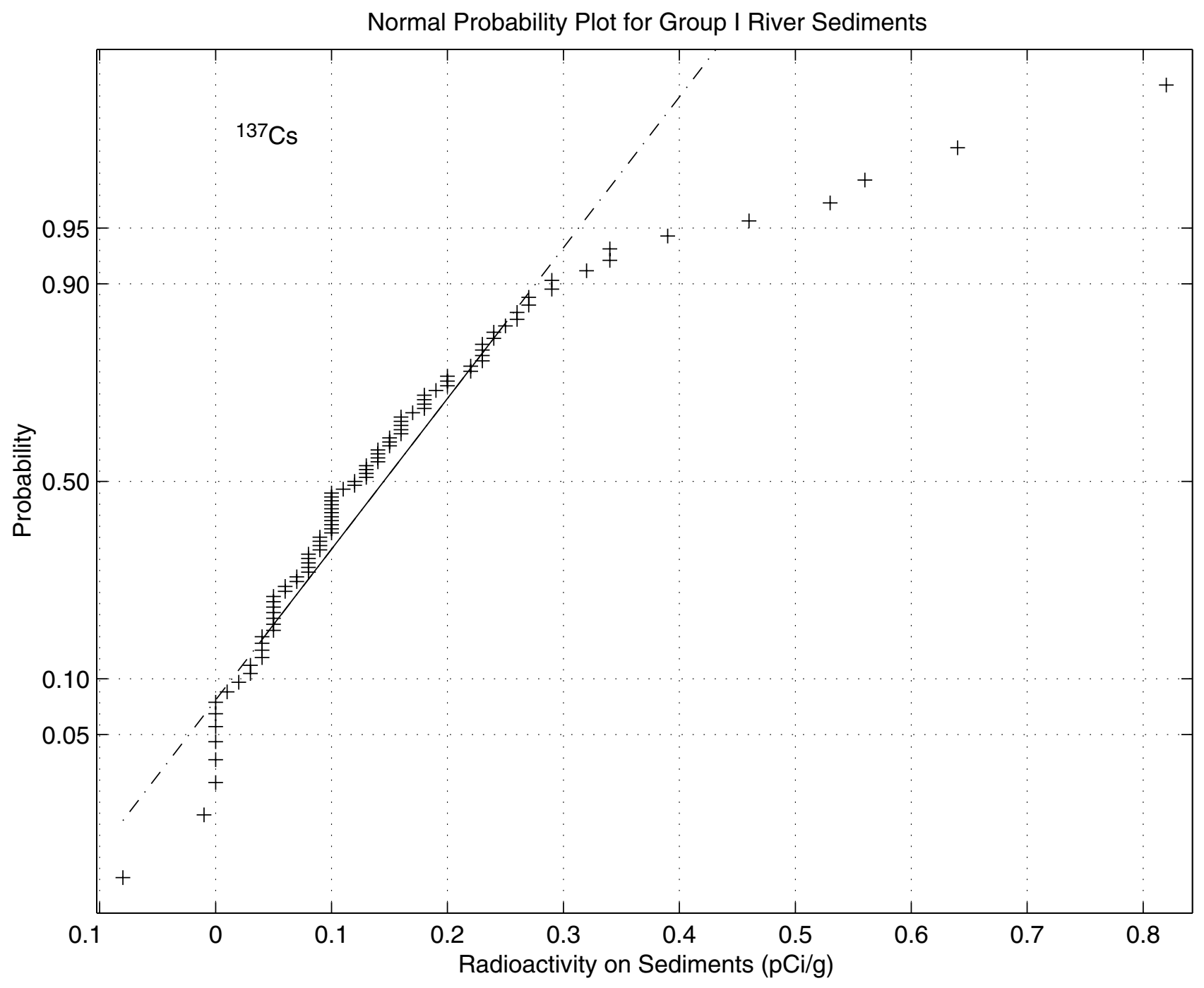

Fig. D-5. Probability plot for cesium-137 in Group I river sediments. 


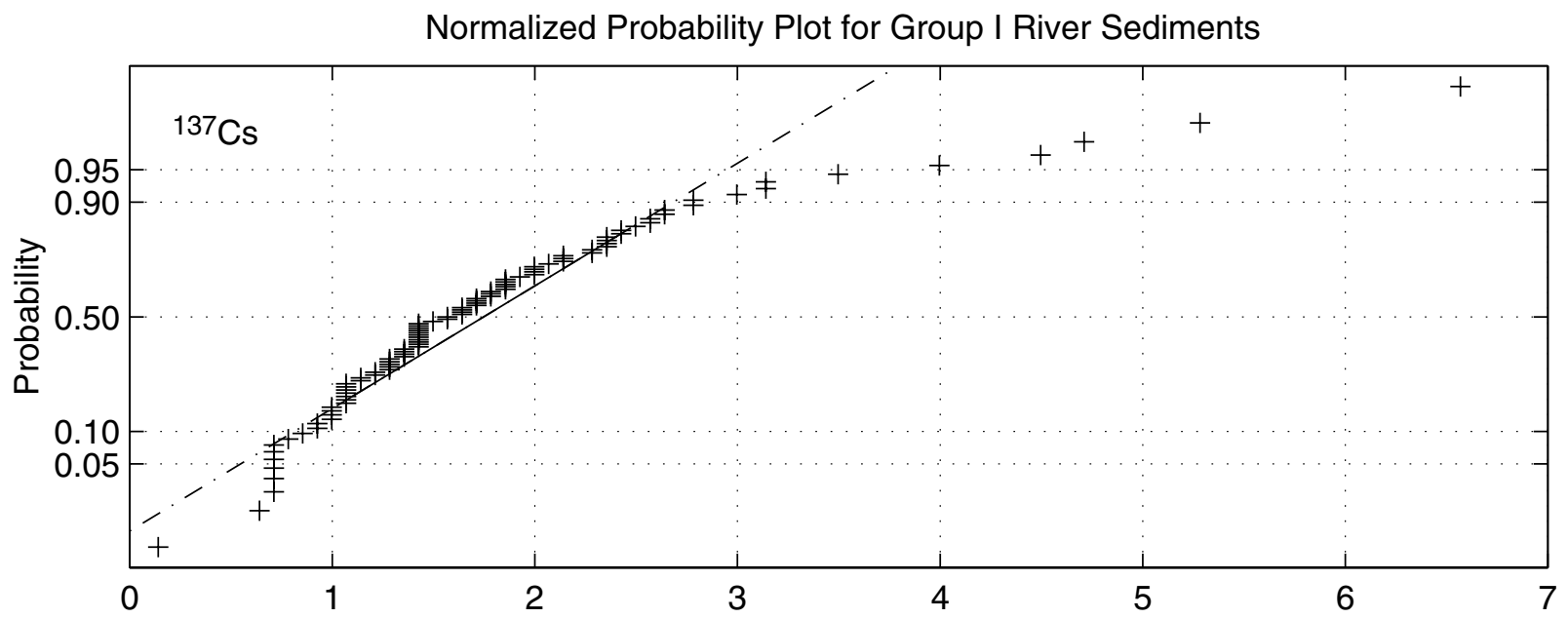

Natural Log Transform of Normalized Probability Plot

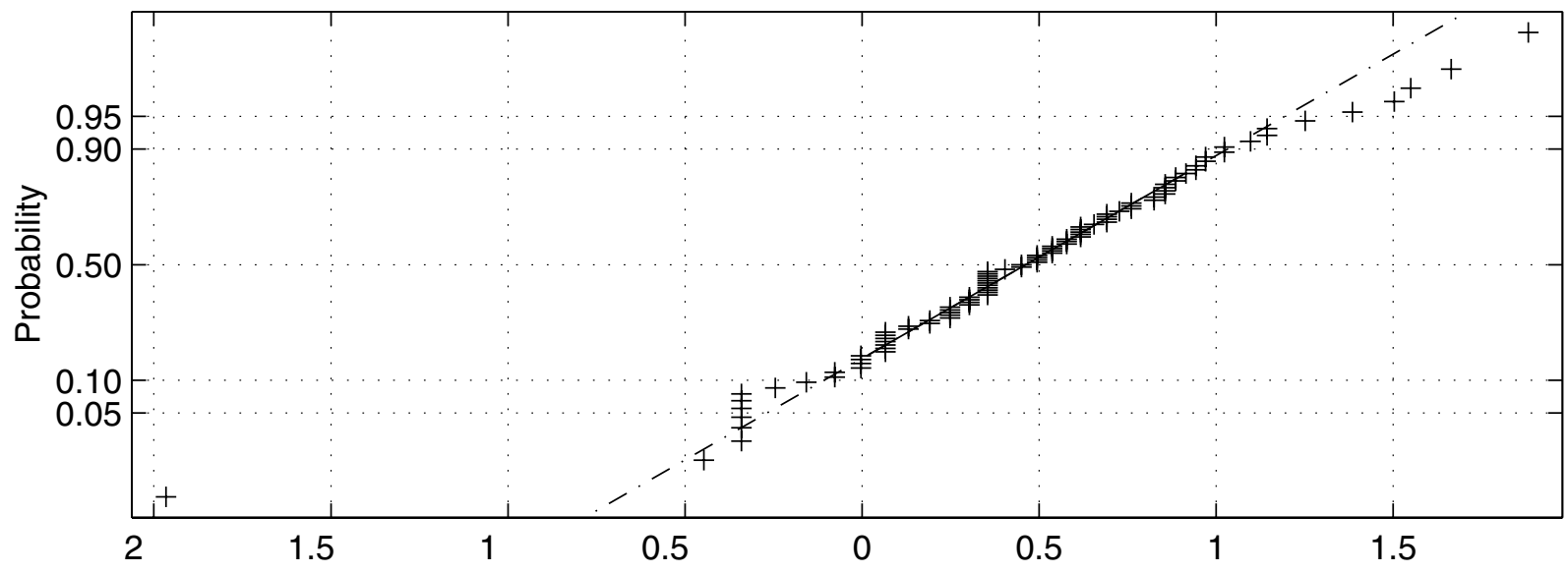

Square-Root Transform of Normalized Probability Plot

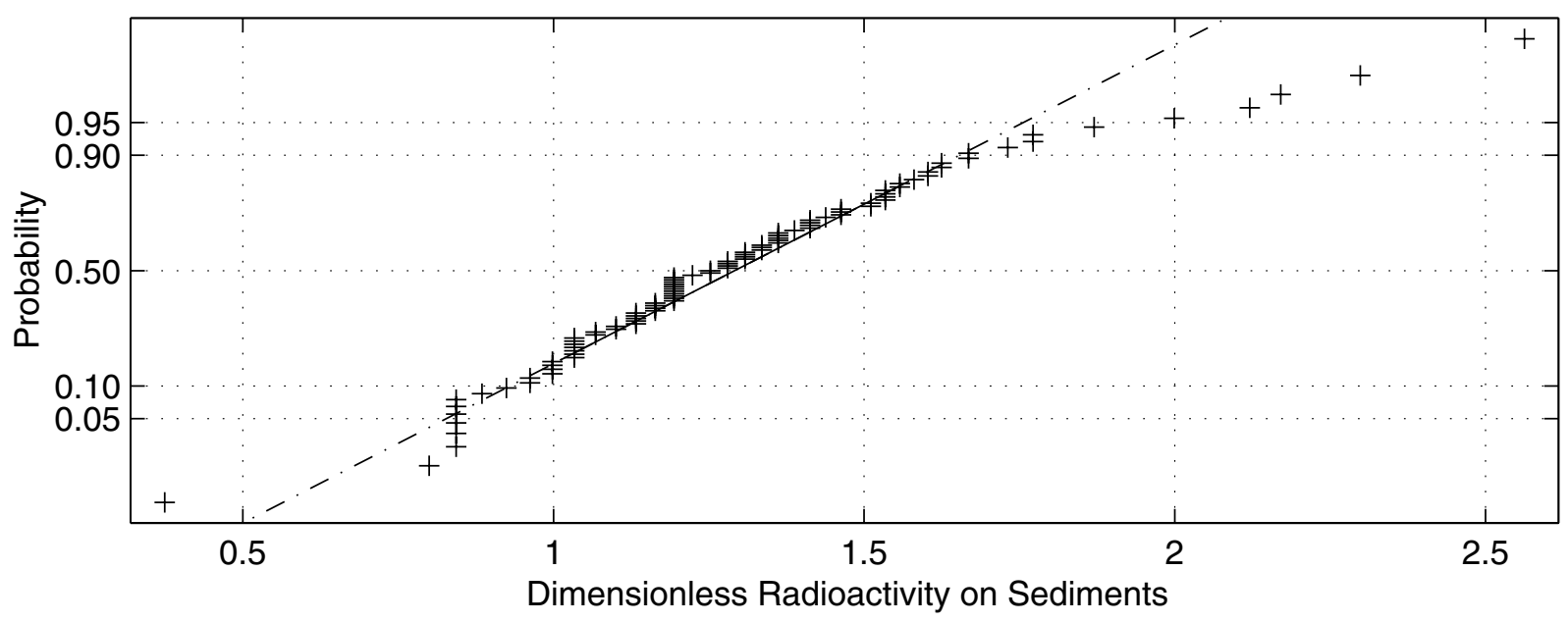

Fig. D-6. Normalized plots for cesium-137 in Group I river sediments. 


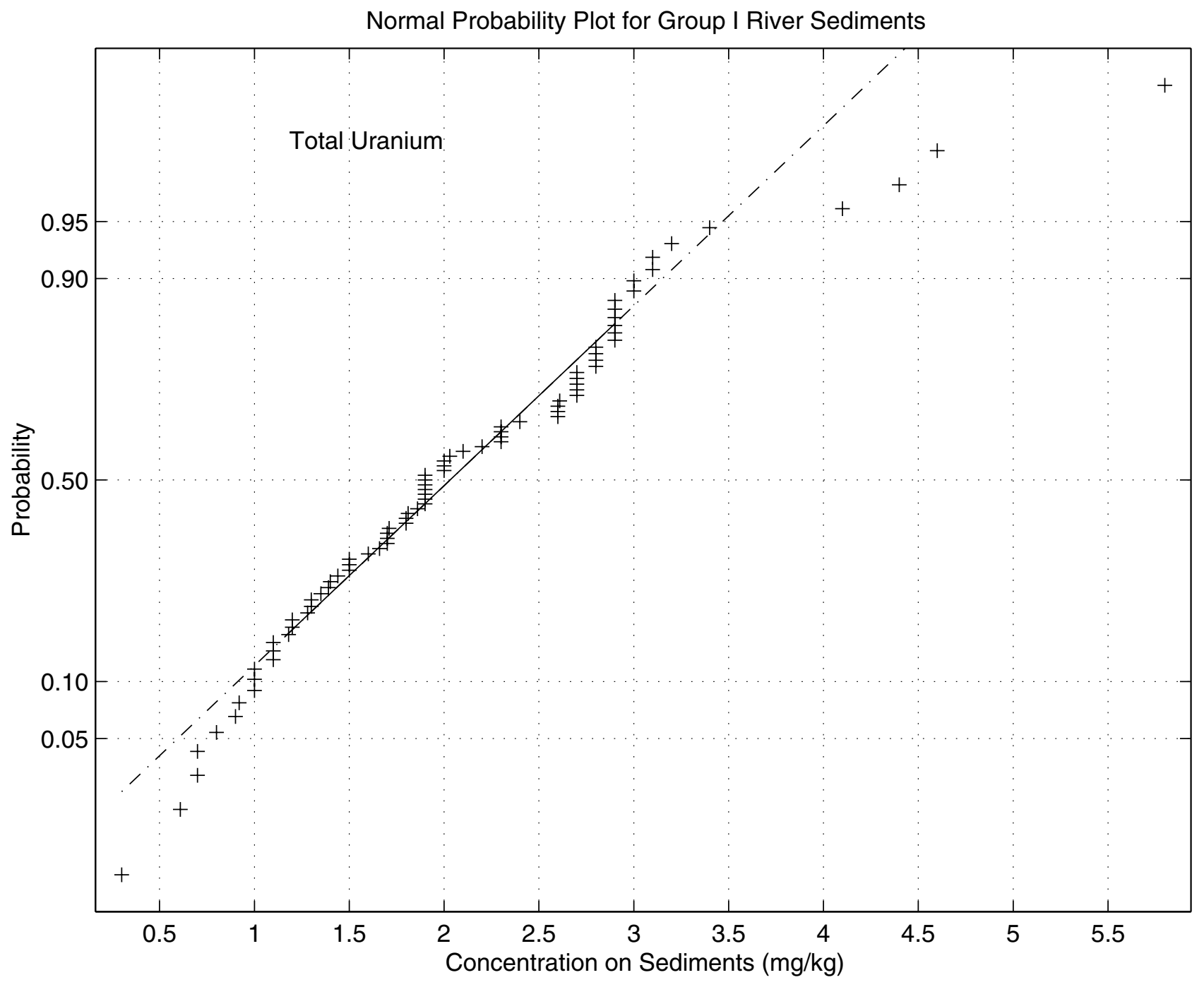

Fig. D-7. Probability plot for total uranium in Group I river sediments. 


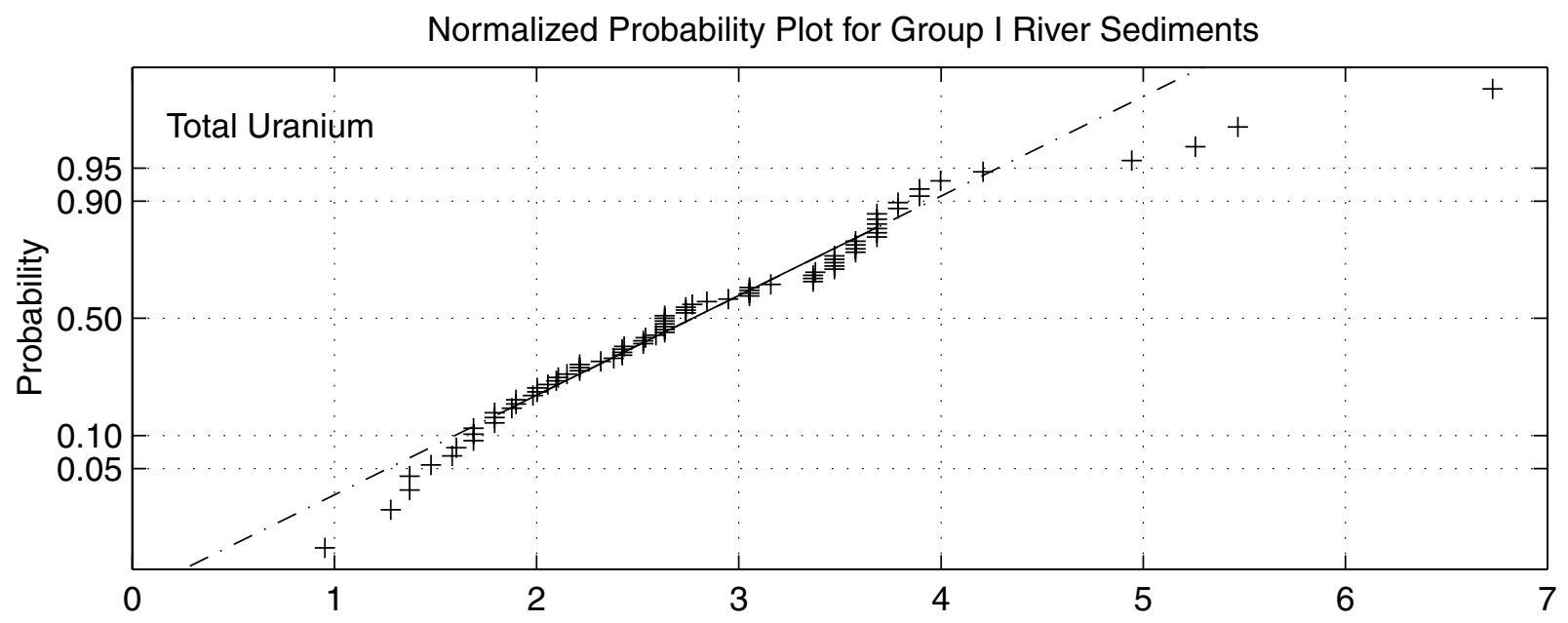

Natural Log Transform of Normalized Probability Plot

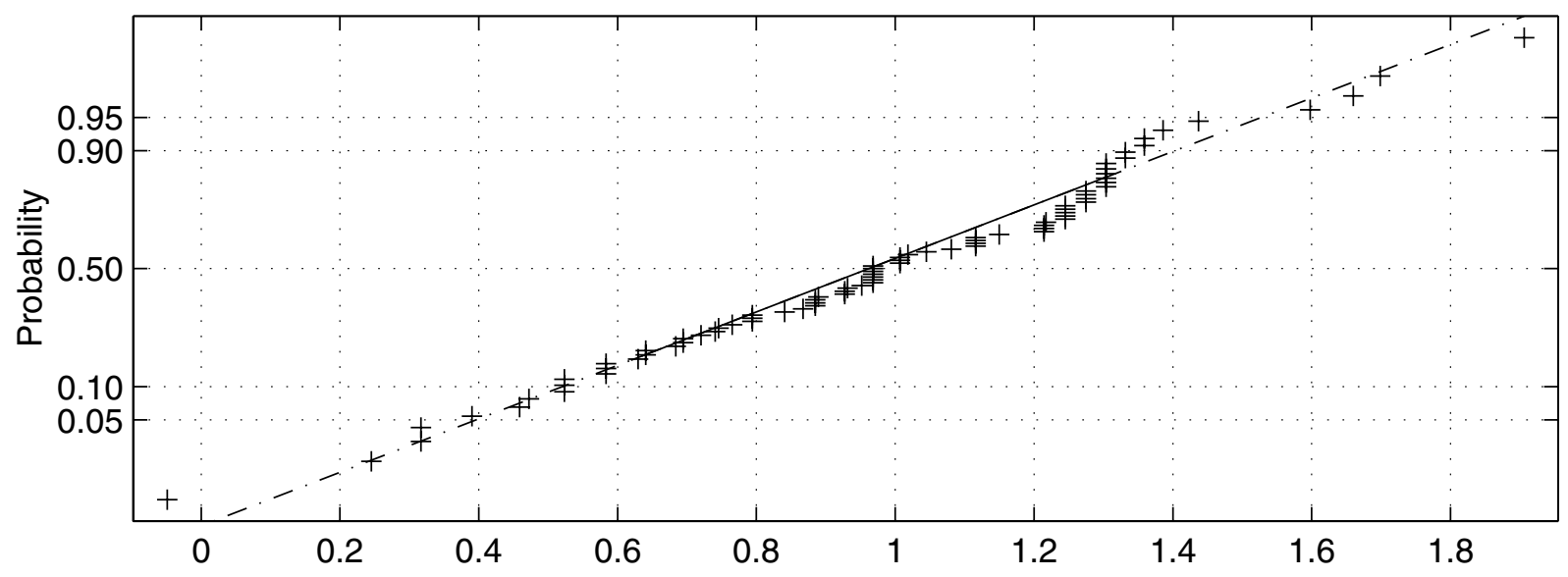

Square-Root Transform of Normalized Probability Plot

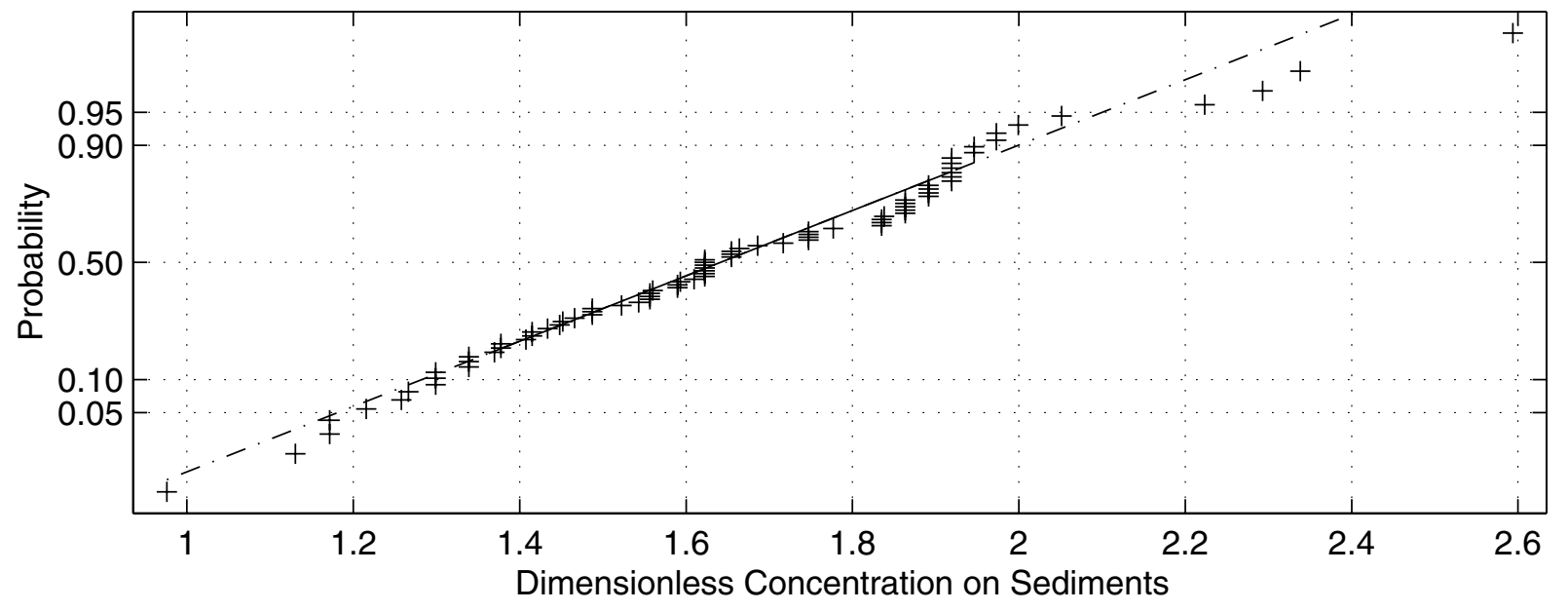

Fig. D-8. Normalized plots for total uranium in Group I river sediments. 


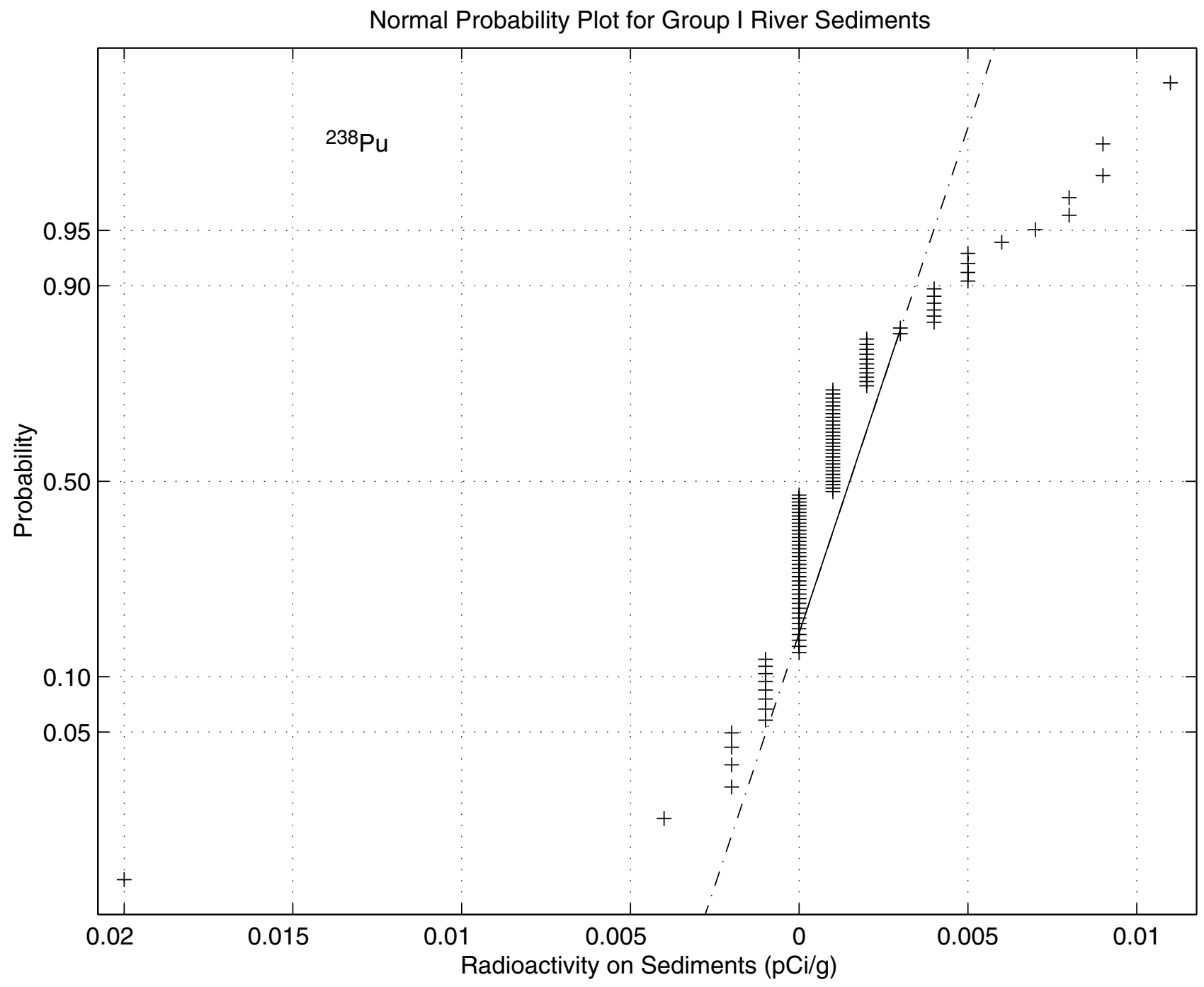

Fig. D-9. Probability plot for plutonium-238 in Group I river sediments. 
Normalized Probability Plot for Group I River Sediments

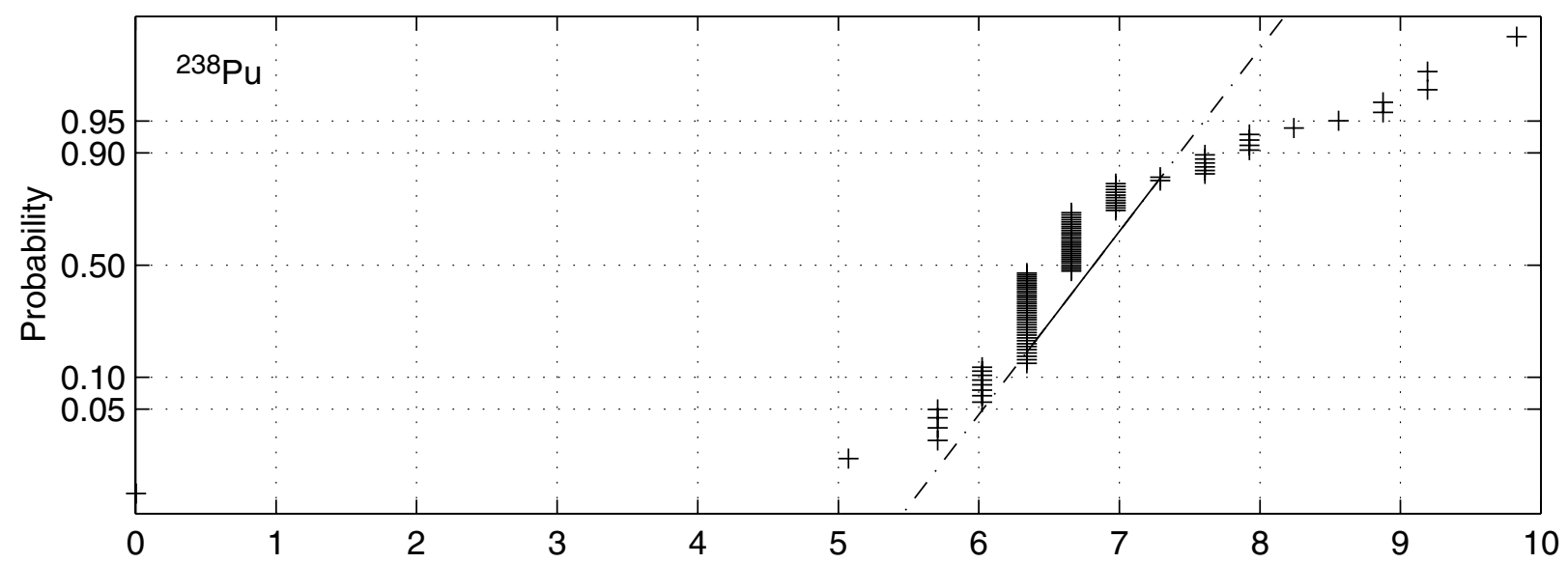

Natural Log Transform of Normalized Probability Plot

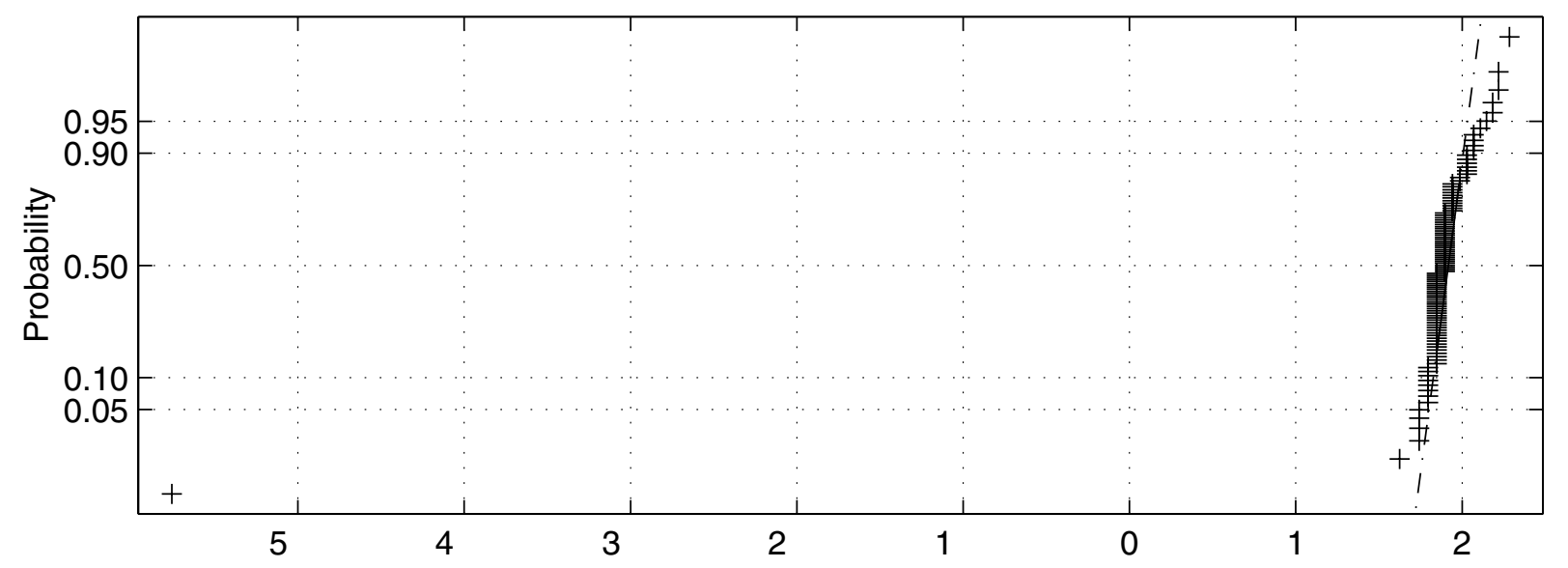

Square-Root Transform of Normalized Probability Plot

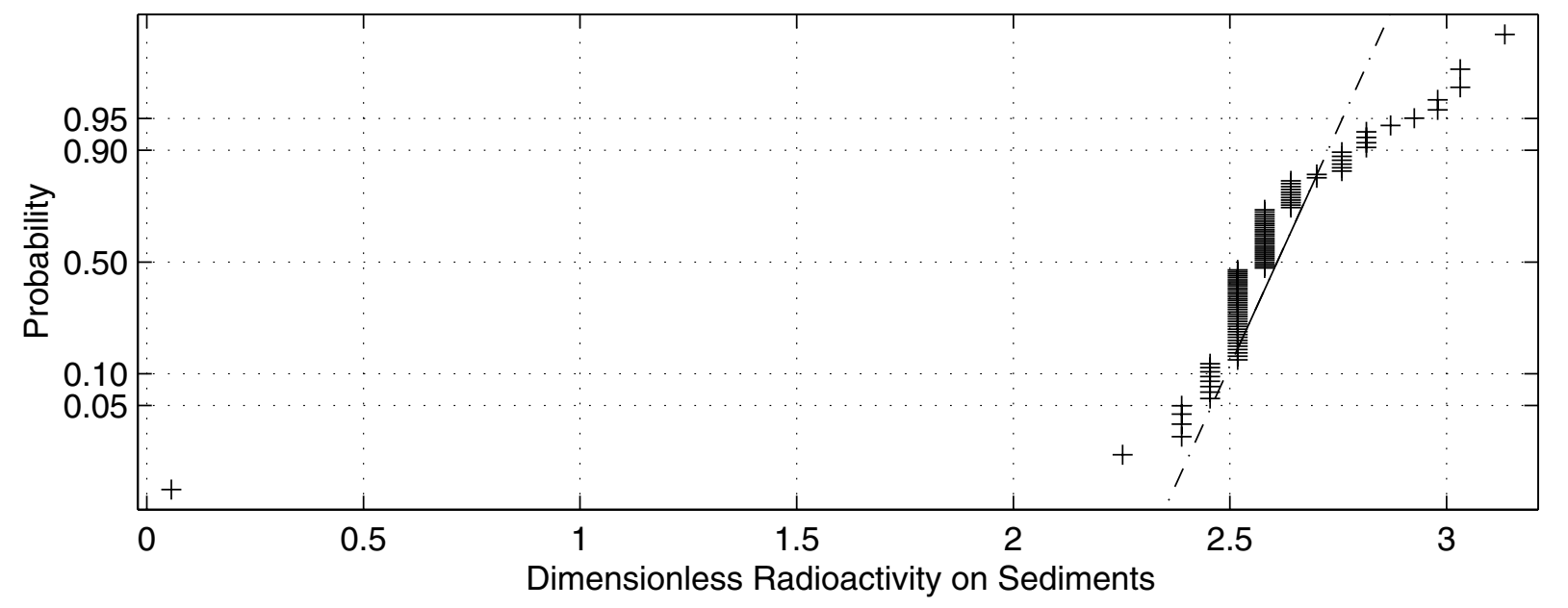

Fig. D-10. Normalized plots for plutonium-238 in Group I river sediments. 


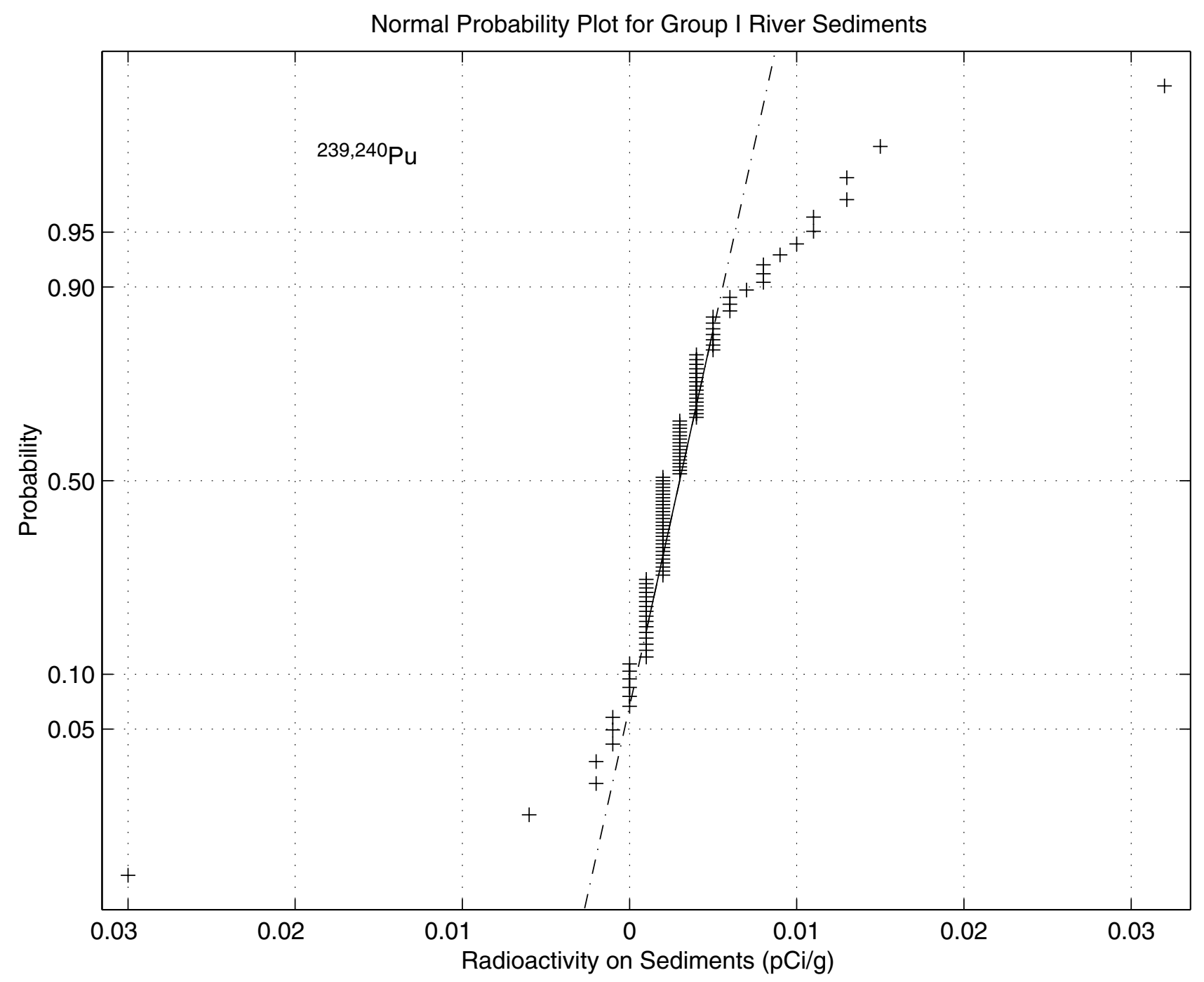

Fig. D-11. Probability plot for plutonium-239,-240 in Group I river sediments. 

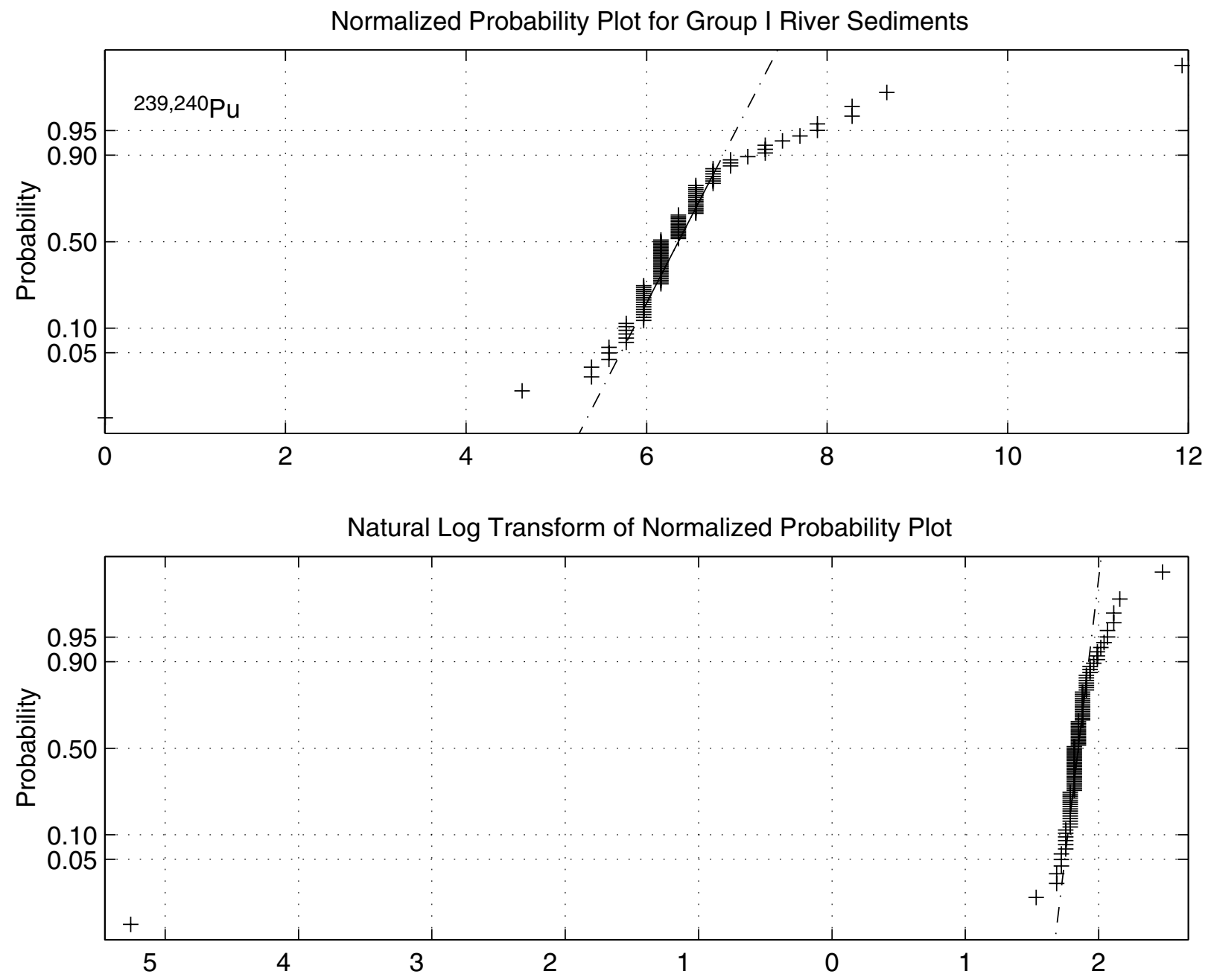

Square-Root Transform of Normalized Probability Plot

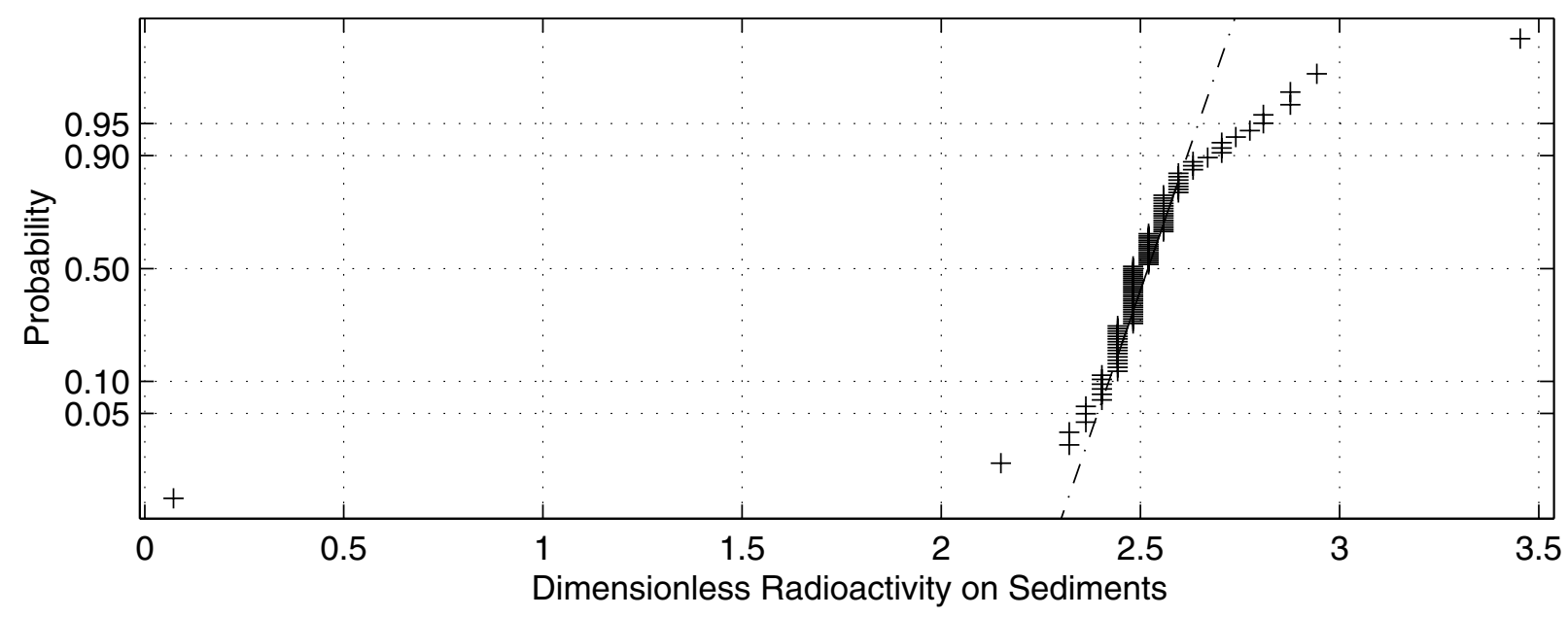

Fig. D-12. Normalized plots for plutonium-239,-240 in Group I river sediments. 
Normal Probability Plot for Group I River Sediments

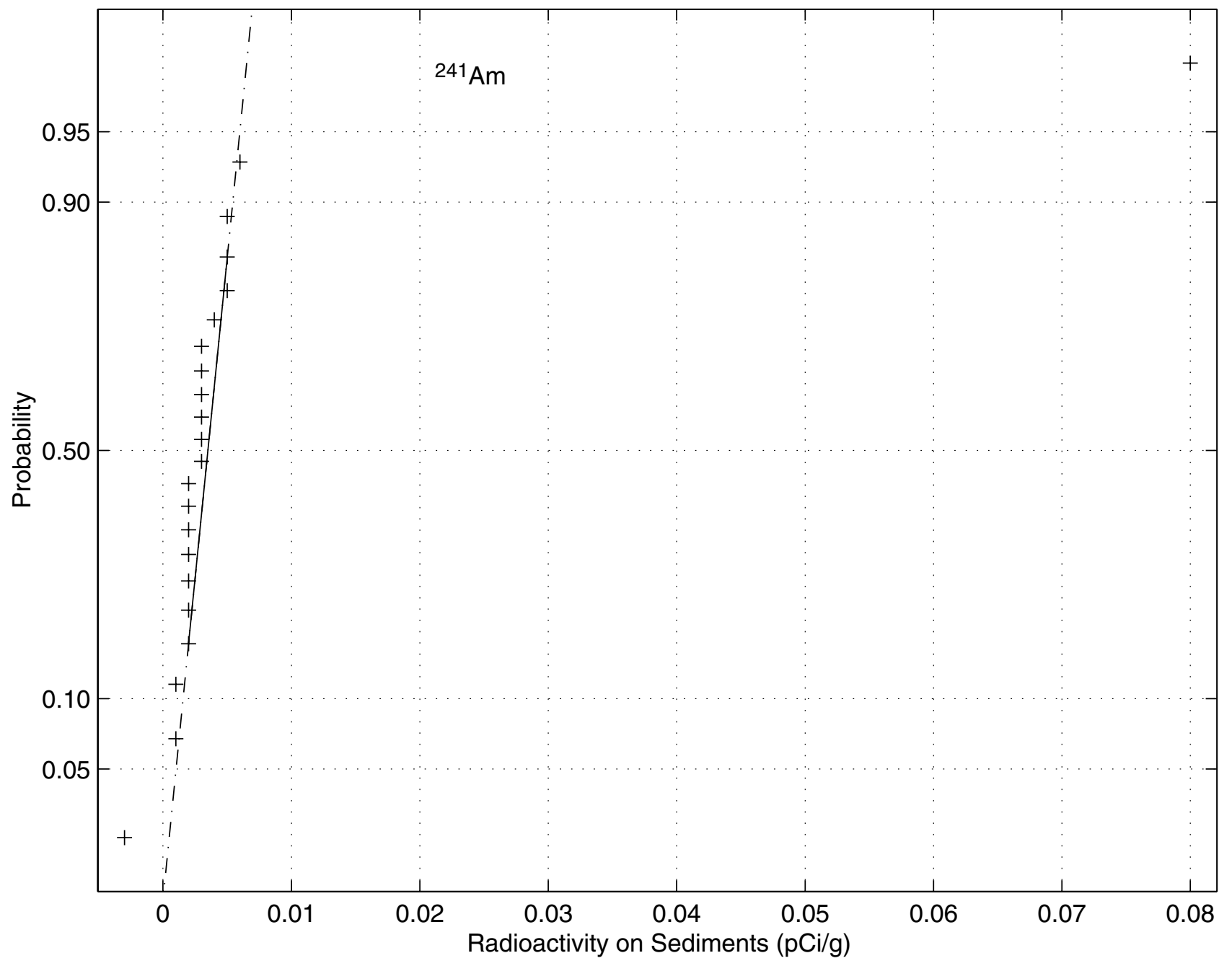

Fig. D-13. Probability plot for americium-241 in Group I river sediments. 


\section{Normalized Probability Plot for Group I River Sediments}

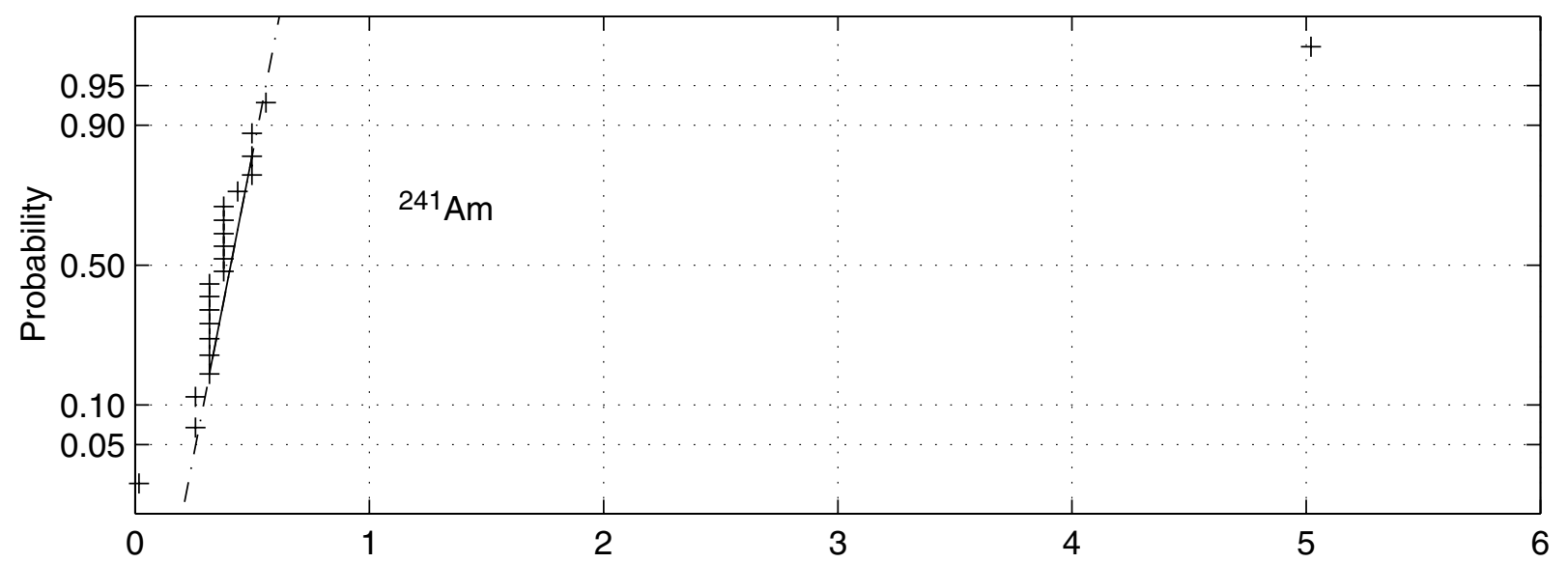

Natural Log Transform of Normalized Probability Plot

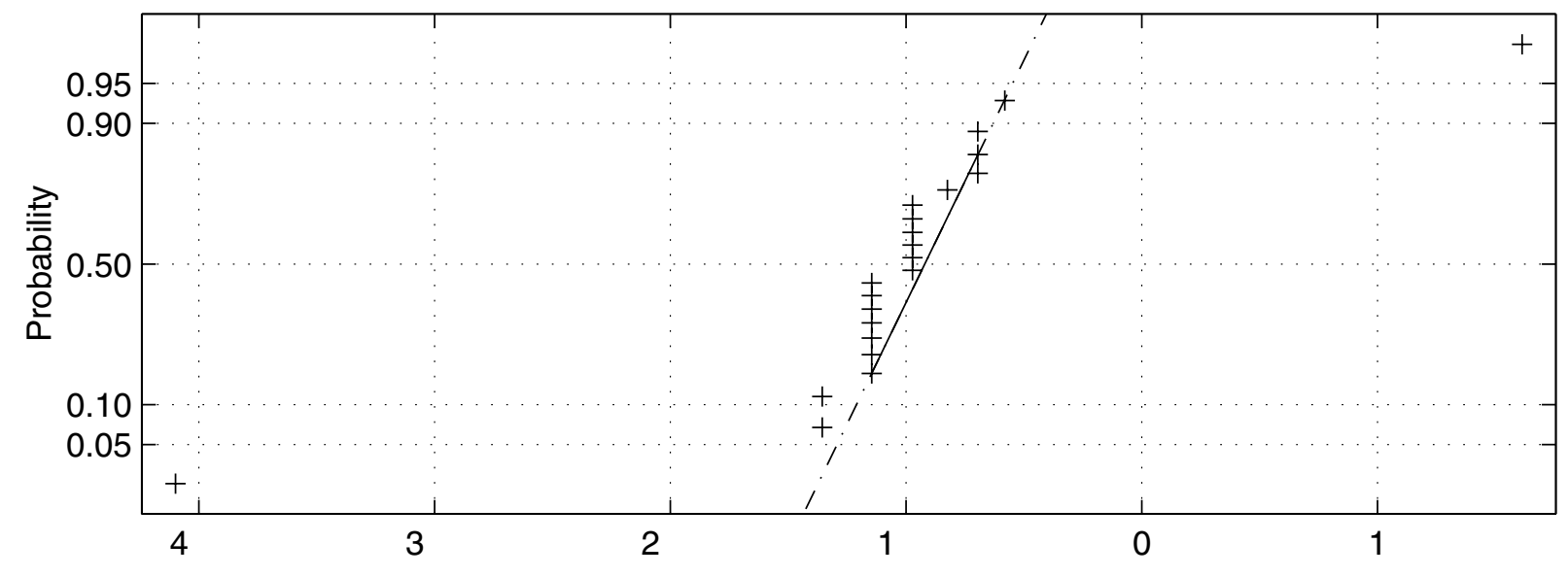

Square-Root Transform of Normalized Probability Plot

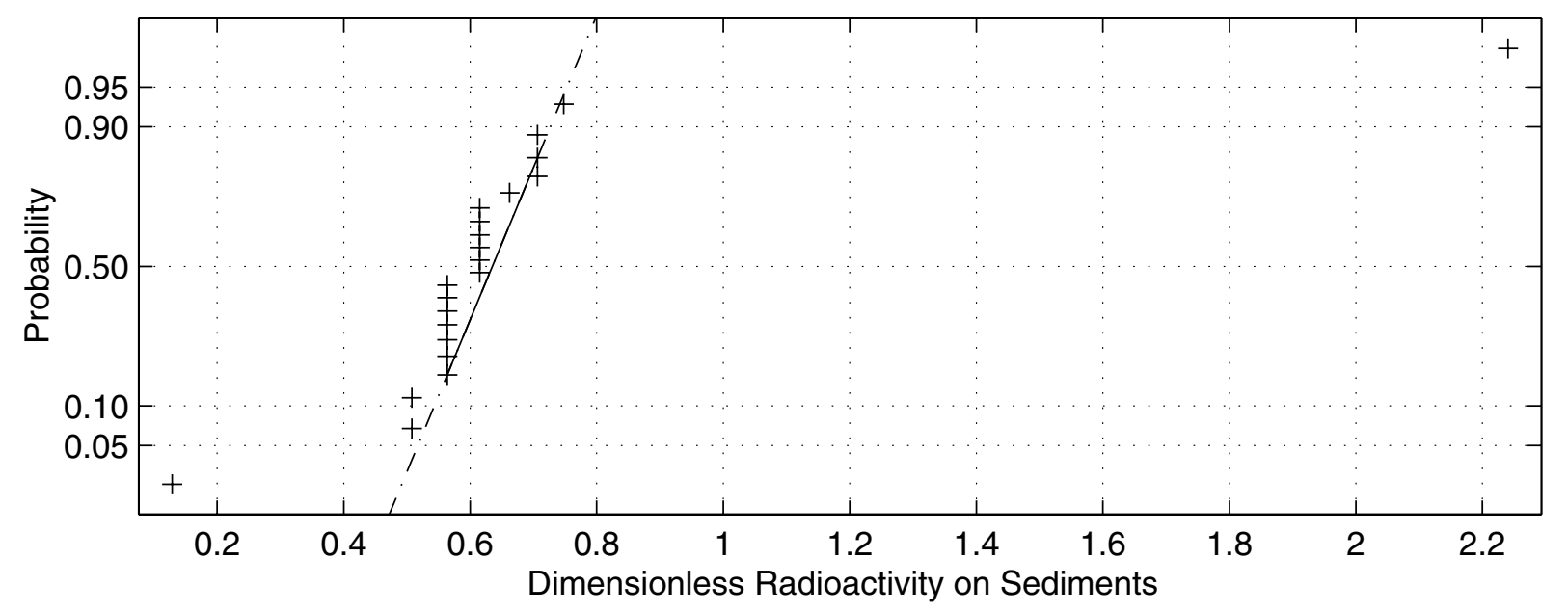

Fig. D-14. Normalized plots for americium-241 in Group I river sediments. 


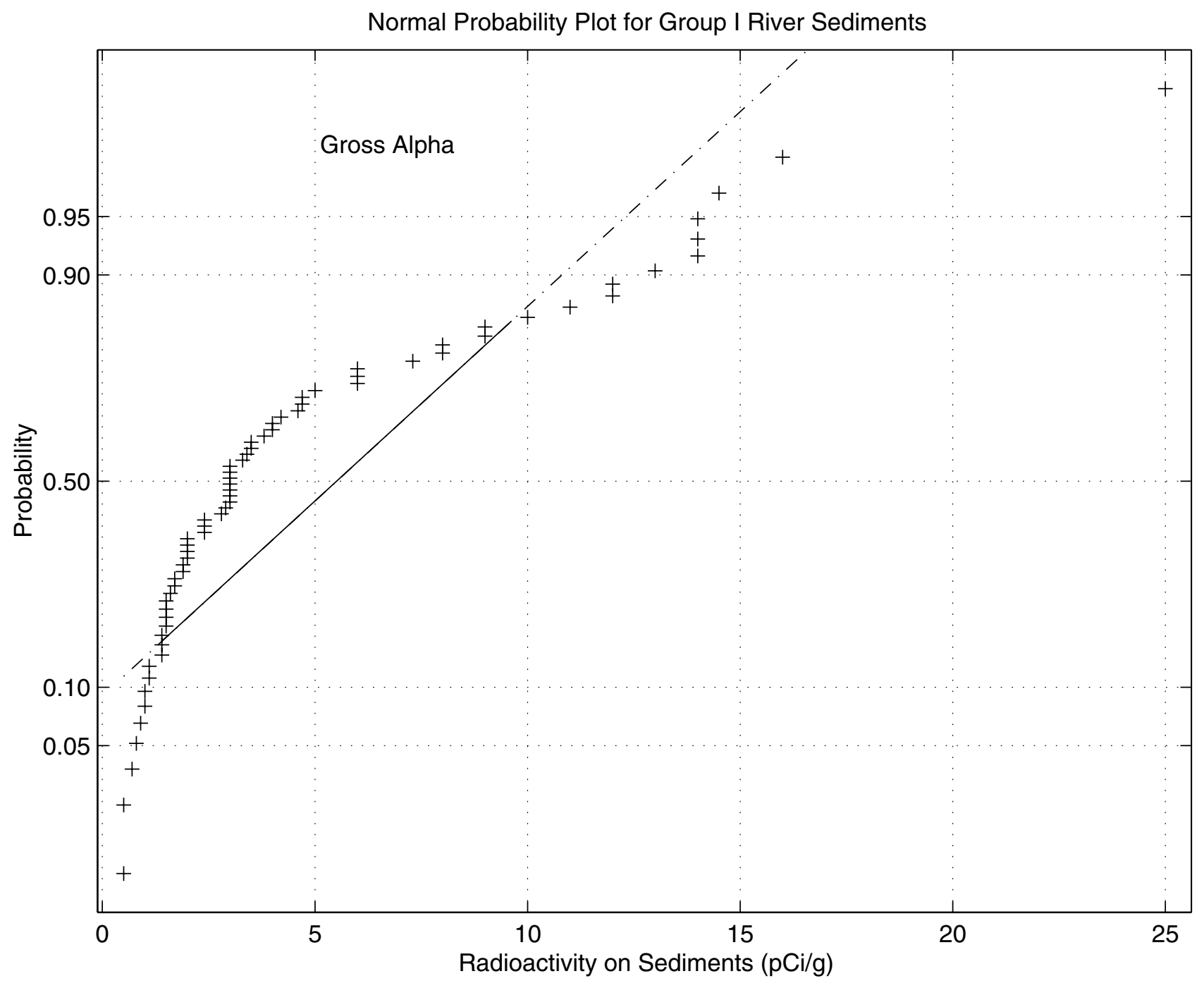

Fig. D-15. Probability plot for gross alpha in Group I river sediments. 


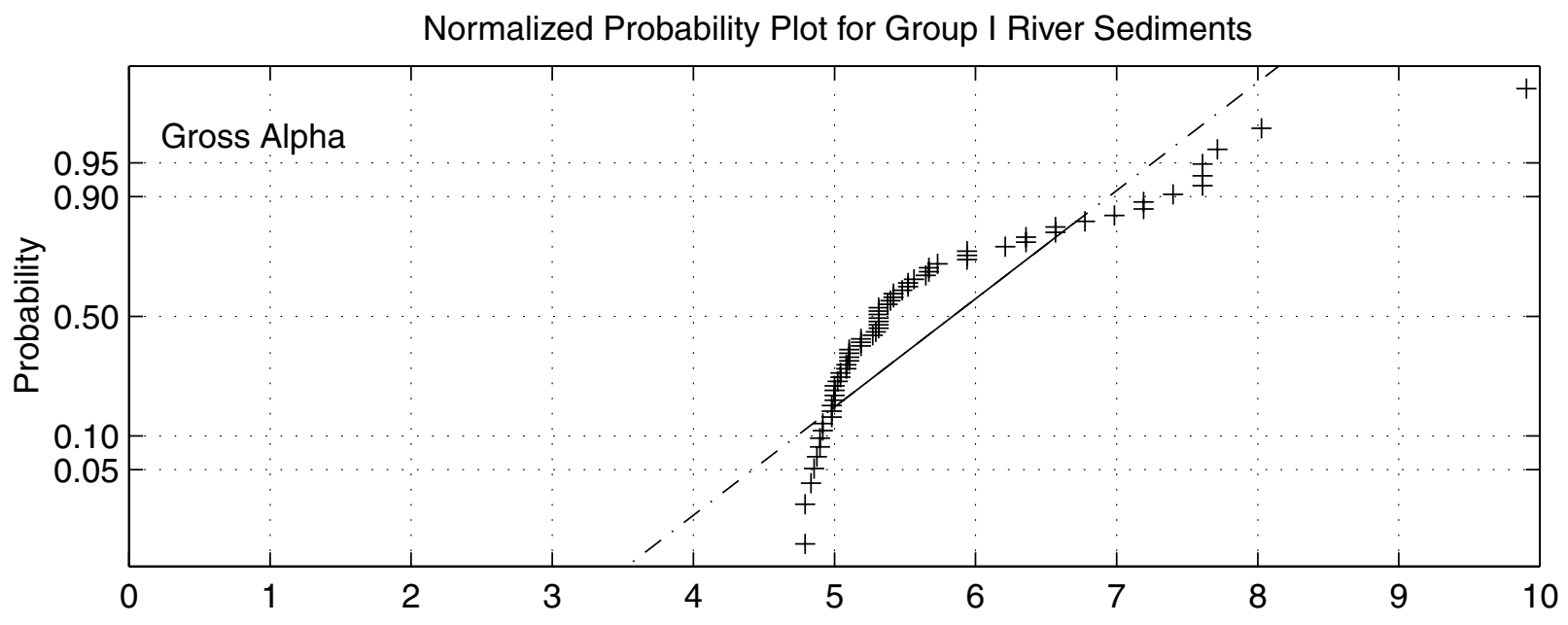

Natural Log Transform of Normalized Probability Plot

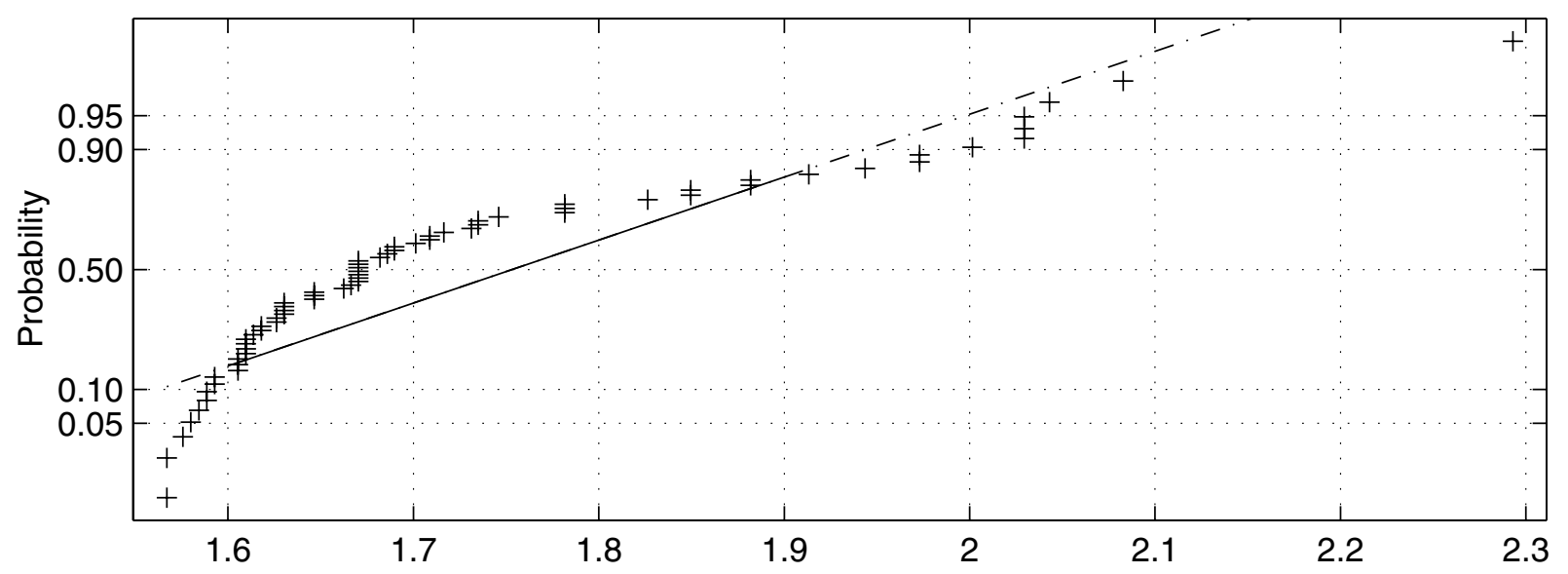

Square-Root Transform of Normalized Probability Plot

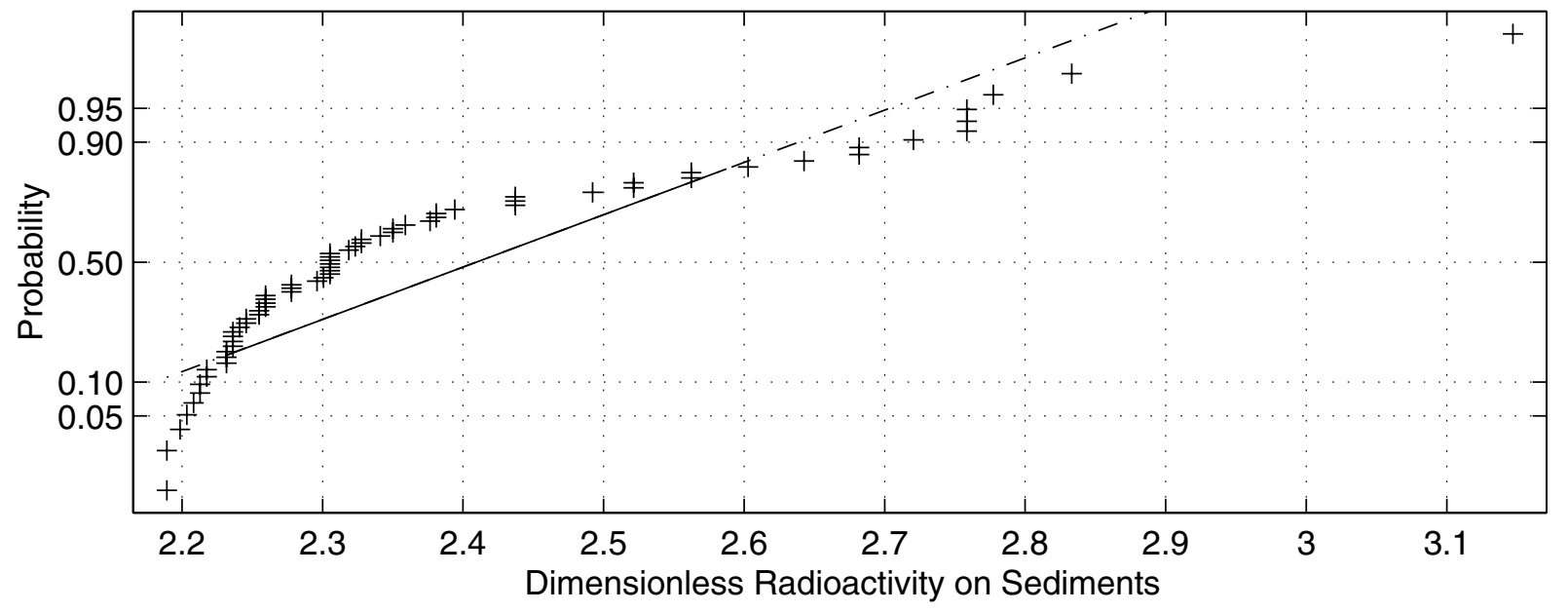

Fig. D-16. Normalized plots for gross alpha in Group I river sediments. 


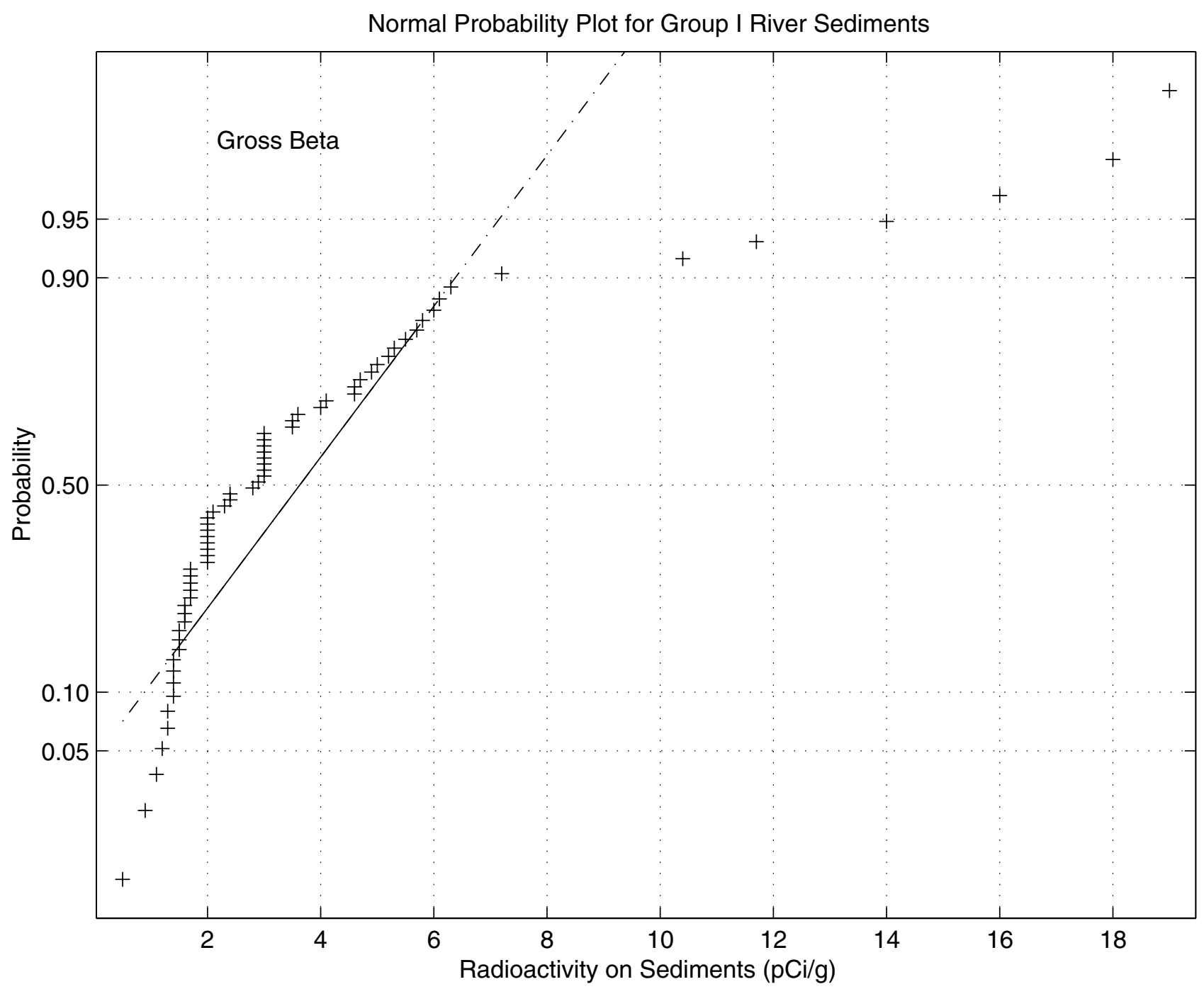

Fig. D-17. Probability plot for gross beta in Group I river sediments. 


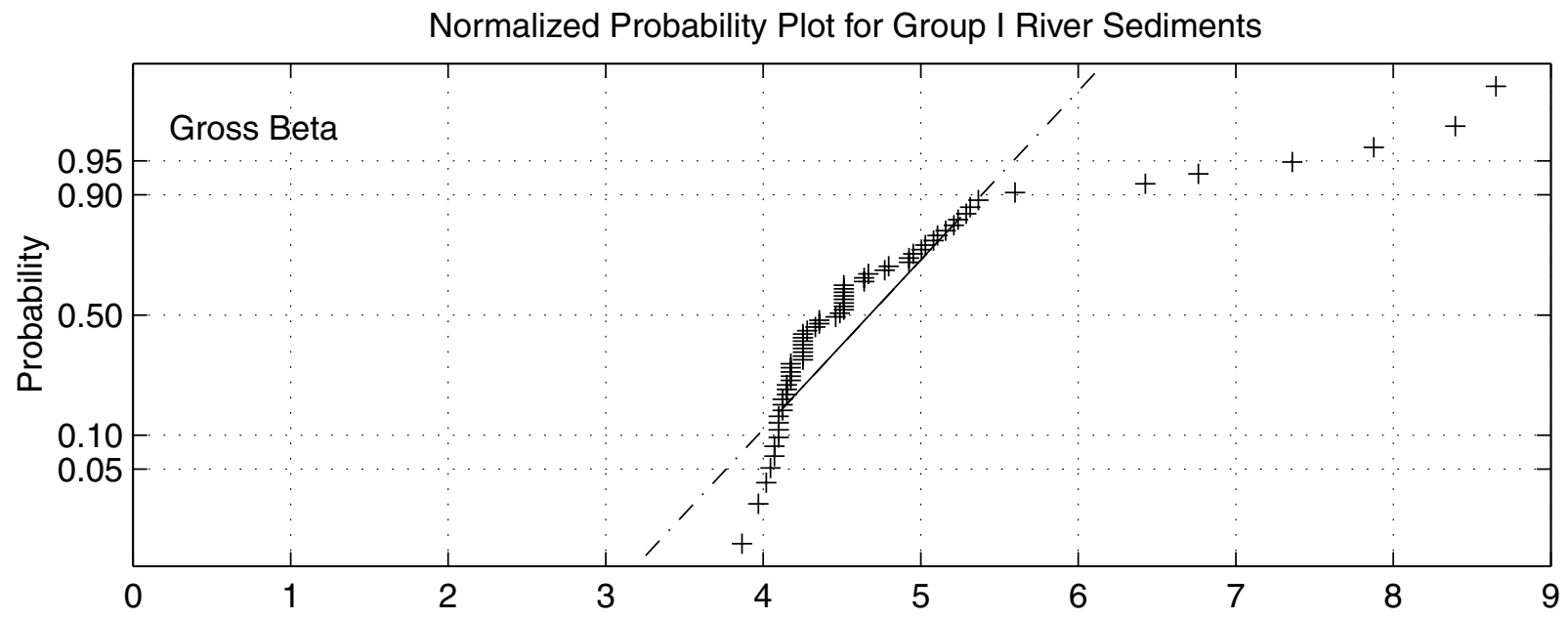

Natural Log Transform of Normalized Probability Plot

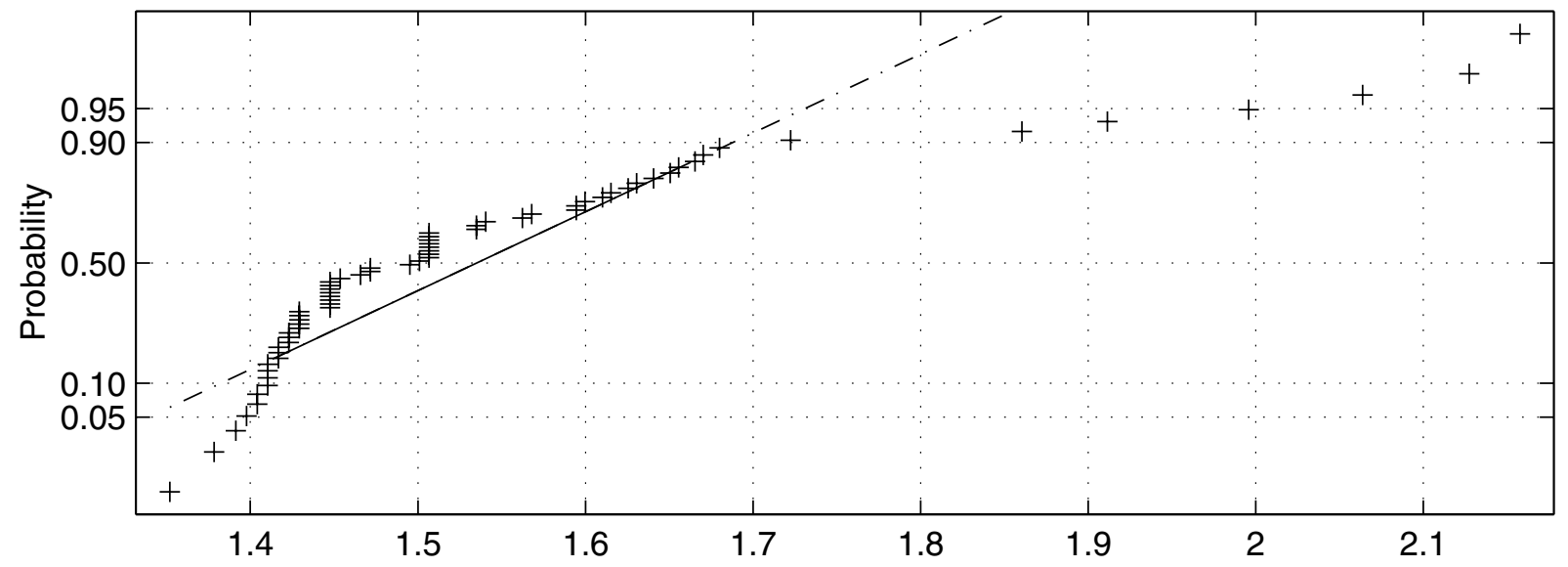

Square-Root Transform of Normalized Probability Plot

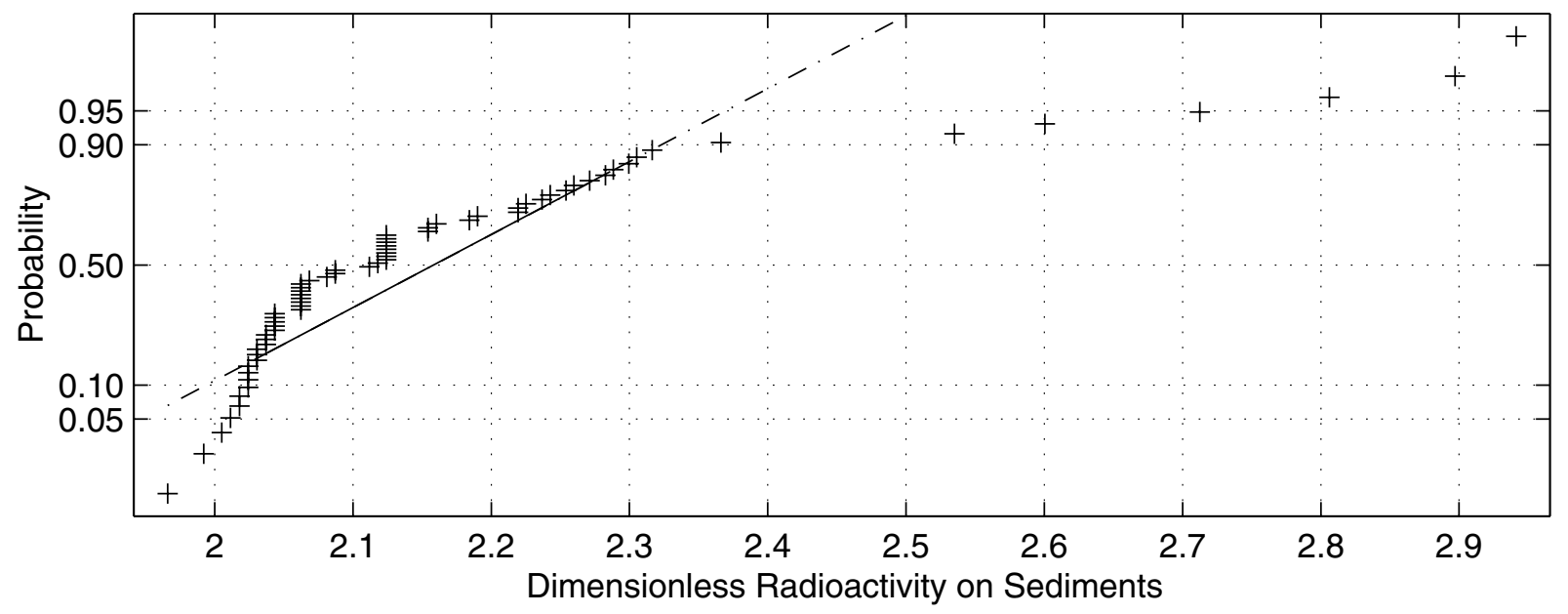

Fig. D-18. Normalized plots for gross beta in Group I river sediments. 


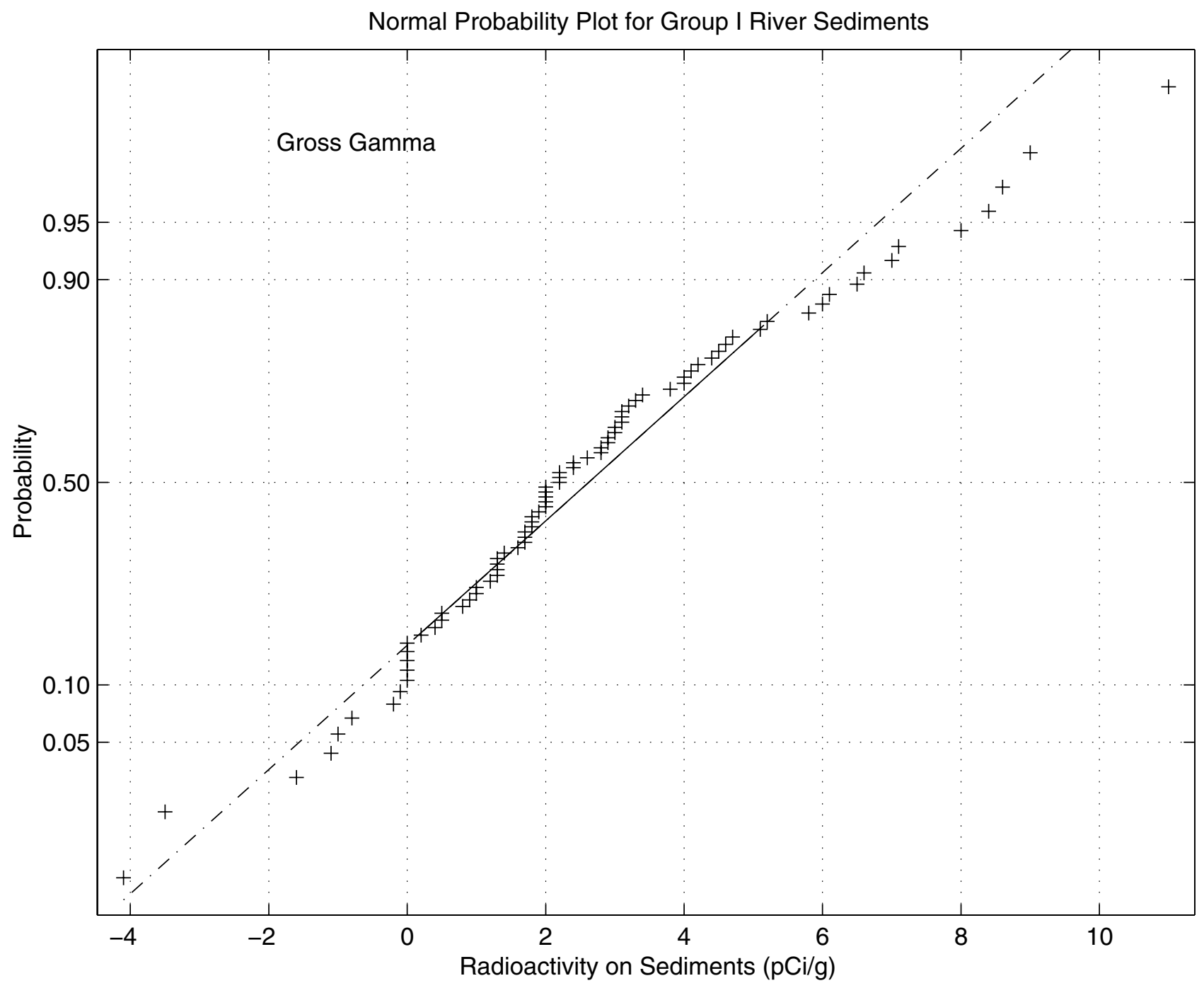

Fig. D-19. Probability plot for gross gamma in Group I river sediments. 


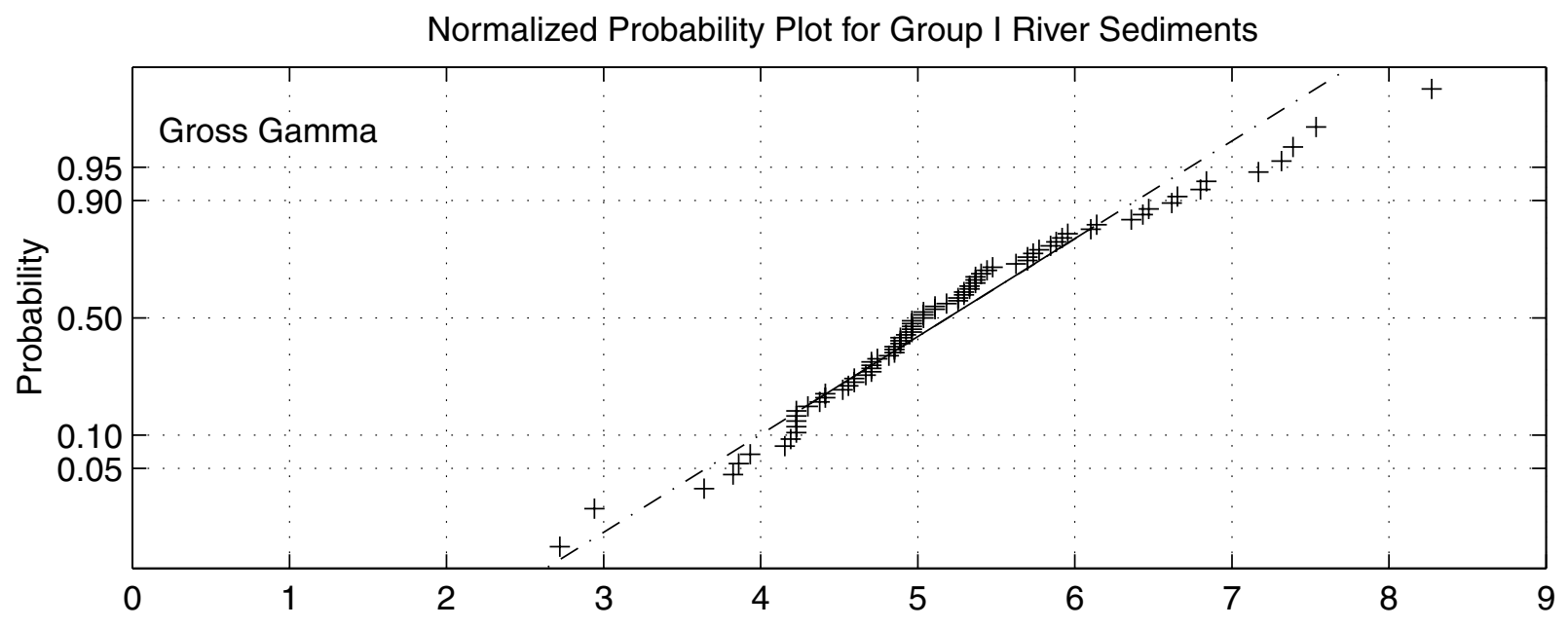

Natural Log Transform of Normalized Probability Plot

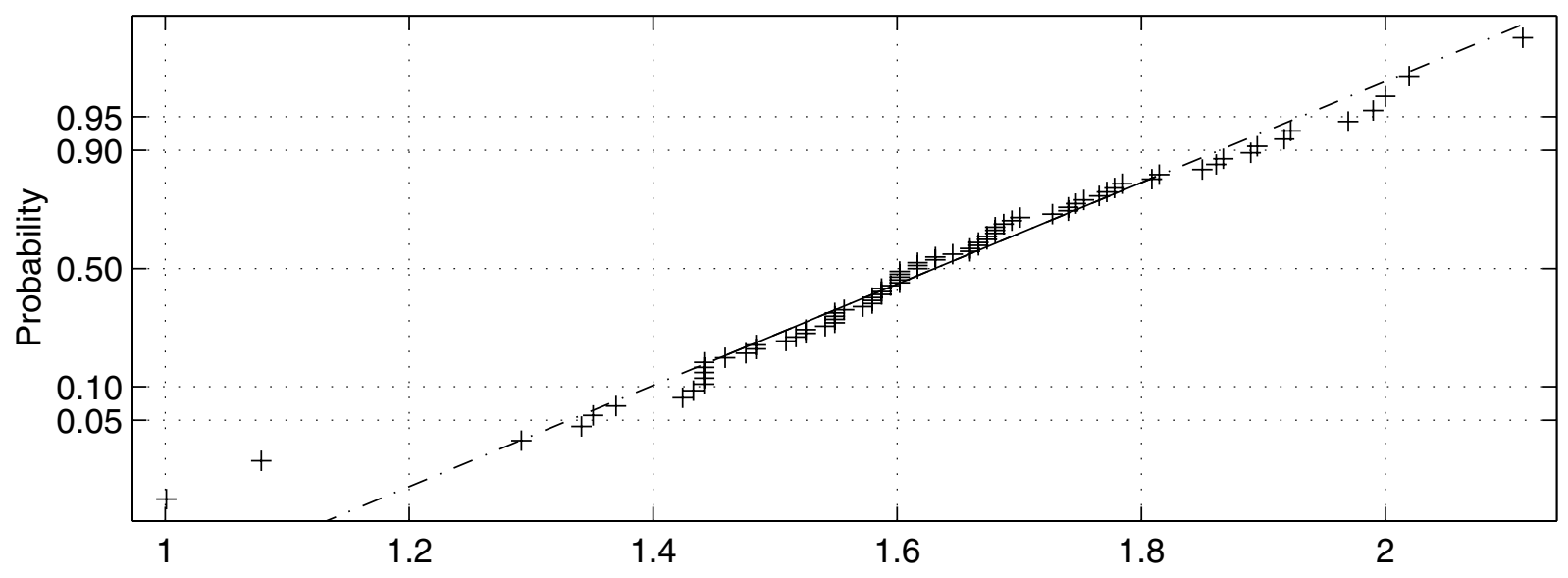

Square-Root Transform of Normalized Probability Plot

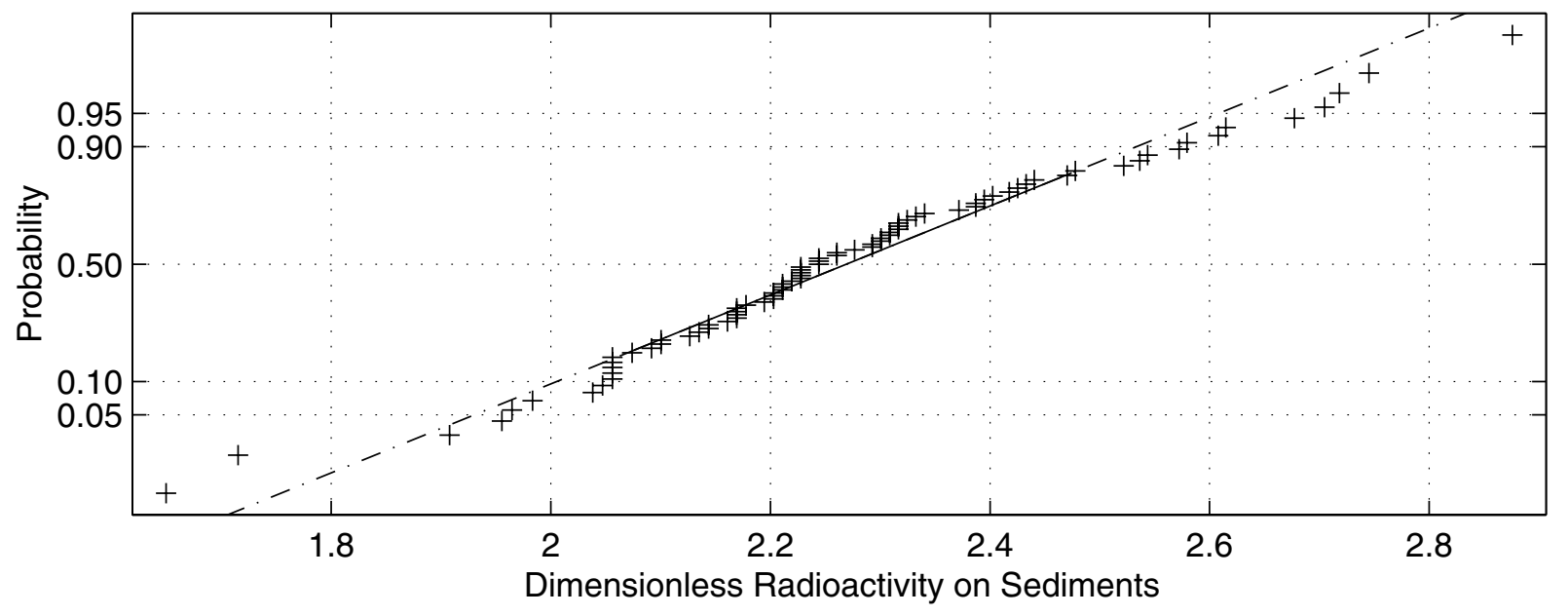

Fig. D-20. Normalized plots for gross gamma in Group I river sediments. 

APPENDIX E

PROBABILITY PLOTS FOR GROUP I RESERVOIR SEDIMENTS 


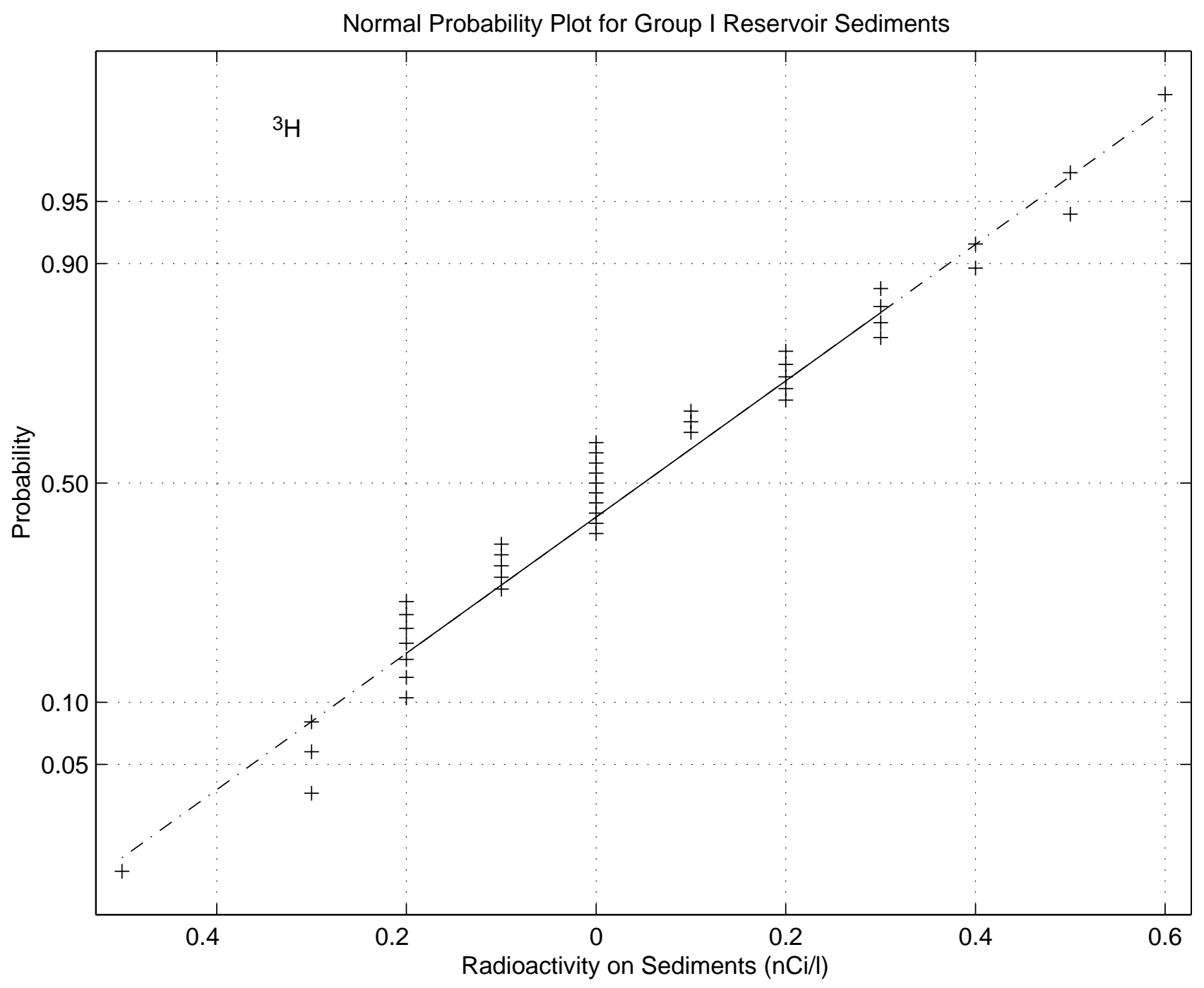

Fig. E-1. Probability plot for tritium in Group I reservoir sediments. 

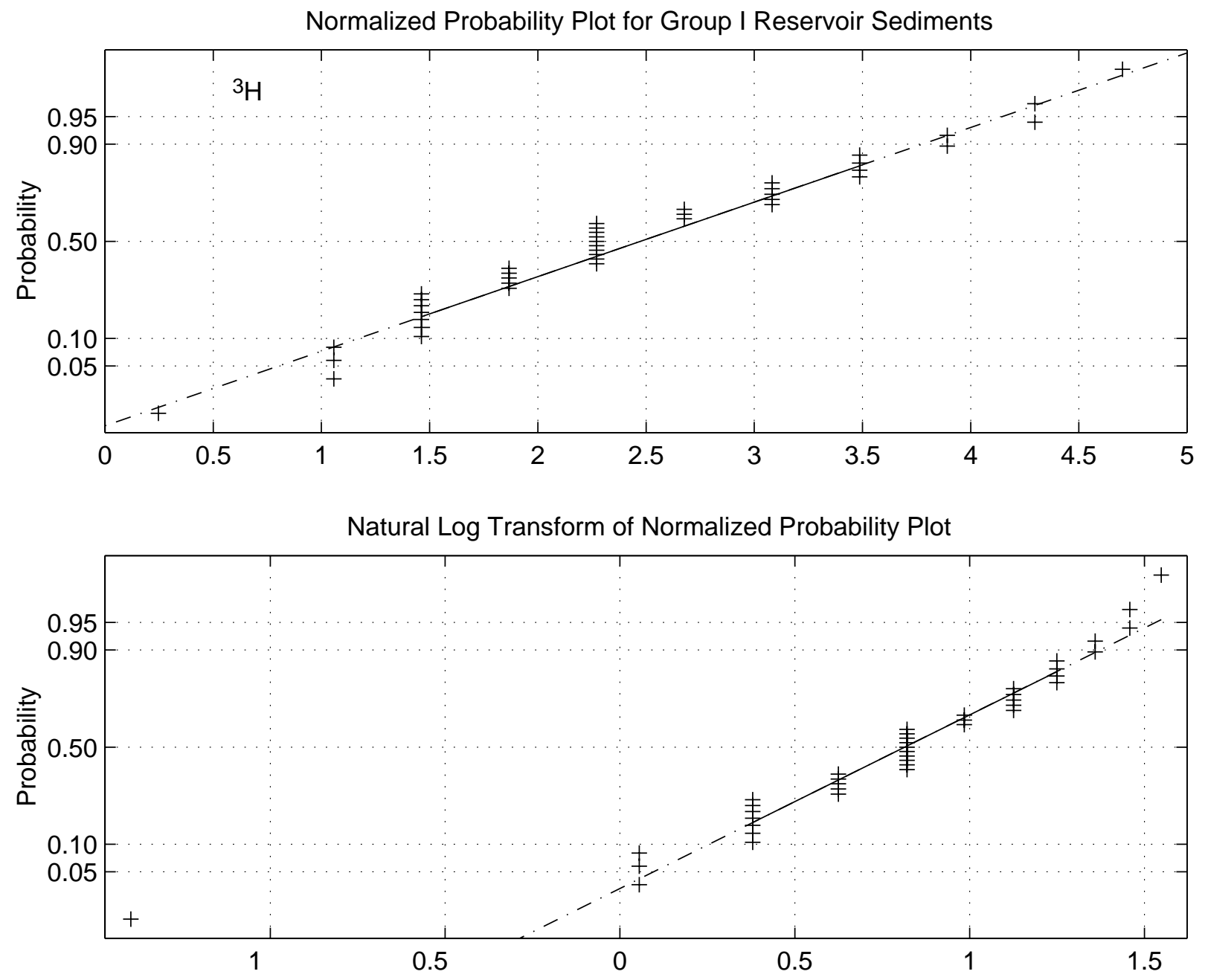

Square-Root Transform of Normalized Probability Plot

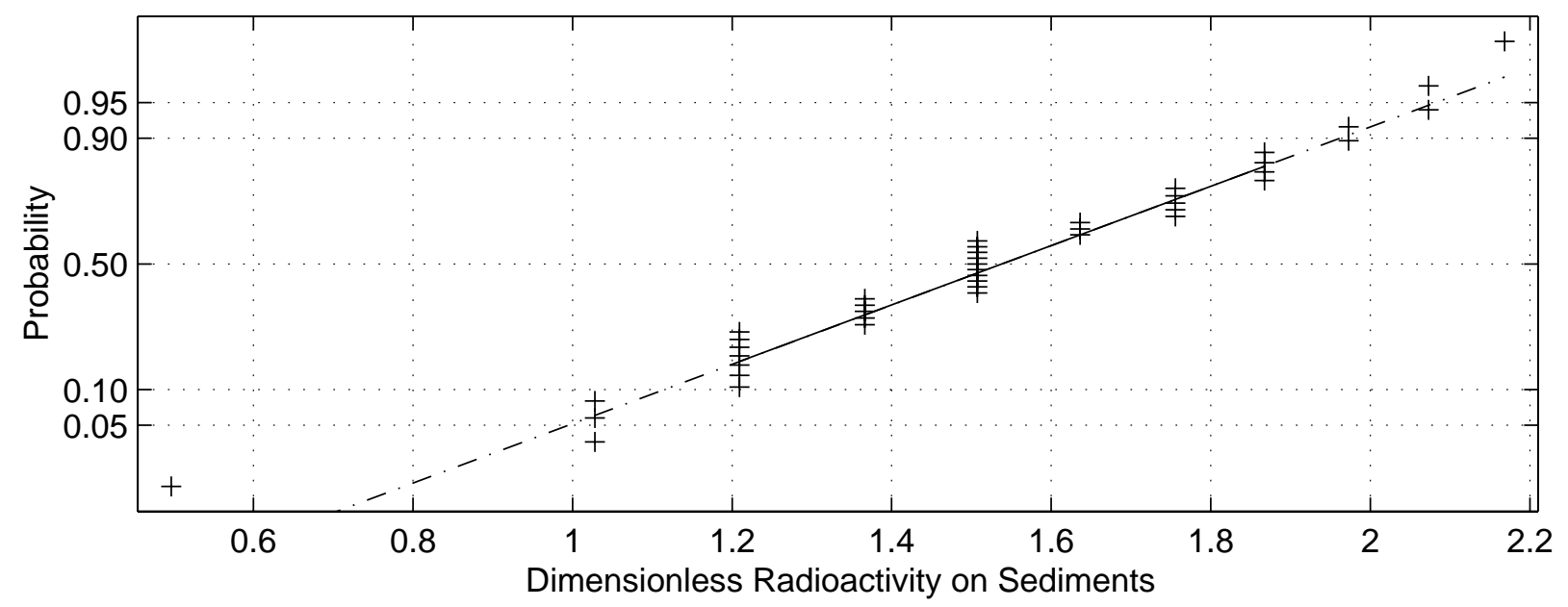

Fig. E-2. Normalized plots for tritium in Group I reservoir sediments. 


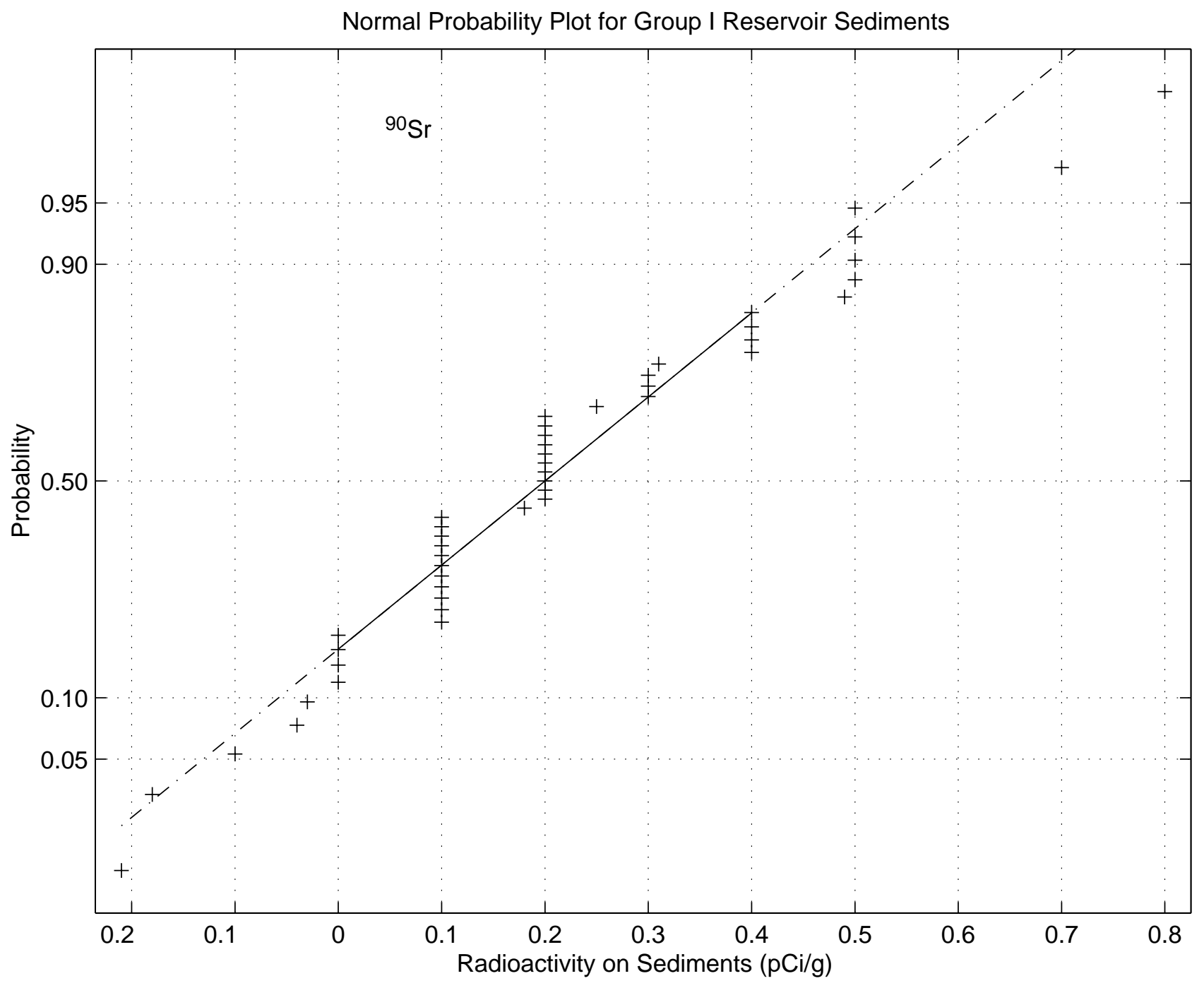

Fig. E-3. Probability plot for strontium-90 in Group I reservoir sediments. 

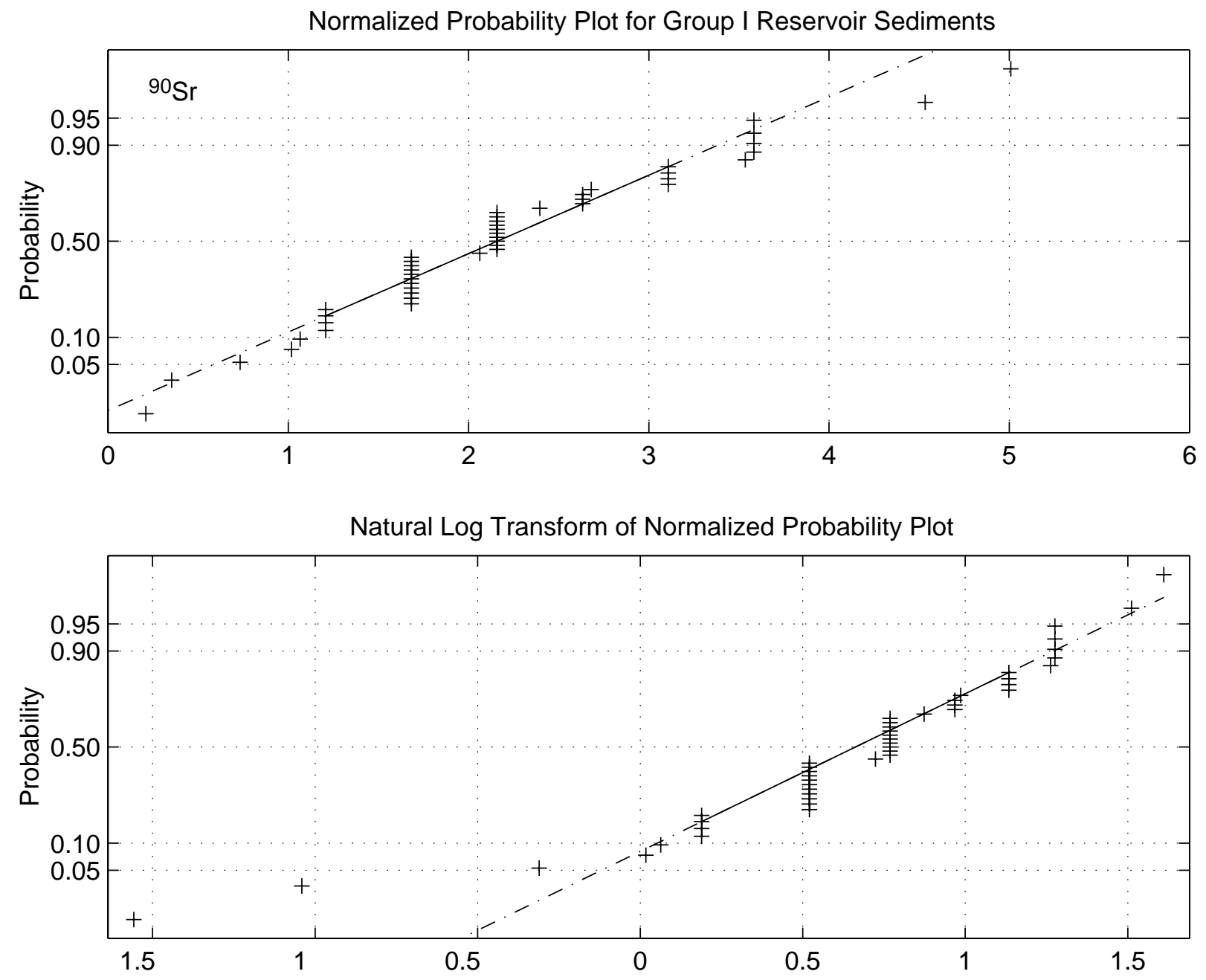

Square-Root Transform of Normalized Probability Plot

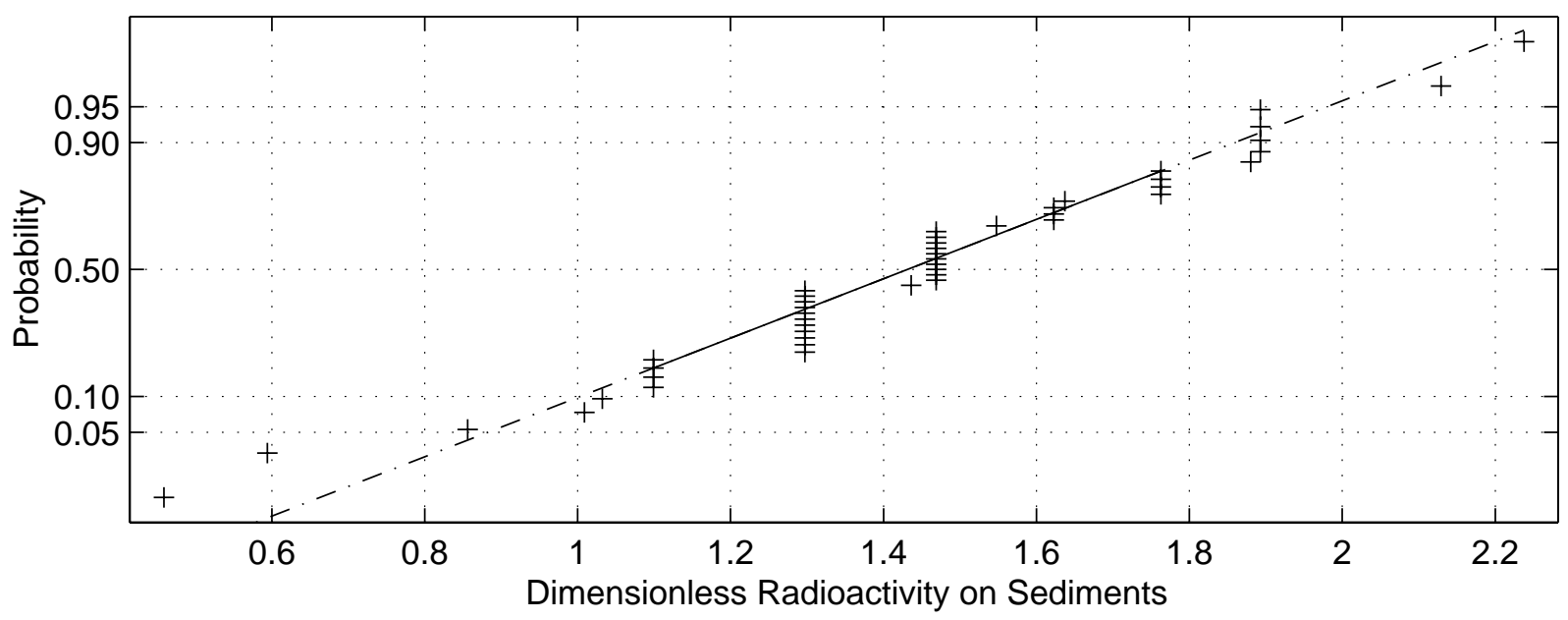

Fig. E-4. Normalized plots for strontium-90 in Group I reservoir sediments. 


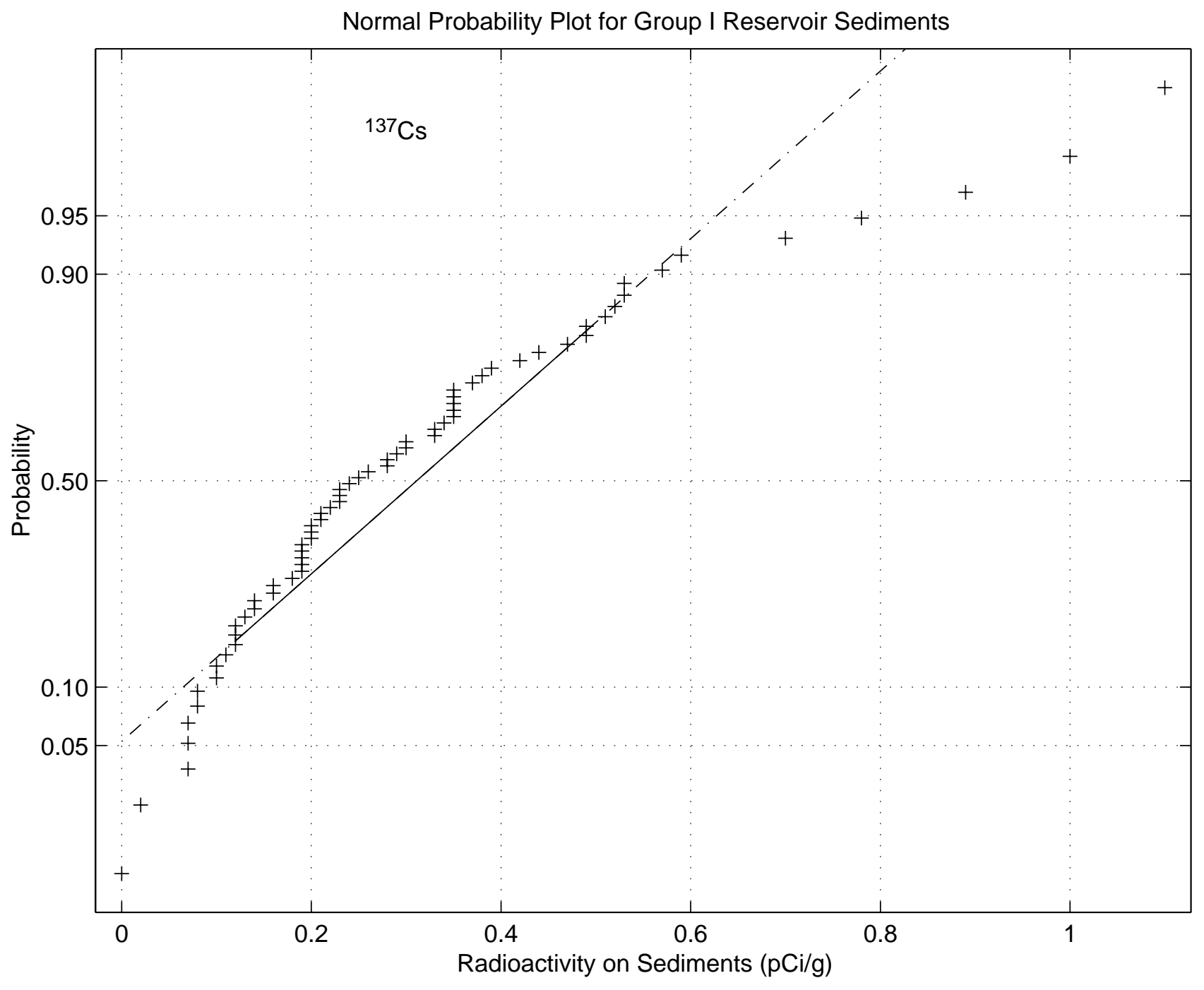

Fig. E-5. Probability plot for cesium-137 in Group I reservoir sediments. 

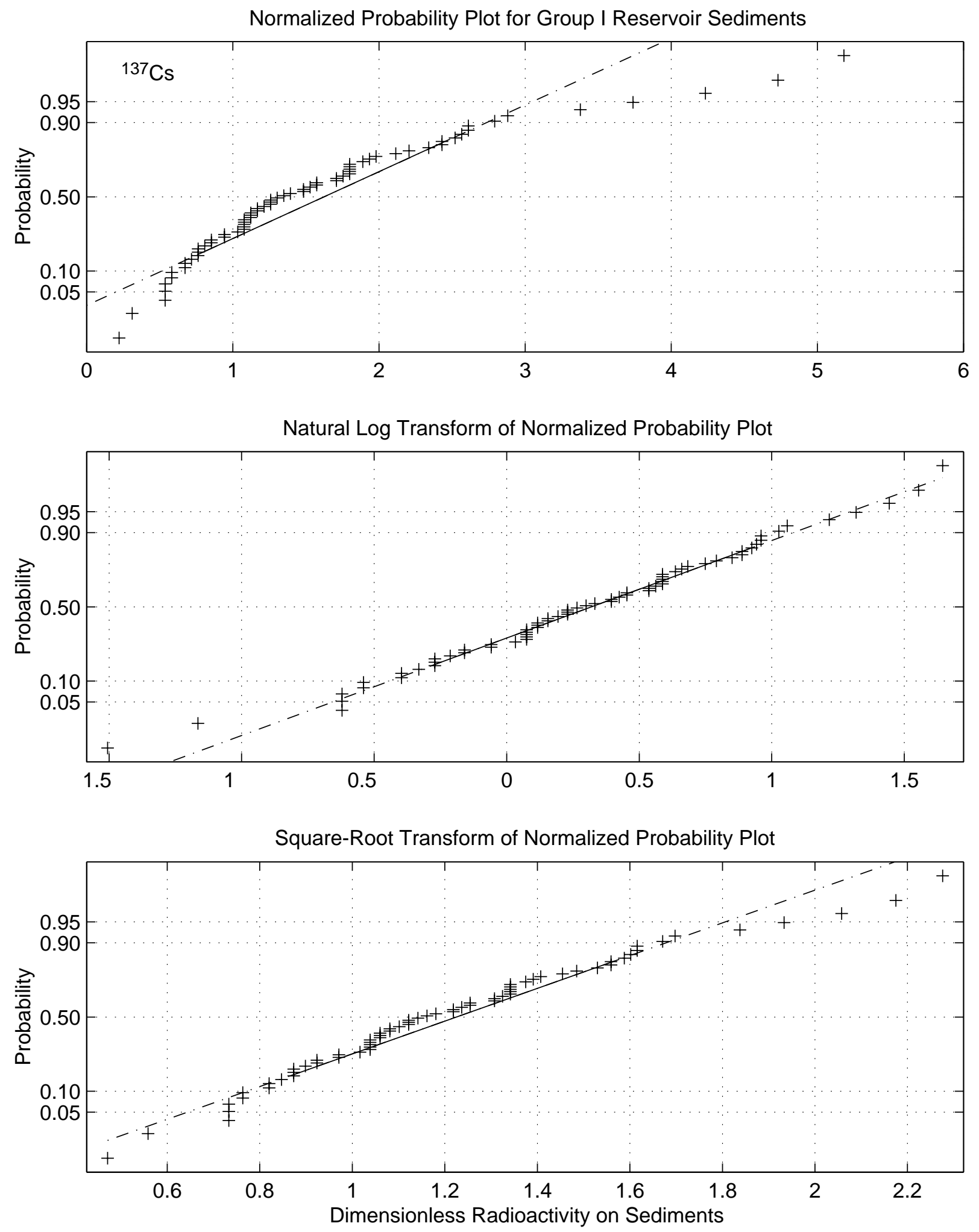

Fig. E-6. Normalized plots for cesium-137 in Group I reservoir sediments. 


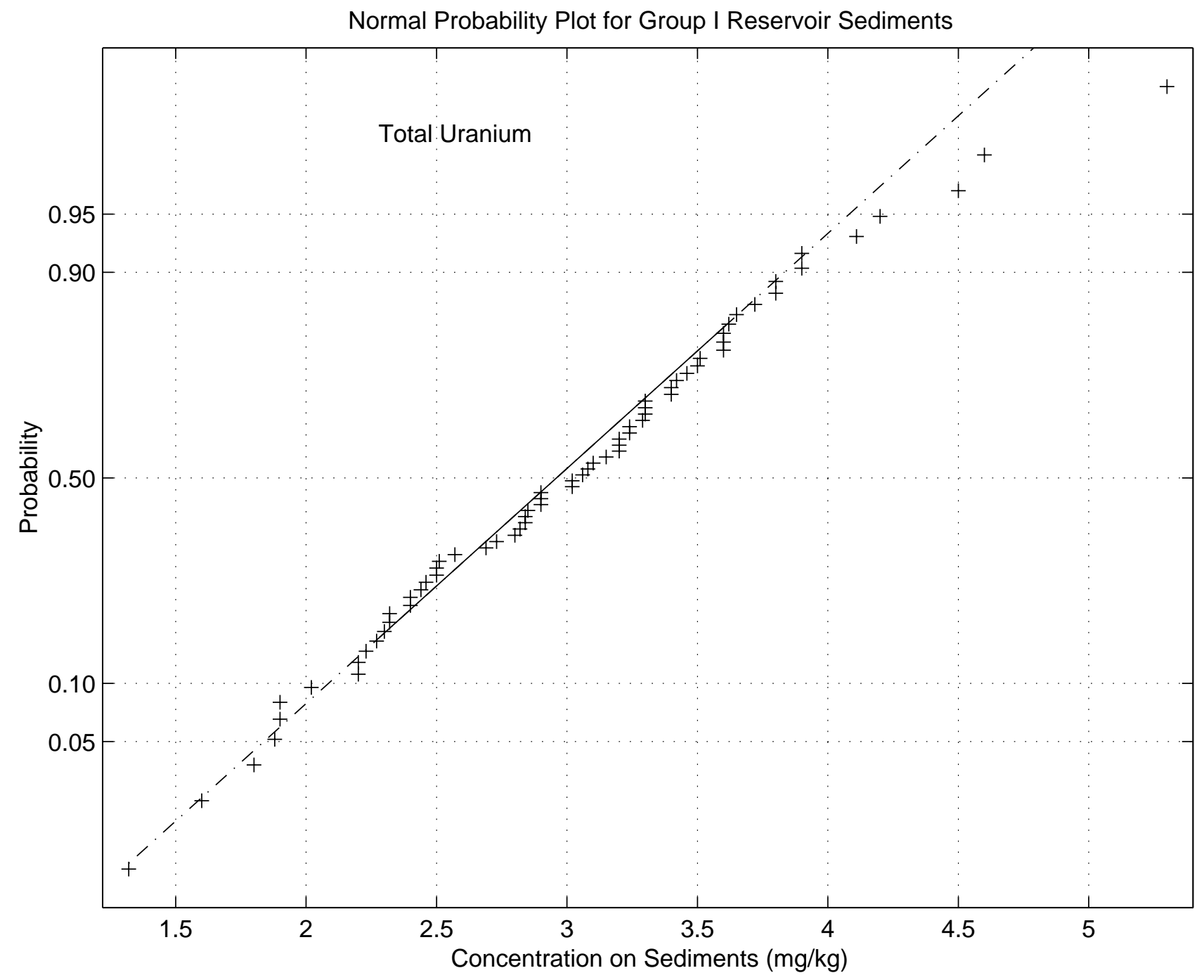

Fig. E-7. Probability plot for total uranium in Group I reservoir sediments. 

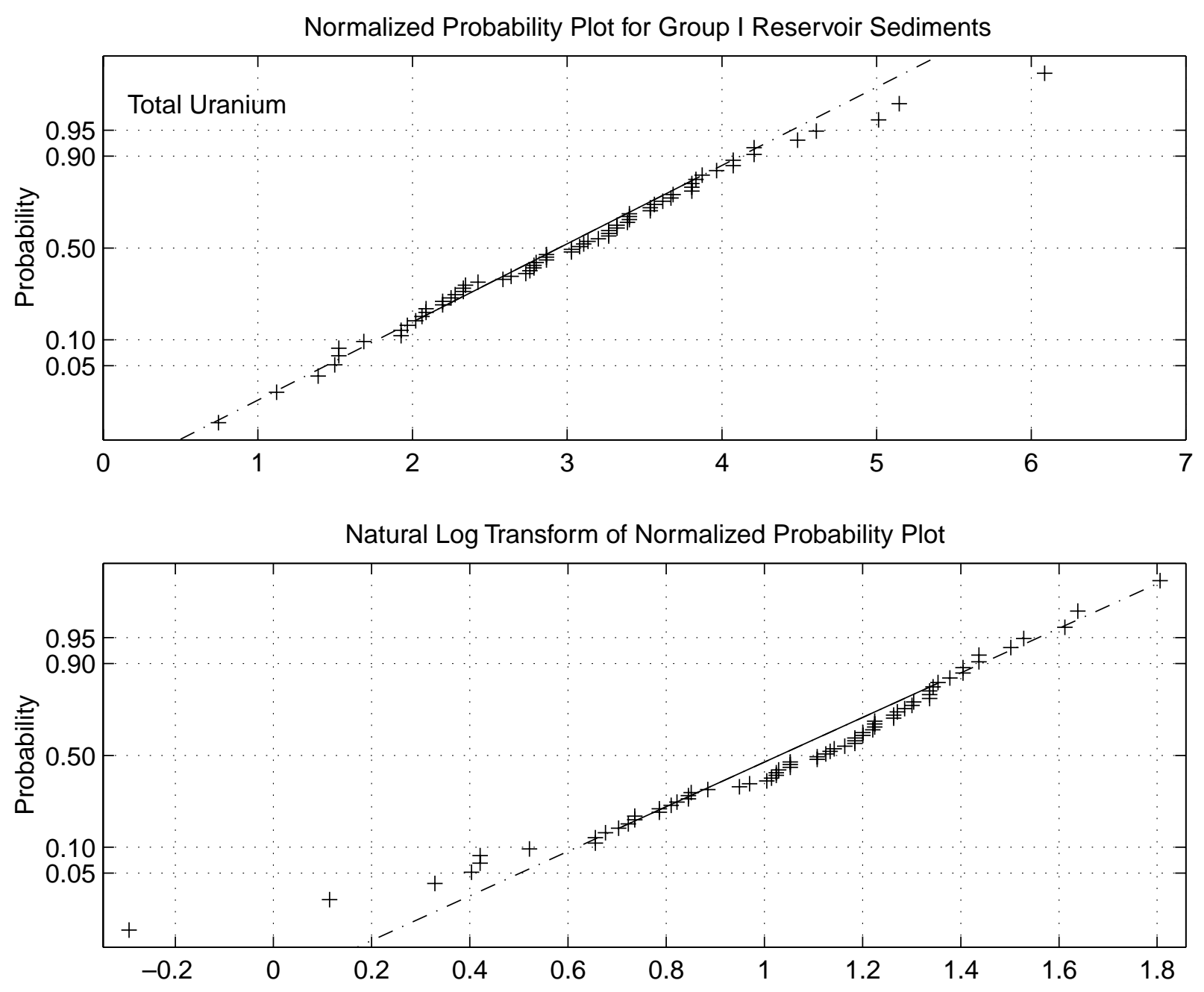

Square-Root Transform of Normalized Probability Plot

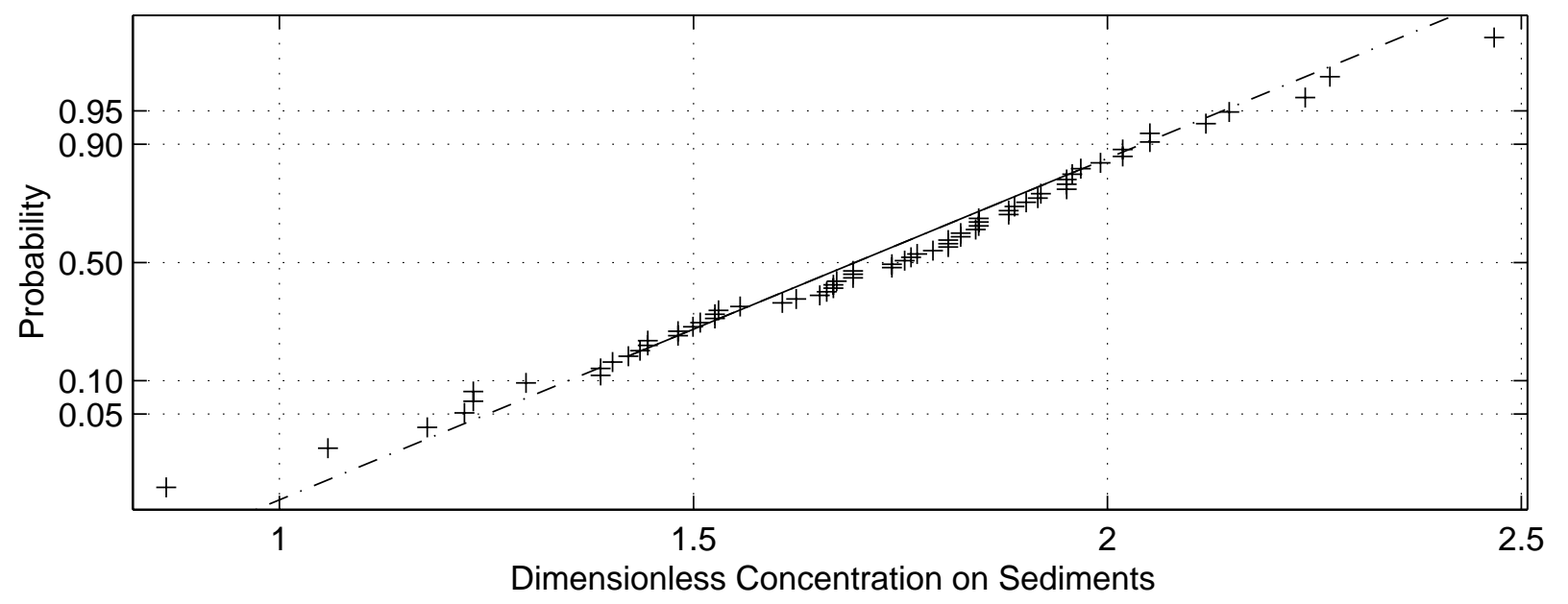

Fig. E-8. Normalized plots for total uranium in Group I reservoir sediments. 
Normal Probability Plot for Group I Reservoir Sediments

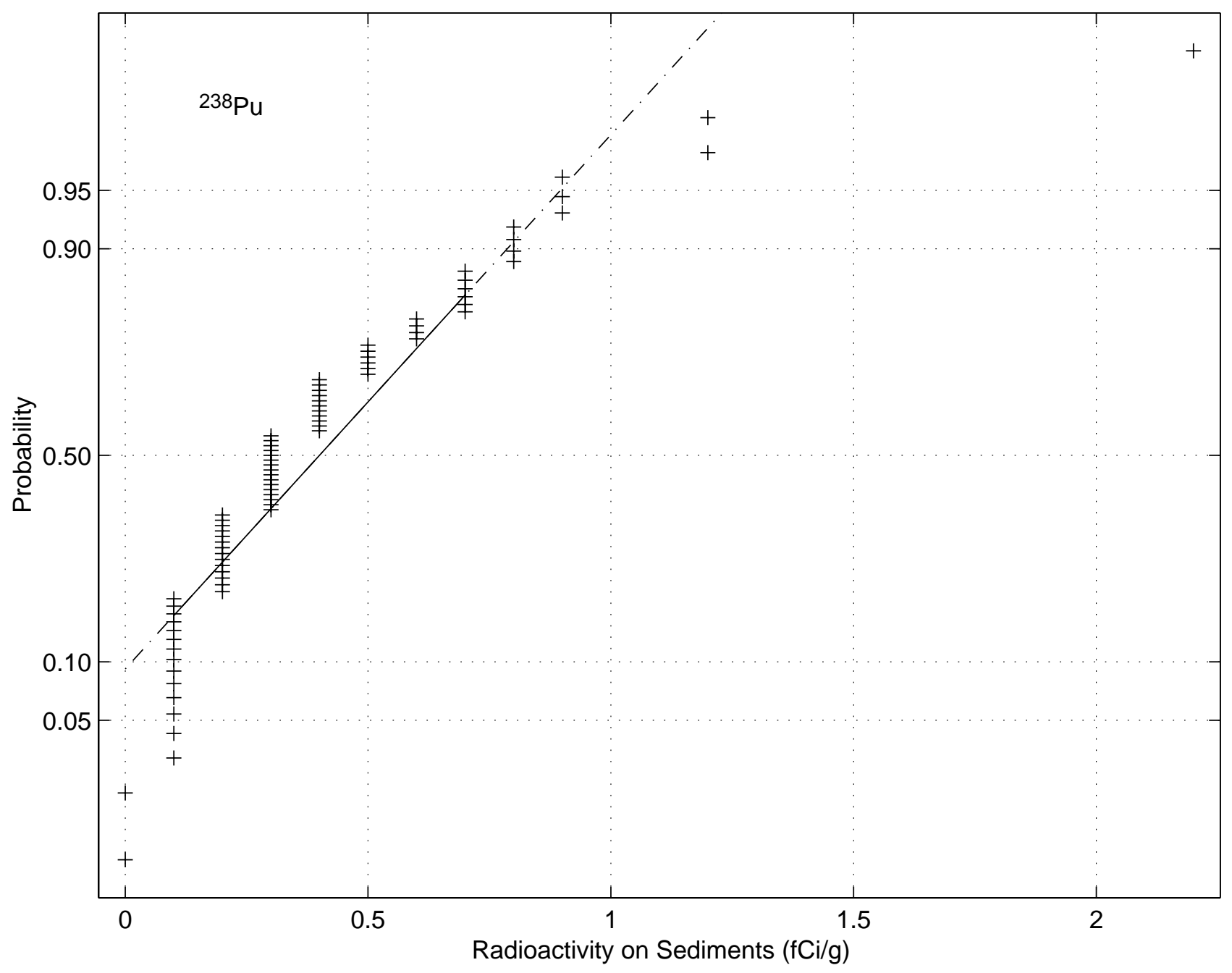

Fig. E-9. Probability plot for plutonium-238 in Group I reservoir sediments. 

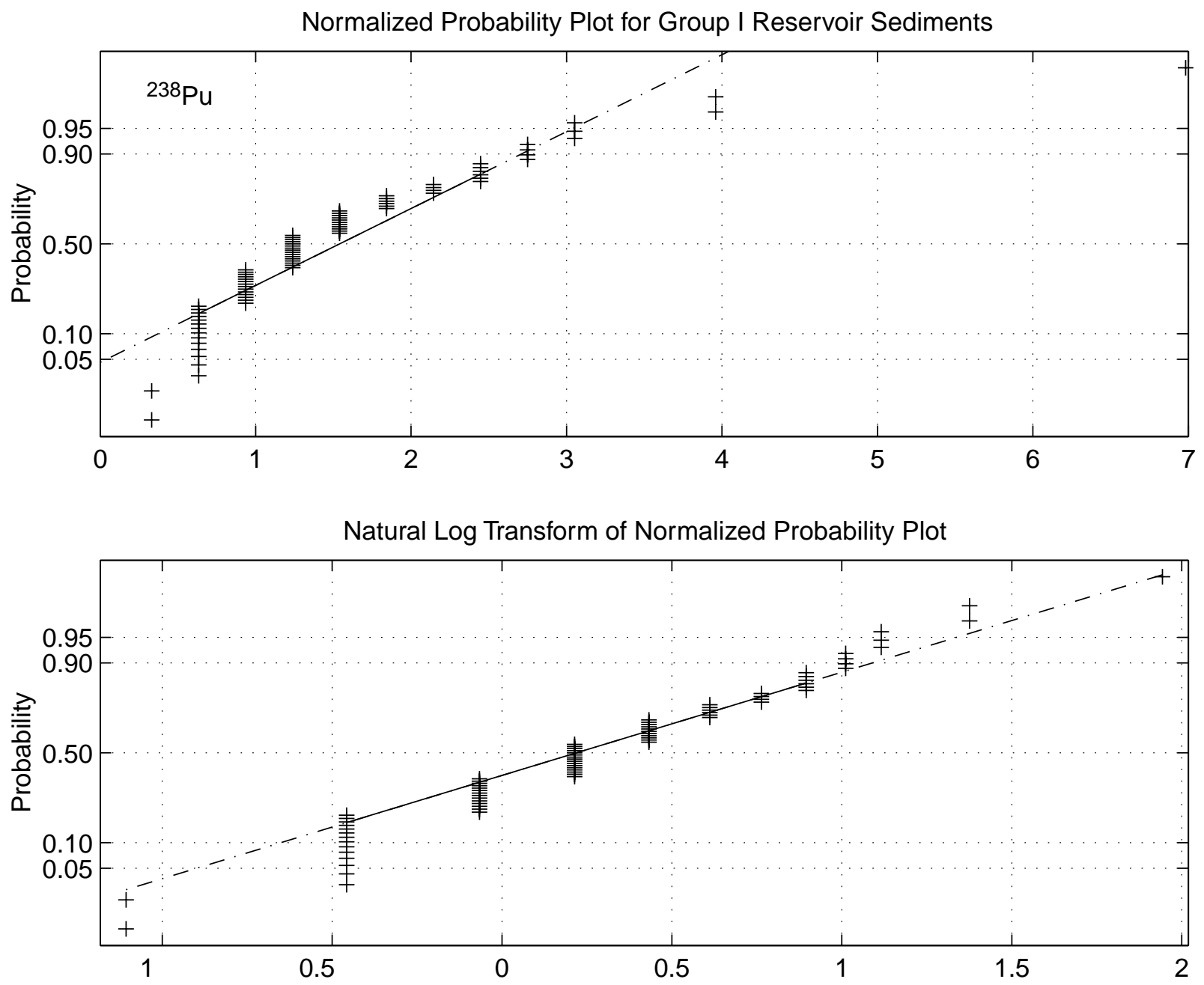

Square-Root Transform of Normalized Probability Plot

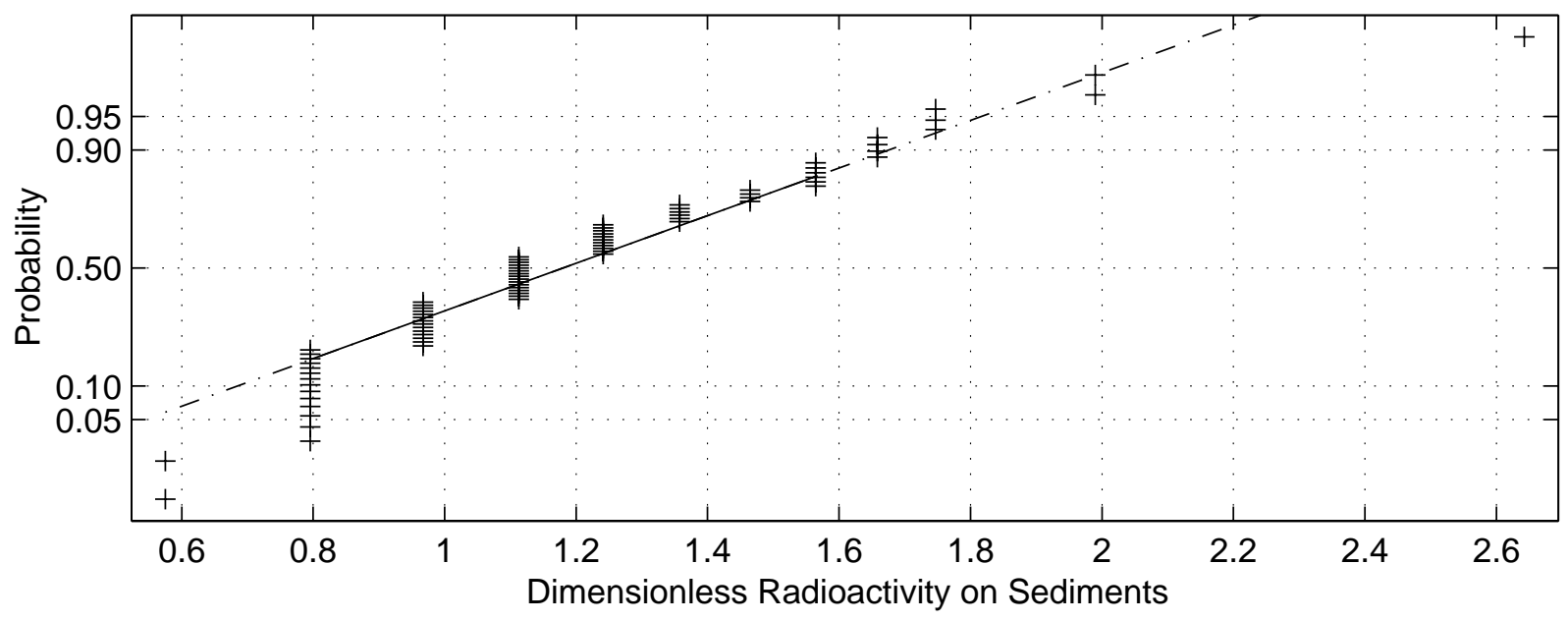

Fig. E-10. Normalized plots for plutonium-238 in Group I reservoir sediments. 


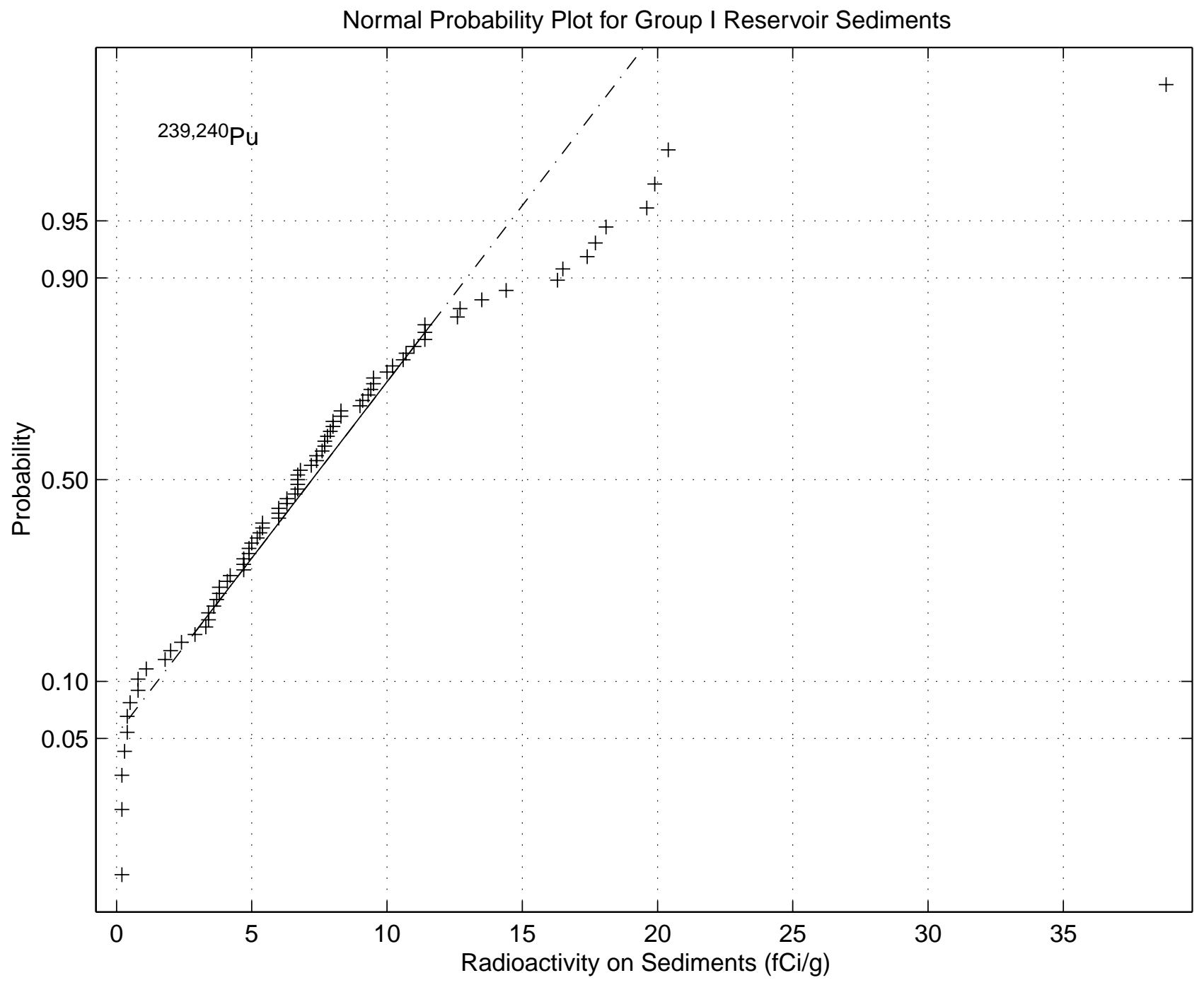

Fig. E-11. Probability plot for plutonium-239,-240 in Group I reservoir sediments. 

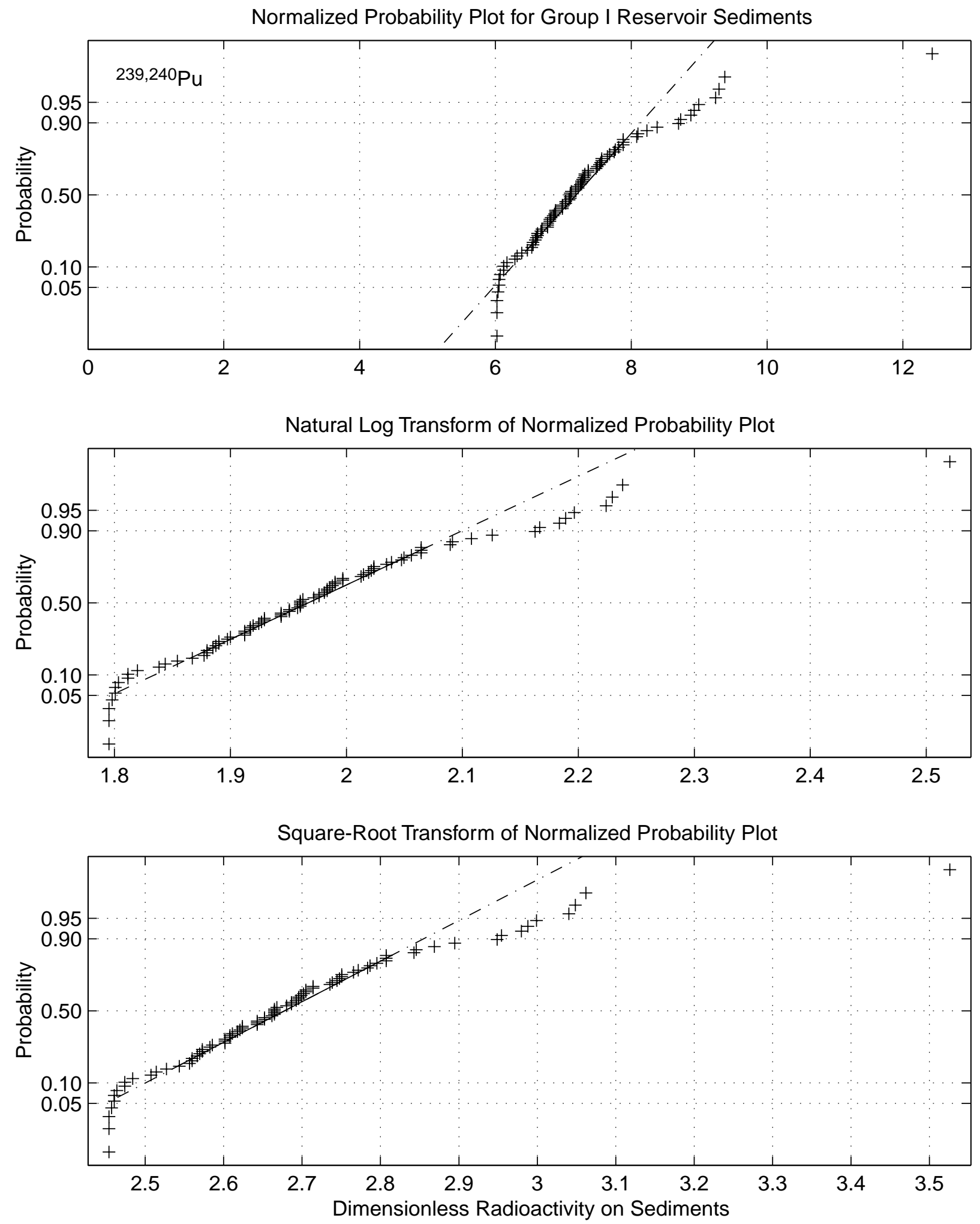

Fig. E-12. Normalized plots for plutonium-239,-240 in Group I reservoir sediments. 


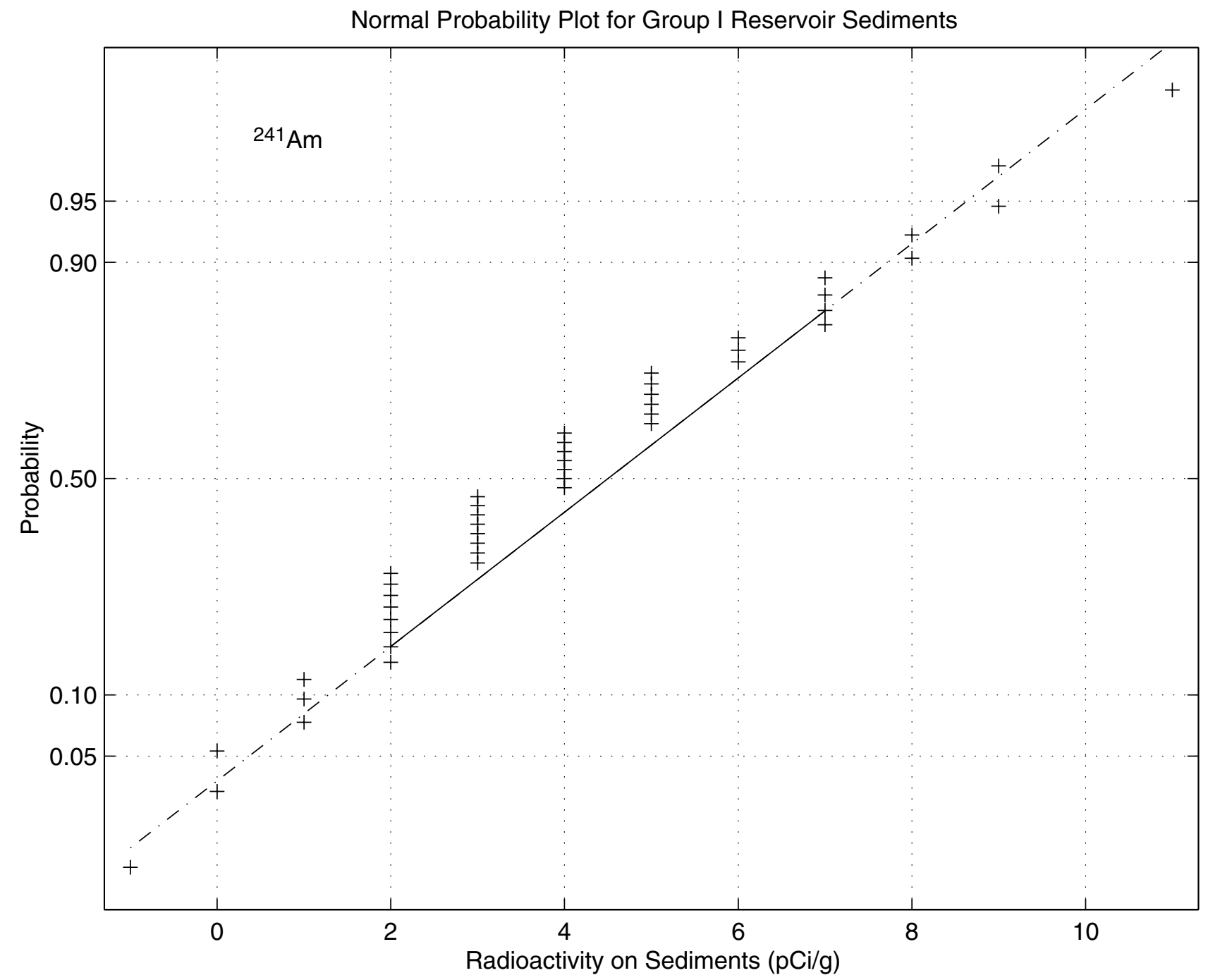

Fig. E-13. Probability plot for americium-241 in Group I reservoir sediments. 

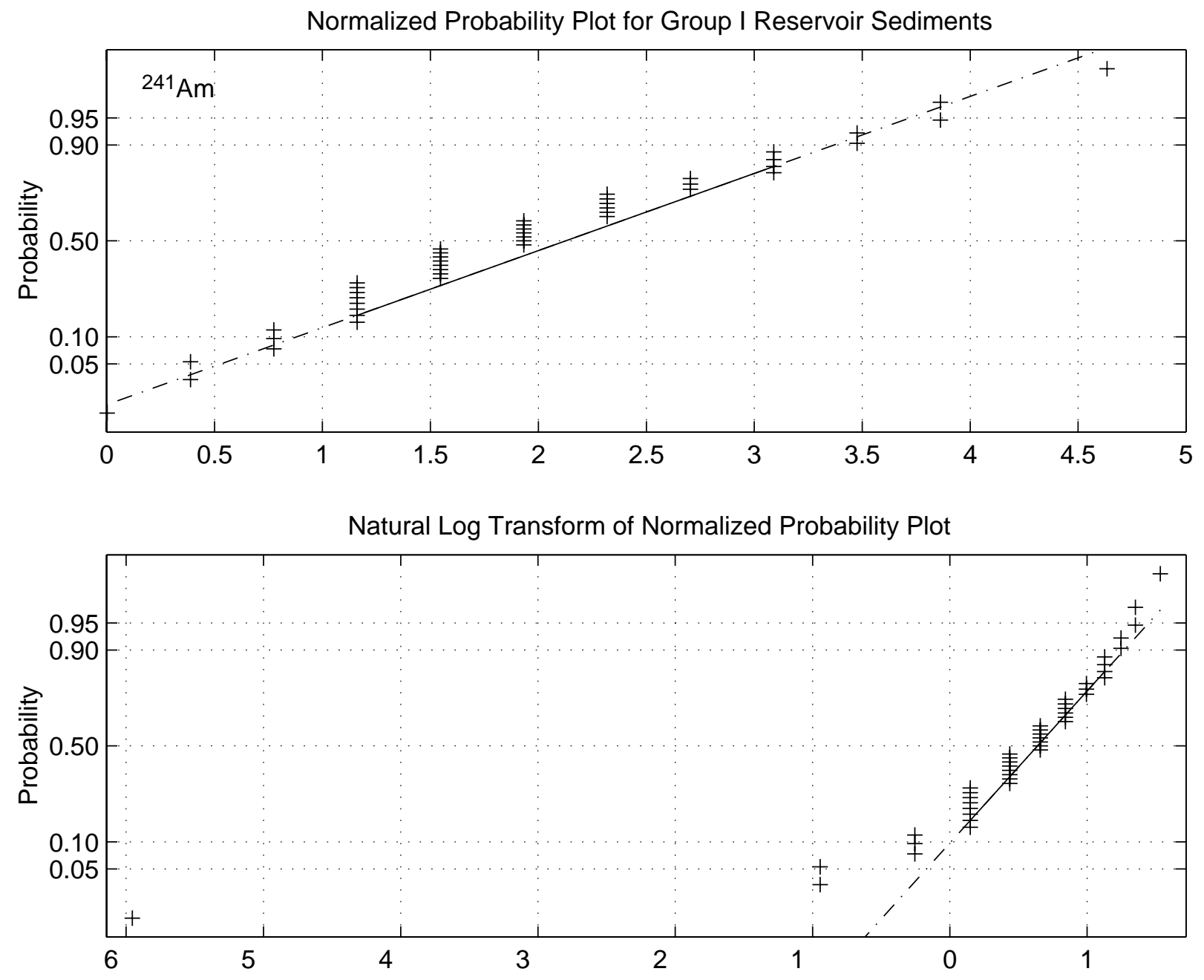

Square-Root Transform of Normalized Probability Plot

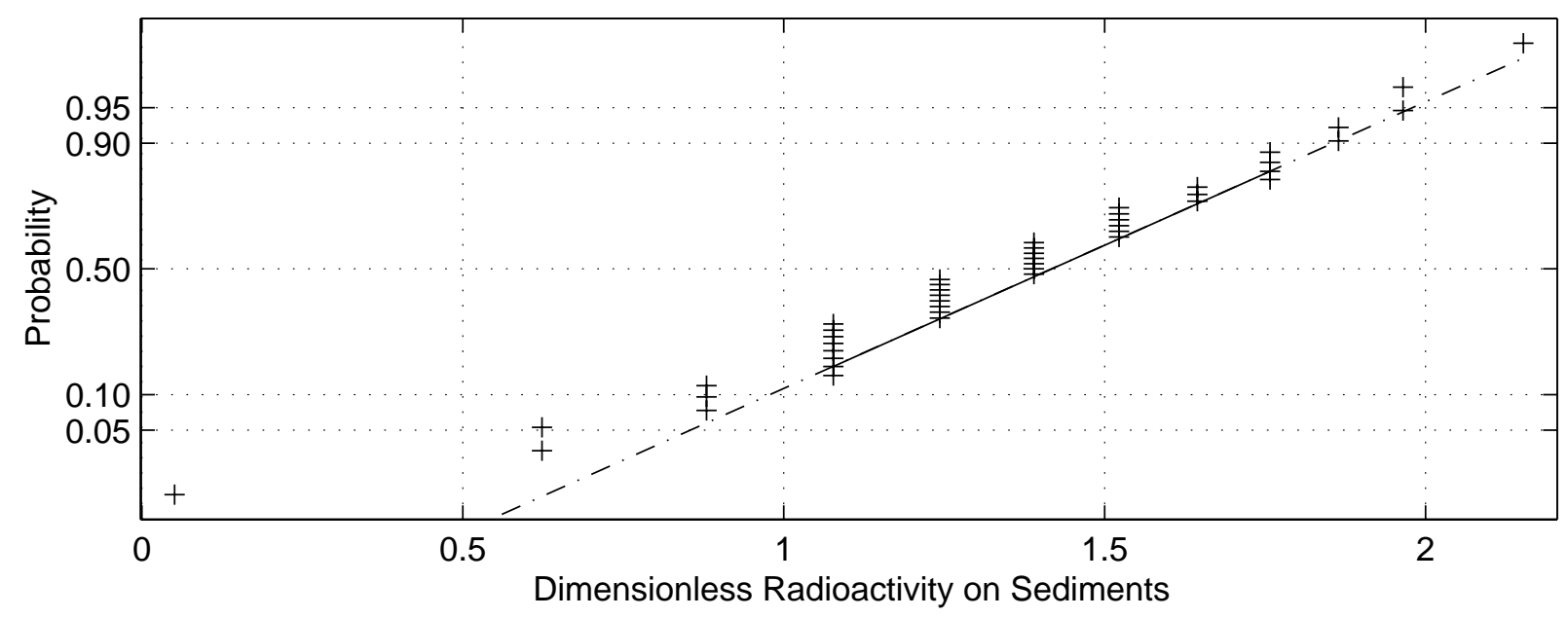

Fig. E-14. Normalized plots for americium-241 in Group I reservoir sediments. 


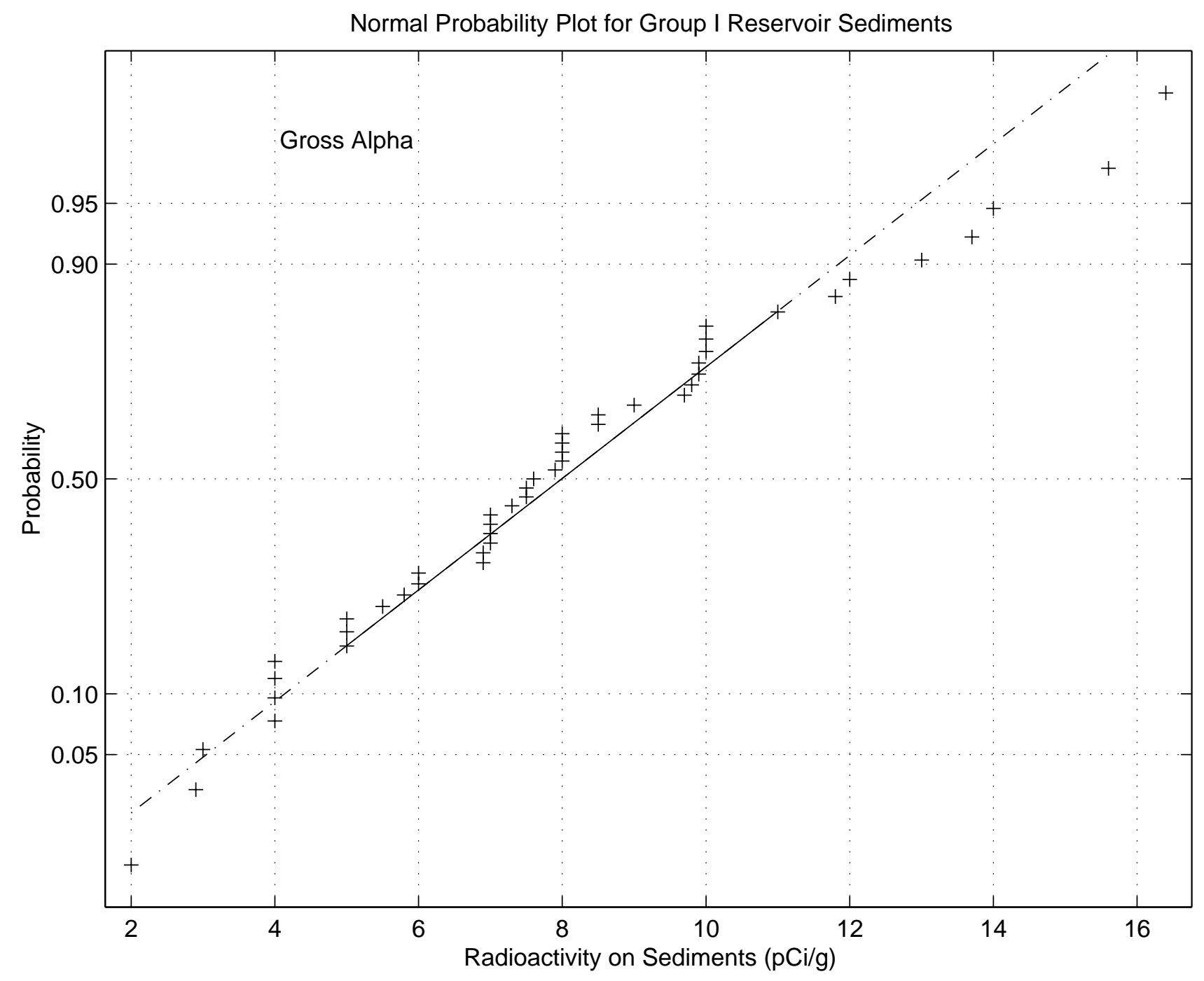

Fig. E-15. Probability plot for gross alpha in Group I reservoir sediments. 
Normalized Probability Plot for Group I Reservoir Sediments

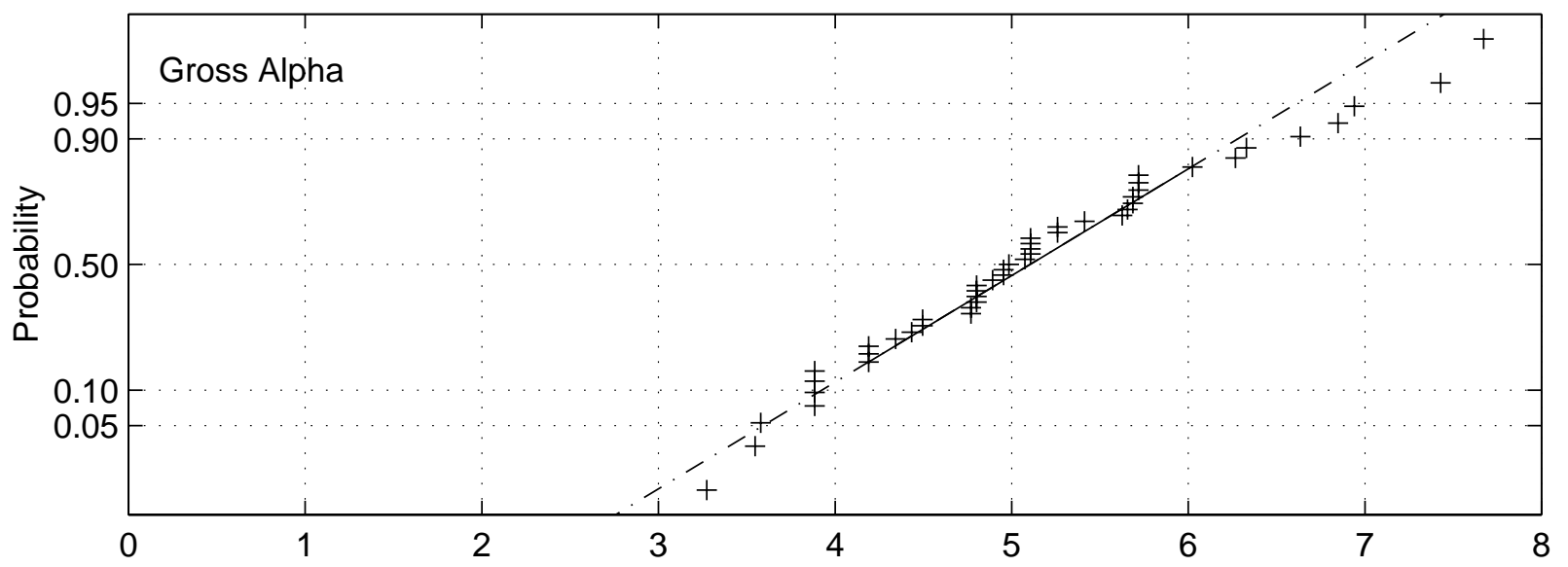

Natural Log Transform of Normalized Probability Plot

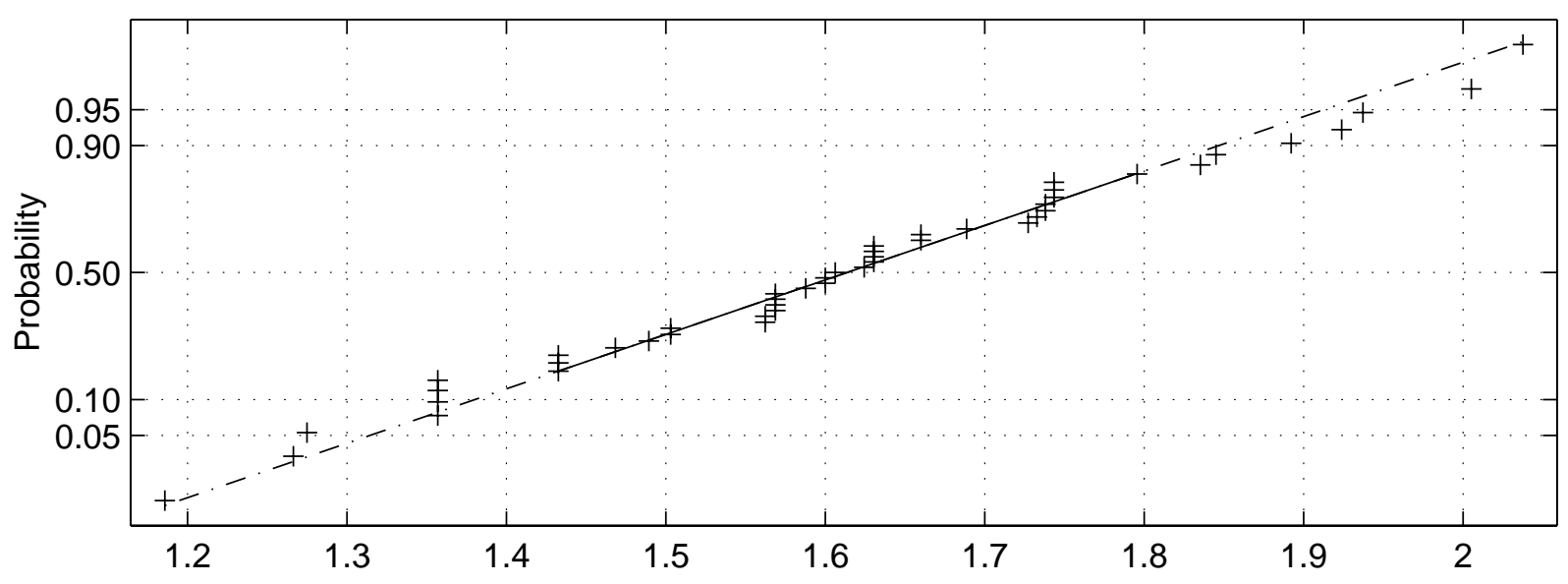

Square-Root Transform of Normalized Probability Plot

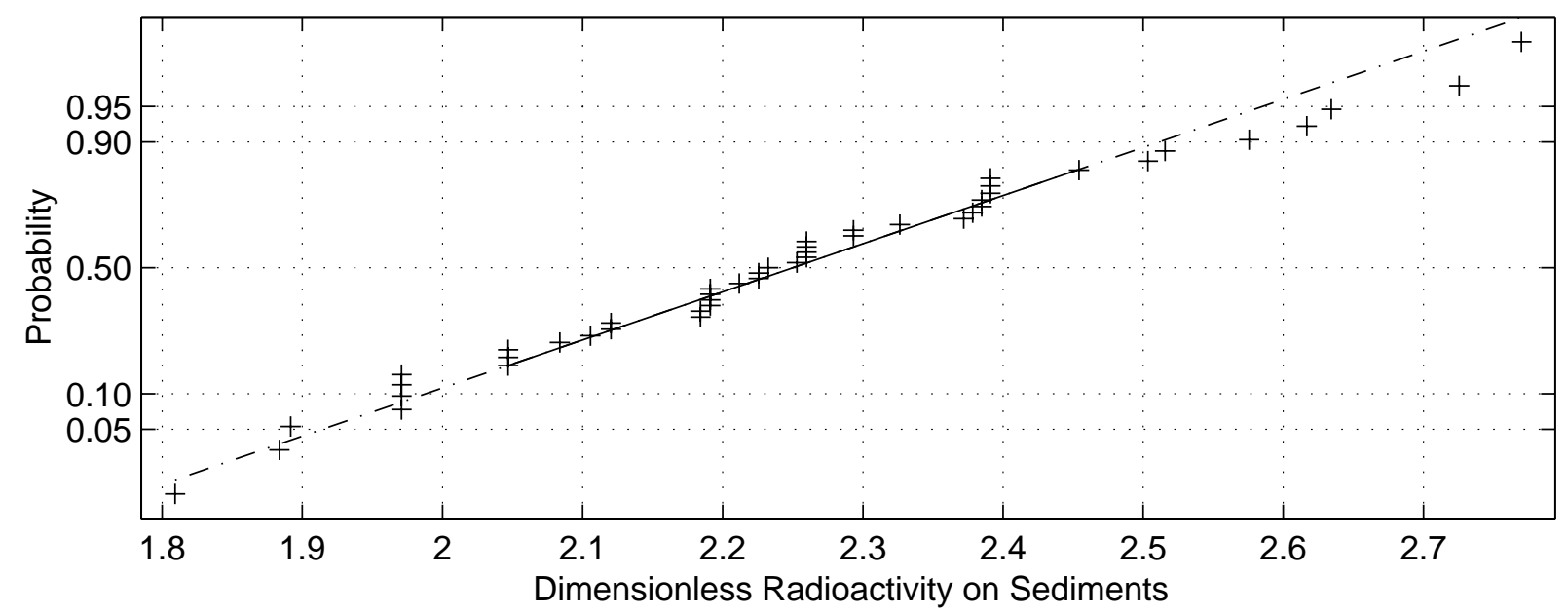

Fig. E-16. Normalized plots for gross alpha in Group I reservoir sediments. 


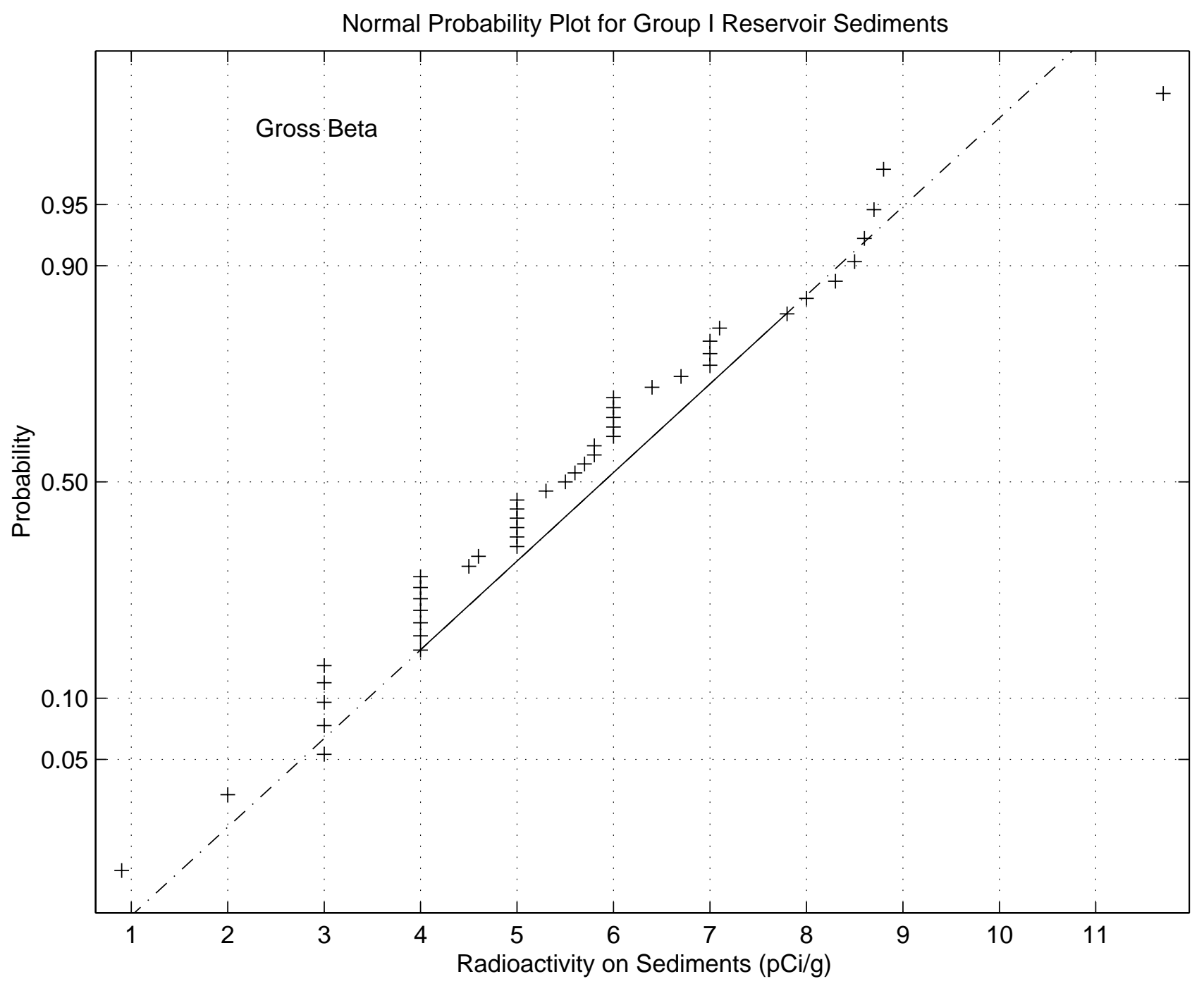

Fig. E-17. Probability plot for gross beta in Group I reservoir sediments. 

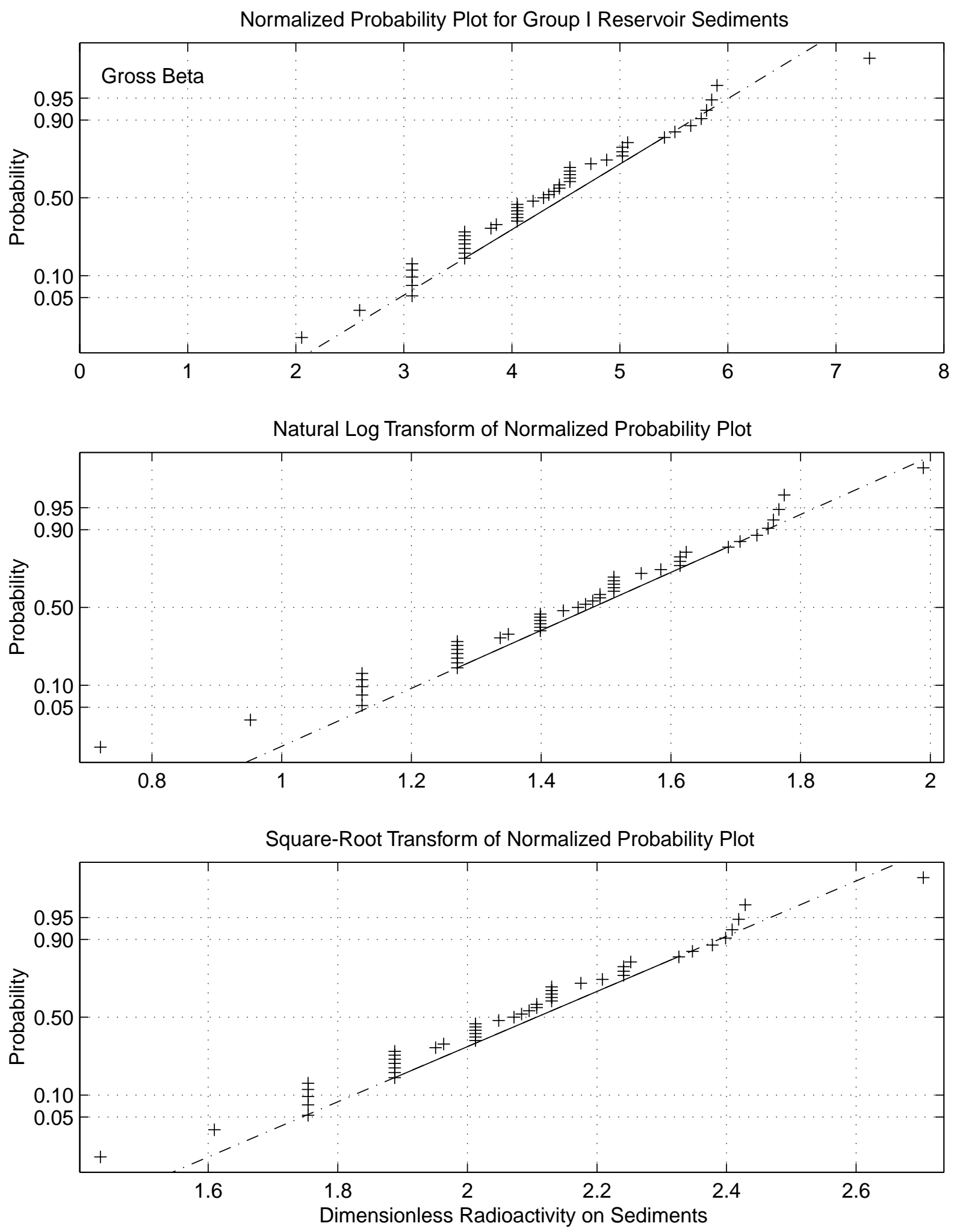

Fig. E-18. Normalized plots for gross beta in Group I reservoir sediments. 


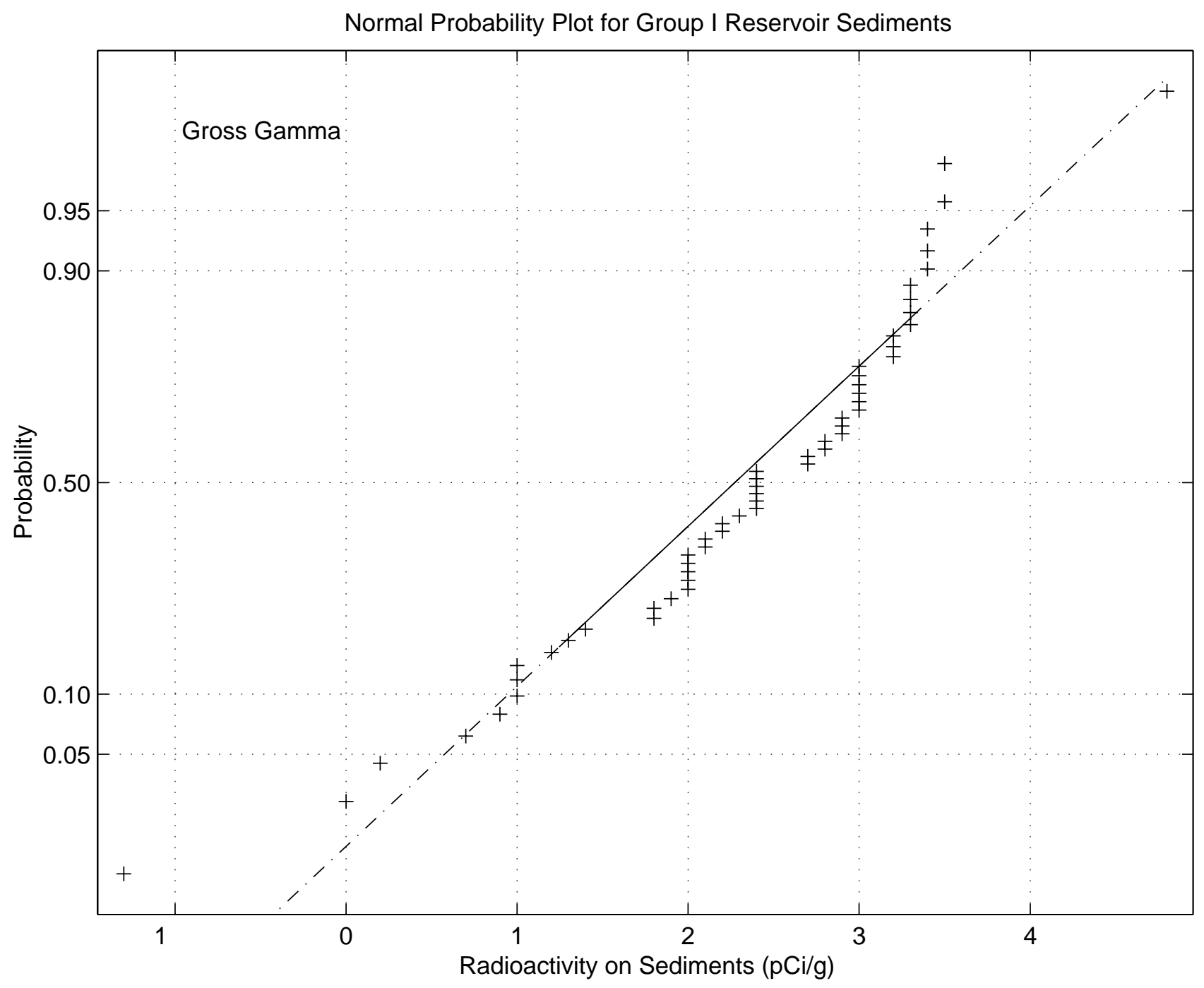

Fig. E-19. Probability plot for gross gamma in Group I reservoir sediments. 

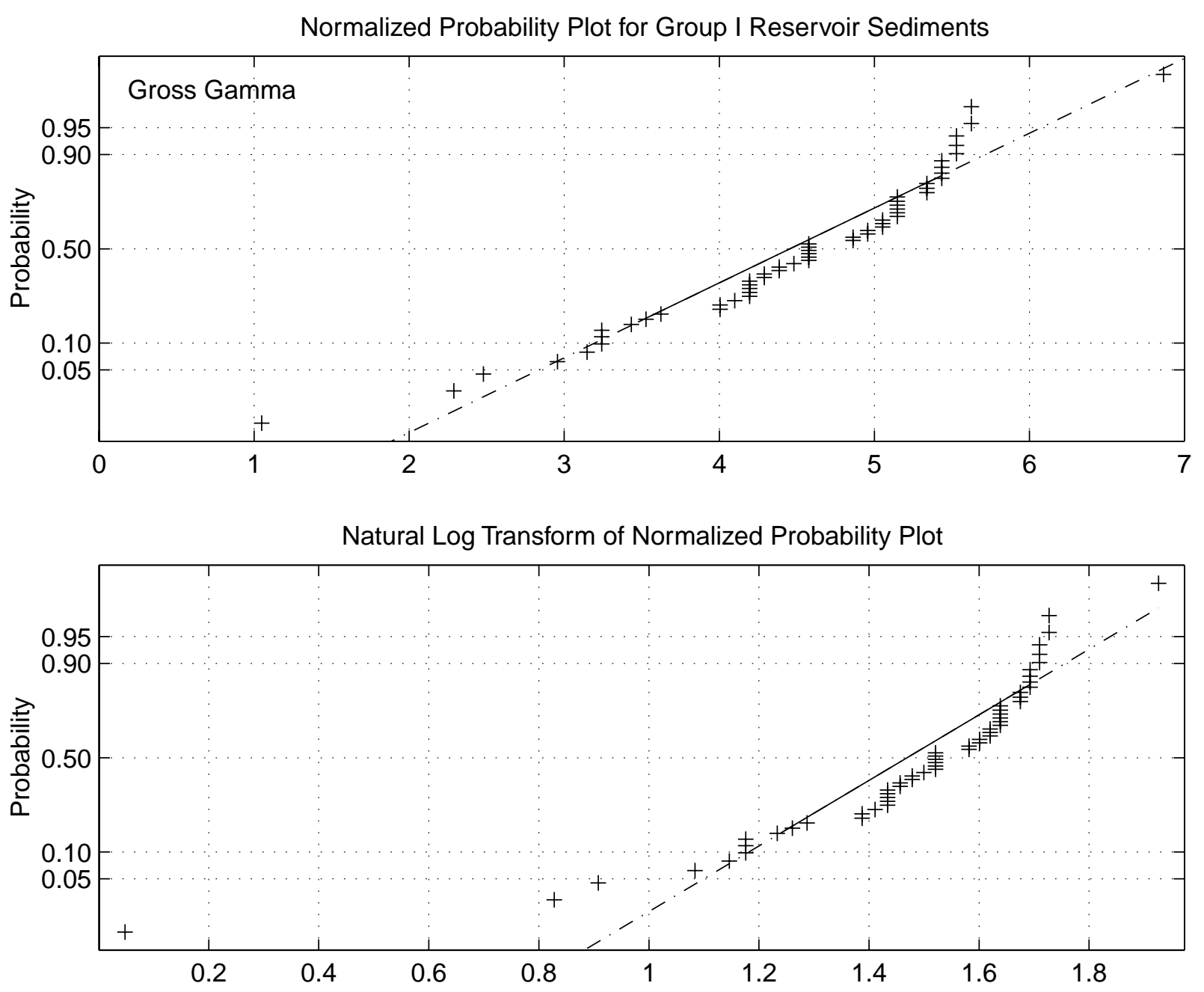

Square-Root Transform of Normalized Probability Plot

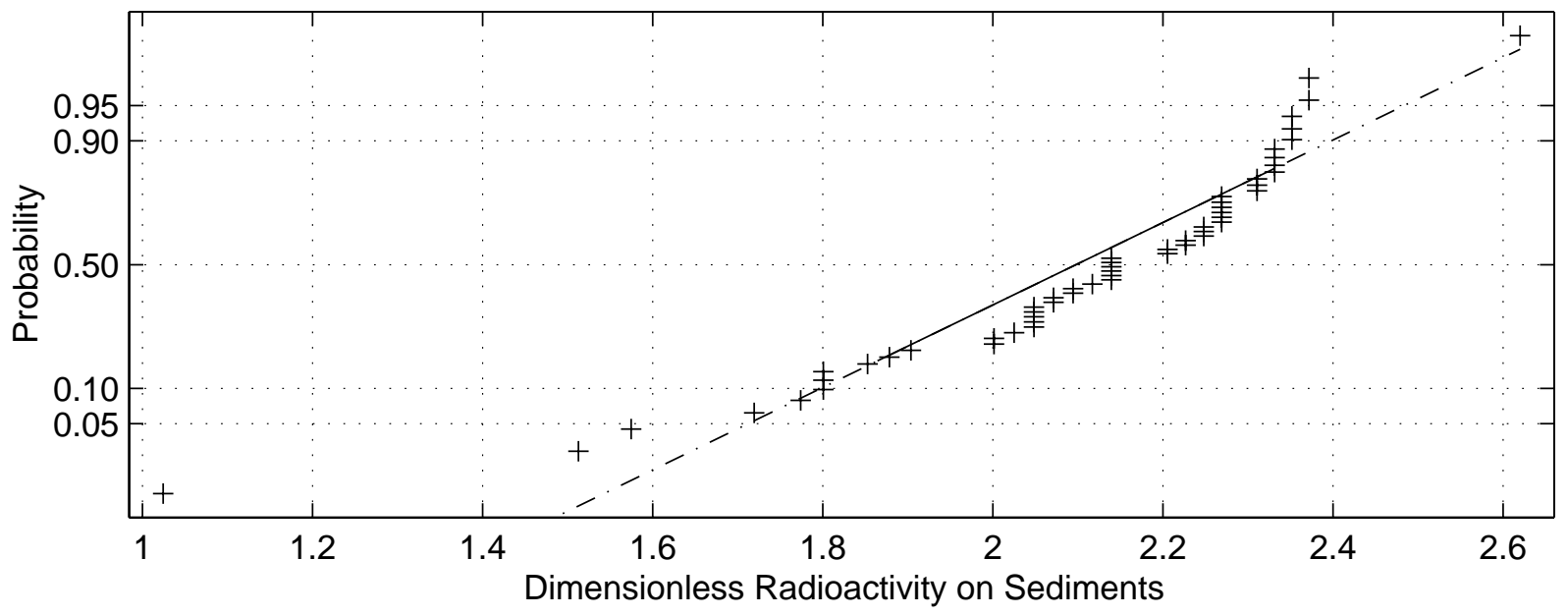

Fig. E-20. Normalized plots for gross gamma in Group I reservoir sediments. 

This report has been reproduced directly from the best available copy. It is available electronically on the Web (http://www.doe.gov/bridge).

Copies are available for sale to U.S. Department of Energy employees and contractors from-

Office of Scientific and Technical Information

P.O. Box 62

Oak Ridge, TN 37831

(865) 576-8401

Copies are available for sale to the public from-

National Technical Information Service

US Department of Commerce

5285 Port Royal Road

Springfield, VA 22616

(800) 553-6847 


\section{Los Alamos \\ NATIONAL LABORATORY \\ Los Alamos, New Mexico 87545}

